\title{
The Role of the Serotonin Transporter as a Genetic Risk Factor in the Development of Drug Addiction
}

by

Bridget Williams Brox

\author{
A thesis \\ submitted to the Victoria University of Wellington \\ in fulfilment of the \\ requirements for the degree of \\ Doctor of Philosophy \\ in Psychology.
}

Victoria University of Wellington 2016 



\begin{abstract}
Drug addiction is a ubiquitous phenomenon worldwide that places tremendous financial and psychological burden on societies, families and the individual. Interestingly, only a small percentage of individuals $(\sim 20 \%)$, regardless their drug of choice, go on to develop the compulsive behaviours that define drug addiction. Clinical studies have shown that there is a subset of the population with a genetically determined reduction in the serotonin transporter that may increase vulnerability to developing a variety of psychiatric disorders like depression, anxiety and drug addiction.

To investigate the influence of reduced serotonin transporter function in the laboratory we studied the effects of MDMA ('ecstasy') and heroin in a genetically altered animal model: the serotonin transporter (SERT) knockout rat. Homozygous (HOM) animals lack SERT function completely while heterozygous (HET) have about 50\% SERT function compared to the wild type (WT). Groups of HOM, HET and WT animals completed MDMA or heroin self-administration experiments. A robust genotype effect emerged for animals self-administering MDMA; facilitation of MDMA self-administration was inversely related to SERT function. HOM animals, without exception, reached acquisition criterion significantly faster than the HET animals; HET animals then showed higher acquisition rates compared to the WT animals. In contrast, there were no differences between the genotypes when animals self-administered heroin. To investigate the driving force behind facilitated MDMA self-administration in animals with reduced SERT function locomotor activity and conditioned taste aversion experiments were undertaken. In contrast to the drug selfadministration experiments, MDMA induced hyperactivity was positively
\end{abstract}


related to SERT function. Thus, it was significantly reduced in HOM and HET animals compared to the WT. Again, heroin treatment did not produce differences in locomotion between the genotypes. MDMA induced conditioned taste aversion revealed only a main effect of dose with robust conditioned taste aversion for both drug doses, although a trend indicated that HOM animals may have heightened sensitivity to MDMA. However, heroin treatment failed to produce a conditioned taste aversion effect in any of the groups regardless of dose. Beyond the aforementioned behavioural experiments striatal brain tissue from the animals that had previously self-administered MDMA or heroin was analysed via quantitative reverse transcription polymerase chain reaction; five targets were evaluated to quantify drug induced changes in brain derived neurotrophic factor gene expression (BDNF). Several BDNF isoforms (total BDNF, BDNF III and BDNF IV) were significantly increased in animals that had selfadministered MDMA; this effect was true across HOM, HET and WT subjects. Comparatively, animals that had self-administered heroin did not show a difference in BDNF expression compared to untreated control animals.

This suite of experiments provides insight into the influence of a compromised serotonergic system on the development of drug addiction. That is, while reduced SERT function does not appear to augment the addictive properties of drugs like heroin there is reason to suspect that it does confer additional susceptibility to developing addiction to drugs like MDMA, highlighting the hypothesis that different classes of addictive substances act through different neurobiological pathways. 


\section{Acknowledgements}

I would like to gratefully acknowledge the guidance, support and encouragement of my primary doctoral supervisor, Professor Bart Ellenbroek. This thanks extends also to my secondary supervisor, Professor David Harper. From the start these two brilliant scientists have supported and challenged me; they've helped me to extend my experience and expertise as I've grown as an investigator and professional.

I would like to thank the full Cognitive Behavioural Neuroscience laboratory group particularly Professor Susan Schenk for advice regarding drug self-administration. For technical advice and training in a variety of laboratory techniques I would like to thank Dr. Katie Brennan and Dr. Joyce Colussi-Mas - their help along the way was invaluable. I would also like to thank Uta Waterhouse and Sophie Muller for their help with specific projects. Lastly, I would like to thank the animal technicians, Richard Moore, Peter Vancompernolle and Michael Roberts for their support and help in the everyday care of the animals in our laboratory.

Additionally, I would to thank Dr. Darren Day for supporting the collaboration between our labs that allowed me to learn the molecular analytical technique, quantitative reverse transcription polymerase chain reaction. This thanks also extends to the students working in his lab who helped me with practical issues over many months of work; particularly, Varun Venkatesh.

On a personal note I would like to extend my biggest thanks to my husband, Tim, for his unwavering support, encouragement and help through- 
out the entire PhD program and in every aspect of our lives. He encouraged me to push myself, to not be so terribly hard on myself and to pursue ambitious goals both inside and outside the laboratory.

I must extend many thanks to both of our families for their continued support throughout this process. The packages filled with gifts and treats, the phone calls and Skype chats have made me feel that they are not so far away.

Lastly, I would like to thank the Vic Books cafe and Red Bull energy drinks for caffeine support that kept me going throughout many long term experiments. 


\section{Contents}

1 General Introduction 1

1.1 Drug Addiction . . . . . . . . . . . . . . . . 1

1.1.1 Costs Associated with Drug Addiction . . . . . . . 2

1.1.2 Terminology . . . . . . . . . . . . . 3

1.1.3 Behavioural Changes that Define Drug Addiction . . 3

1.1.4 Neurochemical Effects that Coincide with Behavioural

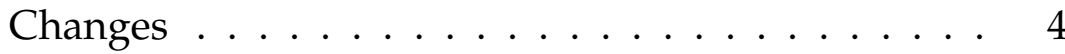

1.1.5 Transition to Drug Addiction . . . . . . . . . . 7

1.1.6 Theories of Drug Addiction . . . . . . . . . . . . . 9

1.2 Individual Differences . . . . . . . . . . . . . . . 10

1.2.1 The Serotonin Transporter (SERT) . . . . . . . . . . 11

1.2.2 The Serotonin Transporter Gene (SLC64A) and Promoter Region . . . . . . . . . . . . . . 11

1.2.3 Human Studies of the SERT . . . . . . . . . . . . . . 12

1.3 Modelling Individual Differences in the Laboratory . . . . . 18

1.3.1 Serotonin Transporter Knockout Preclinical Model . . 18

1.3.2 Point Mutation in the SLC6A4 Gene . . . . . . . . . 19

1.4 Drugs of Abuse . . . . . . . . . . . . . . . . . 20

1.4.1 3,4-methylenedioxymethamphetamine, MDMA, "Ec-

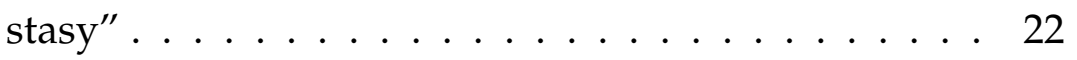

1.4.2 (5 $5,6 \alpha)-7,8$-Didehydro-4,5epoxy-17-methylmorphinan3,6-diol diacetate (ester), Heroin, diamorphine . . . . 26 
1.5 Molecular Analysis . . . . . . . . . . . . . . . . . . . 30

1.5.1 Brain Derived Neurotrophic Factor (BDNF) . . . . . . 31

1.5.2 BDNF and Neuropsychiatric Illness . . . . . . . . 31

1.6 Brief Outline of Behavioural and Molecular Experiments . . 32

2 Novelty Suppressed Feeding and

Drug Self-Administration $\quad 35$

2.1 Introduction . . . . . . . . . . . . . . . 35

2.1 .1 Predictions . . . . . . . . . . . . . . . . . . . 38

2.2 Methods . . . . . . . . . . . . . . . . 38

2.2.1 Novelty Suppressed Feeding _ . . . . . . . . . . . 38

2.2.2 Drug Self-Administration . . . . . . . . . . . . . . 39

2.3 Results . . . . . . . . . . . . . . . . . 43

2.3 .1 MDMA . . . . . . . . . . . . . . 43

2.3 .2 Heroin . . . . . . . . . . . . . . . . . . . 49

2.4 Discussion . . . . . . . . . . . . . . . . 55

3 Locomotor Activity and Conditioned

Taste Aversion $\quad 65$

3.1 Introduction . . . . . . . . . . . . . . . . 65

3.1 .1 Predictions . . . . . . . . . . . . . . 67

3.2 Methods . . . . . . . . . . . . . . . . 68

3.2.1 Locomotor Activity . . . . . . . . . . . . . . . . 68

3.2.2 Conditioned Taste Aversion . . . . . . . . . . . . . . . 69

3.3 Results . . . . . . . . . . . . . . . . . . 71

3.3.1 Locomotor Activity . . . . . . . . . . . . . . . 71

3.3.2 Conditioned Taste Aversion . . . . . . . . . . . . . 74

3.4 Discussion . . . . . . . . . . . . . . . . . . 77

4 Quantitative Reverse Transcription Polymerase Chain Reaction 83

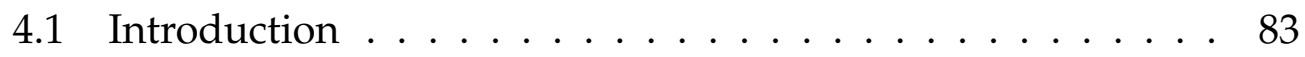

$4.1 .1 \quad$ Predictions . . . . . . . . . . . . . . . . . . 87 
4.2 Methods . . . . . . . . . . . . . . . . . . . 87

4.2.1 Quantitative Reverse Transcription Polymerase Chain Reaction . . . . . . . . . . . . . . . 88 87

4.3 Results . . . . . . . . . . . . . . . . . . . 96

4.3.1 Average Housekeeping Genes . . . . . . . . . . . . 96

4.3.2 Total BDNF . . . . . . . . . . . . . . . . . . 97

4.3.3 BDNF transcript III . . . . . . . . . . . . . . . . . . 99

4.3.4 BDNF transcript IV . . . . . . . . . . . . . . . . . . . . 101

4.3.5 BDNF transcript VI . . . . . . . . . . . . . . . . 103

4.3.6 BDNF transcript IXa . . . . . . . . . . . . . . . 106

4.4 Discussion . . . . . . . . . . . . . . . . . . . . . 108

5 General Discussion 113

5.1 Rationale . . . . . . . . . . . . . . . . . . . . . . . . . . 113

5.2 Brief Summary . . . . . . . . . . . . . . . . . . . . . . 113

5.3 Experimental Significance . . . . . . . . . . . . . . . . . 114

5.4 Limitations . . . . . . . . . . . . . . . . . . . . . . . . 120

5.4.1 Novelty Suppressed Feeding . . . . . . . . . . . . . 121

5.4 .2 Drug Self-Administration . . . . . . . . . . . . 122

5.4 .3 Locomotor Activity . . . . . . . . . . . . . . . . . . 124

5.4.4 Conditioned Taste Aversion . . . . . . . . . . . . . . . 125

5.4.5 Quantitative Reverse Transcription Polymerase Chain Reaction . . . . . . . . . . . . . . . 126

5.4.6 Translating Animal Outcomes to Humans . . . . . . . 127

5.5 Future Directions . . . . . . . . . . . . . . . . . . . 128

5.5.1 Microdialysis and In Vivo Voltammetry . . . . . . . 128

5.5.2 Ambiguous Cue Interpretation . . . . . . . . . . . . . 129

5.5.3 Emulsion PCR . . . . . . . . . . . . . . . . . . . . . . 130

5.6 Final Conclusion . . . . . . . . . . . . . . . . . . . . . 130

A Supplemental qRT-PCR Analysis 133

A.1 Section 1: Housekeeping Genes . . . . . . . . . . . . . . 133 
viii

A.2 Section 2: Total BDNF . . . . . . . . . . . . . . . 138

A.3 Section 3: BDNF Transcript III . . . . . . . . . . . . . . 142

A.4 Section 4: BDNF Transcript IV . . . . . . . . . . . . . 147

A.5 Section 5: BDNF Transcript VI . . . . . . . . . . . . . . 151

A.6 Section 6: BDNF Transcript IXa . . . . . . . . . . . . . 156 


\section{List of Figures}

1.1 The dopaminergic system. . . . . . . . . . . . . 5

1.2 The serotonergic system. . . . . . . . . . . . 7

2.1 Novelty suppressed feeding (prior to MDMA self-administration) . . . . . . . . . . . . . . . 4 44

2.2 Acquisition responding for MDMA self-administration . . . 44

2.3 Maintenance responding for MDMA self-administration . . 46

2.4 Progressive Ratio responding for MDMA self-administration 48

2.5 Novelty suppressed feeding (prior to heroin self-administration) ........................ 50

2.6 Initial drug intake for heroin self-administration . . . . . . 51

2.7 Maintenance responding for heroin self-administration . . . 52

2.8 Progressive Ratio responding for heroin self-administration 53

2.9 Novelty suppressed feeding plotted against average heroin self-administration (Maintenance) . . . . . . . . . . 54

2.10 Novelty suppressed feeding plotted against average heroin self-administration (Progressive Ratio) . . . . . . . . . . . . 54

3.1 MDMA locomotor activity . . . . . . . . . . . 72

3.2 Heroin locomotor activity . . . . . . . . . . . . 74

3.3 MDMA conditioned taste aversion . . . . . . . . . . 75

3.4 Heroin conditioned taste aversion . . . . . . . . . . 77

4.1 Exponential amplification of representative samples . . . . . 92 
4.2 Melt curve analysis of representative samples . . . . . . . . 93

4.3 Relative housekeeping gene expression . . . . . . . . . . 96

4.4 Relative gene expression of total BDNF against Cyclophilin A 98

4.5 Relative gene expression of total BDNF against HPRT . . . . 98

4.6 Relative gene expression of BDNF transcript III against $\mathrm{Cy}$ clophilin A . . . . . . . . . . . . . . . . 100

4.7 Relative gene expression of BDNF transcript III against HPRT101

4.8 Relative gene expression of BDNF transcript IV against $\mathrm{Cy}$ clophilin A . . . . . . . . . . . . . . . . . 102

4.9 Relative gene expression of BDNF transcript IV against HPRT103

4.10 Relative gene expression of BDNF transcript VI against $\mathrm{Cy}$ clophilin A . . . . . . . . . . . . . . . . . . . . 104

4.11 Relative gene expression of BDNF transcript VI against HPRT105

4.12 Relative gene expression of BDNF transcript IXa against $\mathrm{Cy}$ clophilin A . . . . . . . . . . . . . . . 107

4.13 Relative gene expression of BDNF transcript IXa against HPRT107

A.1 Relative gene expression of HPRT against Cyclophilin A $\left(\Delta C_{\mathrm{T}}\right.$ values $) \ldots \ldots \ldots \ldots \ldots \ldots$

A.2 Relative gene expression of HPRT against Cyclophilin A

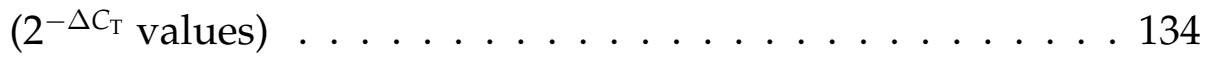

A.3 Relative gene expression of total BDNF against Cyclophilin $\mathrm{A}\left(\Delta C_{\mathrm{T}}\right.$ values $) \ldots \ldots \ldots \ldots \ldots$

A.4 Relative gene expression of total BDNF against HPRT ( $\Delta C_{\mathrm{T}}$ values) . . . . . . . . . . . . . . 140

A.5 Relative gene expression of total BDNF against Cyclophilin

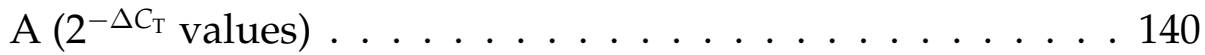

A.6 Relative gene expression of total BDNF against HPRT $\left(2^{-\Delta C_{T}}\right.$ values $\ldots \ldots \ldots$. . . . . . . . . . . . . . 142

A.7 Relative gene expression of BDNF transcript III against $\mathrm{Cy}$ clophilin A $\left(\Delta C_{\mathrm{T}}\right.$ values $) \ldots \ldots . \ldots 143$ 
A.8 Relative gene expression of BDNF transcript III against HPRT $\left(\Delta C_{\mathrm{T}}\right.$ values $) \ldots \ldots \ldots \ldots \ldots \ldots$

A.9 Relative gene expression of BDNF transcript III against $\mathrm{Cy}$ -

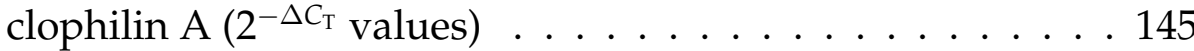

A.10 Relative gene expression of BDNF transcript III against HPRT $\left(2^{\left.-\Delta C_{\mathrm{T}} \text { values }\right) \ldots \ldots \ldots \ldots \ldots \ldots}\right.$

A.11 Relative gene expression of BDNF transcript IV against $\mathrm{Cy}$ -

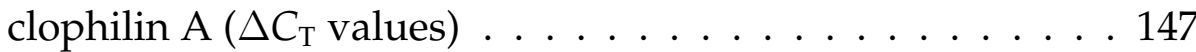

A.12 Relative gene expression of BDNF transcript IV against HPRT $\left(\Delta C_{\mathrm{T}}\right.$ values $) \ldots \ldots \ldots \ldots \ldots \ldots$

A.13 Relative gene expression of BDNF transcript IV against $\mathrm{Cy}$ -

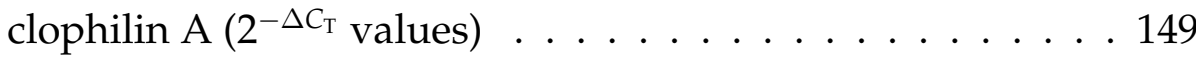

A.14 Relative gene expression of BDNF transcript IV against HPRT $\left(2^{-\Delta C_{\mathrm{T}}}\right.$ values $) \ldots \ldots \ldots \ldots \ldots \ldots \ldots$

A.15 Relative gene expression of BDNF transcript VI against $\mathrm{Cy}-$ clophilin A $\left(\Delta C_{\mathrm{T}}\right.$ values $) \ldots \ldots . \ldots . \ldots . . \ldots 152$

A.16 Relative gene expression of BDNF transcript VI against HPRT $\left(\Delta C_{\mathrm{T}}\right.$ values $) \ldots \ldots \ldots \ldots \ldots \ldots \ldots \ldots$

A.17 Relative gene expression of BDNF transcript VI against $\mathrm{Cy}$ -

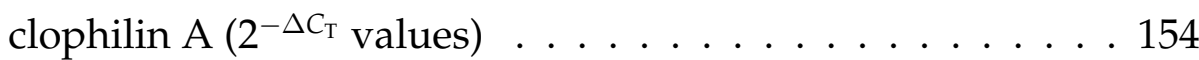

A.18 Relative gene expression of BDNF transcript VI against HPRT $\left(2^{-\Delta C_{\mathrm{T}}}\right.$ values $) \ldots \ldots \ldots \ldots \ldots$. . . . . . . . . . 154

A.19 Relative gene expression of BDNF transcript IXa against $\mathrm{Cy}$ clophilin A $\left(\Delta C_{\mathrm{T}}\right.$ values $) \ldots \ldots \ldots$. . . . . . . . 156

A.20 Relative gene expression of BDNF transcript IXa against HPRT $\left(\Delta C_{\mathrm{T}}\right.$ values $) \ldots \ldots \ldots \ldots \ldots \ldots$

A.21 Relative gene expression of BDNF transcript IXa against $\mathrm{Cy}-$

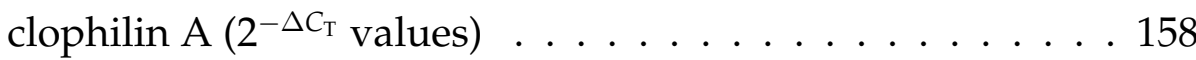

A.22 Relative gene expression of BDNF transcript IXa against HPRT $\left(2^{\left.-\Delta C_{\mathrm{T}} \text { values }\right) \ldots \ldots \ldots \ldots \ldots} 160 \ldots \ldots \ldots\right.$ 


\section{List of Tables}

1.1 Human Studies of SERT and Drug Addiction. . . . . . . . . 15

1.2 Differential aspects of psychostimulants and opioids . . . . . 21

1.3 Relative drug affinity for monoamine transporters . . . . . . 26

1.4 Relative reverse transport for monoamine transporters . . . 26

2.1 Acquisition responding for MDMA self-administration: Post hoc comparisons . . . . . . . . . . . . 45

2.2 Maintenance responding for MDMA self-administration: Post hoc comparisons . . . . . . . . . . . 47

2.3 Progressive ratio responding for MDMA self-administration: Post hoc comparisons . . . . . . . . . . . . . . . . . . . 48

2.4 Novelty Suppressed Feeding (prior to heroin self-administration): Post hoc comparisons . . . . . . . . . . . . . 50

3.1 MDMA locomotor activity: Post hoc genotype comparisons 73

3.2 MDMA locomotor activity: Post hoc drug dose comparisons 73

3.3 MDMA conditioned taste aversion: Post hoc genotype comparisons ...................... 76

3.4 MDMA conditioned taste aversion: Post hoc drug dose com-

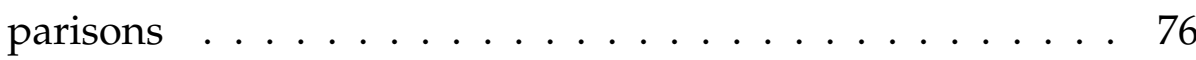

4.1 Reverse transcription reaction information: Component concentrations and volumes. . . . . . . . . . . . . . . . 90

4.2 Primer sequences for qRT-PCR analysis . . . . . . . . . . . 94 
5.1 Specific 5-HT receptor interactions with psychostimulants and opioids . . . . . . . . . . . . . . . 118

A.1 Comparison of housekeeping gene expression: HOM subjects across treatment groups. . . . . . . . . . . . 135

A.2 Comparison of housekeeping gene expression: HET subjects across treatment groups. . . . . . . . . . . . 136

A.3 Comparison of housekeeping gene expression: WT subjects across treatment groups. . . . . . . . . . . . . 137

A.4 $\Delta C_{\mathrm{T}}$ values for total BDNF against Cyclophilin $\mathrm{A}(\mathrm{a})$ and HPRT (b). . . . . . . . . . . . . . . . . . . . . . . 139

A.5 $2^{-\Delta C_{\mathrm{T}}}$ values for total BDNF against Cyclophilin A (a) and HPRT (b). . . . . . . . . . . . . . . . . . . . . . . 141

A.6 $\Delta C_{\mathrm{T}}$ values for BDNF transcript III against Cyclophilin A (a) and HPRT (b). . . . . . . . . . . . . . . . . . . . . . 144

A.7 $2^{-\Delta C_{\mathrm{T}}}$ values for BDNF transcript III against Cyclophilin A (a) and HPRT (b). . . . . . . . . . . . . . . . . 146

A.8 $\Delta C_{\mathrm{T}}$ values for BDNF transcript IV against Cyclophilin A (a) and HPRT (b). . . . . . . . . . . . . . . . . . . . . 148

A.9 $2^{-\Delta C_{\mathrm{T}}}$ values for BDNF transcript IV against Cyclophilin A (a) and HPRT (b). . . . . . . . . . . . . . . . . . . . . . 150

A.10 $\Delta C_{\mathrm{T}}$ values for BDNF transcript VI against Cyclophilin A

(a) and HPRT (b). . . . . . . . . . . . . . . . . . . 153

A.11 $2^{-\Delta C_{\mathrm{T}}}$ values for BDNF transcript VI against Cyclophilin A

(a) and HPRT (b). . . . . . . . . . . . . . . . . . . . . 155

A.12 $\Delta C_{\mathrm{T}}$ values for BDNF transcript IXa against Cyclophilin A

(a) and HPRT (b). . . . . . . . . . . . . . . . . 157

A.13 Converted $2^{-\Delta C_{T}}$ values for BDNF transcript IXa against $C y-$ clophilin A (a) and HPRT (b). . . . . . . . . . . . . . . . . . 159 


\section{Chapter 1}

\section{General Introduction}

\subsection{Drug Addiction}

When you can stop you don't want to, and when you want to stop, you can't... -Luke Davies

The development of addictive behaviours in response to psychoactive drugs is characterised by a variety of behavioural and physiological changes in the individual. In basic terms drug addiction is a chronic relapsing psychiatric disorder. It is important to note that many individuals who use drugs of abuse do not go on to develop the compulsive behaviour that defines drug addiction. However, there is a subset of individuals who make that "transition to addiction" and manifest the constellation of symptoms set forth in the Diagnostic and Statistical Manual of Mental Disorders (DSM-5) (American Psychiatric Association, 2013). A better understanding of this group of individuals represents an incredibly important area of research that will serve as a foundation for developing future curative treatments for humans. 


\subsubsection{Costs Associated with Drug Addiction}

Drug addiction represents a considerable burden for families, society and the individual. In addition to an impact on welfare, like disruptions in family relationships and vocational pursuits, drug addiction comes at a high financial cost to those affected by it. According to the World Drug Report 2014, of the 243 million people (adults) who use drugs of abuse, about $11 \%$ or 27 million represent "addicts". And of these problem drug users only one in six seeks out treatment each year. However, a change in treatment demand is emerging worldwide; for example, the number of individuals seeking treatment for cannabis use is on the rise around the world. Whereas effective opioid treatment is being sought in Eastern Europe and parts of Asia and treatment demand for amphetamine like stimulant use is increasing in Oceania. Despite these changes there remains a vast number of individuals who do not receive the help that they need and consequently there is considerable drug related loss of life. Loss of life, primarily due to drug overdose, numbers approximately 183,000 individuals annually. And while often left unreported there exists a group of individuals who survives one or more drug overdoses and is left with lifelong disability and compromised health (United Nations Office on Drugs and Crime, 2014). Premature death and reduced quality of life due to disability where evaluated in a 2013 study that assessed the global disease burden associated with drug addiction. They found that, worldwide, drug addiction led to a loss of 3.6 million years of life due to premature death (Degenhardt et al., 2013). Additionally, 16.4 million years of life were lost due to disability associated with drug addiction.

In New Zealand alone the welfare costs, including loss of life due to premature death and reduced quality of life associated with disability, total NZD \$2 billion with total costs of drug addiction reaching NZD \$6.5 billion annually. This figure is staggering as it approximates the gross domestic product of the New Zealand agricultural or finance industry (NZD \$6.7 and NZD \$ 7.0 billion, respectively) (Slack, Nana, Webster, Stokes, \& 
$\mathrm{Wu}, 2009)$.

\subsubsection{Terminology}

The term "drug addiction" is specified as substance use disorder in the DSM-5 (formerly, substance dependence). For purposes of clarity, throughout the whole of this thesis, I will use the term "addiction" or "drug addiction" to refer to the symptoms that are defined as substance use disorder by the DSM-5. While many researchers argue the appropriateness and accuracy of these terms I will use "addiction" or "drug addiction" because they are less likely to be confused with physical dependence that can result when individuals use drugs of abuse (Koob \& Volkow, 2010). Importantly, physical dependence is not necessarily a hallmark of drug addiction and therefore I will avoid using the word "dependence" in this document.

\subsubsection{Behavioural Changes that Define Drug Addiction}

The behavioural changes that accompany drug addiction, according to the DSM-5, include difficulty in limiting use of the drug. That is, even a wellintentioned individual tends to fail when attempts are made to decrease drug intake. This, in turn, leads an individual to use the substance for longer periods of time than he/ she originally intended. Over time individuals are increasingly motivated to obtain the drug and put in a considerable amount of time and financial resources in order to obtain it. This motivation is manifested when addicted individuals prioritise his/her pursuit of the drug while sacrificing important personal and vocational responsibilities. Additionally, individuals continue to use the drug despite negative consequences; therefore, even if an individual knows that his/her drug use is detrimental to mental and physical health this information is not sufficient to deter the individual from using. While the definition of drug addiction has evolved over many years tolerance and withdrawal remain an important part of how this disorder is characterised. Tolerance 
develops over time as an individual consumes increasing amounts of the drug and whereby the subjective effects begin to wane. Withdrawal occurs when an individual ceases to take the drug and experiences a cascade of deleterious effects that differ depending on the type of substance taken (American Psychiatric Association, 2013; West, 1994). In addition, even though relapse is not part of the aforementioned formal definition is it perhaps the most serious issue surrounding drug addiction. That is, individuals are at high risk for relapse even after relatively long periods, months or years, of abstinence from the drug (Deroche-Gamonet \& Piazza, 2010; Koob, Lloyd, \& Mason, 2009; Milton \& Everitt, 2012).

\subsubsection{Neurochemical Effects that Coincide with Behavioural Changes}

While drug addiction can technically be defined in terms of behavioural changes there exists an equally important series of neurochemical effects that occur as an individual develops addictive behaviour (Nestler, 2001). The neurochemical basis of addiction is contingent upon activation of the mesolimbic dopamine system; dopamine release is generally thought to underlie the rewarding properties of drugs of abuse (Badiani, Belin, Epstein, Calu, \& Shaham, 2011; Haber \& Knutson, 2010; Koob et al., 2009).

The mesolimbic dopamine system constitutes a major projection that connects the midbrain with the more rostral limbic and forebrain structures. Projections from the ventral tegmental area (midbrain) terminate in the nucleus accumbens, frontal cortex, amygdala and hippocampus (all structures located in the forebrain) (see Figure 1.1 (Kruk \& Pycock, 1993b)) (Haber \& Knutson, 2010; Iversen \& Iversen, 2007; Koob, Sanna, \& Bloom, 1998; Koob \& Volkow, 2010; Meyer, 2005).

Consequently, when dopaminergic neurons in the ventral tegmental area are stimulated dopamine is released in the target structures (e.g. nucleus accumbens) (Pettit \& Justice, 1989). The ventral tegmental bundle 


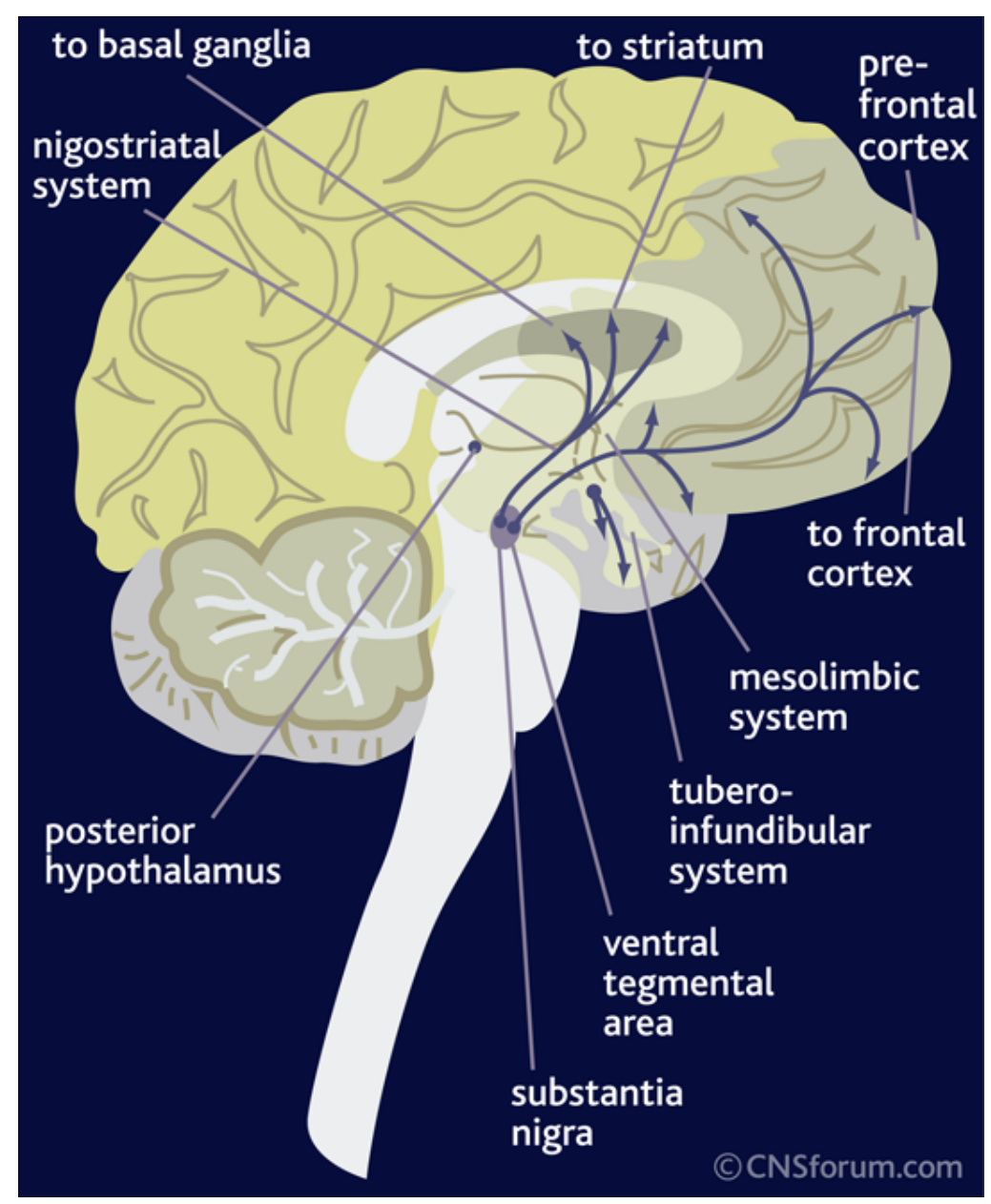

Figure 1.1: The dopaminergic system.

and its forebrain projections represent an important component of the reward system. Early work investigating the mechanisms of reward demonstrated that rats implanted with electrodes actively pressed a lever to deliver electrical current directly into this portion of the brain (Crow, 1972; Olds \& Milner, 1954). These seminal studies and a plethora of additional research since then have supported the overarching idea that dopamine represents a part of the chemical substrate that underlies the initial response to drugs of abuse (Everitt et al., 2008; Koob et al., 1998; Koob \& Volkow, 2010; Nestler, 1996, 2001). 
While a dopamine-mediated "reward circuit" is widely accepted recent research has demonstrated the integral role of the neurotransmitter serotonin (5-HT) in reward and the development and maintenance of addictive behaviours (Hayes \& Greenshaw, 2011; Kirby, Zeeb, \& Winstanley, 2011; Mueller \& Homberg, 2015; Nonkes, Van Bussel, Verheij, \& Homberg, 2011; Sora et al., 2001).

The serotonergic system originates in the rostral and caudal raphe nuclei located in the midbrain and brainstem, respectively. The rostral bundle comprises the caudal linear nucleus as well as the dorsal and median raphe nuclei. These neurons send projections to the limbic system as well as a variety of forebrain structures like the nucleus accumbens, ventral tegmental area, hippocampus, amygdala and prefrontal cortex. The caudal bundle includes the magnus, pallidus and obscurus raphe nuclei and sends projections to the spinal cord (see Figure 1.2 (Kruk \& Pycock, 1993a)) (Gaspar \& Lillesaar, 2012; Hayes \& Greenshaw, 2011; Hornung, 2003; Kranz, Kasper, \& Lanzenberger, 2010; Tork, 1990).

To date there is a multitude of studies investigating the disrupted serotonergic neurotransmission that accompanies psychiatric disorders like depression or anxiety (Hayes \& Greenshaw, 2011). Disrupted serotonin neurotransmission is a key factor in the pathology underlying these disorders and they are often treated with pharmaceutical agents that specifically target serotonergic neurotransmission allowing serotonin additional time to act on target cells (Reinhold, Mandos, Rickels, \& Lohoff, 2011; Taurines, Gerlach, Warnke, Thome, \& Wewetzer, 2011). More recently focus has shifted to trying to understand the role that serotonin neurotransmission plays in the development and maintenance of addictive behaviours. Like dopamine, serotonin is an integral player in the neurochemical changes that accompany a diagnosis of drug addiction. For example, some drugs of abuse act on the serotonergic system by triggering release of 5-HT in several brain regions but inhibit 5-HT release when an individual is experiencing withdrawal; this contributes to the negative 


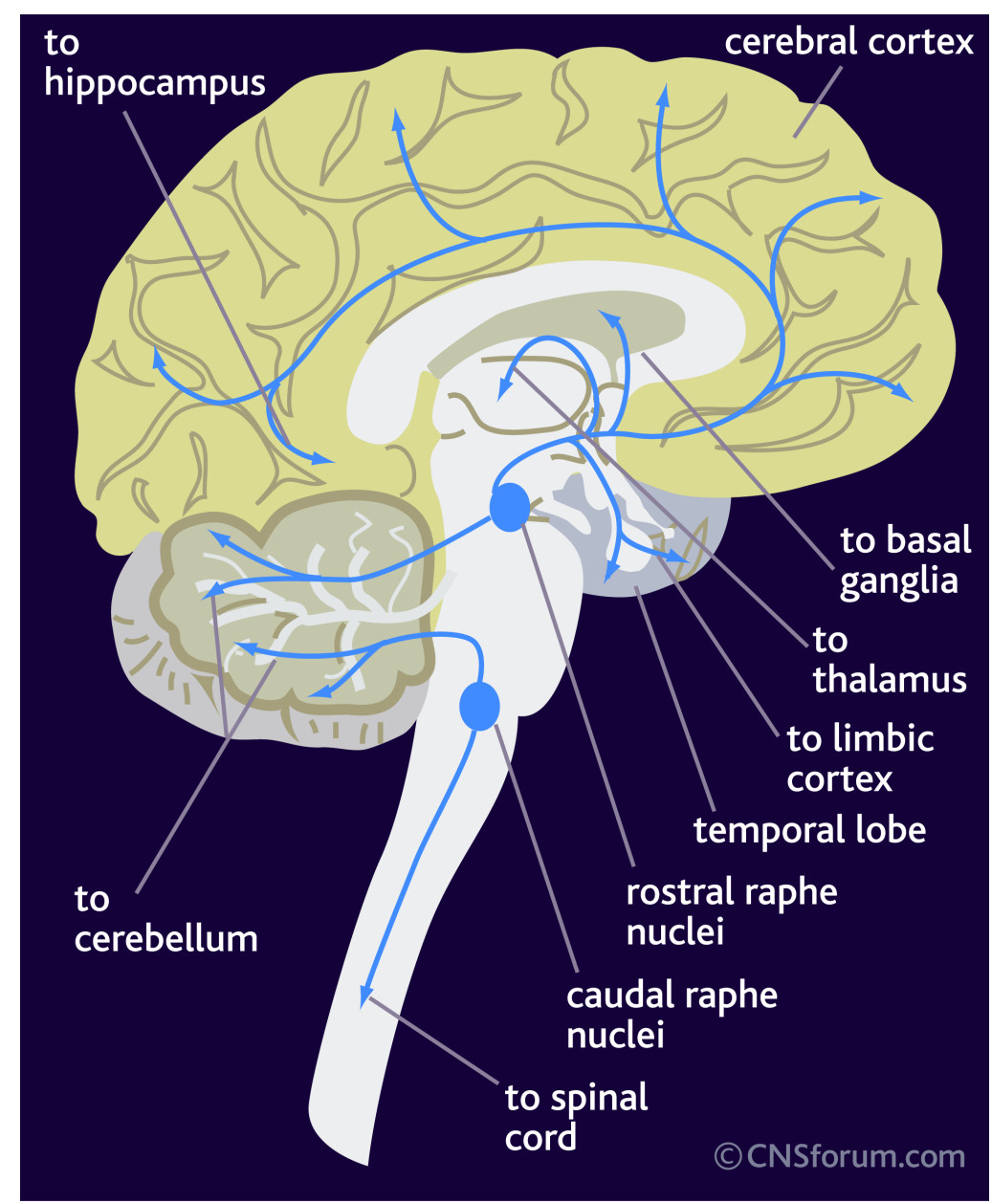

Figure 1.2: The serotonergic system.

withdrawal symptoms and can lead to escalated intake or relapse (Kirby et al., 2011; Nonkes et al., 2011).

\subsubsection{Transition to Drug Addiction}

Interestingly, only a small percentage (15-35\%) of individuals who use drugs of abuse go on to develop the compulsive behaviour that defines drug addiction (Badiani et al., 2011; Deroche-Gamonet \& Piazza, 2010; United Nations Office on Drugs and Crime, 2014). This suggests that there 
is a difference between individuals who go on to develop addictive behaviour and those that do not. Researchers refer to this difference among drug users as a "transition to addiction". Drug users begin using the substance and continue to do so initially because it possesses rewarding properties; dopaminergic activation in the ventral portions of the striatum including the nucleus accumbens underlie these enjoyable subjective experiences. Described in terms of behaviour drug use initially can be seen as an action and the outcome that follows it; therefore, individuals initially continue taking a drug because they are pursuing the outcome (i.e. "high") that follows drug intake. However, after continued use the drug influences neural plasticity; it does this by first shifting dopaminergic activation from the ventral to more dorsal portions of the striatum. Second, a critical change occurs within the dorsal striatum itself; control mediated by the dorsomedial striatum is ultimately shifted more laterally within the structure. These changes underlie the transition from pursuing an outcome ("high") to a largely automatic habitual pattern of drug taking. And this is why individuals diagnosed with drug addiction continue to use the drug long after the enjoyable subjective experiences have waned due to the development of tolerance (Everitt, 2014; Everitt et al., 2008). Additionally, while initial drug use is associated with transient changes in synaptic plasticity (long term depression) in the nucleus accumbens these changes become permanent as drug use increases. This inflexibility in the neural connections in the dopaminergic pathways may underlie the "transition to addiction" (Kasanetz et al., 2010). These neural changes represent an integral component in the "transition to addiction" and constitute the biological substrate that underlies the behavioural changes seen in individuals given a diagnosis of drug addiction (Ahmed, 1998). 


\subsubsection{Theories of Drug Addiction}

Theories of drug addiction posit that changes in drug effects may explain why some individuals take drugs compulsively. Some research suggests that addictive behaviour is driven by changes in the effect of drugs; that is, compulsive drug use is driven by the rewarding properties of drugs of abuse. Additional research posits that individuals continue to take drugs in order to avoid the negative consequences of withdrawal. That is, individuals may develop increased sensitivity to either the positive properties of drugs of abuse or to the negative effects and this sensitivity underlies compulsive drug taking behaviour.

The incentive-sensitisation theory put forth by Robinson and Berridge (1993) proposes that compulsive drug intake produces a sensitised neural "reward" network where the reinforcing effects of the drug are pathologically enhanced. This sensitisation enhances the salience of drug related stimuli making them more attractive and increasing the likelihood that the user will consume the drug. With a heightened drug effect and increasingly powerful salience of drug related stimuli drug taking behaviour is increasingly reinforced (Robinson \& Berridge, 1993, 2001). While there is compelling support for incentive-sensitisation it is important to acknowledge research findings from other groups that have investigated sensitisation to drugs of abuse. In both conditioned place preference and drug self-administration paradigms researchers have demonstrated a dissociation between sensitisation and subsequent addictive behaviours (Ahmed, 2010; Brown, Short, \& Lawrence, 2010).

In contrast to incentive-sensitisation others argue that individuals make the transition to addiction because the negative consequences associated with drug withdrawal increase over time. Koob et al. (2009) suggested that initially drug taking is driven by the desire to experience the positive subjective effects of the drug (e.g. euphoria). However, after continued use and concurrent with the transition to drug addiction the negative consequences of withdrawal (e.g. anxiety, irritability and dysphoria) increase 
driving an escalation in drug taking. This view is supported by the fact that individuals take increasing amounts of drug over time and exhibit neurobiological changes as drug intake continues (Koob, 1992; Koob et al., 2009, 1998).

Both explanations suggest that there are inherent differences within individuals themselves. That is, because only a small percentage of individuals who use drugs of abuse go on to develop addictive behaviours this has led researchers to ask the question: Are there genetic differences amongst individuals that might represent risk factors in the development of drug addiction? (Deroche-Gamonet \& Piazza, 2010). Studies in animals demonstrate similar patterns; that is, in groups of rats only a small percentage ( $\sim 20 \%$ ) develop addictive like behaviours (Deroche-Gamonet, Belin, \& Piazza, 2004; Pelloux, Dilleen, Economidou, Theobald, \& Everitt, 2012). Historical research has focused on understanding addiction in light of its influences on drug effects as well as understanding the role of genetic vulnerability. More recent research has shifted focus to evaluating the contribution of specific genes in the development of drug addiction.

\subsection{Individual Differences}

As mentioned above researchers have started to investigate individual genetic differences to understand why some individuals who use a drug of abuse become addicted while others do not (Deroche-Gamonet \& Piazza, 2010; Lesch \& Gutknecht, 2005). One type of genetic variation, that is a polymorphism in the promoter region of the serotonin transporter gene, has been implicated in the development of drug addiction and may represent an important point of focus in understanding how genetic variability influences the probability of developing addictive behaviours (Lesch \& Gutknecht, 2005; Murphy et al., 2001). 


\subsubsection{The Serotonin Transporter (SERT)}

The serotonin transporter (SERT) is a presynaptic protein located on, among others, cerebral serotonin neurons. When a serotonergic neuron is stimulated it releases serotonin into the synaptic cleft where it acts on the 5-HT receptors of adjacent cells. The vast majority of the serotonin left in the synapse is taken back up into the presynaptic terminal via the SERT. The serotonin is then repackaged and can be used at a later time. It is important to note that serotonin reuptake via the SERT is the primary mechanism that stops serotonin action in the synapse and therefore plays a crucial role in overall serotonin neurotransmission in the brain (Kenna et al., 2012; Lesch et al., 1996). Additionally, the SERT represents a target for certain anxiolytic and antidepressant medications; they block the SERT allowing serotonin to remain in the synaptic space where it will continue to interact with adjacent cells (Kenna et al., 2012; Reinhold et al., 2011; Taurines et al., 2011).

\subsubsection{The Serotonin Transporter Gene (SLC64A) and Pro- moter Region}

The gene that codes for the SERT, SLC6A4, located on chromosome 17 in humans, is regulated by an upstream promoter region. A genetic polymorphism or variation in the DNA sequence, in the promoter region, 5HTTLPR, influences transcription of the SERT protein. Specifically, the polymorphism consists of a 44 base pair insertion or deletion which in turn yields a long (16 base pair element repeats) or short (14 base pair element repeats) allele. The long allele promotes standard protein transcription and consequently does not disrupt serotonergic neurotransmission in the brain. Conversely, the short allele results in reduced protein transcription which leads to a decreased number of SERT proteins on serotonergic neurons. With fewer SERT proteins to remove serotonin from the synapse extracellular levels of serotonin increase (Kenna et al., 2012; Lesch et al., 
1996; Su et al., 2009; Watanabe et al., 2011).

As humans have two sets of chromosomes, inheriting a single chromosome from each parent, an individual may be homozygous for the 1 allele $(1 / 1)$, homozygous for the s allele (s/s) or heterozygous $(1 / \mathrm{s})$. Following the description above individuals who are homozygous for the 1 allele $(1 / 1)$ represent those with undisrupted serotonergic reuptake as they have the greatest amount of SERT protein (100\%) compared to the other allelic combinations. Conversely, those homozygous for the s allele (s/s) have reduced serotonergic reuptake because they only have half as much SERT protein (50\%) compared to those with the 1/1 genotype. Lastly, those that are heterozygous $(1 / \mathrm{s})$ have about $75 \%$ of the SERT protein (Kenna et al., 2012; Lesch et al., 1996; Lesch \& Gutknecht, 2005).

To appreciate the impact of the SERT polymorphism it is important to understand how often it occurs within various population groups. Importantly, the incidence of the SERT polymorphism varies widely between ethnic groups. As outlined in a comprehensive meta-analysis the incidence of the $\mathrm{L}$ allele is highest in those of European and African descent (57\% and $62 \%$, respectively). Indiviuals of Mexican or Asian descent have the lowest incidences in populations studied thus far $(46 \%$ and $27 \%$, respectively) (Cao, Hudziak, \& Li, 2013).

\subsubsection{Human Studies of the SERT}

Study of this functional polymorphism is widespread and covers topics including its relationship with anxiety and mood disorders as well as its influence on the development and maintenance of addictive behaviours (Cao et al., 2013; Caspi et al., 2003; Gorwood, Batel, Ades, Hamon, \& Boni, 2000; Kenna et al., 2012; Laucht et al., 2009; Lesch et al., 1996; Merenakk et al., 2011). 


\section{SERT and Depression and Anxiety}

Researchers have shown that individuals who have reduced SERT function (one or two s alleles) tend to show greater susceptibility to developing depression (Cervilla et al., 2006; Lesch \& Gutknecht, 2005; Murphy et al., 2001).

Other groups have described the relationship between reduced SERT function and the development of anxiety indicators using a variety of personality assessments based on the five factor model of personality, Cattels personality inventory as well as a biosocial model that classifies variation in temperament. The finding that individuals with one or two copies of the $s$ allele reported higher levels of anxiety than those homozygous for the 1 allele was consistent across these measures (Lesch et al., 1996).

Additionally, research investigating the neurological substrate underlying anxiety suggests that there is a relationship between the SERT polymorphism and activity in the amygdala. That is, individuals with reduced SERT function show higher levels of neuronal activity in the amygdala (where fear is processed) than individuals with intact SERT function. This over activation of the amygdala occurs only with fearful, not neutral or positive, stimuli and is considered an abnormal fear response. With that said individuals with one or two s alleles may be more likely to develop anxious symptoms due to an overactive amygdala leaving them vulnerable when confronted with fearful environmental stimuli (Hariri et al., 2002; Heinz et al., 2005).

It is also important to note that many research groups have focused on illuminating the complex interplay between environmental factors (e.g. stress) and the SERT polymorphism (Caspi \& Moffitt, 2006). For example, Caspi et al. (2003) investigated the relationship between stress and the development of depression. What they found was a significant moderation by the SERT polymorphism; individuals with reduced SERT function (one or two copies of the s allele) were more likely to develop depression than those who were homozygous for the 1 allele. These findings implicate dis- 
rupted serotonergic neurotransmission, which occurs when an individual carries one or two s alleles, with the development of depression (Karg, Burmeister, Shedden, \& Sen, 2011; Mann, 1999).

Interestingly, van ijzendoorn et al. (2012) demonstrated that individuals who carry the $\mathrm{s} / \mathrm{s}$ and $\mathrm{s} / \mathrm{l}$ genotypes are more susceptible to the influence of negative environments (e.g. one study investigated the relationship between family risk and depression) compared to those with the $1 / 1$ genotype. They also determined that individuals who were heterozygous or homozygous for the s allele were more "open" to the influence that a positive environment might have (e.g. one study looked at the effects of responsive parenting on positive development in their children). Whereas most studies focus on the negative outcomes that may result for those with reduced SERT function this study introduces the idea that these individuals may benefit more readily from positive experiences than those with the $1 / 1$ genotype. This suggests that individuals with one or two copies of the $\mathrm{s}$ allele may be, more or less, equally open to the influence of positive and negative environmental situations (van Ijzendoorn, Belsky, \& BakermansKranenburg, 2012).

\section{SERT and Drug Addiction}

As discussed above there is a plethora of research to support the connection between reduced SERT function and anxiety and mood disorders. Interestingly, there is high comorbidity between anxiety and mood disorders and drug addiction (Grant et al., 2004; Kilpatrick et al., 2003; Regier et al., 1990). Therefore, there may be a relationship between serotonergic dysfunction (reduced SERT function) and the development of drug addiction. The following section will briefly outline the relevant research findings regarding this relationship. Please see Table 1.1 for a list of the studies discussed. They are divided into two categories: first, studies that provide support for vulnerability to engage in drug taking behaviour and the s allele and, second, those that implicate the 1 allele in drug taking behaviour. 


\begin{tabular}{|l|r|r|r|}
\hline Drug(s) & Participant & Allele & Reference \\
\hline Alcohol, heroin, cocaine & Addict & s & Enoch et al. 2011 \\
\hline Heroin & Addict & s & Gerra et al. 2004 \\
\hline Alcohol, tobacco & Healthy & s & Merenakk et al. 2011 \\
\hline Nicotine & Healthy & s & Nilsson et al. 2009 \\
\hline Alcohol & Healthy & s & Kaufman et al. 2007 \\
\hline Nicotine & Healthy & s & Gerra et al. 2005 \\
\hline Alcohol and illegal drugs & Healthy & s & Gerra et al. 2005 \\
\hline Alcohol & Healthy & s & Herman et al. 2003 \\
\hline Alcohol & Healthy & s & Herman et al. 2005 \\
\hline \hline Alcohol & Addict & 1 & Ait-Daoud et al. 2009 \\
\hline Alcohol & Addict & 1 & Kweon et al. 2005 \\
\hline Alcohol & Healthy & 1 & Laucht et al. 2009 \\
\hline Nicotine & Healthy & 1 & Ishkawa et al. 1999 \\
\hline Nicotine & Healthy & 1 & Kremer et al. 2005 \\
\hline
\end{tabular}

Table 1.1: Human Studies of SERT and Drug Addiction.

Interestingly, a portion of these studies demonstrates that reduced SERT function may confer susceptibility where individuals carrying the s allele are more likely to engage in drug taking behaviour than those that carry the 1 allele. However, other studies suggest that the 1 allele may represent a risk factor in the development of drug addiction instead of the s allele (see Table 1.1).

The aforementioned mixed results beg the question: Are there factors that can account for the discrepancies so that scientists can collate the entire body of research to inform the general topic of the SERT polymorphism and its relationship to drug addiction? While this is no straightforward task I will describe three factors that may contribute to the difficultly in understanding this complex relationship between genes and behaviour: differences in ethnicity, allelic variation and the types of drugs of abuse 
that are used.

The ethnicity of an individual may influence his/her susceptibility to develop addictive behaviours. For example, as mentioned above van Ijzendoorn et al. (2012) showed that individuals, across all ethnicities, who carry the s/s and s/l genotypes are more susceptible to the influence of negative environments compared to those with the $1 / 1$ genotype. Interestingly, they also determined that, in a Caucasian sample, individuals who were homozygous or heterozygous for the s allele were more "open" to the influence that a positive environment might have compared to Caucasian individuals who were homozygous for the 1 allele (van Ijzendoorn et al., 2012). This may suggest that the combination of certain ethnicities with specific environmental conditions could represent a risk factor in the development of addictive behaviours.

It is also important to note that across geographic regions the percentage of individuals carrying any of the allelic combinations (s/s, 1/s, 1/1) can vary significantly (Chiao \& Blizinsky, 2010). Specifically, Gelernter at al. (1997) showed that when Japanese and European American sample groups were compared those carrying the s allele comprised a much larger percentage (70-80\%) of the Japanese group compared to the European American group (40-45\%) (Gelernter, Kranzler, \& Cubells, 1997). Additionally, Noskova et al. (2008) showed that even among an all-European sample (comprised of Croats, Russians, Tatars and Bashkirs) there were significant allelic differences among the subgroups. Individuals from the Croat sample group were more likely to carry the s/s genotype than those from the other groups (Noskova et al., 2008).

The second major consideration to be addressed is the inherent complexity of human allelic variation. That is, most studies analyse genetic material from participants and place them into various subgroups: $s$ or 1 allele, or the allelic combinations $\mathrm{s} / \mathrm{s}, 1 / \mathrm{s}$ and $1 / 1$. In truth, this is the simplest way to approach the SERT polymorphism because the variation extends beyond the $s$ and 1 alleles. Kenna et al. (2012) made this point 
when discussing the complexity of the polymorphism. They discuss that the 1 allele can contain a single nucleotide polymorphism that results in an $\mathrm{L}_{\mathrm{A}}$ or an $\mathrm{L}_{\mathrm{G}}$ allele. These alleles then reflect differences in the transcriptional activity of the gene where the $\mathrm{L}_{\mathrm{G}}$ has similar functionality to the $\mathrm{s}$ allele (Kenna et al., 2012).

Previous research has described the well-known s and 1 allelic differences, that comprise 14 and 16 base pair element repeats (Heils et al., 1996; Mortensen, Thomassen, Larsen, Whittemore, \& Wiborg, 1999). However, there has been subsequent research to back the identification of three additional alleles $(18,19$, and 20 base pair element repeats) (Delbruck et al., 1997; Kunugi et al., 1997; Michaelovsky et al., 1999). Extending these studies Nakamura et al. (2000) delineated ten novel allelic variants to give a combined total of fourteen different possible human alleles (Nakamura, Ueno, Sano, \& Tanabe, 2000).

Lastly, there is a wide variety of drugs of abuse and this variability undoubtedly complicates the relationship between the SERT polymorphism and the development of drug addiction. Drugs of abuse can be separated into different groups depending on their mechanism of action. For example, when drugs like alcohol or heroin are taken they indirectly stimulate dopamine release whereas stimulant drugs directly target the dopaminergic transporter which prolongs dopamine action. Conversely, "ecstasy" (MDMA) has a different mechanism of action; MDMA blocks the SERT and moves 5-HT out of the presynaptic neuron by reverse transport in addition to acting on the dopamine transporter. Therefore, depending on a variety of individual differences as well as different drug mechanisms the relationship between the SERT polymorphism and drug addiction is highly complex (Green, Mechan, Elliott, O'Shea, \& Colado, 2003; Kreek et al., 2012).

Taken together these studies outline some of the potential issues surrounding the interpretation of research results from human studies investigating the relationship between the SERT polymorphism and the devel- 
opment of drug addiction. Therefore, to clarify some of these issues it would be worthwhile to measure SERT activity directly. This is made possible by using animal models, specifically rats and mice, that have a targeted reduction in the SERT.

\subsection{Modelling Individual Differences in the Lab- oratory}

The SERT polymorphism represents a genetic mutation of great interest to researchers who are investigating the role of genes in the development of a variety of psychiatric disorders including addiction to drugs of abuse. Because the neurochemical and behavioural changes that accompany addictive behaviours are highly complex it is essential that researchers utilise a viable and sustainable laboratory model. Like many complex physiological processes these behavioural and neurochemical changes cannot be successfully studied in vitro or directly in humans due to ethical issues surrounding use of humans in laboratory research. Therefore, a "middle ground" of sorts, animal models, is widely used in drug addiction research to investigate the complex behavioural and neurochemical changes that accompany drug intake.

\subsubsection{Serotonin Transporter Knockout Preclinical Model}

Initially, in an effort to study a reduction in the SERT in the laboratory a serotonin transporter knockout (SERT KO) mouse model was developed (Lira et al., 2003). These animals were created using a homologous recombination technique yielding homozygotes with $0 \%$ SERT function. Their behavioural phenotype has been characterised with SERT KO mice showing heightened levels of anxious and depressive behaviours when evaluated with a variety of behavioural paradigms (Fox et al., 2007; Lira et al., 2003). Additionally, several research groups have investigated the impact 


\subsection{MODELLING INDIVIDUAL DIFFERENCES IN THE}

of disrupted SERT function on brain physiology as well as in relation to the development of drug addiction (Fabre et al., 2000; Fox et al., 2007; Trigo et al., 2007).

Since then researchers have developed a genetically altered rat model: the serotonin transporter knockout rat (SERT KO). Smits et al. (2006) investigated the effectivity of a super mutagen, $N$-ethyl- $N$-nitrosourea, or ENU, to alter the genetic material of Wistar rats. Male Wister rats were treated once a week for three weeks with ENU and then mated with wild type females. The resulting offspring were processed via mutation screening to determine what point mutations had occurred in the animals. Those that had a mutation of interest were then backcrossed with wild type animals to effectively isolate the mutation (Smits et al., 2006).

\subsubsection{Point Mutation in the SLC6A4 Gene}

Of the various point mutations that resulted, six of these produced a premature stop codon leading to a truncated polypeptide chain. The premature stop codon is identified by a specific biological process that breaks down the messenger RNA and subsequently no protein is formed (Smits et al., 2006).

One of the mutations produced a premature stop codon in the gene that codes for the serotonin transporter protein, SLC6A4. In Wild type $\left(\mathrm{SERT}^{+/+}\right)$animals neither allele contains a premature stop codon and this promotes standard protein synthesis (100\% SERT protein). Conversely, both alleles in homozygous (SERT ${ }^{-/}$) animals contain a premature stop codon and lead to no protein synthesis ( $0 \%$ SERT protein). Lastly, heterozygous (SERT ${ }^{+/}$) animals have a premature stop codon in one of the two alleles; this combination promotes reduced protein synthesis $(50 \%$ SERT protein) (Homberg et al., 2007).

It is important to note that these rat genotypes do not match those of humans exactly. To our knowledge there are no humans who lack the 
SERT completely but for those who are homozygous for the s allele they would be similar to rats that are heterozygous as both of these groups have $50 \%$ of the standard amount of SERT protein. Of course, the humans with the allelic combination $1 / 1$ and the wild type rats represent those with standard serotonergic neurotransmission and can serve as an important comparison to those with reduced SERT function.

As the SERT has been implicated in a variety of psychiatric disorders the serotonin transporter knockout rat model provides a unique opportunity to systematically study a reduction in the SERT and how it might be related to the development of drug addiction. This particular model elegantly mimics reduction in the SERT that happens naturally in humans. Other laboratory models have investigated the relationship between serotonergic function and drug addiction by lesioning serotonergic neurons; however, these manipulations were conducted in adult animals (Bradbury \& Schenk, 2011). The SERT KO model animals, like humans with reduced SERT function, are born with an altered serotonergic system. This provides a more precise way to evaluate the development of drug addiction within a system that has been compromised from conception.

\subsection{Drugs of Abuse}

While it is widely accepted that drugs of abuse activate the mesolimbic dopamine system the specific mechanism of action as well as additional neurotransmitter systems involved can differ substantially between disparate classes of drugs (see Table 1.2 for a summary of the fundamental differences) (Badiani et al., 2011). To highlight the differential mechanisms of drug action the following sections will compare and contrast the psychostimulant, 3,4-methylenedioxymethamphetamine (MDMA) and the opioid, heroin. 


\begin{tabular}{|c|c|}
\hline \multicolumn{2}{|c|}{ Differential Aspects of Psychostimulants (PS) and Opioids (OP) } \\
\hline Impulsivity & $\begin{array}{l}\text { Greater impulse control and cognitive flexibility } \\
\text { deficits for PS vs OP }\end{array}$ \\
\hline References & $\begin{array}{l}\text { Ersche et al. 2005, 2006; Winstanley et al. 2009; McNa- } \\
\text { mara et al. } 2010\end{array}$ \\
\hline Mechanism of Action & $\begin{array}{l}\text { PS target monoamine transporters; OP target } \mu \text {-opioid } \\
\text { receptors }\end{array}$ \\
\hline References & $\begin{array}{l}\text { Harris \& Baldessarini, 1973; Gyling \& Wang, 1983; } \\
\text { Johnson \& North, } 1992\end{array}$ \\
\hline Neuronal Subpopulations & $\begin{array}{l}\text { PS and OP stimulate different neuronal populations in } \\
\text { the NAc and mPFC }\end{array}$ \\
\hline References & Carelli \& King, 1993; Chang et al. 1997, 1998 \\
\hline Serotonergic interactions & $\begin{array}{l}\text { Differential implication of 5-HT receptors in PS and OP } \\
\text { addiction }\end{array}$ \\
\hline References & Carboni et al. 1989; Porras et al. 2002, 2002a \\
\hline Synaptic Plasticity & $\begin{array}{l}\text { PS withdrawal promotes LTP in the } \mathrm{mPFC} \text {, OP with- } \\
\text { drawal does not }\end{array}$ \\
\hline References & $\begin{array}{l}\text { Huang et al. 2007; Van den Oever et al. 2008; Lu et al. } \\
2010\end{array}$ \\
\hline Structural Plasticity & $\begin{array}{l}\text { PS increases dendrite branching and density in NAc, } \\
\text { mPFC neurons, OP decreases dendrite complexity }\end{array}$ \\
\hline References & Robinson \& Kolb, 1997; Robinson \& Kolb, 2004 \\
\hline Behavioural Drug Effects & $\begin{array}{l}\text { PS increases approach avoidance (appetitive/aversive } \\
\text { state), whereas OP increases approach behaviour (ap- } \\
\text { petitive) }\end{array}$ \\
\hline References & $\begin{array}{l}\text { Ettenberg, 2004; Ettenberg \& Geist, 1993; Ettenberg et } \\
\text { al. 1999; Geist \& Ettenberg, } 1997\end{array}$ \\
\hline Compulsive Drug Use & $\begin{array}{l}\text { PS produce binge drug taking, whereas OP produce a } \\
\text { gradual increase in drug intake }\end{array}$ \\
\hline References & Pickens \& Harris, 1968; Weeks \& Collins, 1968 \\
\hline Relapse & $\begin{array}{l}\text { PS reinstatement is driven by few brain regions (VTA, } \\
\text { dorsal } \mathrm{mPFC}, \mathrm{NAc} \text { core) compared to OP }\end{array}$ \\
\hline References & McFarland and Laivas, 2001; Rogers and See, 2008 \\
\hline Setting of drug taking & $\begin{array}{l}\text { Enhanced PS effects in "non-resident" environment, } \\
\text { enhanced OP effects in "home" environment }\end{array}$ \\
\hline References & Caprioli et al. 2007, 2008, 2009; Spagnolo et al. 2011 \\
\hline
\end{tabular}

Table 1.2: Differential aspects of psychostimulants and opioids. 


\subsubsection{3,4-methylenedioxymethamphetamine, MDMA, "Ec- stasy"}

MDMA is a ring substituted synthetic drug that belongs to the amphetamine family; before 1985 it was not officially considered a drug of abuse nor was it included in the Schedule 1 list of substances that are deemed illegal. In 1985 the Food and Drug Administration of the United States decided that MDMA had sufficient abuse liability and therefore should be given Schedule 1 status. Before this it was a widely held belief that MDMA was inherently different from other drugs of abuse; that it was less addictive and therefore less dangerous than other highly addictive drugs like cocaine (Green et al., 2003; McDowell \& Kleber, 1994; Schenk, 2011).

\section{Acute Effects of MDMA}

When queried about the acute subjective effects experienced when taking MDMA users describe an overall feeling of "warmth", "closeness" or "connection" to those around them. MDMA has become known for this effect and was termed "ecstasy". Interestingly, the Los Angeles drug dealer who named MDMA "ecstasy" originally wanted to name it "empathy" but decided against it for fear that people wouldn't understand the term. Regardless, MDMA is now known as "ecstasy" and consistently produces feelings of warmth and connection in those who use it (McDowell \& Kleber, 1994). The positive subjective effects of "closeness" to others is said to be the result of MDMA's action on the serotonergic system. That is, MDMA blocks the SERT and moves serotonin into the synapse via reverse transport; the serotonin remains there because the SERT cannot move it back into the presynaptic cell and therefore prolongs its action on adjacent cells (Rattray, 1991).

In addition to the empathetic feelings described above MDMA also produces an overall increase in motor activity as well as increased heart 
rate and blood pressure. Other acute subjective effects of MDMA include hyperthermia, nausea, chills, bruxism (jaw clenching) and increased sexual arousal although some users report difficulty reaching orgasm (Green et al., 2003; McDowell \& Kleber, 1994; Parrott, 2002; Schenk, 2011). This constellation of symptoms has been termed "serotonin syndrome" which is directly precipitated by increased levels of extracellular serotonin that result when MDMA acts on serotonin neurons. Gillman (1999) argues that "serotonin syndrome" represents a continuum where symptoms may vary due to individual differences, drug dose and poly drug use. With increasing serotonin syndrome severity comes increased risk of death (Gillman, 1999).

\section{Chronic Effects of MDMA}

With increasing use of MDMA serotonin stores are repeatedly depleted and this leads to the development of tolerance. Where initially a smaller amount of drug was needed to induce the positive subjective effects described above after repeated use serotonin is depleted and individuals need more of the drug to achieve the "high" experienced at the onset of drug use (McDowell \& Kleber, 1994).

There is a myriad of research to support the idea that MDMA use may lead to serotonergic neuronal toxicity (Green et al., 2003; McCann \& Ricaurte, 1993; Parrott, 2002; Schenk, 2011). This toxicity involves the degradation of serotonin neuron axon terminals and is caused by the increased formation of free radicals. Free radicals are unstable groups of atoms or molecules that damage biological tissue in humans and animals; in this case the MDMA facilitates free radical damage in serotonin neurons leading to a downregulation of serotonin in several regions of the brain (e.g. forebrain and hippocampus) (Colado, O'Shea, Granados, Murray, \& Green, 1997; Fischer, Hatzidimitriou, Wlos, Katz, \& Ricaurte, 1995; Ohearn, Battaglia, Desouza, Kuhar, \& Molliver, 1988; Sharkey, McBean, \& Kelly, 1991). 
It is important to note that MDMA induced hyperthermia may influence the level of neurotoxicity and is considered a hallmark of chronic MDMA use; with increased hyperthermia comes more extreme changes in neuroregulation. Malberg and Seiden (1998) investigated the effect of varying ambient temperature on MDMA induced neurotoxicity and found that rats exposed to higher temperatures also had more severe deficits in serotonin levels compared to those that had been exposed to MDMA but at lower temperatures (Malberg \& Seiden, 1998).

To complement and extend studies done in laboratory animals researchers have started to investigate the potentially deleterious effects of MDMA in humans. There are several studies that may support the idea that MDMA causes changes in neuroregulation in humans comparable to that seen in laboratory animals. For example, even though initially MDMA was seen as a "non-addictive" substance there is now evidence to demonstrate that some users consume amounts of the drug comparable to what can cause neurotoxicity in non-human primates (Ricaurte, Delanney, Irwin, \& Langston, 1988). Additionally, Ricaurte et al. (2000) demonstrated that exposure to MDMA in non-human primates caused a permanent loss in specific serotonin neuron populations (Ricaurte, Yuan, \& McCann, 2000).

Ricaurte et al. (1990) investigated changes in the concentration of 5hydroxyindoleeactic acid (5-HIAA), a serotonin metabolite, in cerebrospinal fluid (CSF) of human participants. The measurement of 5-HIAA is often used as a determinant of serotonin neuron function with reduced function indicating a disruption in serotonin neurotransmission. This study revealed that the 5-HIAA levels in MDMA users were significantly (26\%) lower than in control participants (Ricaurte, Finnegan, Irwin, \& Langston, 1990).

Semple et al. (1999) also investigated MDMA induced effects in human using single photon emission computed tomography (SPECT) coupled with a SERT ligand that binds to SERT proteins. After binding to the SERT the ligand emits gamma radiation that is detected by the scan- 
ner. This technique shows the density of SERT proteins as they are identified by the ligand in various areas of the brain. The study demonstrated a reduced number of SERT proteins in MDMA users compared to control participants which may be evidence of a downregulation in serotonergic neurotransmission (Semple, Ebmeier, Glabus, O'Carroll, \& Johnstone, 1999). In a similar study McCann et al. (1998) used positron emission tomography (PET) coupled with a SERT ligand. They found that MDMA users had significantly lower densities of SERT proteins compared to control participants (McCann, Szabo, Scheffel, Dannals, \& Ricaurte, 1998). By investigating the living, human brain these latter two studies suggest that MDMA can induce long term changes in the serotonergic system.

Taken together these human and non-human primate studies demonstrate that MDMA may have a damaging effect on the serotonergic system. This is evidenced by the permanent loss of certain serotonin neuron populations; in addition, MDMA users show reduced levels of 5-HIAA in CSF and number of SERT proteins in the brain.

\section{MDMA Mechanism of Action}

Like other drugs of abuse MDMA increases activity in the mesolimbic dopamine pathway. However, it is important to note that MDMA also influences the serotonergic and noradrenergic neurotransmitter systems. Specifically, when MDMA is taken up by the body, usually via oral consumption of pills, it binds to the dopamine, serotonin and noradrenaline transporters (DAT, SERT and NET, respectively) and inhibits them from removing their respective neurotransmitters from of the synaptic space and effectively stopping their action. It also stimulates release of these neurotransmitters into the synapse via reverse transport; that is, the neurotransmitter that is being held in the terminal buttons is transported out of the cell through its respective transporter. While this leads to an increase in extracellular dopamine, noradrenaline and serotonin MDMA has the highest affinity for the SERT and therefore influences the serotonergic 
system more so than the other systems (Han \& Gu, 2006). This contrast is readily apparent when considering the mechanism of action in other drugs of abuse like cocaine and methamphetamine (see Table 1.3) (Han \& Gu, 2006; Rothman \& Baumann, 2003).

\begin{tabular}{|l|r|r|r|}
\hline Drug & SERT Affinity & NET Affinity & DAT Affinity \\
\hline MDMA & $238 \pm 13$ & $462 \pm 18$ & $1572 \pm 59$ \\
\hline Cocaine & $304 \pm 10^{2}$ & $779 \pm 30$ & $478 \pm 25$ \\
\hline Methamphetamine & $2137 \pm 98$ & $48 \pm 5.1$ & $114 \pm 11$ \\
\hline
\end{tabular}

Table 1.3: Relative drug affinity for monoamine transporters given as $K_{i}$ (inhibition constant in $\mathrm{nM}$ ) where lower values reflect greater inhibition.

\begin{tabular}{|l|r|r|r|}
\hline Drug & SERT Reversal & NET Reversal & DAT Reversal \\
\hline MDMA & $56.6 \pm 2.1$ & $77.4 \pm 3.4$ & $1572 \pm 59$ \\
\hline Cocaine & $>10,000$ & $>10,000$ & $>10,000$ \\
\hline Methamphetamine & $736 \pm$ & $12.3 \pm 0.7$ & $24.5 \pm 2.1$ \\
\hline
\end{tabular}

Table 1.4: Relative reverse transport for monoamine transporters given as $\mathrm{CG}_{50}$ (intercellular adhesion molecule-3 level in $\mathrm{nM}$ ) where lower values reflect greater neurotransmitter release.

Additionally, SERT blockade and reverse transport of MDMA also disrupt the synthesis of serotonin by inhibiting tryptophan hydroxylase (a crucial player in serotonin synthesis) (see Table 1.4).

\subsection{2 $(5 \alpha, 6 \alpha)-7,8-D i d e h y d r o-4,5 e p o x y-17-m e t h y l m o r p h i n a n-$ 3,6-diol diacetate (ester), Heroin, diamorphine}

Heroin is a powerful narcotic, inducing drowsiness when consumed via ingestion, smoking or injection. Along with morphine, codeine, oxycodone and hydrocodone heroin is a member of the opioid family. Opium comes 
from the poppy plant, Papaver somniferum, and has been used for centuries to alleviate pain. In the early 1800s morphine was isolated and was generously prescribed for the treatment a myriad of health maladies. Widespread use of morphine during the nineteenth century led to a growing concern regarding its abuse liability. Then in 1870 the hypodermic needle was introduced and initially was perceived as a boon in the medical community because it allowed heightened precision of morphine administration. Interestingly, just four years later heroin was synthesised from morphine and its name was coined soon after. It was heralded as a safe, non-addicting, alternative to morphine. This along with a variety of factors, including ease of access, over prescription, and injection of opioids, led to an increase in heroin use, abuse and subsequent addiction. Finally, in 1970, under the Controlled Substances Act, heroin was added to the list of Schedule 1 substances due to its high abuse liability, lack of official medical utility and safety (Dhawan et al., 1996; Hughes, Barker, Crawford, \& Jaffe, 1972; Musto, 1991; Strang, Griffiths, \& Gossop, 1997).

\section{Acute Effects of Heroin}

Intravenous injection is a common method of heroin administration although it can be preceded by subcutaneous injection or uptake through the nasal mucosa. Upon injection heroin is quickly taken up into the brain where it is perceived as a "rush-flash". Heroin leads to the depression of overall central nervous system function and is perceived as a feeling of warmth, drowsiness and euphoria. Dangerously, higher doses of heroin can depress respiration, heart rate and blood pressure to very low levels. Additionally, the individual may feel abdominal contractions caused by spasms between the stomach and small intestine; however, the cough reflex in inhibited and can lead to aspiration into the lungs if the individual vomits. Other acute affects coinciding with heroin administration include injection site vessel and tissue damage, injuries to skeletal muscles that can occur if the individual is unconscious for long periods of time as well as 
constipation (Kendall \& Latter, 2003; Louria, Hensle, \& Rose, 1967; Rook, Huitema, van den Brink, van Ree, \& Beijnen, 2006).

\section{Chronic Effects of Heroin}

The continued use of heroin can quickly lead to tolerance, that is a larger amount of the drug is needed to regain a previously experienced "high". Additionally, due to the prohibitive cost of heroin, if the individual is not able to acquire the substance he/she will begin to experience a series of withdrawal symptoms. Some of these include feelings of anxiety and irritability in addition to a myriad of somatic symptoms: sweating, muscle aches, fever chills, vomiting, excessive tear production and nasal discharge. Increased heart rate, respiration and body temperature also characterise opioid withdrawal (Louria et al., 1967; Musto, 1991).

It has been proposed that the anhedonia described above is related to decreased mesolimbic dopamine transmission when there is a gap in drug taking. Consequently, in an attempt to avoid these negative consequences, individuals may then relapse and begin taking the drug to regain previously enhanced dopamine neurotransmission (De Vries \& Shippenberg, 2002). A study was undertaken to evaluate dopaminergic and serotonergic markers in human heroin addicts; compared to age matched controls addicts had slightly lower dopaminergic activity. This suggests that increased levels of dopamine neurotransmission due to heroin intake may lead to a down regulation of dopamine, as well as its metabolites and transporter. No differences were detected between controls and addicts when serotonin levels were evaluated (Kish et al., 2001).

Several research groups have investigated the neural mechanisms that underlie the changes that occur with prolonged opioid use. For example, like humans, rats show a suite of withdrawal symptoms upon cessation of heroin intake; these include weight loss, writhing, paw tremor and teeth chattering (Dai, Corrigall, Coen, \& Kalant, 1989; Doherty \& Frantz, 2013). Other labs have elucidated the specific areas in the brain that change dur- 
ing opioid administration. Nestler et al. (1996) evaluated the role of the locus coeruleus in opioid withdrawal; chronic opioid administration inhibits activity in this brain structure and over time it develops tolerance to the effects of the drug. Then when the drug is removed activity in the locus coeruleus greatly increases beyond baseline measures suggesting this disrupted activation drives opioid withdrawal; this effect was replicated by the same group in subsequent years (Nestler, 1996, 2004).

Additionally, specific neuroadaptations have been cited as the underlying cause to explain why changes in the brain after chronic opioid administration are long lasting. Jacobs et al. (2002) evaluated long term gene expression in two groups of rats: one had been given heroin passively and the other had self-administered heroin. The animals that were given heroin passively did not show any changes in long term gene expression. Conversely, the animals that had self-administered heroin showed a significant decrease in nucleus accumbens long term gene expression suggesting that the repeated behaviour of self-administration is necessary to initiate changes in gene expression (Jacobs et al., 2002).

Taken together these studies highlight the consequences of chronic heroin intake. Animal studies have provided a useful way to evaluate and understand the chronic effects of heroin. Delineating underlying neural mechanisms may provide the foundation for developing curative treatments for humans.

\section{Heroin Mechanism of Action}

While heroin has a very similar chemical structure to morphine it has higher lipid solubility and this allows for more efficient, that is faster, transmission through the blood brain barrier. After entering the brain heroin is then converted into a metabolite, 6-monoacetylmorphine, and then into morphine (Oldendor, Oldendor, Braun, \& Hyman, 1972; Pardridge, 2012). It is morphine that then binds to three types of opioid peptide receptors: mu, delta and kappa (Dhawan et al., 1996). Upon binding to 
mu-opioid receptors morphine triggers a series of events: first, inhibitory interneurons that influence dopaminergic activity in the mesolimbic "reward" pathway (ventral tegmental area) become hyperpolarised. Second, hyperpolarised interneurons do not provide inhibition to dopamine neurons and this leads to increased dopaminergic activity (Johnson \& North, 1992; Kreek et al., 2012; Nestler, 2004).

It is important to remember that while all drugs of abuse influence the release of dopamine the mechanisms of action by which they do this vary greatly between classes of drugs. As outlined in the previous sections MDMA acts on the serotonin, dopamine and norepinephrine transporters having the greatest impact on the SERT. Conversely, heroin acts indirectly to release dopamine through opioid receptors and interneurons that influence the dopaminergic system.

\subsection{Molecular Analysis}

Drug addiction, or substance use disorder, is technically defined in terms of behavioural changes in the individual. However, there is a large body of research to support the idea that drug addiction facilitates long term changes in the way the brain functions. As discussed earlier researchers have hypothesised that drug addiction represents changes in synaptic plasticity (i.e. learning); where neural connections in the dopaminergic system become inflexible leading to habitual drug intake.

While synaptic plasticity encompasses a variety of processes in different structures throughout the brain a family of proteins, neurotrophic factors, play an important role in synaptic plasticity that underlies learning and memory (Binder \& Scharfman, 2004). 


\subsubsection{Brain Derived Neurotrophic Factor (BDNF)}

One protein of interest is brain derived neurotrophic factor (BDNF); as a member of the neurotrophic factor family it influences the development of the brain by promoting survival and growth of neurons. Additionally, BDNF has been implicated in synaptic transmission, neurogenesis and importantly, the synaptic plasticity that coincides with learning (Binder \& Scharfman, 2004; Conner, Lauterborn, Yan, Gall, \& Varon, 1997). Found widely throughout the central nervous system BDNF binds to the tropomyosin related kinase $\mathrm{B}$ receptor (trkB).

\subsubsection{BDNF and Neuropsychiatric Illness}

BDNF plays a crucial role in neurological basis for psychiatric illness like depression. Research has demonstrated that decreased BDNF levels coincide with depression and stress. However, antidepressants have been shown to be effective in eliminating these effects; over a course of treatment BDNF levels return to normal. While many current antidepressants work by inhibiting reuptake of serotonin this research suggests that regulating levels of BDNF is also important for the treatment of depression (Duman \& Monteggia, 2006; Kozisek, Middlemas, \& Bylund, 2008).

Whereas decreased levels of BDNF contribute to the neurological changes associated with depression there is evidence to suggest that increased BDNF may linked to drug addiction. For example, several research groups have demonstrated that BDNF messenger RNA (mRNA) levels in rats were increased in several brain structures (e.g. striatum, nucleus accumbens) after a single injection of cocaine (Berglind et al., 2007; Le Foll, Diaz, \& Sokoloff, 2005; Liu et al., 2006). Additional research has demonstrated that when BDNF levels were increased in rats so did their self-administration of cocaine (Graham et al., 2007; McGinty, Whitfield, \& Berglind, 2010). Interestingly, in both human and rodent studies researchers have determined that a reduction in the SERT may influence baseline mRNA levels of 
specific coding regions (isoforms) in the BDNF gene. Specifically, mRNA levels of isoforms III, IV, VI and IXa were decreased in rats with a reduction in the SERT (Molteni et al., 2010). The same group then investigated the effects of the antidepressant, duloxetine, which targets the SERT and the norepinephrine transporter (NET) prolonging 5-HT and norepinephrine action in the synapse. After treating HOM and WT animals they found that mRNA levels for total BDNF were increased in the hippocampus, the prefrontal cortex and the frontal cortex. They also investigated changes in specific BDNF isoforms and found that BDNF III mRNA levels were increased in the hippocampus in both genotypes. Interestingly, they also saw increased mRNA levels for BDNF transcripts IV and VI in the HOM animals but not the WT (Calabrese et al., 2010). In addition, studies have shown that treatment with cocaine can lead to differential mRNA level changes across isoforms. For example, Liu et al. (2006) demonstrated that mRNA levels of BDNF isoform IV were significantly increased in the striatum after cocaine administration. Similarly, research has shown that cocaine exposure facilitates brain region specific increases in mRNA levels of BDNF isoforms (e.g. increased BDNF isoform IV in the medial prefrontal cortex) (Sadri-Vakili et al., 2010; H. D. Schmidt et al., 2012). These studies suggest a specific role for BDNF in the neurological changes that accompany drug addiction. Therefore, investigating changes in BDNF will add valuable insight into the specific drug induced neuroadaptations that occur in individuals with altered serotonergic function.

\subsection{Brief Outline of Behavioural and Molecular Experiments}

The suite of current experiments seeks to elucidate the complex interaction between genetic risk, a reduction in the serotonin transporter, and the development of drug addiction. In other words: Is the serotonin trans- 


\subsection{BRIEF OUTLINE OF BEHAVIOURAL AND MOLECULAR EXPERIMENTS}

porter a risk factor in the development of drug addiction? To investigate this question in the laboratory the first two experiments evaluated the behaviour of SERT ${ }^{-/-}(\mathrm{HOM}), \mathrm{SERT}^{+/-}(\mathrm{HET})$, and SERT ${ }^{+/+}(\mathrm{WT})$ rats when completing drug self-administration with MDMA and with heroin. The drug self-administration behavioural paradigm has become the accepted "gold standard" for studying drug addiction pre-clinically and has many advantages. Some of these include the ability to monitor the exact amount of drug intake as animals are implanted with indwelling catheters for drug delivery. Additionally, drug self-administration allows the investigator to scrutinise various stages of drug taking behaviour (e.g. maintenance responding shows how animals regulate drug intake to maintain a specific level of drug consumption or responding on a progressive ratio schedule can demonstrate motivation to pursue additional infusions of drug). In modelling various aspects of drug taking behaviour it is a powerful tool not only for understanding drug addiction but for screening potential pharmacological treatments (Panlilio \& Goldberg, 2007; Richardson \& Roberts, 1996).

Because drug addiction is often comorbid with other psychiatric illnesses like anxiety all animals completed a novelty suppressed feeding paradigm before they underwent surgery for the drug self-administration experiments. This test measured latency to begin eating a food pellet set in the middle of an open field apparatus where longer latency corresponds with a higher level of anxiety. Drug self-administration behaviour was then evaluated against latency to begin feeding to determine if a correlation existed (Lira et al., 2003; Olivier et al., 2008).

Following the drug self-administration experiments a set of locomotor activity experiments were completed. Groups of homozygous (SERT ${ }^{-1-}$, $\mathrm{HOM}$ ), heterozygous (SERT $\left.{ }^{+/}, \mathrm{HET}\right)$, and wild type $\left(\mathrm{SERT}^{+/+}, \mathrm{WT}\right)$ were treated with MDMA (or vehicle) and ambulatory counts were measured. A second identical experiment was conducted with heroin. These experiments provided an opportunity to evaluate the acute effects of multiple 
doses of MDMA and heroin on locomotion. Additionally, locomotor activity has been linked to the rewarding properties of drugs of abuse; that is, dopaminergic transmission within the mesolimbic system has been implicated as a mechanism for both rewarding drug effects and locomotion (Beninger, 1983; Pijnenburg \& van Rossum, 1973). Therefore, these experiments served to identify any differences between the genotypes for both drugs.

Lastly, to complement the locomotor activity experiments, the potentially negative consequences of MDMA and heroin were evaluated with two conditioned taste aversion experiments. Conditioned taste aversion is elicited when a drug, in this case either MDMA or heroin, is paired with a novel sucrose solution; when given a choice between sucrose and water animals will cease to drink the sucrose. This reduced intake suggests that the sucrose solution has been devalued as a reinforcer and consequently the animal will choose to drink water (Goudie, 1979; Hunt \& Amit, 1987). Therefore, these experiments served to identify any differences between the genotypes for both drugs.

Finally, in addition to the behavioural experiments outlined above the striatal brain region of the animals that completed the drug self-administration experiments was analysed with quantitative reverse transcription polymerase chain reaction (qRT-PCR). This technique has become widely used since its advent 32 years ago and is a relatively straightforward way to evaluate changes in gene expression. It also allows for a more in depth understanding of the molecular changes that accompany drug addiction and serves as a useful complement to the behavioural changes investigated in the drug self-administration experiments described earlier (Bustin, Benes, Nolan, \& Pfaffl, 2005; VanGuilder, Vrana, \& Freeman, 2008).

Overall, it is predicted that a reduction in the serotonin transporter will confer susceptibility to the development of drug addiction as measured by the suite of experiments described above. Specific predictions for each experiment are given in the following chapters. 


\section{Chapter 2}

\section{Novelty Suppressed Feeding and Drug Self-Administration}

\subsection{Introduction}

Addiction to drugs of abuse is a ubiquitous, worldwide, phenomenon that places a tremendous financial and psychological burden on society, families and the individual. The DSM-5 defines drug addiction as a series of behavioural changes: that is, over time individuals have difficulty in limiting their drug use, they are extremely motivated to obtain the drug and are willing to pursue it even despite negative consequences (American Psychiatric Association, 2013). Currently, only about 1 in 6 "addicts" seek help in the clinic and, sadly, many do not achieve a successful outcome from their treatment regimen. This fact has led research groups to continue studying the complex interplay of behavioural and neurochemical changes that accompany drug addiction in an effort to better inform treatment programs for humans (United Nations Office on Drugs and Crime, 2014).

Interestingly, only a small percentage of individuals ( 20\%) who use drugs of abuse actually go on to develop the compulsive behaviours that define addiction (Deroche-Gamonet \& Piazza, 2010). Clinical studies have 
shown that there is a subset of the population with a genetically determined reduction in the serotonin transporter protein (SERT) that may increase vulnerability for developing psychiatric disorders like anxiety, depression and drug addiction (Lesch et al., 1996; Lesch \& Gutknecht, 2005; Murphy et al., 2001). It should be noted that the polymorphism in the promotor region of the SLC64A gene is not the only molecular alteration implicated in the development of drug addiction. However, due to the relatively common incidence of this polymorphism and its relationship to other psychiatric illnesses it may represent an important avenue for understanding why some individuals develop addiction to drugs of abuse while others do not.

Therefore, to systematically investigate the influence of reduced serotonin transporter function in the laboratory we studied the behaviour of a genetically altered animal model: the serotonin transporter (SERT) knockout rat. Homozygous (SERT ${ }^{-/}$, HOM) animals lack SERT function completely while heterozygous (SERT ${ }^{+/}$, HET) have about 50\% SERT function compared to the wild type (SERT ${ }^{+/+}$,WT) (Smits et al., 2006).

It is important to note that all drugs of abuse lead to the stimulation of dopamine release and this is thought to underlie the initial rewarding effects of drugs of abuse (Everitt et al., 2008; Koob et al., 1998; Koob \& Volkow, 2010; Nestler, 1996, 2001). However, the precise mechanism of action varies widely between different classes of drugs. For example, while MDMA, a psychostimulant, directly stimulates the release of dopamine its primary action targets the serotonin transporter (Green et al., 2003) (Han \& Gu, 2006; Rothman \& Baumann, 2003). Studies investigating this complex mechanism of action have shown that MDMA induced 5-HT release may inhibit dopamine neurotransmission (Alex \& Pehek, 2007; Rothman \& Baumann, 2006). Unlike MDMA, the opioid heroin, stimulates indirect dopamine release via action on the $\mu$-opioid receptor (Johnson \& North, 1992; Kreek et al., 2012; Nestler, 2004). Taken together it is possible that reduced SERT function and consequently reduced 5-HT release, may lead 
to a sensitised dopaminergic response to drugs of abuse.

To understand the interaction between reduced SERT function and the development of addictive behaviours groups of HOM, HET and WT rats completed a drug self-administration behavioural paradigm. Drug selfadministration is regarded as the "gold standard" for evaluating drug addiction in the laboratory as it allows for precise measurement of how much drug is taken. Additionally, it mirrors some of the hallmarks of drug addiction as outlined in the DMS-5; for example, you can evaluate how motivated an animal is to receive a drug infusion by requiring additional "work" (lever presses) in order to earn it (Panlilio \& Goldberg, 2007; Richardson \& Roberts, 1996).

Drug self-administration behaviour was evaluated in animals taking either MDMA or heroin to determine if disrupted serotonergic transmission interacts with the class of drug being self-administered. Prior to this suite of experiments another research group had repeatedly demonstrated that in rats with normal SERT function only about $50 \%$ of those animals acquire MDMA self-administration (Schenk, 2009, 2011; Schenk, ColussiMas, Do, \& Bird, 2012). This result is in line with the idea that MDMA induced 5-HT release may produce inhibition on dopamine neurotransmission and subsequently lead to reduced MDMA self-administration. It is important to note that these experiments were carried out in SpragueDawley (SD) rats; however, the SERT KO rats have a Wistar background. Therefore, it was of interest to determine if SERT WT animals, with intact SERT function, would respond similarly to the SD animals that had self-administered MDMA.

Lastly, since a reduction in the SERT has been implicated in a variety of psychiatric illnesses, like anxiety and mood disorders, and these are often comorbid with drug addiction, it was important to evaluate levels of anxiety before drug self-administration (Lesch et al., 1996; Lesch \& Gutknecht, 2005; Murphy et al., 2001). Therefore, all animals completed a novelty suppressed feeding paradigm to measure anxiety. Any genotype differ- 
ences could then be compared to the subsequent drug self-administration behaviour.

\subsubsection{Predictions}

Prediction 1: In the novelty suppressed feeding paradigm higher levels of anxiety will be inversely related to SERT function (HOM > $\mathrm{HET}>\mathrm{WT})$.

Prediction 2: As MDMA targets the SERT and because 5-HT release may inhibit dopamine neurotransmission, it is predicted that MDMA self-administration will be inversely related to SERT function (HOM $>$ HET $>$ WT).

Prediction 3: As heroin, like MDMA, stimulates dopamine release (albeit indirectly) it is predicted that heroin self-administration will be inversely related to SERT function $(\mathrm{HOM}>\mathrm{HET}>\mathrm{WT})$.

\subsection{Methods}

\subsubsection{Novelty Suppressed Feeding}

\section{Subjects}

All experimental procedures were approved by the Animal Ethics Committee at Victoria University of Wellington. Subjects were male Wistar rats (HOM, HET and WT). Breeding occurred in the vivarium at Victoria University of Wellington and animals were housed in groups until they underwent novelty suppressed feeding testing. Before testing animals were isolated and housed individually in a temperature $\left(19-21^{\circ} \mathrm{C}\right)$ and humidity (55\%) controlled room that was maintained on a 12 hour light-dark phase with lights on at 0700 . Testing occurred during the light phase beginning 
each day at 0800 . Water was available ad libitum but food availability was restricted as described in the procedure below.

\section{Apparatus}

The novelty suppressed feeding experimental procedure was conducted as previously described (Lira et al., 2003; Olivier et al., 2008). Subjects were food deprived for 24 hours prior to testing. Testing began one hour after subjects had been transferred to the experimental room in their home cages. Then, under dim light, animals were observed individually in a circular open field apparatus. Clean bedding covered the floor of the open field and was replaced between subjects. A single food pellet was placed on a small piece of circular paper $(6.25 \mathrm{~cm})$ in the centre of the open field.

\section{Testing Schedule}

Testing commenced when subjects were placed in a preselected "start" location, adjacent to the wall, in the open field. Latency to begin feeding was recorded with a maximum value of $10 \mathrm{~min}$ (600 seconds). After a subject began feeding or $10 \mathrm{~min}$ had elapsed the subject was removed from the open field and placed back into its home cage. All subjects were returned to the housing room after the conclusion of the experiment and given free access to food.

\subsubsection{Drug Self-Administration}

\section{Subjects}

After the completion of novelty suppressed feeding testing subjects remained individually housed and underwent the surgical procedure described below. Subjects weighed $280-380 \mathrm{~g}$ at the time of surgery. Testing occurred during the light phase beginning each day at 0700 when animals 
were transferred from their home cages to the experimental room. Food and water was available ad libitum except during testing.

\section{Surgery}

Groups of 13-18 animals, per genotype, underwent surgery. Subjects were anesthetised with ketamine $\left(90 \mathrm{mg} \mathrm{kg}^{-1}\right)$ and xylazine $\left(9 \mathrm{mg} \mathrm{kg}^{-1}\right)$ via an intraperitoneal (i.p.) injection and an indwelling catheter was implanted in the right jugular vein. The analgesic carprofen $\left(5.0 \mathrm{mg} \mathrm{kg}^{-1}\right)$ was administered via subcutaneous (s.c.) injection immediately before surgery and the subjects eyes were swabbed with Lacrilube. The area over the right jugular vein was shaved and cleansed with ethanol and iodine. Through an incision in the upper right chest quadrant the jugular vein was isolated and tied off. A length of tubing was inserted through a small cut made in the vein. The distal portion of the tubing was passed subcutaneously to an exposed portion of the skull where it was connected to a 2 $\mathrm{cm}$ length of 22 ga stainless steel tubing. The tubing was then secured to the skull with four jeweller's screws and acrylic dental paste. All incisions were treated topically with Terramycin and electrolytes were administered (s.c.). Postoperatively, subjects were treated with carprofen $\left(5.0 \mathrm{mg} \mathrm{kg}^{-1}\right)$ (s.c.) for two days and their catheters were flushed daily with $0.2 \mathrm{~mL}$ of a sterile solution containing heparin $\left(30 \mathrm{IU} \mathrm{mL} \mathrm{mL}^{-1}\right)$ and penicillin $\mathrm{G}$ potassium $\left(250,000 \mathrm{IU} \mathrm{mL} \mathrm{mL}^{-1}\right)$ to prevent infection and the formation of clots. Subjects recovered for 5-7 days after surgery before they commenced drug self-administration testing.

\section{Apparatus}

Before each session rats were weighed and flushed with $0.2 \mathrm{~mL}$ of a sterile solution containing heparin $\left(30 \mathrm{IU} \mathrm{mL}^{-1}\right)$ and penicillin $\mathrm{G}$ potassium (250,000 IU mL $\left.{ }^{-1}\right)$ before being transported to the testing room. Drug selfadministration was carried out in Med Associates test chambers that were 
housed in sound attenuating boxes. The testing room was temperature $\left(19-21^{\circ} \mathrm{C}\right)$ and humidity (55\%) controlled. Each box was equipped with two levers; depression of the active lever delivered an infusion of the drug while depression of the inactive lever was counted but produced no other consequence. During each drug infusion $0.1 \mathrm{~mL}$ solution (MDMA) was delivered to the subject over 12 seconds via a Razel pump equipped with a $20 \mathrm{~mL}$ syringe. With each infusion a light located above the active lever was illuminated (for the duration of the infusion). Each training session began when the subject was placed in the chamber, connected to the line and the experimenter delivered a single infusion (primer, uncounted, light illuminated) of the drug.

A slightly modified procedure was used for heroin self-administration. Upon depression of the active lever $0.025 \mathrm{~mL}$ of heroin drug solution was delivered to the subject over 3 seconds via a Razel pump equipped with a $20 \mathrm{~mL}$ syringe. The smaller volume, $0.025 \mathrm{~mL}$, was used to ensure that animals were not taking drug too quickly. In addition, a 30 second time out was added after each infusion where no infusion could be earned despite presses on the active lever. These procedures are common practice with narcotic drugs like heroin (Dai et al., 1989; Doherty \& Frantz, 2012).

\section{Testing Schedule}

For MDMA self-administration subjects were placed in the testing chambers on an FR1 (fixed ratio; where 1 active lever press delivers 1 infusion) schedule for daily two hour self-administration sessions $\left(1.0 \mathrm{mg} \mathrm{kg}^{-1}\right.$ per infusion MDMA). Acquisition of MDMA self-administration occurred after 85-90 total presses on the active lever within 25 test sessions. When subjects met this criterion the dose was then halved $\left(0.5 \mathrm{mg} \mathrm{kg}^{-1}\right.$ per infusion MDMA). After responding had stabilised animals were placed on an FR2 (fixed ratio; where 2 active lever presses deliver 1 infusion) schedule and finally on an FR5 schedule. Stable responding was defined as three consecutive days where total active lever presses varied less than $20 \%$. Next, 
subjects underwent progressive ratio testing where the number of lever presses for each subsequent infusion increased systematically (i.e. 1, 2, 4, 6, 9, 12 etc.) (Richardson \& Roberts, 1996). Progressive ratio testing was completed for three counterbalanced doses of MDMA: $0.25 \mathrm{mg} \mathrm{kg}^{-1}$ per infusion, $0.5 \mathrm{mg} \mathrm{kg}^{-1}$ per infusion and $1.0 \mathrm{mg} \mathrm{kg}^{-1}$ per infusion. Between progressive ratio doses subjects were placed back on FR5 until responding returned to their previous level of FR5 responding (at least one session was completed). After the last progressive ratio dose animals completed one additional day on the FR5 schedule to resume normal responding. The next day subjects were euthanised via $\mathrm{CO}_{2}$ asphyxiation and brains were extracted and frozen immediately at $-80^{\circ} \mathrm{C}$.

For heroin self-administration subjects were placed in the testing chambers on an FR1 (fixed ratio; where 1 active lever press delivers 1 infusion) schedule for daily two hour self-administration sessions $\left(0.05 \mathrm{mg} \mathrm{kg}^{-1}\right.$ per infusion heroin). Subjects completed 15 self-administration sessions; then the dose was doubled $\left(0.1 \mathrm{mg} \mathrm{kg}^{-1}\right.$ per infusion heroin) until responding was stable (at least three consecutive days). When subjects met this criterion they were placed on an FR2 (fixed ratio; where 2 active lever presses deliver 1 infusion) schedule and finally on an FR5 schedule. Next, subjects underwent progressive ratio testing where the number of presses for each subsequent infusion increased systematically (i.e. 1, 2, 4, 6, 9, 12 etc.) (Richardson \& Roberts, 1996). This schedule was slightly modified: four breakpoints $(2,4,9$ and 12) at the beginning of the schedule were eliminated to decrease over-sedation at the start of the session (Smith \& Aston-Jones, 2012). Progressive ratio testing was completed for three doses of heroin: $0.05 \mathrm{mg} \mathrm{kg}^{-1}$ per infusion, $0.1 \mathrm{mg} \mathrm{kg}^{-1}$ per infusion and $0.2 \mathrm{mg} \mathrm{kg}^{-1}$ per infusion. After the last progressive ratio dose animals completed one additional day on the FR5 schedule to resume normal responding. The next day subjects were euthanised via $\mathrm{CO}_{2}$ asphyxiation and brains were extracted and frozen immediately at $-80{ }^{\circ} \mathrm{C}$. 


\section{Drugs}

Drug solutions for infusion were prepared by mixing 3,4-methylenedioxymethamphetamine (MDMA) or Heroin $\mathrm{HCl}$ (BDG Synthesis, Wellington, New Zealand), in powder form, with heparinised saline. Before use intravenous drug solutions were clarified of impurities using hypophilic Minisart syringe filters and placed directly into sterile $20 \mathrm{~mL}$ syringes. Syringes were labelled with the type of drug and corresponding "weight class". Weight classes were calculated to account for rats of differing weights; it was not uncommon for rats to weigh $280-400 \mathrm{~g}$ (with rats gaining weight, up to $400 \mathrm{~g}$, throughout the experiment). Therefore, rats were divided into three weight classes: featherweight $300 \mathrm{~g}$ (280-320 g), welterweight $340 \mathrm{~g}$ (320-360 g), and heavyweight $380 \mathrm{~g}$ (360-400 g) with drug solutions prepared according to these weights. When an animal reached a boundary between two weight classes he would start the following testing day with the solution for the next weight class.

\subsection{Results}

\subsubsection{MDMA}

\section{Novelty Suppressed Feeding}

Before drug self-administration surgery and testing was conducted subjects completed a novelty suppressed feeding paradigm. Latency to begin feeding was recorded and compared across groups of HOM, HET and WT subjects. A one-way ANOVA revealed no significant difference between the genotypes, $F(2,47)=.62, p=.55$ (see Figure 2.1).

\section{Drug Self-Administration}

After recovery from surgery subjects began MDMA self-administration testing. Due to a small number of unsuccessful surgeries or early loss of 


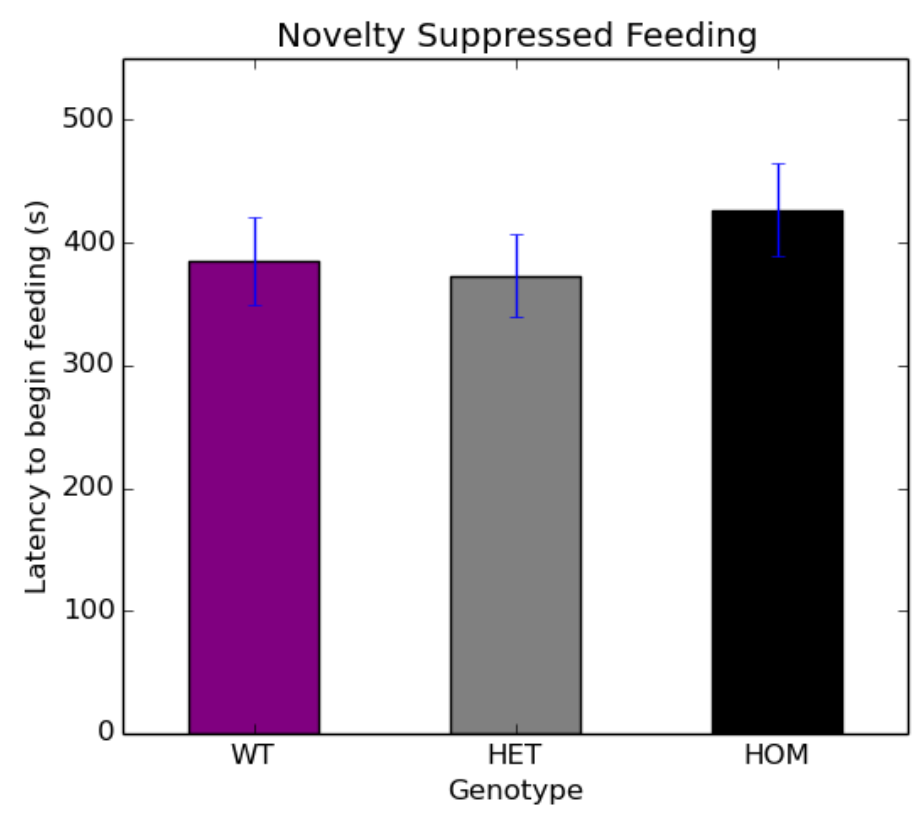

Figure 2.1: Average (+SEM) latency to begin feeding for HOM ( $\mathrm{n}=16)$, HET $(n=18)$ and WT $(n=16)$ subjects.

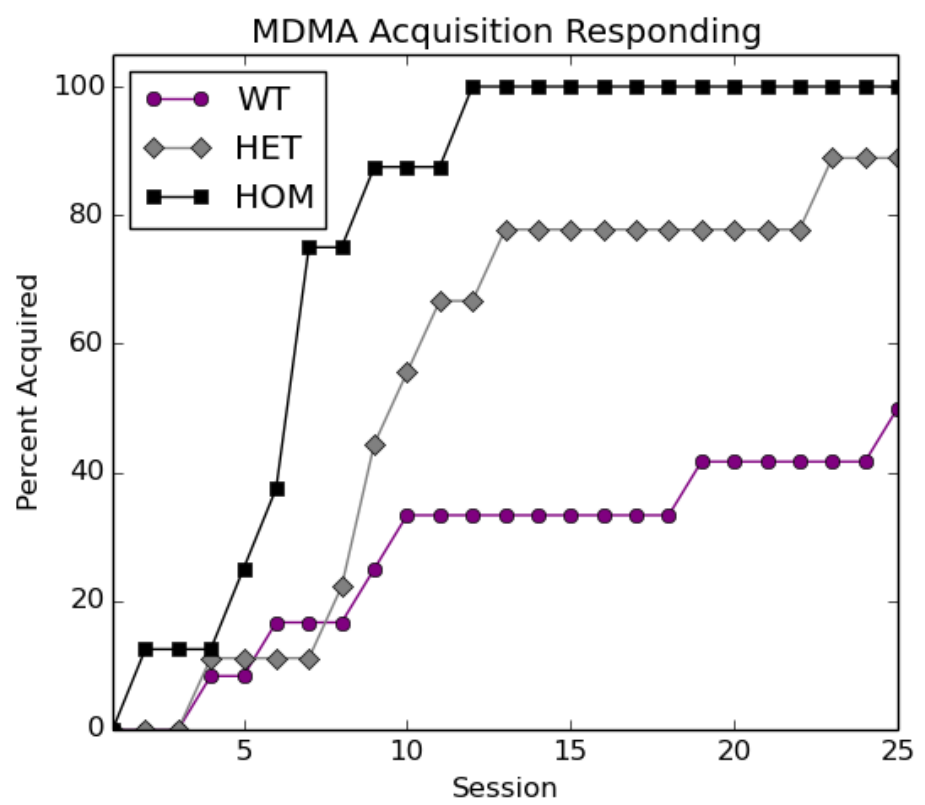

Figure 2.2: Acquisition responding for MDMA self-administration for $\operatorname{HOM}(n=8), \operatorname{HET}(n=9)$ and WT $(n=12)$ groups. 
catheter patency while subjects were recovering from surgery the group sizes were slightly reduced from those described for novelty suppressed feeding. Subjects completed MDMA self-administration until they reached acquisition criteria: 85-90 total presses on the active lever. Upon reaching this criterion they then began the first schedule of Maintenance testing. If subjects did not meet this criterion in the first 25 test sessions they were removed from the study. Kaplan-Meier survival analysis revealed a significant genotype effect $\left(\chi^{2}=13.83, p=.001\right)$. Acquisition of MDMA self-administration is given in Figure 2.2; after 25 test sessions $100 \%$ of HOM subjects had acquired MDMA self-administration with the HET and WT groups reaching $89 \%$ and $50 \%$, respectively. Post hoc comparisons revealed a significant difference between the HOM and both the HET and WT groups; however, the comparison between the HET and WT groups did not reach significance but it may indicate a trend toward an effect (see Table 2.1).

\begin{tabular}{|c|c|}
\hline \multicolumn{2}{|r|}{$\boldsymbol{p}$ value } \\
\hline HOM - HET & $\mathbf{0 . 0 3}^{*}$ \\
\hline HOM - WT & $\mathbf{0 . 0 0 2}^{*}$ \\
\hline HET - WT & 0.06 \\
\hline
\end{tabular}

Table 2.1: Acquisition responding for MDMA self-administration: Post hoc comparisons. *Denotes statistical significance.

The day after reaching acquisition criterion subjects remained on an FR1 schedule but administered a half dose ( $0.5 \mathrm{mg} \mathrm{kg}^{-1}$ per infusion) until responding was stable (at least three consecutive days). Then subjects completed FR2 and FR5 schedules of responding.

A repeated measures ANOVA revealed a significant main effect of genotype $F(2,15)=4.82, p=.02$, and fixed ratio schedule $F(2,30)=8.64, p=.001$ on lever presses. The genotype*fixed ratio schedule interaction did not reach significance but it may indicate a trend toward an effect $F(4,30)=$ 
$2.34, p=.08$.

One-way ANOVA post hoc analysis of FR1 responding demonstrated a significant difference among the genotypes, $F(2,15)=6.87, p=.008$ ) with HOM subjects showing significantly higher responding compared to HET and WT subjects. However, there was no significant difference between HET and WT groups. Similarly, post hoc analysis of FR2 responding showed a significant difference between the genotypes, $F(2,15)$ $=4.06, p=.04$, where HOM subjects had higher responding than HET however both the HOM/WT and HET/WT comparisons failed to reach significance. Lastly, when FR5 responding was analysed differences between the groups did not reach significance but may indicate a trend toward an effect $(p=.07)$ (see Figure 2.3 and Table 2.2).

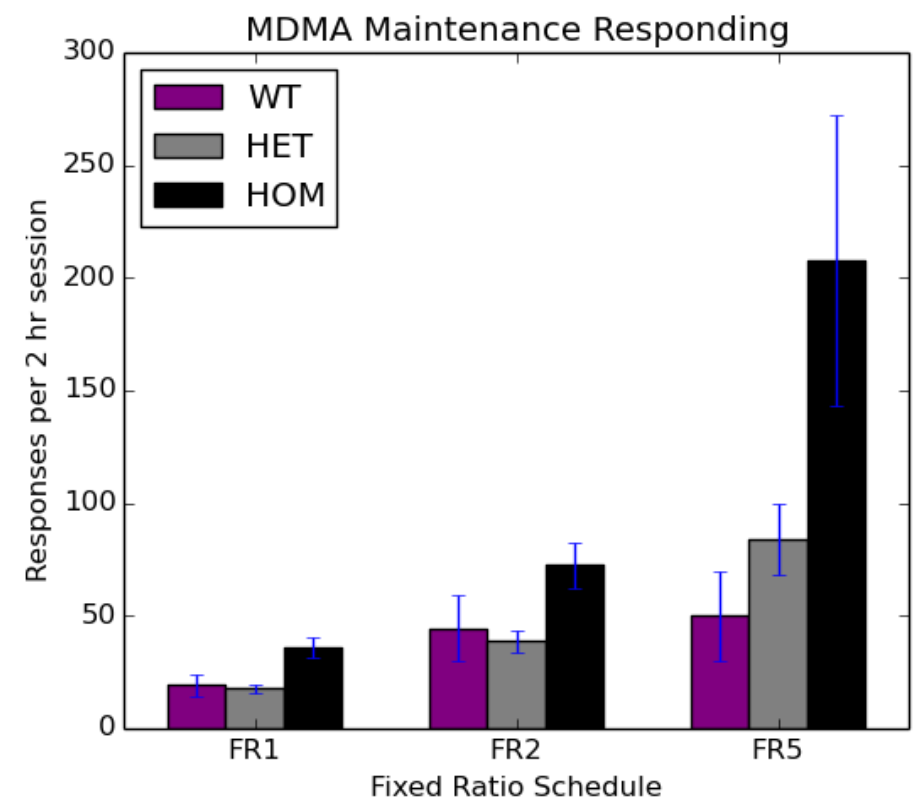

Figure 2.3: Maintenance responding for MDMA self-administration. Average (+SEM) responding across varying fixed ratio schedules for HOM (n $=7)$, HET $(n=7)$ and WT $(n=4)$ subjects.

Upon further investigation within each genotype across the fixed ratio 


\begin{tabular}{|c|c|c|c||l|c|c|c|}
\hline & FR1 & FR2 & FR5 & & HOM & HET & WT \\
\hline HOM - HET & $\mathbf{0 . 0 0 9}^{*}$ & $\mathbf{0 . 0 4}^{*}$ & 0.133 & FR1 - FR2 & 0.78 & 0.29 & 0.47 \\
\hline HOM - WT & $\mathbf{0 . 0 4}^{*}$ & 0.165 & 0.1 & FR2 - FR5 & 0.05 & $\mathbf{0 . 0 1}^{*}$ & 0.96 \\
\hline HET - WT & 0.97 & 0.92 & 0.88 & FR1 - FR5 & $\mathbf{0 . 0 1}^{*}$ & $\mathbf{0 . 0 0 1}^{*}$ & 0.34 \\
\hline
\end{tabular}

Table 2.2: Maintenance responding for MDMA self-administration: Post hoc comparisons. *Denotes statistical significance.

schedules HOM subjects showed a significant difference among fixed ratio schedules, $F(2,18)=5.70, p=.012$, with significantly lower responding on FR1 and FR2 fixed ratio schedules compared to FR5. The FR1/FR2 comparison failed to reach significance. Like the HOM the HET group showed a significant difference between fixed ratio schedules, $F(2,18)=$ $12.67, p<.001$, with significantly lower responding on FR1 and FR2 fixed ratio schedules compared to FR5. The FR1/FR2 comparison failed to reach significance. Finally, the WT group revealed no difference across the fixed ratio schedules, $F(2,9)=1.27, p=.33$ (see Table 2.2).

After maintenance responding subjects completed a progressive ratio schedule with three doses of MDMA: $0.25 \mathrm{mg} \mathrm{kg}^{-1}, 0.5 \mathrm{mg} \mathrm{kg}^{-1}$ and $1.0 \mathrm{mg} \mathrm{kg}^{-1}$. A repeated measures ANOVA revealed a significant main effect of dose, $F(2,20)=6.78, p=.006$, on the number of infusions; however, the main effect of genotype did not reach significance but may indicate a trend toward an effect $F(2,10)=3.62, p=.07)$. The dose ${ }^{*}$ genotype interaction failed to reach significance, $F(4,20)=1.55, p=.23$.

One-way ANOVA post hoc analysis of the $0.25 \mathrm{mg} \mathrm{kg}^{-1}$ and $0.5 \mathrm{mg} \mathrm{kg}^{-1}$ doses revealed no difference between genotypes. However, although an effect among the genotypes when responding on the $1.0 \mathrm{mg} \mathrm{kg}^{-1}$ dose did not reach significance it may indicate a trend toward an effect $F(2,10)=$ 3.46, $p=.07$ (see Figure 2.4 and Table 2.3).

Upon further investigation within each genotype HOM subjects showed a significant difference between the doses, $F(2,6)=4.98, p=.05$. They 


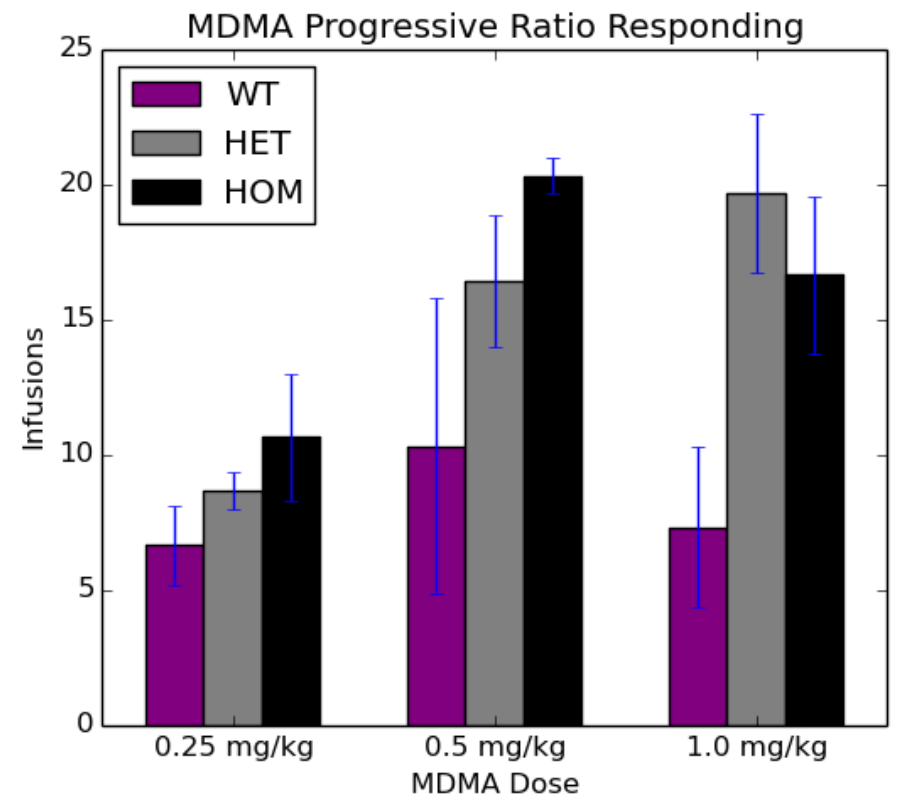

Figure 2.4: Progressive Ratio responding for MDMA self-administration. Average (+SEM) responding across varying MDMA doses on a progressive ratio schedule for $\operatorname{HOM}(n=3)$, HET $(n=7)$ and WT $(n=3)$ subjects.

\begin{tabular}{|c|c|c|c|}
\hline & MDMA 0.25 & MDMA 0.5 & MDMA 1.0 \\
\hline HOM - HET & 0.53 & 0.67 & 0.8 \\
\hline HOM - WT & 0.18 & 0.2 & 0.26 \\
\hline HET - WT & 0.5 & 0.4 & 0.06 \\
\hline \hline & HOM & HET & WT \\
\hline MDMA 0.25 - 0.5 & 0.05 & .06 & 0.77 \\
\hline MDMA 0.25 - 1.0 & 0.21 & $\mathbf{0 . 0 1}$ & 0.99 \\
\hline MDMA 0.5 - 1.0 & 0.5 & 0.56 & 0.84 \\
\hline
\end{tabular}

Table 2.3: Progressive ratio responding for MDMA self-administration: Post hoc comparisons. ${ }^{*}$ Denotes statistical significance. 
demonstrated significantly lower responding for the $0.25 \mathrm{mg} \mathrm{kg}^{-1}$ dose compared to the $0.5 \mathrm{mg} \mathrm{kg}^{-1}$ dose. The $0.25 \mathrm{mg} \mathrm{kg}^{-1} / 1.0 \mathrm{mg} \mathrm{kg}^{-1}$ and 0.5 $\mathrm{mg} \mathrm{kg}^{-1} / 1.0 \mathrm{mg} \mathrm{kg}^{-1}$ comparisons failed to reach significance. Similarly, HET subjects showed a significant difference in progressive ratio responding, $F(2,18)=6.39, p=.008$. They showed significantly lower responding on the $0.25 \mathrm{mg} \mathrm{kg}^{-1}$ dose compared to the $1.0 \mathrm{mg} \mathrm{kg}^{-1}$ dose; a difference between responding on the $0.25 \mathrm{mg} \mathrm{kg}^{-1}$ and $0.5 \mathrm{mg} \mathrm{kg}^{-1}$ doses did not reach significance but may indicate a trend toward an effect. The $0.5 \mathrm{mg} \mathrm{kg}^{-1} / 1.0 \mathrm{mg} \mathrm{kg}^{-1}$ comparison failed to reach significance. Lastly, comparing WT responding across doses revealed no significant difference, $F(2,6)=.28, p=.76$ (see Table 2.3 ). It should be noted that due the lengthy nature of drug self-administration experiments some subjects lost catheter patency before completing the entire experiment; therefore, the sample sizes decreased as the experiment progressed.

\subsubsection{Heroin}

\section{Novelty Suppressed Feeding}

Before drug self-administration surgery and testing was conducted subjects completed a novelty suppressed feeding paradigm. Latency to begin feeding was recorded and compared across groups of HOM, HET and WT subjects. A one-way ANOVA did not reach significance but may indicate a trend toward an effect between the genotypes, $F(2,38)=3.34, p=.05$ (see Figure 2.5). Post hoc analysis did not reach significance but may indicate a trend toward an effect when both the HOM and HET were compared to the WT group. The difference between HOM and HET groups failed to reach significance (see Table 2.4). 


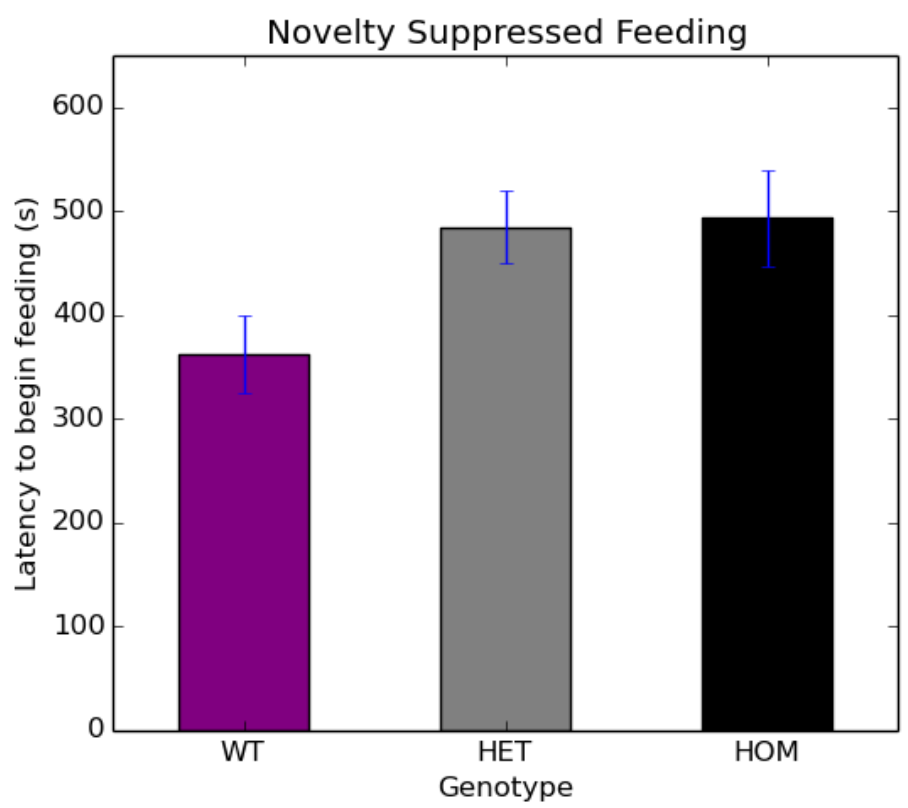

Figure 2.5: Average (+SEM) latency to begin feeding for HOM $(n=13)$, HET $(\mathrm{n}=15)$ and WT $(\mathrm{n}=13)$ subjects.

\begin{tabular}{|c|c|}
\hline & $\boldsymbol{p}$ value \\
\hline HOM - HET & 0.99 \\
\hline HOM - WT & 0.07 \\
\hline HET - WT & 0.08 \\
\hline
\end{tabular}

Table 2.4: Novelty Suppressed Feeding: Post hoc comparisons.

\section{Drug Self-Administration}

After recovery from surgery subjects began heroin self-administration testing. Due to a small number of unsuccessful surgeries or early loss of catheter patency while subjects were recovering from surgery the group sizes were slightly reduced from those described for novelty suppressed feeding. Subjects completed self-administration for 15 days $\left(0.05 \mathrm{mg} \mathrm{kg}^{-1}\right.$ 
per infusion heroin). Upon completing this initial phase they then began the first schedule of Maintenance testing.

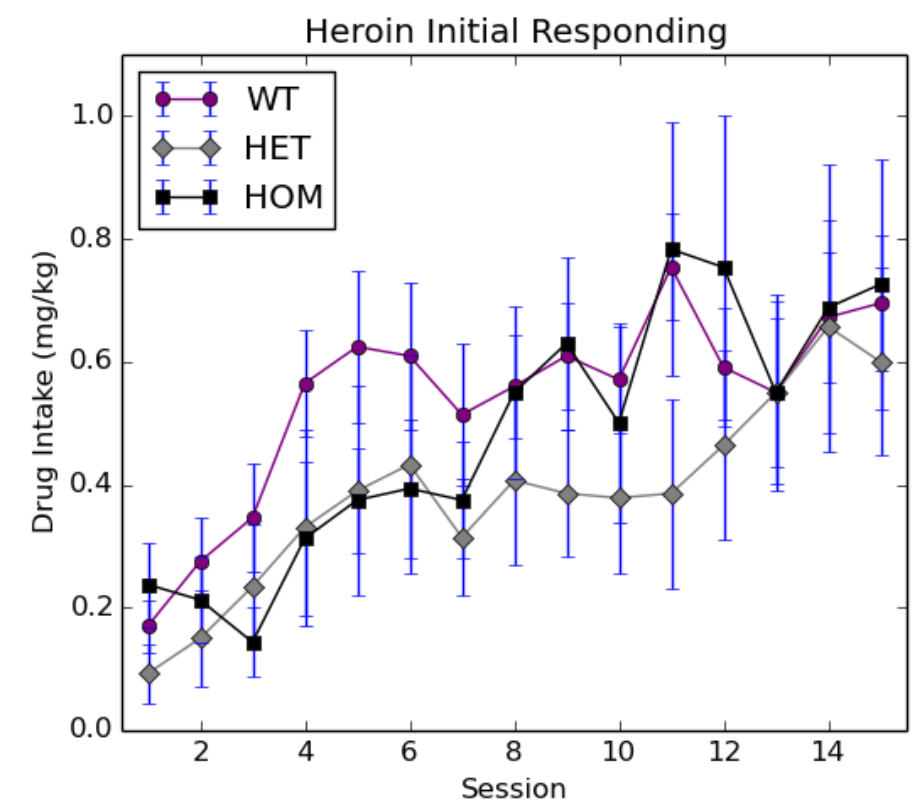

Figure 2.6: Initial drug intake for Heroin self-administration for HOM $(n=8), \operatorname{HET}(n=7)$ and WT $(n=10)$ groups.

When evaluating drug intake over this period a one-way ANOVA revealed no differences between the genotypes $F(2,22)=.645, p=.53$. However, analysis comparing Days 1-7 to Days 8 -15 revealed a significant main effect of day $F(2,22)=36.7, p<.001$, indicating an increase in responding during Days 8-15 compared to Days 1-7 (see Figure 2.6). The main effect of genotype, $F(2,22)=.653, p=.53$, and the days* ${ }^{*}$ enotype interaction, $F(2$, 22) $=1.80, p=.19$, failed to reach significance.

On Day 16 subjects remained on an FR1 schedule but administered a double dose $\left(0.1 \mathrm{mg} \mathrm{kg}^{-1}\right.$ per infusion heroin) until responding was stable (at least three consecutive days). The animals were then moved to FR2 and FR5 (using the same procedure for stable responding).

To compare maintenance responding on fixed ratio schedules, FR1, FR2 


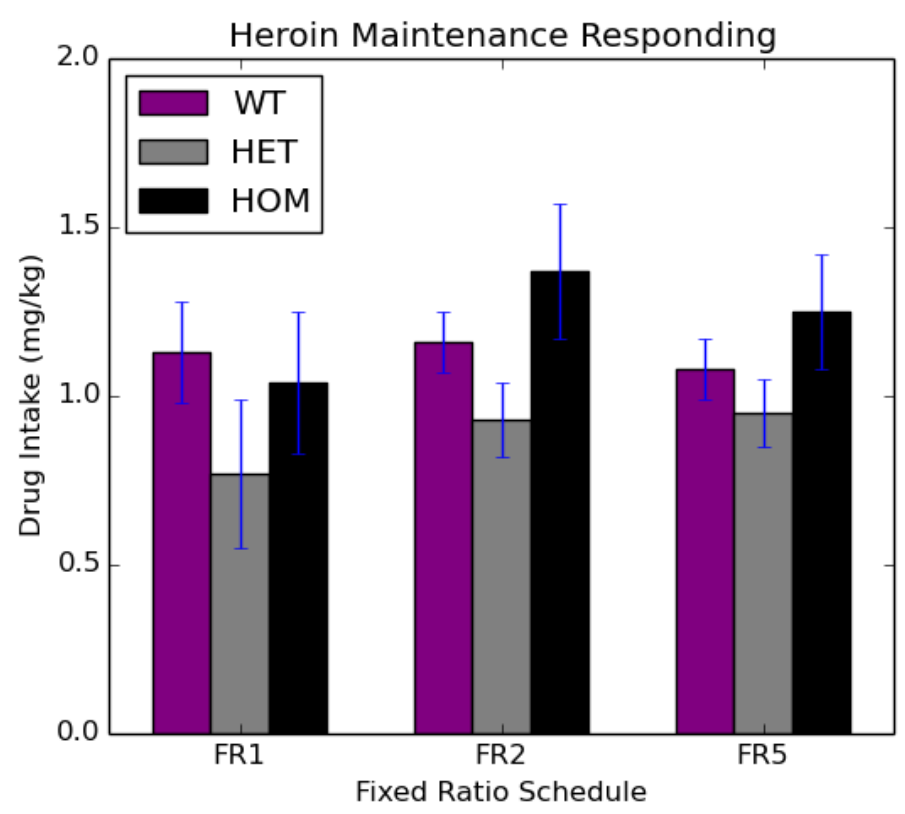

Figure 2.7: Maintenance responding for heroin self-administration across varying fixed ratio schedules for $\operatorname{HOM}(n=6), \operatorname{HET}(n=5)$ and WT $(n=7)$ subjects.

and FR5, a repeated measures ANOVA was conducted but failed to reveal a significant main effect of genotype $F(2,15)=1.026, p=.38$, or fixed ratio schedule $F(2,30)=.952, p=.40$ on lever presses. Additionally, the genotype ${ }^{*}$ fixed ratio schedule interaction failed to reach significance $F(4,30)=$ $1.491, p=.23$. (see Figure 2.7).

After maintenance responding subjects completed a progressive ratio schedule with three doses of heroin: $0.05 \mathrm{mg} \mathrm{kg}^{-1}, 0.1 \mathrm{mg} \mathrm{kg}^{-1}$ and $0.2 \mathrm{mg} \mathrm{kg}^{-1}$. A repeated measures ANOVA failed to reveal a significant main effect of genotype $F(2,13)=1.345, p=.30$, or dose $F(2,26)=.56$, $p=.58$ on infusions. Additionally, the genotype $\mathrm{p}^{*}$ dose interaction failed to reach significance $F(4,26)=1.33, p=.29$ (see Figure 2.8). It should be noted that due the lengthy nature of drug self-administration experiments some subjects lost catheter patency before completing the entire experi- 
ment; therefore, the sample sizes decreased as the experiment progressed.

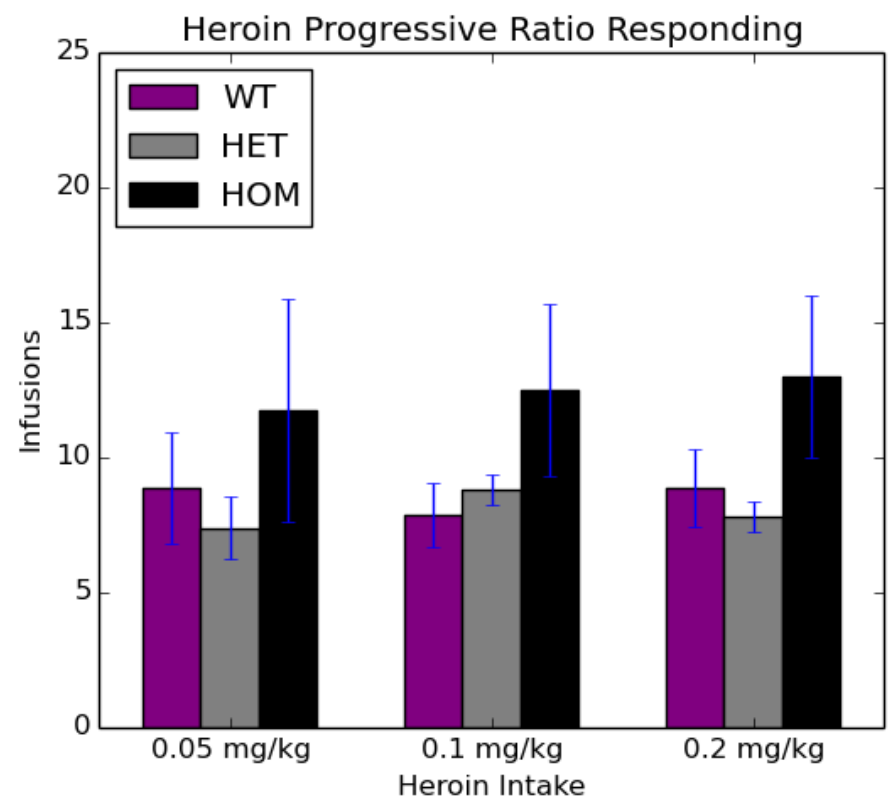

Figure 2.8: Average (+SEM) responding across varying MDMA doses on a progressive ratio schedule for $\operatorname{HOM}(n=4), \operatorname{HET}(n=5)$ and WT $(n=7)$ subjects.

As described above latency to begin feeding was measured to evaluate anxiety before animals began drug self-administration. Correlation analysis was conducted to compare latency to begin feeding with average heroin self-administration maintenance and progressive ratio responding. Neither analysis revealed a significant correlation between latency to begin feeding and heroin self-administration maintenance or progressive ratio responding, $r(15)=-.09, p=.74$, and $r(14)=.38, p=.15$, respectively (see Figures 2.9 and 2.10). 


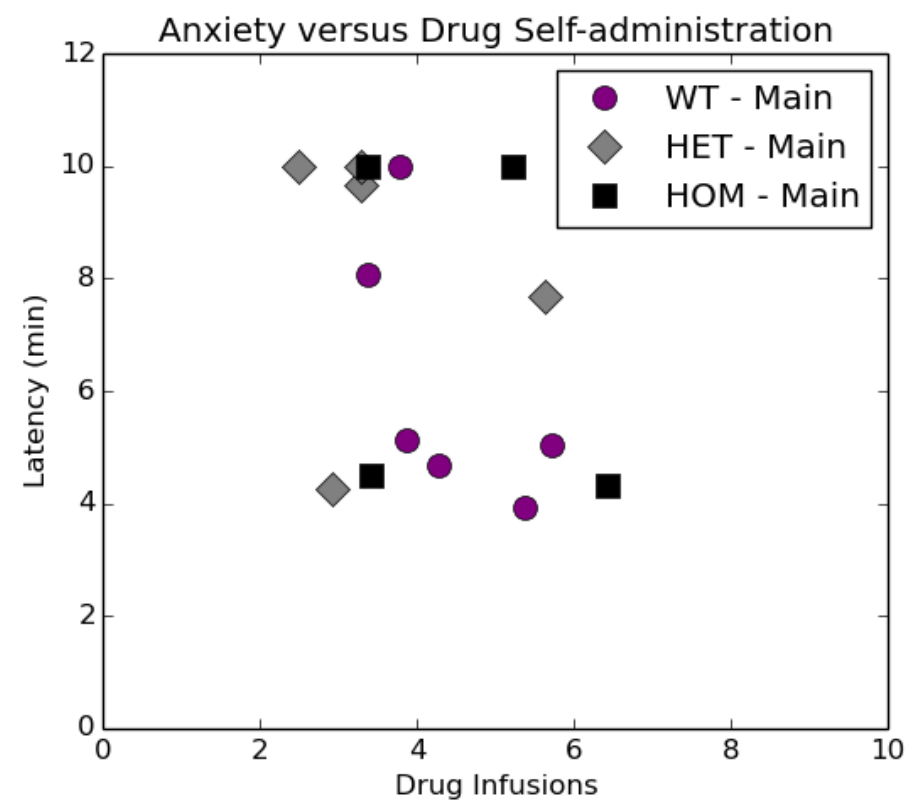

Figure 2.9: Latency to begin feeding (min) plotted against average maintenance responding (infusions).

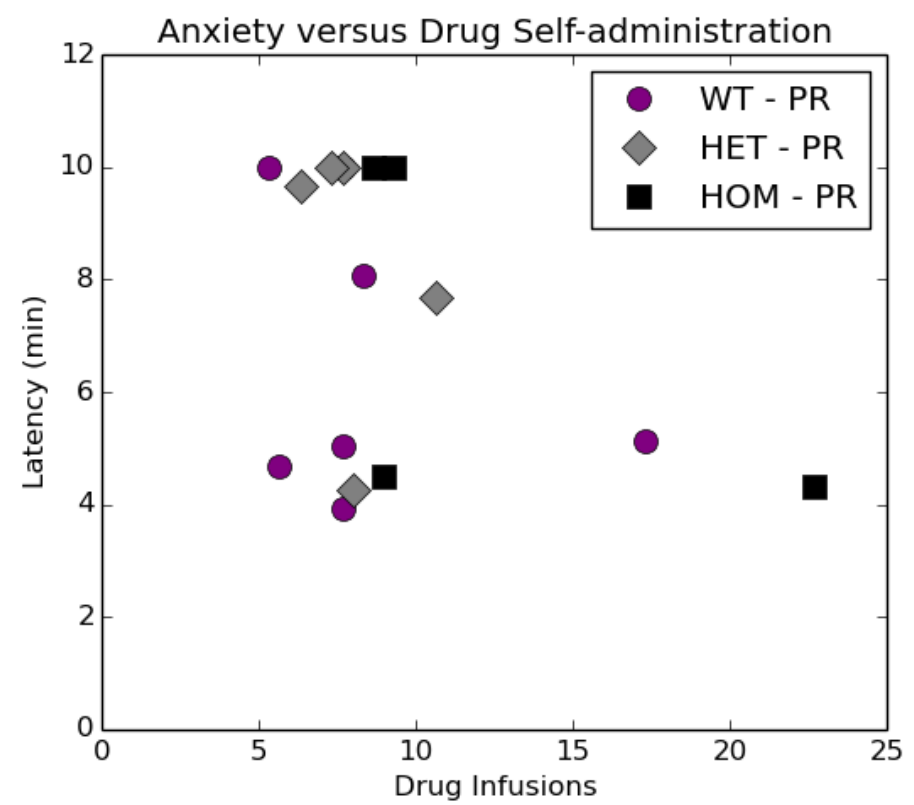

Figure 2.10: Latency to begin feeding ( $\mathrm{min}$ ) plotted against average progressive ratio responding (infusions). 


\subsection{Discussion}

Evaluation of latency in the novelty suppressed feeding paradigm provided a measure of anxiety; however, for the animals that underwent this testing before MDMA drug self-administration comparison of group averages did not reveal a difference between the genotypes. This result differs from previous research where significantly greater anxiety was correlated with reduction in the serotonin transporter (Olivier et al., 2008). Disagreement between these two studies may be due to slight differences in the experimental procedure. Prior to commencing the current novelty suppressed feeding experiment a pilot study was undertaken to provide familiarity with the experimental logistics before running the large cohort of animals that would go on to drug self-administration. In the pilot study, utilising a brightly lit open field to evaluate latency to begin feeding as specified in Olivier et al. (2008), none of the animals commenced feeding when presented with a food pellet within the duration of the test (10 minutes). Therefore, a slight procedural variation was included where the animals were moved into the experimental room 1 hour prior to beginning of the experiment. Additionally, the lights in the room were dimmed throughout the entirety of the experiment. This slight difference may provide an explanation as to the differing result between the two experiments.

For animals that underwent novelty suppressed feeding before heroin drug self-administration a nonsignificant trend emerged in latency to begin feeding amongst the genotypes. That is, the HOM and HET groups showed greater latency to begin feeding compared to the WT group. This does follow previous research (Olivier et al., 2008) however it should be noted that the post hoc comparisons failed to reach statistical significance. Considering very similar methods were used to run the novelty suppressed feeding experiments this differential result was not expected and may be due to natural variation between discrete groups of animals. Alternatively, minor changes in the experimental conditions may have increased 
the variability in the measured response; regardless the direction of the effect was the same in both novelty suppressed feeding experiments. This also suggests that levels of anxiety, at least as measured by novelty suppressed feeding, may not be a particularly robust read-out parameter.

To further investigate the relationship between levels of anxiety and heroin self-administration novelty suppressed feeding latency was compared with both maintenance and progressive ratio responding. Correlation analysis failed to revealed a significant relationship between levels of anxiety (increased latency) and either maintenance or progressive ratio responding for heroin.

Importantly, the groups of animals that completed MDMA drug selfadministration did not show differences in anxiety but demonstrated robust differences in MDMA drug self-administration. The animals that completed heroin drug self-administration showed a nonsignificant trend towards an increase in anxiety however there were no corresponding differences in heroin drug self-administration. If higher levels of anxiety, as demonstrated by HOM (and sometimes HET) subjects, were driving drug self-administration behaviour then these subjects would have shown facilitated drug self-administration for both MDMA and heroin. In fact when subjects self-administered heroin no differences emerged between the genotypes.

Regarding acquisition of MDMA self-administration a genotype effect emerged with HOM subjects acquiring MDMA drug self-administration significantly faster than both the HET and WT groups. Percentage of acquisition followed the predicted pattern with $100 \%$ of HOM subjects compared to $89 \%$ of HET subjects and only $50 \%$ of WT subjects. These results mirror findings from a pilot study conducted by our group where HOM and WT animals showed a similar pattern of responding when selfadministering MDMA (Oakly, Brox, Schenk, \& Ellenbroek, 2014). Additionally, with only half of WT subjects acquiring MDMA self-administration this result aligns with previous research where "normal" animals 
(those with intact serotonergic function) only acquire MDMA drug selfadministration about half of the time (Schenk et al., 2012). Importantly, the current result extends this pattern of responding, previously demonstrated in Sprague-Dawley rats, to a Wistar strain (SERT KO). While the comparison between the HET and WT groups did not technically reach significance the pattern of responding suggests that HET, like HOM, animals are more sensitive to the effects of MDMA as is reflected in their increased responding.

Beyond MDMA acquisition when animals were tested on FR1, FR2 and FR5 schedules of reinforcement the HOM group was more willing to increase their responding for additional drug infusions across FR schedules compared to both the HET and WT groups. When the genotypes were evaluated individually both the HOM and HET groups significantly increased their responding on a FR5 schedule; however, the WT group did not show a difference in responding across the FR schedules. After MDMA maintenance animals were effectively asked, "How hard are you willing to work for another drug infusion?" with a progressive ratio schedule of responding; however analysis only revealed a main effect of dose. Evaluation of each genotype across the drug doses revealed that both HOM and HET groups showed higher responding as dose increased while there were no differences for the WT group. These results suggest that a disrupted serotonergic function plays a role in increased responding for MDMA.

Taken together, the MDMA self-administration results suggest that a reduction in the SERT plays an important role in the acquisition, maintenance and progressive ratio responding for MDMA. Both HOM and HET groups consistently self-administered more MDMA compared with the WT group over a variety of FR schedules suggesting that these groups are more sensitive to the effects of MDMA and this sensitivity is driving their increased responding. Genotype differences for MDMA selfadministration are likely mediated by the complex interaction of serotonin 
and dopamine. That is, for animals with intact SERT function (WT) when MDMA is self-administered it acts on the SERT by blocking reuptake and facilitating reverse transport. This leads to increased levels of 5-HT in the synapse. In addition to action on the SERT MDMA also targets the dopamine transporter (DAT) (to a lesser degree). With previous research to support the idea that 5-HT release can have an inhibitory effect on dopamine neurotransmission this may explain why only about half of WT animals go on to acquire MDMA drug self-administration (Alex \& Pehek, 2007; Baumann et al., 2011; Di Matteo, De Blasi, Di Giulio, \& Esposito, 2001; Rothman \& Baumann, 2006). Conversely, for HOM subjects that completely lack the SERT when MDMA is self-administered there is no protein for MDMA to target and facilitate reverse transport of 5-HT back into the synapse. For this reason MDMA targets the DAT producing DA release into the synapse. As described earlier HOM animals, because they lack the SERT, have increased basal levels of 5-HT in specific brain regions that lead to 5-HT receptor downregulation. Therefore, in HOM animals MDMA may lead a tonic increase in 5-HT compared to the rapid phasic increase seen in WT animals. This result is consistent with a study where 5HT neurotransmission was disrupted via neurotoxic lesions (5,7 dihydroxytryptamine) in Sprague Dawley rats; MDMA self-administration was facilitated in these animals and coincided with a reduced 5-HT phasic response (Bradbury et al., 2014).

It should be noted that MDMA self-administration has been investigated in mice with reduced SERT function; WT animals self-administer MDMA reliably however SERT KO mice fail to demonstrate responding for MDMA above saline levels (Trigo et al., 2007). It has been suggested that this may be due to differences in the pharmacological targets of MDMA between the two species. As described earlier MDMA facilitates 5-HT release in rats and humans; however, in mice MDMA influences levels of 5-HT to a lesser degree. Instead, MDMA increases dopamine neurotransmission in mice and these elevated levels can lead to dopamine neu- 
rotoxicity (Easton \& Marsden, 2006). Unfortunately, this explanation is insufficient in describing why rats and mice with reduced SERT show differential patterns of MDMA self-administration. Interestingly, mice with reduced SERT demonstrate cocaine self-administration similar to WT animals (Thomsen, Hall, Uhl, \& Caine, 2009); if elevated dopamine release was inhibiting MDMA self-administration in SERT KO mice it would be expected to have the same effect on cocaine self-administration. Additional studies have investigated the influence of both serotonergic and dopaminergic disruption on cocaine reward and have indicated that there may be a complex relationship between the serotonin and dopamine neurotransmitter systems. That is, mice with reduced SERT or dopamine transporter (DAT) function both demonstrate cocaine induced conditioned place preference (CPP); these results suggest that the 5-HT and DA systems may play a compensatory role in the lifelong absence of the other transporter (Hagino et al., 2011; Sora et al., 1998). It is only with a double knockout that has neither SERT nor DAT function that cocaine CPP is effectively eliminated (Sora et al., 2001). It has been suggested that differences in MDMA self-administration between SERT rats and mice may be due to potentiated dopaminergic effects. That is, in rats these lead to enhanced MDMA self-administration but in mice they lead to dopamine neurotoxicity which inhibits self-administration behaviour. Interestingly, research has elegantly demonstrated that SERT mice (HOM and WT) show similar increases in dopamine in the nucleus accumbens when evaluated via microdialysis (Trigo et al., 2007). This result directly opposes the idea that MDMA leads to dopamine neurotoxicity in mice leading to diminished MDMA self-administration behaviour. Considering these findings as a whole it is possible that there are inherent differences in the serotonergic systems of rats and mice with a reduction in the SERT; studies indicate that specific serotonin receptor density varies between the two species (Fabre et al., 2000; Shanahan et al., 2009). Additionally, there may be fundamental differences in MDMA effects between rats and mice with 
reduced SERT; for example, HOM mice show spontaneous serotonin syndrome behaviours (Straub tail, tremors, tics) whereas HOM rats do not (Kalueff, LaPorte, \& Murphy, 2008). This finding is interesting considering both HOM rats and mice show elevated levels of extracellular 5-HT after MDMA treatment. At this time, even with current and historical data, we are unable to fully explain the differential effect between SERT rats and mice when they self-administer MDMA.

When HOM, HET and WT groups began heroin drug self-administration there were no significant differences between the genotypes over the first 15 testing sessions. However, when the data was evaluated for the first half of that period (Days 1-7) compared to the second half (Days 8-15) a significant main effect of Session emerged. Regardless of genotype, animals increased their responding over sessions. On maintenance schedules, FR1, FR2 and FR5, there were no significant differences in responding between the genotypes. Additionally, there was no difference between the genotypes when responding on a progressive ratio schedule across several drug doses. It was hypothesised that reduced SERT function leads to a sensitised dopaminergic response to drugs of abuse. However, if this were true then HOM, and most likely HET, subjects would have demonstrated greater heroin self-administration compared to the WT. Instead, no genotype differences emerged throughout the whole of heroin self-administration including varying doses and schedules of responding. Therefore, the theory that animals with reduced SERT function have a sensitised dopaminergic system may not be true. However, it is important to note that, compared to MDMA, heroin is a powerful drug and it is possible that potential genotype differences may be difficult to detect with such a potent reinforcer. Cocaine self-administration in rats with reduced SERT function is facilitated similarly to the MDMA self-administration results presented in this chapter. Considering cocaine and MDMA target both the SERT and DAT it is possible that this direct stimulation of the dopaminergic system then drives their respective self-administration. Alternatively, 
the heroin mechanism of action is inherently different as it acts indirectly to stimulate dopamine release.

Regarding the drug self-administration experiments as a whole it is readily apparent that reduction in the SERT influences drug self-administration behaviour very differently depending on the type of drug that is self-administered. MDMA drug self-administration was facilitated in animals with reduced SERT function whereas heroin drug self-administration did not differ between the genotypes. This result is in line with previous research that demonstrated facilitated cocaine self-administration in subjects with reduced SERT function; specifically, the desensitisation of a specific serotonergic autoreceptor $\left(5-\mathrm{HT}_{1 \mathrm{~A}}\right)$ leading to facilitated cocaine selfadministration (Homberg et al., 2008). Another study in rats with reduced SERT function demonstrated that cocaine facilitated a significantly smaller increase in extracellular 5-HT in the nucleus accumbens and hippocampus compared to WT animals. This study also demonstrated that cocaine treatment did not lead to differing levels of dopamine and norepinephrine between HOM and WT animals (Verheij, Karel, Cools, \& Homberg, 2014). Together with the current results this research points to the existence of a complex relationship between the serotonin, dopamine and norepinephrine neurotransmitter systems which in turn drives drug self-administration in animals with reduced SERT.

These studies provide insight into how reduced SERT function may influence the development of drug addiction. That is, because reduction in the SERT has been linked to other psychiatric disorders it was initially posited that a reduction in the SERT would constitute a risk factor in the development of drug addiction to all drugs of abuse. However, the results of this suite of experiments provide evidence counter to this argument. Namely, that a reduction in the SERT facilitates the self-administration of MDMA but not for heroin. As described in the General Introduction there is growing evidence to implicate a reduction in the SERT in the development of drug addiction. The current MDMA self-administration data pro- 
vides support for this theory. Conversely, the heroin self-administration data suggests that a reduction in the SERT does not contribute to the development of opioid addiction. Interestingly, a portion of the human clinical research into heroin addiction supports the idea that a reduction in the SERT confers susceptibility to developing addictive behaviours (Gerra et al., 2004; Saiz et al., 2008; Tan, Yeo, Ho, Tay, \& Tan, 1999; Yang, Kavi, Wang, $\mathrm{Wu}$, \& Hao, 2012); other research shows no such relationship (Kotler et al., 1999; T. Li et al., 2002; Saiz et al., 2009). A review of these studies suggests that these mixed results may be due to small sample sizes, variations in the SERT polymorphism between different ethnic groups and the fact that there are relatively few studies investigating the specific relationship between reduced SERT function and heroin addiction (Cao et al., 2013). Therefore, the current preclinical data fit with a portion of the human data where a reduction in the SERT does not appear to be a risk factor when it comes to heroin addiction. Only additional research in larger and more diverse groups of humans will elucidate this relationship.

Overall, this series of experiments provided an opportunity for evaluating the effect of disrupted serotonergic neurotransmission on anxiety and drug self-administration. Interestingly, animals with reduced SERT function showed facilitated MDMA, but not heroin, drug self-administration compared to those with intact SERT function. While these results are interesting they beg the question: "Why do animals with reduced SERT function show increased MDMA self-administration?". The incentive-sensitisation theory of drug addiction suggests that increased drug taking results from a sensitised "reward" network where the behaviour is increasingly reinforced; that is drug taking escalates due to the "positive" reinforcing properties the drug (Robinson \& Berridge, 1993, 2001). Conversely, others have posited that increased drug taking is related to the "negative" effects of the withdrawal syndrome that increase over time. This theory is supported by the fact that individuals continue to take increasing amounts of the drug and this may postpone experience of the withdrawal 
syndrome (Koob, 1992; Koob et al., 2009, 1998). In an effort to determine which theory of drug addiction is applicable groups of HOM, HET and WT animals completed a series of locomotor activity and conditioned taste aversion experiments. These two paradigms have been widely used to assess aspects of drug addiction including reinforcing properties and negative effects. They are described fully in Chapter 3. 
CHAPTER 2. NOVELTY SUPPRESSED FEEDING AND DRUG SELF-ADMINISTRATION 


\section{Chapter 3}

\section{Locomotor Activity and Conditioned Taste Aversion}

\subsection{Introduction}

The drug self-administration experiments described in Chapter 2 demonstrated a strong genotype effect with MDMA, but not heroin, begging the question,"What is driving the effect?". Are the HOM and HET animals self-administering significantly more MDMA compared to the WT because of the positive, reinforcing effects of the drug? Or do they take more to stave off the negative consequences that can accompany the withdrawal syndrome? To investigate these questions a series of experiments was conducted. Specifically, groups of HOM, HET and WT animals were treated with MDMA or heroin and their locomotor activity or conditioned taste aversion was measured.

Assessing locomotion in experimental animals has become a hallmark in preclinical research especially when evaluating drug effects (Walsh \& Cummins, 1976). It allows a rapid and precise measure of how the drug influences motor movement and provides a behavioural correlate to drug induced neurochemical changes in the brain (Beninger, 1983; Pijnenburg \& van Rossum, 1973). It is widely posited that drug induced locomotor 
activity is driven by activation of the mesolimbic dopamine pathway. This is evidenced by several studies that have demonstrated increased locomotion after treatment with DA stimulating substances like D-amphetmaine, cocaine and apomorphine (Fray, Sahakian, Robbins, Koob, \& Iversen, 1980; Isaacson, Yongue, \& Mcclearn, 1978; Lyon \& Robbins, 1975). Additionally, other studies have shown that DA antagonists or lesions created with 6hydroxydopamine (6-OHDA) reliably decrease psychostimulant induced locomotor activity in laboratory animals (Anden, Butcher, Corrodi, Fuxe, \& Ungerstedt, 1970; Carlsson, Lindqvist, Magnusson, \& Waldeck, 1958; Ungerstedt, 1979). This is also true for MDMA; several studies have demonstrated that dopamine receptor antagonists decrease locomotor activity (Ball, Budreau, \& Rebec, 2003; Daniela, Brennan, Gittings, Hely, \& Schenk, 2004). Additionally, treatment with opioids like heroin and morphine activate the mesolimbic dopamine pathway and facilitate dose dependent locomotor activity (Kalivas \& Duffy, 1987; Ranaldi, Egan, Kest, Fein, \& Delamater, 2009; Vanderschuren \& Kalivas, 2000). As described in the General Introduction dopaminergic activity in this same pathway is thought to underlie the initially rewarding properties of drugs of abuse (Everitt et al., 2008; Koob et al., 1998; Koob \& Volkow, 2010; Nestler, 1996, 2001; Robbins \& Everitt, 1996). Therefore, in order to determine if animals with reduced SERT are more sensitive to the reinforcing properties of drugs of abuse locomotor activity was measured after MDMA or heroin treatment.

Conversely, to assess sensitivity to the negative effects of drugs of abuse groups of animals were tested in a conditioned taste aversion paradigm. Conditioned taste aversion develops when a drug of abuse is delivered systemically after an animal has had access to a novel, palatable sucrose solution. Under normal circumstances animals will readily drink sucrose when given the opportunity. However, when paired with a drug of abuse animals will reliably avoid the sucrose solution when given a choice between it and water (Cappell \& LeBlanc, 1977; Goudie, 1979). Previous research suggests that this phenomena demonstrates that drugs of abuse 
may possess both positive and negative effects. This is evidenced by the fact that drugs that are readily self-administered by animals also cause conditioned taste aversion when paired with a desirable solution like sucrose (Hunt \& Amit, 1987). Therefore, to evaluate the influence of negative drug effects development of conditioned taste aversion was evaluated after MDMA or heroin treatment.

\subsubsection{Predictions}

Prediction 1: As subjects with reduced SERT appear to have heightened sensitivity to MDMA drug effects (i.e. facilitated MDMA selfadministration described in Chapter 2) it is predicted that MDMA induced locomotor activity will be inversely related to SERT function $(\mathrm{HOM}>\mathrm{HET}>\mathrm{WT})$.

Prediction 2: As subjects with reduced SERT appear to have heightened sensitivity to MDMA drug effects (i.e. facilitated MDMA selfadministration described in Chapter 2) it is predicted that MDMA induced conditioned taste aversion will be inversely related to SERT function $(\mathrm{HOM}>\mathrm{HET}>\mathrm{WT})$.

Prediction 3: As heroin self-administration behaviour was similar across genotypes (Chapter 2) it is predicted that heroin induced locomotor activity will not differ between HOM, HET and WT groups.

Prediction 4: As heroin self-administration behaviour was similar across genotypes (Chapter 2) it is predicted that heroin induced conditioned taste aversion will not differ between HOM, HET and WT groups. 


\subsection{Methods}

\subsubsection{Locomotor Activity}

\section{Subjects}

All experimental procedures were approved by the Animal Ethics Committee at Victoria University of Wellington. Subjects were male Wistar rats, homozygous (SERT $\left.{ }^{-/-}, \mathrm{HOM}\right)$, heterozygous (SERT ${ }^{+/-}, \mathrm{HET}$ ) and wild type (SERT ${ }^{+/}, \mathrm{WT}$ ), weighing 300-400 $\mathrm{g}$ at the time of the experiments described below. Breeding occurred in the vivarium at Victoria University of Wellington and animals were housed in groups of 3-4 until five days prior to the commencement of testing. During testing animals were housed in pairs (same genotype) in a temperature $\left(19-21{ }^{\circ} \mathrm{C}\right)$ and humidity $(55 \%)$ controlled room that was maintained on a 12 hour light-dark cycle with lights on at 0700 . Testing occurred during the light phase beginning each day at 0800 . Food and water was available ad libitum except during testing.

\section{Apparatus}

Locomotor activity testing was conducted as previously described (Bradbury, Gittings, \& Schenk, 2012). Data were collected using sixteen clear Plexiglas open field chambers, $42 \times 42 \times 30 \mathrm{~cm}$, (Med Associates Inc., USA; model ENV-515) housed in sound attenuating boxes. Each chamber was equipped with four sets of sixteen infrared sensors, at two different heights, that created a lattice of beams dividing the space into squares measuring $25 \times 25 \mathrm{~mm}$. Ambulatory counts were recorded where the interruption of three consecutive, adjacent beams defined one "ambulatory count". During testing a white noise generator was utilised to mask any noise outside the boxes. 


\section{Testing Schedule}

Animals were transferred to the testing room in their home cages. Weight was recorded for each animal. With the white noise generator on they were placed into the locomotor activity chambers. After thirty minutes subjects were injected with either drug or saline and placed back into the chambers for $60 \mathrm{~min}$. After the experiment animals were placed back into their home cages and returned to the housing room.

Groups of 8 animals per genotype were counterbalanced across chambers where each animal used the same chamber for all sessions. Using a within subjects design (1 dose per week) locomotor activity was measured for four counterbalanced doses of MDMA: $0.0 \mathrm{mg} \mathrm{kg}^{-1}$ (saline), $2.5 \mathrm{mg} \mathrm{kg}^{-1}$, $5.0 \mathrm{mg} \mathrm{kg}^{-1}$ and $10.0 \mathrm{mg} \mathrm{kg}^{-1}$. Identical testing procedures were used for three counterbalanced doses of heroin: $0.0 \mathrm{mg} \mathrm{kg}^{-1}$ (saline), $0.5 \mathrm{mg} \mathrm{kg}^{-1}$ and $1.0 \mathrm{mg} \mathrm{kg}^{-1}$.

\section{Drugs}

Drug solutions were prepared by mixing 3,4-methylenedioxymethamphetamine or heroin $\mathrm{HCl}$ (BDG Synthesis, Wellington, New Zealand), in powder form, with saline. Both MDMA and heroin were administered subcutaneously (s.c.) in a volume of $1.0 \mathrm{mg} \mathrm{kg}^{-1}$.

\subsubsection{Conditioned Taste Aversion}

\section{Subjects}

All experimental procedures were approved by the Animal Ethics Committee at Victoria University of Wellington. Subjects were male Wistar rats, homozygous (SERT $\left.{ }^{-/-}, \mathrm{HOM}\right)$, heterozygous $\left(\mathrm{SERT}^{+/-}, \mathrm{HET}\right)$ and wild type $\left(\mathrm{SERT}^{+/+}, \mathrm{WT}\right)$, weighing $300-450 \mathrm{~g}$ at the time of the experiments. Breeding occurred in the vivarium at Victoria University of Wellington and animals were housed in groups until they underwent the experimen- 
tal paradigm described below. Animals were transferred to individual cages housed in a temperature $\left(19-21^{\circ} \mathrm{C}\right)$ and humidity (55\%) controlled room that was maintained on a 12 hour light-dark phase with lights on at 0700 . Testing occurred during the light phase beginning each day at 0900. Food was available ad libitum but water availability was restricted according to the procedure described below.

\section{Apparatus}

Two days before the experiment began subjects were transferred to individual home cages where they remained for the duration of experimental testing. They were then water deprived for 24 hours before beginning the six day testing period.

\section{Testing Schedule}

The experimental methods follow a procedure described previously (Fenu, Cadoni, \& Di Chiara, 2010). The testing period consisted of three phases: training, conditioning and testing. First, subjects underwent training for three days (Days 1, 2 and 3). Each day animals were given access to a water bottle for $30 \mathrm{~min}$. Water bottles were weighed before and after drinking to calculate intake and ensure that animals were drinking water when it was available. Second, subjects completed the conditioning phase for two days (Days 4 and 5). Before testing subjects were weighed and then given access to a bottle containing a 5\% sucrose solution for $30 \mathrm{~min}$. After $30 \mathrm{~min}$ had elapsed subjects were injected with MDMA or saline. Finally, testing was conducted on Day 6. Subjects were given access to both a bottle filled with water and one filled with $5 \%$ sucrose solution. All water and sucrose bottles were counterbalanced across the left and right sides of the cage throughout the experiment. Conditioned taste aversion was calculated as the intake of sucrose divided by total fluid intake (sucrose + water).

Groups of 8 animals per genotype were randomly assigned to a treat- 
ment condition; conditioned taste aversion was measured for three doses of MDMA: $0.0 \mathrm{mg} \mathrm{kg}^{-1}$ (saline), $2.5 \mathrm{mg} \mathrm{kg}^{-1}$ and $5.0 \mathrm{mg} \mathrm{kg}^{-1}$. Identical testing procedures were used for used for three counterbalanced doses of heroin: $0.0 \mathrm{mg} \mathrm{kg}^{-1}$ (saline), $0.5 \mathrm{mg} \mathrm{kg}^{-1}$ and $1.0 \mathrm{mg} \mathrm{kg}^{-1}$.

\section{Drugs}

Drug solutions were prepared by mixing 3,4-methylenedioxymethamphetamine or heroin $\mathrm{HCl}$ (BDG Synthesis, Wellington, New Zealand), in powder form, with saline. Both MDMA and heroin were administered via intraperitoneal (i.p.) injection in a volume of $1 \mathrm{mg} \mathrm{kg}^{-1}$.

\subsection{Results}

\subsubsection{Locomotor Activity}

\section{MDMA}

Levels of basal locomotor activity were inversely related to SERT function $(\mathrm{HOM}>\mathrm{HET}>\mathrm{WT})$ reflecting increased exploratory behaviour in HOM subjects (data not shown). Locomotor activity following a drug injection was recorded over $60 \mathrm{~min}$ and is reported as total ambulatory counts. A repeated measures ANOVA was conducted with dose (saline, MDMA 2.5, MDMA 5.0 and MDMA 10.0) as a within subjects variable and genotype (HOM, HET, WT) as a between subjects variable. This analysis revealed a significant main effect of dose $F(3,63)=13.81, p<.001$, and a significant main effect of genotype $F(2,21)=3.69, p=.04$. The dose* genotype was also significant $F(6,63)=4.48, p=.001$ (see Figure 3.1).

Subsequently, one-way ANOVA post hoc analysis of locomotor activity was conducted for each drug dose. Saline treatment elicited a significant difference between the genotypes $F(2,21)=3.94, p=.04$, with HOM subjects registering more ambulatory counts compared to WT. Analysis of 


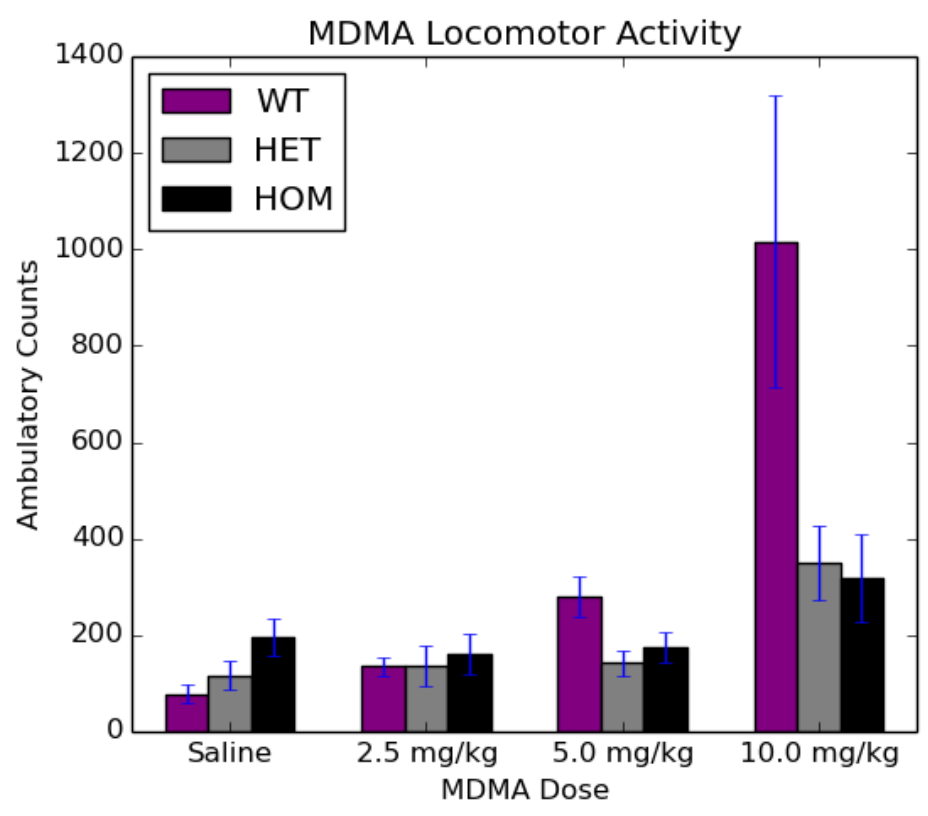

Figure 3.1: Average $(+\mathrm{SEM})$ locomotor activity (ambulatory counts) for $\operatorname{HOM}(\mathrm{n}=8)$, HET $(\mathrm{n}=8)$ and WT $(\mathrm{n}=8)$ subjects.

MDMA $2.5 \mathrm{mg} \mathrm{kg}^{-1}$ elicited locomotor activity revealed no differences between the genotypes, $F(2,21)=.16, p=.86$; however, when animals were treated with MDMA $5.0 \mathrm{mg} \mathrm{kg}^{-1}$ there was a significant difference in ambulatory counts between the genotypes $F(2,21)=4.46, p=.02$, with WT animals demonstrating higher ambulatory counts compared to HET. Lastly, analysis of MDMA $10.0 \mathrm{mg} \mathrm{kg}^{-1}$ elicited locomotor activity revealed a significant difference between the genotypes $F(2,21)=4.4, p=.03$; WT animals showed significantly greater locomotor activity when compared to both HOM and HET groups (see Figure 3.1 and Table 3.1).

Upon further investigation within each genotype HOM subjects failed to show a significant difference between the doses $F(3,28)=1.64, p=.20$. However, HET subjects showed a significant difference between doses $F(3$, $28)=5.07, p=.006$, with significantly greater locomotor activity when treated with MDMA $10.0 \mathrm{mg} \mathrm{kg}^{-1}$ compared to the other doses. Similarly, 


\begin{tabular}{|c|c|c|c|c|}
\hline & Saline & MDMA 2.5 & MDMA 5.0 & MDMA 10.0 \\
\hline HOM - HET & 0.18 & 0.89 & 0.78 & 0.99 \\
\hline HOM - WT & $\mathbf{0 . 0 3}^{*}$ & 0.87 & 0.10 & $\mathbf{0 . 0 4}^{*}$ \\
\hline HET - WT & 0.65 & 1.00 & $\mathbf{0 . 0 2}$ & 0.05 \\
\hline
\end{tabular}

Table 3.1: MDMA locomotor activity: post hoc comparisons. ${ }^{*}$ Denotes statistical significance.

WT subjects showed a significant difference between the doses $F(3,28)=$ $8.02, p=.001$; like the HET group WT animals showed significantly greater locomotor activity when treated with MDMA $10.0 \mathrm{mg} \mathrm{kg}^{-1}$ compared to the other doses (see Table 3.2).

\begin{tabular}{|c|c|c|c|}
\hline & HOM & HET & WT \\
\hline Saline - MDMA 2.5 & 0.97 & 0.99 & 0.99 \\
\hline Saline - MDMA 5.0 & 0.99 & 0.98 & 0.79 \\
\hline Saline - MDMA 10.0 & 0.42 & $\mathbf{0 . 0 1}^{*}$ & $\mathbf{0 . 0 0 1}^{*}$ \\
\hline MDMA 2.5 - MDMA 5.0 & 1.00 & 1.00 & 0.91 \\
\hline MDMA 2.5 - MDMA 10.0 & 0.22 & $\mathbf{0 . 0 2}^{*}$ & $\mathbf{0 . 0 0 2}^{*}$ \\
\hline MDMA 5.0 - MDMA 10.0 & 0.29 & $\mathbf{0 . 0 3}^{*}$ & $\mathbf{0 . 0 1}^{*}$ \\
\hline
\end{tabular}

Table 3.2: MDMA locomotor activity: Post hoc comparisons. *Denotes statistical significance.

\section{Heroin}

Locomotor activity following a drug injection was recorded over $60 \mathrm{~min}$ and is reported as total ambulatory counts. A repeated measures ANOVA was conducted with dose (saline, heroin 0.5 , and heroin 1.0) as a within subjects variable and genotype (HOM, HET, WT) as a between subjects variable. This analysis revealed a significant main effect of dose $F(2,42)$ $=6.53, p=.003$; however the main effect of genotype, $F(2,21)=0.58, p=$ 
.60 , and the dose ${ }^{*}$ genotype interaction failed to reach significance $F(4,42)$ $=1.45, p=.24$ (see Figure 3.2).

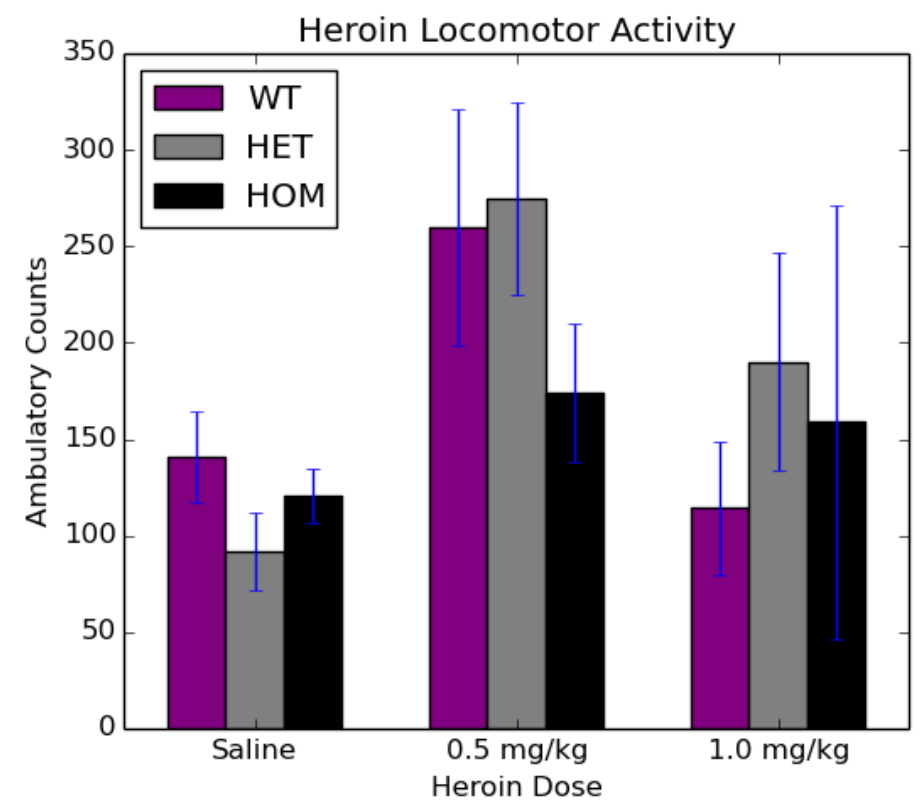

Figure 3.2: Average (+SEM) locomotor activity (ambulatory counts) for $\operatorname{HOM}(\mathrm{n}=8)$, HET $(\mathrm{n}=8)$ and WT $(\mathrm{n}=8)$ subjects.

\subsubsection{Conditioned Taste Aversion}

\section{MDMA}

Conditioned taste aversion to a sucrose solution was calculated by dividing sucrose consumption by total consumption (sucrose + water) on the final day of testing. A two-way ANOVA was conducted with dose (saline, MDMA 2.5, MDMA 5.0) and genotype (HOM, HET, WT) as betweensubjects variables. This analysis revealed a significant main effect of dose $F(2,62)=61.7, p<.001$. The main effect of genotype failed to reach significance $F(2,62)=.89, p=.42$; interestingly the dose* genotype interaction 
did not reach significance but may indicate a trend toward an effect $F(4$, $62)=2.17, p=.08$ (see Figure 3.3)

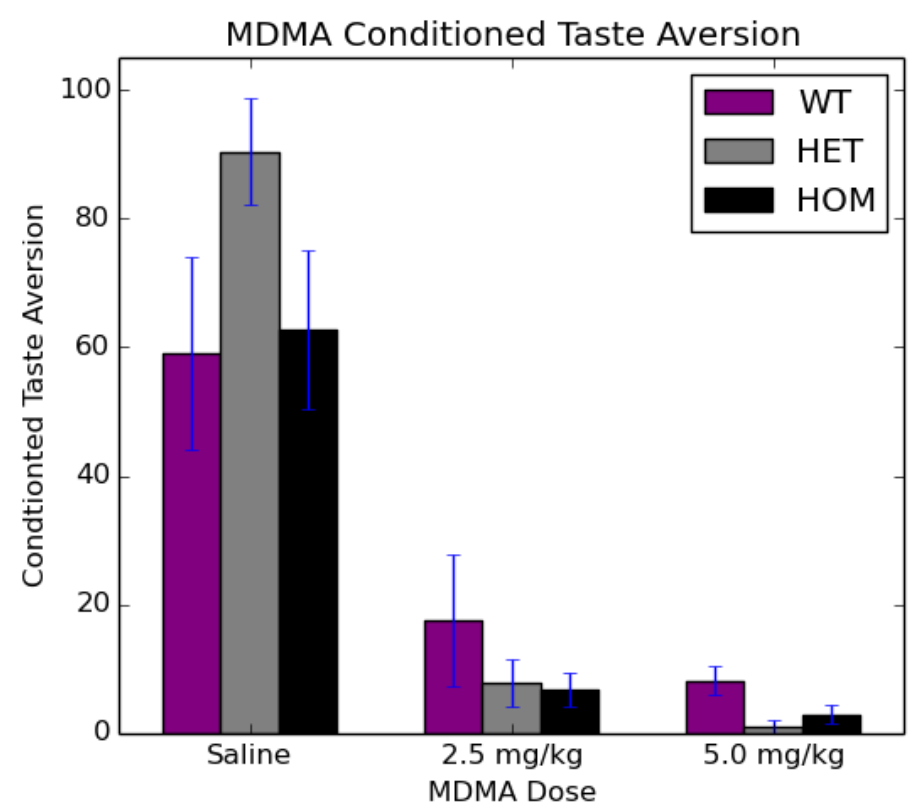

Figure 3.3: Average $(+S E M)$ conditioned taste aversion for HOM $(n=23)$, HET $(n=24)$ and WT $(n=24)$ subjects

Subsequently, one-way ANOVA post hoc analysis of conditioned taste aversion was conducted for each drug dose. Saline treatment failed to elicit a difference in conditioned taste aversion between the genotypes $F(2$, $20)=1.98, p=.16$, nor was the effect seen for animals treated with MDMA $2.5 \mathrm{mg} \mathrm{kg}^{-1} F(2,20)=.86, p=.44$. However, analysis revealed a significant difference between the genotypes treated with MDMA $5.0 \mathrm{mg} \mathrm{kg}^{-1} F(2$, $20)=5.09, p=.02$; HET animals showed significantly greater conditioned taste aversion when compared with WT. The HOM/HET and HOM/WT comparisons both failed to reach significance (see Figure 3.3 and Table 3.3).

Upon further investigation within each genotype HOM subjects showed a significant difference between the doses $F(2,20)=19.41, p<.001$. Rats showed significantly greater conditioned taste aversion when treated with 
MDMA $2.5 \mathrm{mg} \mathrm{kg}^{-1}$ and MDMA $5.0 \mathrm{mg} \mathrm{kg}^{-1}$ compared to saline. However, the MDMA $2.5 \mathrm{mg} \mathrm{kg}^{-1}$ and MDMA $5.0 \mathrm{mg} \mathrm{kg}^{-1}$ comparison failed to reach significance. The same pattern was seen for the HET and the WT animals (see Table 3.4).

\begin{tabular}{|c|c|c|c|}
\hline & Saline & MDMA 2.5 & MDMA 5.0 \\
\hline HOM - HET & 0.27 & 0.99 & 0.69 \\
\hline HOM - WT & 0.97 & 0.47 & 0.10 \\
\hline HET - WT & 0.19 & 0.54 & $\mathbf{0 . 0 2}^{*}$ \\
\hline
\end{tabular}

Table 3.3: MDMA Conditioned Taste Aversion: post hoc comparisons. ${ }^{*}$ Denotes statistical significance.

\begin{tabular}{|c|c|c|c|}
\hline & HOM & HET & WT \\
\hline Saline - MDMA 2.5 & $\mathbf{0 . 0 0}^{*}$ & $\mathbf{0 . 0 0}^{*}$ & $\mathbf{0 . 0 3}^{*}$ \\
\hline Saline - MDMA 5.0 & $\mathbf{0 . 0 0}^{*}$ & $\mathbf{0 . 0 0}^{*}$ & $\mathbf{0 . 0 1}^{*}$ \\
\hline MDMA 2.5 - MDMA 5.0 & 0.94 & 0.63 & 0.80 \\
\hline
\end{tabular}

Table 3.4: MDMA Conditioned Taste Aversion: Post hoc drug dose comparisons. ${ }^{*}$ Denotes statistical significance.

\section{Heroin}

Conditioned taste aversion to a sucrose solution was calculated by dividing sucrose consumption by total consumption (sucrose + water) on the final day of testing. A two-way ANOVA was conducted with dose (saline, heroin 0.5 , heroin 1.0) and genotype (HOM, HET, WT) as between-subjects variables. Both dose and genotype main effects failed to reach significance, $F(2,61)=1.77, p=.18$, and $F(2,61)=.38, p=.69$, respectively. Similarly, the dose ${ }^{*}$ genotype interaction failed to reach significance $F(4,61)=1.28, p$ $=.29$ (see Figure 3.4). 


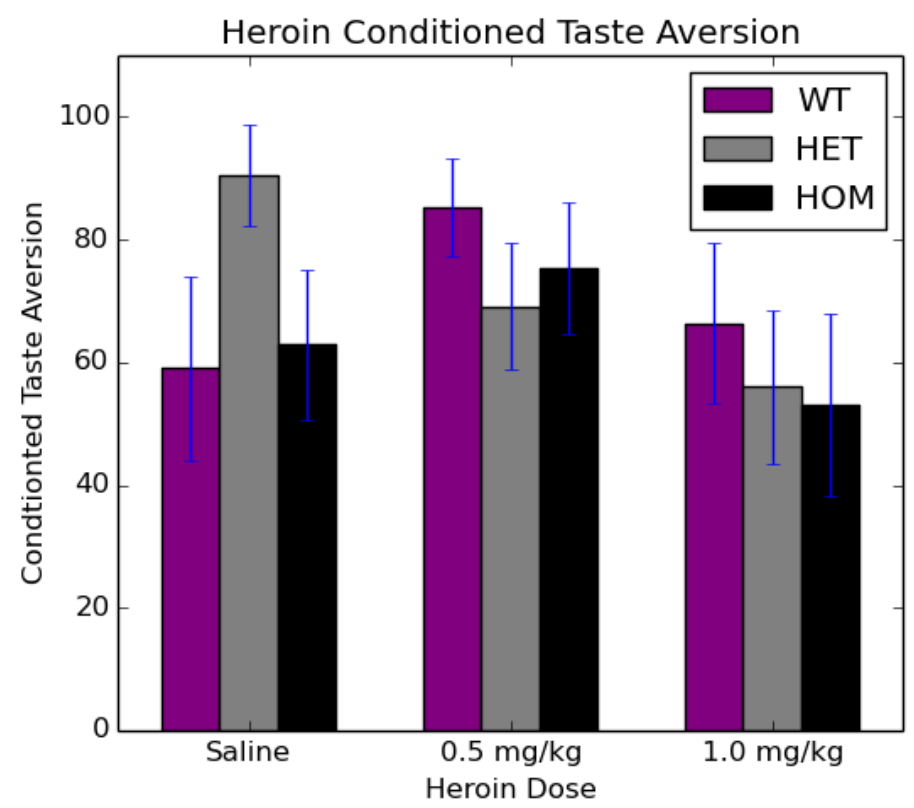

Figure 3.4: Average $(+S E M)$ conditioned taste aversion for HOM $(n=22)$, HET $(\mathrm{n}=24)$ and WT $(\mathrm{n}=24)$ subjects.

\subsection{Discussion}

Taken together the locomotor activity results demonstrate that MDMA differentially influences locomotion amongst the genotypes whereas heroin does not. Intriguingly and contrary to our predictions, we found that MDMA induced locomotor activity was highest in the WT and completely absent in the HOM rats (see Table 3.2). If heightened locomotion is an indicator of the rewarding properties of drugs of abuse this pattern of results would suggest that the WT subjects compared to the other groups are the most sensitive to MDMA. However, this conclusion is not in line with what was observed when HOM, HET and WT subjects self-administered MDMA as described in Chapter 2. Considering reduced SERT function coincided with facilitated MDMA self-administration it appeared that HOM (and HET) subjects were more sensitive to MDMA com- 
pared to the WT. These seemingly contradictory results can be explained by examining the neurochemical mechanism underlying MDMA induced locomotor activity. As mentioned above research has demonstrated that dopamine neurotransmission in the mesolimbic pathway underlies both the initial rewarding properties of drugs of abuse and locomotor activity. However, as our data seem to indicate, MDMA induced locomotor activity appears to be fundamentally different from other psychostimulants like amphetamine and cocaine; Homberg et al. (2008) demonstrated that HOM animals showed elevated locomotor activity after cocaine treatment (Homberg et al., 2008). Importantly, MDMA induced locomotor activity is partially driven by 5 -HT release and this may explain why WT subjects show heightened locomotor activity compared to subjects with reduced SERT function. This result is consistent with several studies that have implicated serotonin in the behavioural effects associated with MDMA, demonstrating that MDMA induced locomotor activity is driven by the release of 5-HT (Callaway \& Geyer, 1992; Callaway, Johnson, Gold, Nichols, \& Geyer, 1991; Callaway, Rempel, Peng, \& Geyer, 1992; Callaway, Wing, \& Geyer, 1990). More recent research has demonstrated that when SERT KO and WT rats were treated with MDMA both groups demonstrated higher levels of locomotor activity compared saline treated controls; interestingly, SERT KO animals treated with MDMA displayed delayed, lower levels of locomotor activity compared to those with intact SERT function (Lizarraga et al., 2014).

If MDMA induced locomotor activity is driven by 5-HT release then evaluating this behaviour may not provide an accurate measure of the dopaminergic activity and by extension the rewarding properties of these particular drugs of abuse. However, evaluation of baseline levels of locomotor activity may elucidate an important aspect of dopaminergic neurotransmission. That is, baseline levels of locomotor activity reveal significantly greater locomotion for HOM animals compared to WT; this pattern reflects differences in novelty induced locomotion which is related to 
dopamine release (Bromberg-Martin, Matsumoto, \& Hikosaka, 2010; Dulawa, Grandy, Low, Paulus, \& Geyer, 1999). Baseline comparisons then suggest that HOM animals, compared to WT, may have increased dopamine neurotransmission and this translates to greater levels of locomotor activity. This is consistent with heightened locomotor activity seen in HOM animals that have been treated with cocaine suggesting increased dopaminergic sensitivity (Homberg et al., 2008). Taken as a whole these results suggest that despite increased basal levels of locomotor activity in HOM animals it is primarily the release of 5-HT that drives MDMA induced locomotor activity.

Regarding heroin induced locomotor activity it was hypothesised that, considering there were no differences in heroin self-administration, a reduction in the SERT may not lead to a differential locomotor response between the genotypes. As predicted, locomotion was largely consistent across the doses for HOM, HET and WT groups. That is, animals, regardless of genotype showed low levels of locomotion when treated with saline. However, when treated with heroin $0.5 \mathrm{mg} \mathrm{kg}^{-1}$ animals showed a modest increase in locomotor activity; then locomotor activity decreased when the dose was increased to heroin $1.0 \mathrm{mg} \mathrm{kg}^{-1}$. Upon inspection of the raw data it became apparent that some subjects, when treated with heroin $1.0 \mathrm{mg} \mathrm{kg}^{-1}$ (the highest dose), showed no locomotor activity in the final minutes of the session (consecutive ambulatory counts of 0 ). This suggests a higher dose of heroin may have induced sedation to the point where little, if any, locomotor activity would have been produced. Therefore, it is unlikely that the lack of genotype effect is the result of an incorrect dose range but reflects a true null effect between the groups.

The MDMA conditioned taste aversion data are interesting and promising; they suggest that a genotype difference may exist depending on drug dose with reduced SERT function influencing a heightened conditioned taste aversion. It was predicted that reduction in the SERT would be inversely related to conditioned taste aversion with HOM subjects showing 
the largest effect. As described above comparisons failed to reach significance however this trend followed the initial prediction. Both MDMA doses dramatically decreased sucrose consumption across the genotypes with the higher dose producing the most dramatic shift in drinking behaviour. Considering this trend it would be useful to investigate how a lower dose (MDMA $1.0 \mathrm{mg} \mathrm{kg}^{-1}$ ) may influence the development of MDMA induced conditioned taste aversion.

Finally, when the development of heroin conditioned taste aversion was evaluated analysis revealed no differences between the groups. The HOM, HET and WT groups failed to demonstrate conditioned taste aversion for either drug dose. That is, the animals continued to prefer and consume the sucrose solution even after it had been paired with heroin. Initially, this was surprising considering research has demonstrated that almost all drugs of abuse can initiate the development of conditioned taste aversion. It is possible that this null effect was due to an insufficient association between the sucrose solution and the effect of the drug. Heroin induced central nervous system inhibition may have disrupted the process where the drug effect becomes associated with the sucrose solution. This may explain why when presented with a choice between sucrose and water the animals, regardless of genotype, continued to drink the sucrose. Importantly, prior research has demonstrated that conditioned taste aversion to sucrose failed to develop when animals were given morphine (Parker, 1995). This effect was then demonstrated again in a study utilising morphine to investigate the combination of deprivation state, drug type and caloric value of the palatable solution. They found that in water deprived animals morphine failed to elicit a conditioned taste aversion to a sucrose solution (Grigson, Lyuboslavsky, Tanase, \& Wheeler, 1999). It was suggested that water deprived animals also minimise food intake effectively leaving them water and food deprived. Considering sucrose has a caloric value it is possible that continued consumption of the sucrose solution even after morphine administration may have been due to hunger. 
While the current results were produced with heroin and not morphine these two drugs have a highly similar mechanism of action and follow a similar pattern suggesting that this particular combination of parameters was sufficient to disrupt the effect. It should be noted that other research has demonstrated conditioned taste aversion to a sweet saccharin solution after the administration of morphine (Arthurs, Lin, Amodeo, \& Reilly, 2012; Fenu et al., 2010). These studies provide a useful comparison for conditioned taste aversion developed in response to either a sucrose or saccharin solution. That is, morphine paired with sucrose appears to be insufficient to induce conditioned taste aversion whereas the morphine/saccharin combination produces the predicted effect. Additional research has demonstrated that opioid agonists increase feeding behaviour (Sanger \& McCarthy, 1980); specifically opioids potentiate the natural reward that accompanies palatable foods (K. Evans \& Vaccarino, 1990). This may explain why conditioned taste aversion can be detected when morphine is paired with saccharin but not sucrose; saccharin, unlike sucrose, has no caloric value. Moreover, it may explain the difference between heroin and psychostimulant drugs (MDMA included) as the latter are appetite suppressants (Corwin, Woolverton, Schuster, \& Johanson, 1987; S. Evans \& Johanson, 1987; Griffiths, Winger, Brady, \& Snell, 1976; Wood \& Emmettoglesby, 1988). Consequently, considering the discussion given above it cannot be concluded that heroin does not produce conditioned taste aversion but depends on the type of appetitive solution that is paired with the drug. Therefore, it could be useful to evaluate heroin induced conditioned taste aversion when the drug is paired with a saccharin solution.

Considering the locomotor activity and conditioned taste aversion results together highlights a differential sensitivity between the genotypes to MDMA effects but not to those driven by heroin. Locomotor activity was facilitated for animals with intact SERT function suggesting that the WT group is more sensitive to the positive rewarding effects of MDMA. 
However, it is posited that locomotor activity for drugs like MDMA may not provide an accurate measure of the rewarding properties of these particular drugs. Therefore, from these experiments it cannot be concluded that subjects with reduced SERT function are more (or less) sensitive to the positive effects of drugs of abuse. Interestingly, the current data does suggest that subjects with reduced SERT function may be more sensitive to the negative effects of MDMA considering HOM animals showed the greatest conditioned taste aversion of the three groups. Moving forward it would be very useful to further evaluate these specific drug effects across the genotypes.

This chapter highlighted differences in genotype when positive and negative drug effects were evaluated systematically. However, in attempting to understand the complex phenomena of drug addiction is it essential to evaluate changes in behaviour as well as changes in brain function. Through novelty suppressed feeding, drug self-administration, locomotor activity and conditioned taste aversion Chapters 2 and 3 have sought to understand how reduced SERT function interacts with different types of drugs and influences different types of behaviour. Moving forward, in Chapter 4, is a discussion regarding changes in the brain as a result of drug taking (MDMA or heroin). This represents a very useful neurobiological correlate to the behavioural data that has been presented thus far. 


\section{Chapter 4}

\section{Quantitative Reverse Transcription Polymerase Chain Reaction}

\subsection{Introduction}

Drug addiction, or substance use disorder, is technically defined in terms of behavioural changes in the individual. However, there is a huge body of research to support the idea that drug addiction facilitates long term changes in the way the brain functions. As discussed earlier researchers have hypothesised that drug addiction represents changes in synaptic plasticity (i.e. learning); where neural connections in the dopaminergic system become inflexible leading to habitual drug intake.

While synaptic plasticity encompasses a variety of processes in different structures throughout the brain a family of proteins, neurotrophic factors, play an important role in the synaptic plasticity that underlies learning and memory (Binder \& Scharfman, 2004). 


\section{Brain Derived Neurotrophic Factor (BDNF)}

One protein of interest is brain derived neurotrophic factor (BDNF); as a member of the neurotrophic factor family it influences the development of the brain by promoting the survival and growth of neurons. Additionally, BDNF has been implicated in synaptic transmission, neurogenesis and importantly, the synaptic plasticity that coincides with learning (Binder \& Scharfman, 2004; Conner et al., 1997). Found widely throughout the central nervous system BDNF binds to the tropomyosin related kinase $B$ receptor (trkB).

\section{BDNF and Neuropsychiatric Illness}

BDNF plays a crucial role in neurological basis of psychiatric illnesses like depression. Research has demonstrated that decreased BDNF expression coincides with depression and stress (Igncio, Rus, Abelaira, \& Quevedo, 2014). Antidepressants have been shown to be effective in eliminating these effects; over a course of treatment BDNF levels return to normal. Importantly, many current antidepressants, with differing mechanisms of action, have been shown to increase BDNF levels; these studies provide strong evidence that regulating levels of BDNF is important for the treatment of depression (Duman \& Monteggia, 2006; Kozisek et al., 2008). It should be noted that these previous studies have utilised normal animals; therefore, additional research using the SERT KO rat was conducted to determine the influence of a compromised serotonergic system on BDNF expression. Specifically, in rodent studies researchers have demonstrated that a reduction in the SERT may influence baseline mRNA levels of total BDNF; compared to WT, HOM rats showed significantly reduced total BDNF mRNA expression in both the hippocampus and prefrontal cortex. Furthermore, studies have investigated changes in baseline mRNA levels of specic coding regions (isoforms) in the BDNF gene. mRNA levels of BDNF isoforms III, IV, VI and IXa in the hippocampus were reduced in 
HOM rats compared to WT; BDNF isoforms IV and VI were also reduced in the prefrontal cortex in HOM animals (Molteni et al., 2010). Additional research has investigated changes in BDNF expression after the administration of the antidepressant, duloxetine, which targets the SERT and the norepinephrine transporter (NET) prolonging 5-HT and norepinephrine action in the synapse. After treating HOM and WT animals they found that in both genotypes total BDNF mRNA levels were increased in the hippocampus, the prefrontal cortex and the frontal cortex. They also investigated changes in specific BDNF isoforms and found that BDNF III and BDNF IXa mRNA levels were increased in the hippocampus in both genotypes. Interestingly, they also saw increased mRNA levels in the prefrontal cortex and hippocampus for BDNF transcripts IV and VI in the HOM animals but not the WT. Taken together duloxetine treatment restored BDNF mRNA levels in HOM animals and increased the normal levels of BDNF mRNA in the WT (Calabrese et al., 2010).

Whereas decreased levels of BDNF contribute to the neurological changes associated with depression there is evidence to suggest that increased BDNF may be linked to drug addiction. For example, several research groups have demonstrated that BDNF mRNA levels in rats were increased in several brain structures (e.g. striatum, nucleus accumbens) after a single injection of cocaine (Berglind et al., 2007; Le Foll et al., 2005). Another study showed the same effect after a single dose of MDMA was administered (Martinez-Turrillas et al., 2006). Subsequent research has determined that when BDNF levels were increased in rats so did their self-administration of cocaine (Graham et al., 2007; McGinty et al., 2010). Interestingly, research has demonstrated that opioid (morphine) exposure initially decreases BDNF expression in addition to reducing the physical size of dopamine neurons in the ventral tegmental area (Chu et al., 2007; Sklair-Tavron et al., 1996). Other studies investigating the role of BDNF in heroin addiction demonstrated that BDNF mRNA was not influenced after heroin exposure (Theberge et al., 2011, 2012). However, increases in total BDNF 
expression in the medial prefrontal cortex after withdrawal from chronic opioid exposure have also been demonstrated (Kuntz-Melcavage, Brucklacher, Grigson, Freeman, \& Vrana, 2009). Additionally, another study revealed that BDNF mRNA and BDNF protein levels in the ventral tegmental area were significantly increased in rats experiencing withdrawal from heroin (Vargas-Perez et al., 2009). Similarly, Grimm et al. (2003) demonstrated increased BDNF protein levels in the mesolimbic dopamine pathway (e.g. ventral tegmental area) after withdrawal from cocaine (Grimm et al., 2003). More recently, a study investigating withdrawal from methamphetamine showed that BDNF mRNA levels are significantly increased in the dorsal striatum (X. Li et al., 2013). The same group also demonstrated that cocaine withdrawal influenced BDNF expression in the nucleus accumbens; specifically, BDNF expression in the core increased over days while the shell only showed increases after withdrawal day 90 (X. Li et al., 2015). These studies suggest a specific role for BDNF in the incubation of craving for sevearl different drugs of abuse.

Regarding specific BDNF isoforms studies have demonstrated that treatment with cocaine can lead to differential changes in mRNA levels across isoforms. For example, Liu et al. (2006) demonstrated that mRNA levels of BDNF isoform IV were significantly increased in the striatum after cocaine administration (Liu et al., 2006). Additionally, research has shown that cocaine abstinence facilitates brain region specific increases in mRNA levels of BDNF isoforms (e.g. increased BDNF isoform IV in the medial prefrontal cortex) (Sadri-Vakili et al., 2010). Furthermore, a follow up study demonstrated that forced cocaine abstinence resulted in isoform specific fluctuations in the ventral tegmental area where BDNF transcript I was increased whereas BDNF transcript IV was unaffected (H. D. Schmidt et al., 2012). These studies suggest a specific role for BDNF in the neurological changes that accompany drug addiction. Dopaminergic pathways project to a variety of brains regions (e.g. frontal cortex and striatum); considering these neurons are influenced by drugs of abuse it would be useful to eval- 
uate BDNF changes in the structures where they terminate. Therefore, the current set of experiments measured changes in total BDNF and individual BDNF isoforms (III, IV, VI, and IXa) in the frontal cortex and striatum to evaluate the specific drug induced neuroadaptations that occur in subjects with altered serotonergic function.

\subsubsection{Predictions}

Prediction 1: Considering drugs of abuse like cocaine increase BDNF mRNA levels it is predicted that MDMA treatment will produce an increase in total BDNF mRNA levels and this increase will be inversely related to SERT function $(\mathrm{HOM}>\mathrm{HET}>\mathrm{WT})$.

Prediction 2: Considering that cocaine differentially influences BDNF isoforms it is predicted that MDMA treatment will produce an increase in BDNF isoform IV mRNA levels and this increase will be inversely related to SERT function $(\mathrm{HOM}>\mathrm{HET}>\mathrm{WT})$.

Prediction 3: Considering that heroin exposure not does appear to influence BDNF expression it is predicted that heroin treatment will not produce an overall change in total BDNF mRNA levels nor individual BDNF isoforms (III, IV, VI and IXa) and this will not differ between HOM, HET, and WT groups.

\subsection{Methods}

\subsubsection{Quantitative Reverse Transcription Polymerase Chain Reaction}

At the conclusion of the MDMA and heroin self-administration experiments animals were sacrificed and brain tissue was extracted. For comparison untreated, matched groups of animals from each genotype were 
included and are designated "clean" or "untreated" below. Selected tissue sections, the frontal cortex and striatum, were excised; the entire striatum was taken for this experiment due to the small size of individual subsections (e.g. ventral, dorsal, dorsomedial or dorsolateral). Relative mRNA expression was determined via Quantitative Reverse Transcription Polymerase Chain Reaction (qRT-PCR). All procedures described below were carried out in a dedicated facility with precision qRT-PCR equipment.

In short, RNA was rapidly extracted and reverse transcribed into complimentary DNA (cDNA). cDNA was amplified during the qPCR phase and amplifications were visualised in real time by intercalation of the dye SYBR Green that shows high fluorescence when bound to double stranded DNA (and minimal fluorescence when free in solution). In a high efficiency reaction the qPCR products double with each cycle during the exponential phase of amplification. Cycle threshold values $\left(C_{\mathrm{T}}\right)$ for each sample correspond to the cycle number at which the accumulated amplification products surpass the threshold for detection. Typically, the detection threshold is automatically set by Bio-Rad software to be approximately 10 standard deviations above the baseline fluorescence value (Nolan, Hands, \& Bustin, 2006).

\section{Tissue Dissection}

Manipulations were undertaken at $4{ }^{\circ} \mathrm{C}$ or below and all tubes, pipette tips and pestles were autoclaved to prevent nuclease degradation of the RNA. Frozen tissue was sliced into $1.5 \mathrm{~mm}$ sections using a chilled aluminium brain cutting block according to a method described previously (Heffner, Hartman, \& Seiden, 1980). Each section was placed on a glass dish set in ice where the frontal cortex and striatum were rapidly dissected and placed individually into sterile $1.5 \mathrm{ml}$ Eppendorf tubes. TRIzol reagent was pipetted into each tube as per the manufacturer's instructions: $1 \mathrm{ml}$ TRIzol reagent per 50-100 mg of tissue. Individual samples weighed 
less than $50 \mathrm{mg}$; therefore, $0.5 \mathrm{ml}$ TRIzol reagent was added to each tube. With a sterile plastic pestle each sample was immediately homogenised and then frozen at $-80{ }^{\circ} \mathrm{C}$ until further use. The analysis below describes changes in the striatal tissue only.

\section{RNA Extraction}

As described in Sargeant (2008) samples stored in TRIzol were thawed and centrifuged at $13,000 \times \mathrm{g}$ at $4{ }^{\circ} \mathrm{C}$ for $15 \mathrm{~min}$ to remove cell debris. The supernatant was transferred to a new micro centrifuge tube and the RNA was extracted from the TRIzol solution as described in the manufacturer's instructions. Briefly, chloroform $(50 \mu \mathrm{l})$ was added to the $0.5 \mathrm{ml}$ TRIzol extract and centrifuged at $13000 \mathrm{x}$ for $10 \mathrm{~min}$ to recover the upper aqueous phase containing the RNA. The aqueous phase was transferred to a new micro centrifuge tube where it was mixed with 0.5 volumes of absolute ethanol. A modification to the TRIzol extraction instructions was utilised where additional purification was achieved with an RNA Isolation Kit (GeneJet RNA Cleanup and Concentration Micro Kit, ISO9001/ISO14001, K0842). In short, the aqueous TRIzol phase was mixed with equal volumes of absolute ethanol and applied to a micro centrifuge column. Under high ethanol concentration RNA binds to the column where it can be washed and eluted by following the manufacturer's instructions. The column was washed with supplied wash buffers (wash buffer 1, 00115362; wash buffer 2, 00115356, prepared as directed). Each wash was achieved by applying a volume of $700 \mu \mathrm{l}$ and centrifuging for 1 minute at 14,000 x g. The RNA was then eluted in $12 \mu \mathrm{l}$ of $\mathrm{ddH}_{2} \mathrm{O}$ and captured in a new micro centrifuge tube. RNA yield, quantity and quality were assessed by UV spectroscopy using a ND-1000 “Nanodrop” spectrophotometer (Nanodrop, Wilmington, DE) to record A260, A280 and A230 absorbance. A A260/280 absorbance ratio for each sample was calculated where $\sim 2.0$ is the accepted standard for "pure" RNA (Sargeant, 2008). 


\section{Reverse Transcription}

Reverse transcriptions were performed using the Superscript III kit and components (Invitrogen, 18080-044). Reactions were prepared in two stage$\mathrm{s}$ (see Table 4.1). First, Oligo $\mathrm{dT}_{20}$, RNA, deoxynucleotides (dNTPs) and $\mathrm{ddH}_{2} \mathrm{O}$ were added to achieve a total volume of $26 \mu \mathrm{l}$ and the mixture was heated to $65{ }^{\circ} \mathrm{C}$ for 5 minutes then snap chilled. Second, the following components were added: 5x First-Strand Buffer, dithiothreitol (DTT), RNaseOUT and Superscript III to a total overall volume of $40 \mu \mathrm{l}$. The RT reaction was heated in a water bath at $50{ }^{\circ} \mathrm{C}$ for 60 minutes and terminated by heating to $75^{\circ} \mathrm{C}$ for 15 minutes.

\begin{tabular}{|l|r|r|}
\hline \multicolumn{3}{|r|}{ Stage 1 } \\
\hline Component & Concentration & Volume \\
\hline Oligo dT $_{20}$ & $2.5 \mu \mathrm{M}$ & $2 \mu \mathrm{l}$ \\
\hline dNTP & $10 \mu \mathrm{M}$ & $2 \mu \mathrm{l}$ \\
\hline RNA & $1-3 \mu \mathrm{g}$ & $7.5 \mu \mathrm{l}$ \\
\hline ddH $_{\mathbf{2}} \mathbf{O}$ & - & $14.5 \mu \mathrm{l}$ \\
\hline \hline \multicolumn{3}{|c|}{ Stage 2 } \\
\hline Component & Concentration & Volume \\
\hline DTT & $0.1 \mathrm{M}$ & $2 \mu \mathrm{l}$ \\
\hline RNaseOUT & $40 \mathrm{U} / \mu \mathrm{l}$ & $2 \mu \mathrm{l}$ \\
\hline Superscript III & $200 \mathrm{U} / \mu \mathrm{l}$ & $2 \mu \mathrm{l}$ \\
\hline 5x First-Strand Buffer & $250 \mathrm{mM} \mathrm{Tris-HCl}$ & $8 \mu \mathrm{l}$ \\
& $375 \mathrm{mM} \mathrm{KCl}$ & \\
\hline
\end{tabular}

Table 4.1: Reverse transcription reaction information: Component concentrations and volumes. 


\section{Quantitative Reverse Transcription Polymerase Chain Reaction}

Amplifications were performed in triplicate in final volume of $25 \mu \mathrm{l}$. Master mixes containing Platinum SYBR Green qRT-PCR SuperMix-UDG (2x concentration, SMPF-212G), cDNA (1 $\mu$ l of the heat activated RT mixture) and water were prepared and added to each primer set $(0.2 \mu \mathrm{M}$ forward and reverse primers indicated in Table 4.2).

Control reactions were included with each amplification. A no reverse transcription control (NRT) was prepared for each sample where the cDNA template was replaced with sample RNA; amplification in a NRT reaction would indicate the presence of contaminating genomic DNA in the sample. Additionally, a no template control (NTC) was prepared where the cDNA template was replaced with water (one per 96 well plate); amplification in a NTC reaction may indicate contamination during the qRT-PCR setup (e.g. pipette tips, tubes).

qRT-PCR cycling conditions were as previously described (Calabrese et al., 2010; Molteni et al., 2009). Samples were heated to $50{ }^{\circ} \mathrm{C}$ for $2 \mathrm{~min}$ then to $95^{\circ} \mathrm{C}$ for $10 \mathrm{~min}$ followed by 40 cycles of denaturation at $95^{\circ} \mathrm{C}(15$ s) and annealing/extension at $60{ }^{\circ} \mathrm{C}(1 \mathrm{~min})$. An example of exponential product amplification for three representative samples is given in Figure 4.1. Melt curve analysis was conducted between 65 and $95{ }^{\circ} \mathrm{C}$ to confirm correct amplification of qPCR products. An example of three representative samples is given in Figure 4.2.

\section{Data Analysis}

Five BNDF targets were evaluated: total BDNF, BDNF transcript III, BDNF transcript IV, BDNF transcript VI and BDNF transcript IXa (see Table 4.2). Expression relative to two housekeeping genes, Cyclophilin A (Cyc A) and Hypoxanthine-guanine phosphoribosyltransferase (HPRT), was undertaken. These two housekeeping genes have been used extensively in similar studies of mRNA expression analysis of brain tissue (Aid, Kazant- 


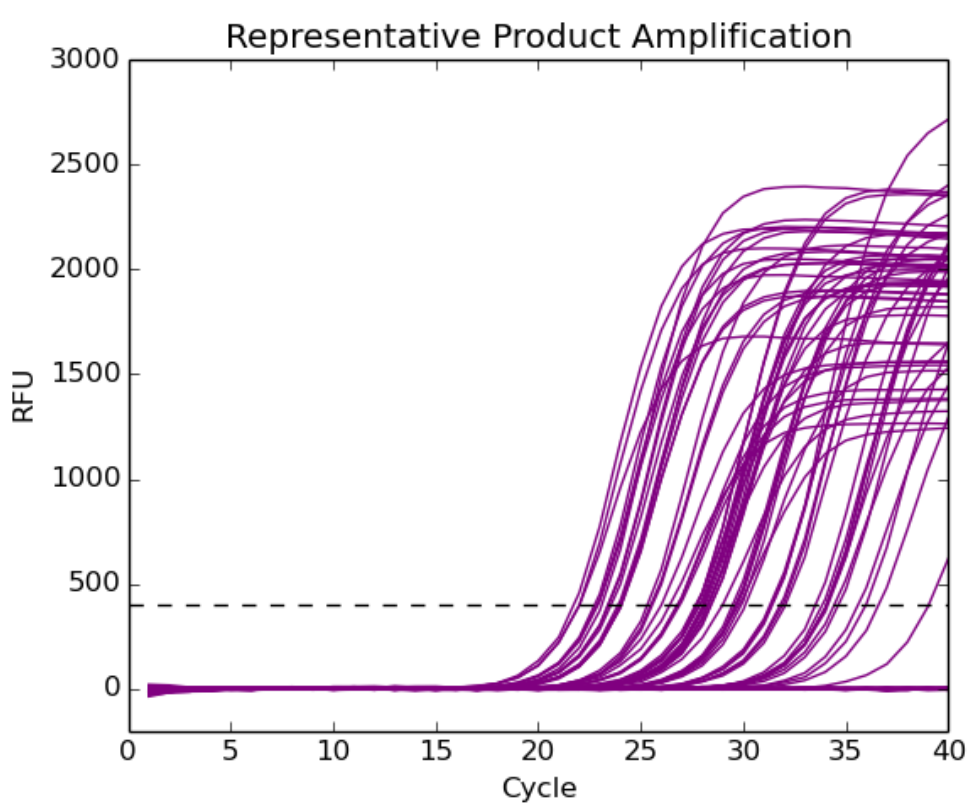

Figure 4.1: Representative qRT-PCR amplification of mRNA from the striatum of heroin treated subjects. Amplification targets included: $\mathrm{Cy}-$ clophilin A, HPRT, Total BDNF, and BDNF trasncripts III, IV, VI and IXa. The detection threshold is indicated by the dash line.

seva, Piirsoo, Palm, \& Timmusk, 2007; Sargeant, 2008). As internal control genes their expression should not vary significantly between genotypes or treatments. All amplification triplicates were scrutinised and a pattern emerged: the first triplicate consistently differed from the second and third. As this occured across all samples the second and third triplicate were averaged and relative gene expression was calculated using the comparative cycle threshold $\left(C_{\mathrm{T}}\right)$ method. For each sample the cycle threshold value for the housekeeping gene was subtracted from the sample threshold cycle $\left(\Delta C_{\mathrm{T}}=C_{\mathrm{T}}\right.$ target gene $-C_{\mathrm{T}}$ housekeeping gene). Then each $\Delta C_{\mathrm{T}}$ value was converted into the negative binary $\log \left(2^{-\Delta C_{T}}\right)$ prior to statistical analysis. When the sample threshold cycle exceeded the housekeeping gene threshold cycle the resulting $2^{-\Delta C_{\mathrm{T}}}$ value would be $<1$ indicating a 


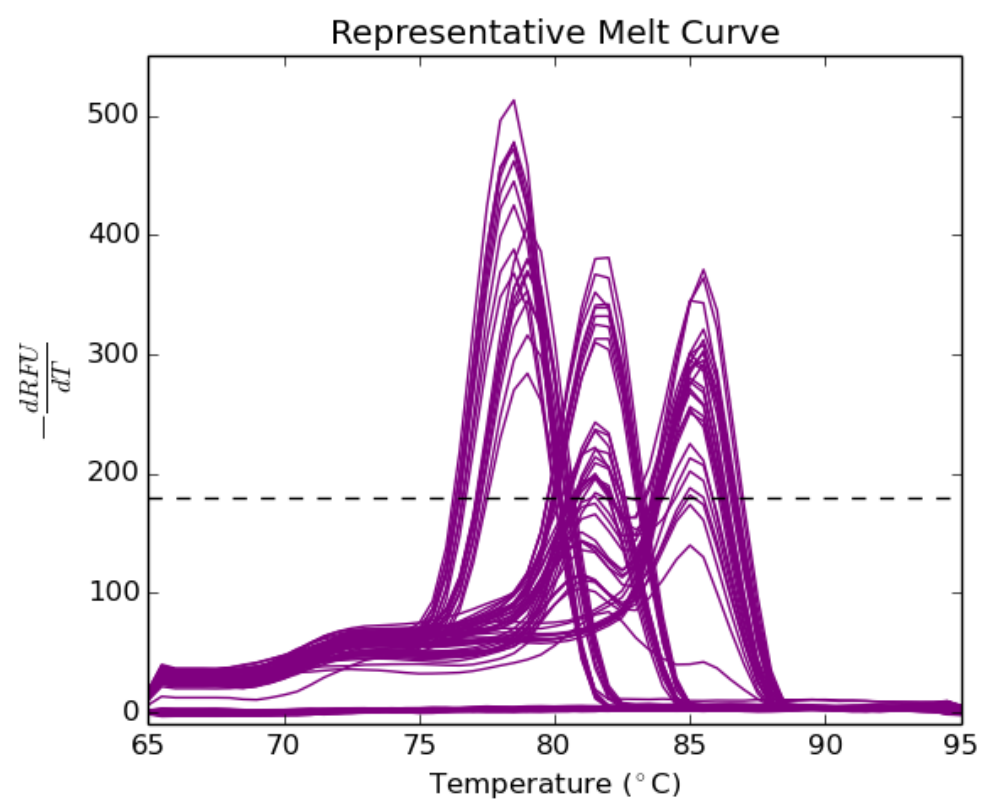

Figure 4.2: Representative melt curve analysis with a peak for each amplicon consistent with the predicted PCR temperature. Amplification targets and melting temperatures are as follows: Cyclophilin A $\left(81.5^{\circ} \mathrm{C}\right), \mathrm{HPRT}$ $\left(79.5^{\circ} \mathrm{C}\right)$, Total BDNF $\left(82^{\circ} \mathrm{C}\right)$, BDNF III $\left(79^{\circ} \mathrm{C}\right)$, BDNF IV $\left(81.5^{\circ} \mathrm{C}\right)$, BDNF VI $\left(85.5^{\circ} \mathrm{C}\right)$ and BDNF IXa $\left(85^{\circ} \mathrm{C}\right)$. The threshold filter is indicated by the dash line.

reduction in gene expression; therefore, by taking the negative inverse of the binary $\log \left(-1 / 2^{-\Delta C_{\mathrm{T}}}\right)$ yielded a fold change reduction for the given sample (Schmittgen \& Livak, 2008).

It should be noted that while evaluating changes in gene expression by analysing $\Delta C_{\mathrm{T}}$ values is common practice it may not represent the most appropriate method. This is due to the fact that a qRT-PCR reaction running at optimal efficiency (100\%) will, with each cycle, produce a doubling the of the amplification products. Therefore, small $\Delta C_{\mathrm{T}}$ values may be deemed insignificant when compared via statistical analysis that does not take into account the exponential nature of the data points. Additionally, 


\begin{tabular}{|l|r|}
\hline \multicolumn{2}{|r|}{ Total BDNF } \\
\hline Forward & AGCTGAGCGTGTGTGACAGT \\
\hline Reverse & ACCCATGGGATTACACTTGG \\
\hline Reference & Musazzi et al. 2009 \\
\hline \hline \multicolumn{2}{|c|}{ BDNF transcript III } \\
\hline Forward & ATGCTTCATTGAGCCCAGTT \\
\hline Reverse & GTGGACGTTTGCTTCTTTCA \\
\hline Reference & Calabrese et al. 2010 \\
\hline \hline \multicolumn{2}{|c|}{ BDNF transcript IV } \\
\hline Forward & TGCGAGTATTACCTCCGCCAT \\
\hline Reverse & TCACGTGCTCAAAAGTGTCAG \\
\hline Reference & Lubin et al. 2008 \\
\hline \hline \multicolumn{2}{|c|}{ BDNF transcript VI } \\
\hline Forward & TTGGGGCAGACGAGAAAGCGC \\
\hline Reverse & TCACGTGCTCAAAAGTGTCAG \\
\hline Reference & Lubin et al. 2008 \\
\hline \hline \multicolumn{2}{|c|}{ BDNF transcript IXa } \\
\hline Forward & TGGTGTCCCCAAGAAAGTAA \\
\hline Reverse & CACGTGCTCAAAAGTGTCAG \\
\hline Reference & Calabrese et al. 2010 \\
\hline
\end{tabular}

Table 4.2: Primer sequences used for qRT-PCR analysis. 
it is difficult to interpret varying $\Delta C_{\mathrm{T}}$ values because they fail to describe the actual amount of mRNA initially present in the reaction. For example, if there is a relatively small amount of input mRNA in the qRT-PCR reaction it will require additional amplification cycles to generate sufficient product to be detected for both the housekeeping gene as well as the target gene. So while two samples may yield the same $\Delta C_{\mathrm{T}}$ value they may represent vastly different amounts of amplification products. This can be problematic with particularly high $C_{\mathrm{T}}$ values (those approaching the maximum cycle number, 40) as experimentally generated noise can become significant. Such amplification has detectable but not quantifiable amounts of the template. Therefore, in an effort to circumvent these issues that surround the analysis and interpretation of $\Delta C_{T}$ values there is growing support for the use of converted negative binary log values $\left(2^{-\Delta C_{\mathrm{T}}}\right)$ in qRT-PCR statistical analysis (described above). These values represent increases or decreases in gene expression and more transparently reflect the fold changes that can be obscured when interpreting $\Delta C_{\mathrm{T}}$ values. For these reasons the analysis presented in this chapter utilises converted negative binary $\log$ values $\left(2^{-\Delta C_{T}}\right)$. Please note that for direct comparison the raw data, with accompanying figures, for both $\Delta C_{\mathrm{T}}$ and $2^{-\Delta C_{\mathrm{T}}}$ values are provided in the Appendix: Supplemental qRT-PCR Analysis. 


\subsection{Results}

\subsubsection{Average Housekeeping Genes}

To evaluate the relative expression of the housekeeping genes across genotypes and treatment groups $\Delta C_{T}$ values were calculated for each sample $\left(\Delta C_{\mathrm{T}}=C_{\mathrm{T}}\right.$ HPRT $-C_{\mathrm{T}}$ Cyc A); then each $\Delta C_{\mathrm{T}}$ value was converted into the negative binary $\log \left(2^{-\Delta C_{\mathrm{T}}}\right)$ and analysed via two way ANOVA with genotype and treatment as between-subjects variables (raw data are provided in the Appendix: Section 1). Analysis revealed a significant main effect of treatment, $F(2,35)=3.73, p=.03$; however, neither the main effect of genotype, $F(2,35)=1.65, p=.21$, nor the treatment ${ }^{*}$ genotype interaction reached significance, $F(4,35)=.59, p=.67$ (see Figure 4.3).

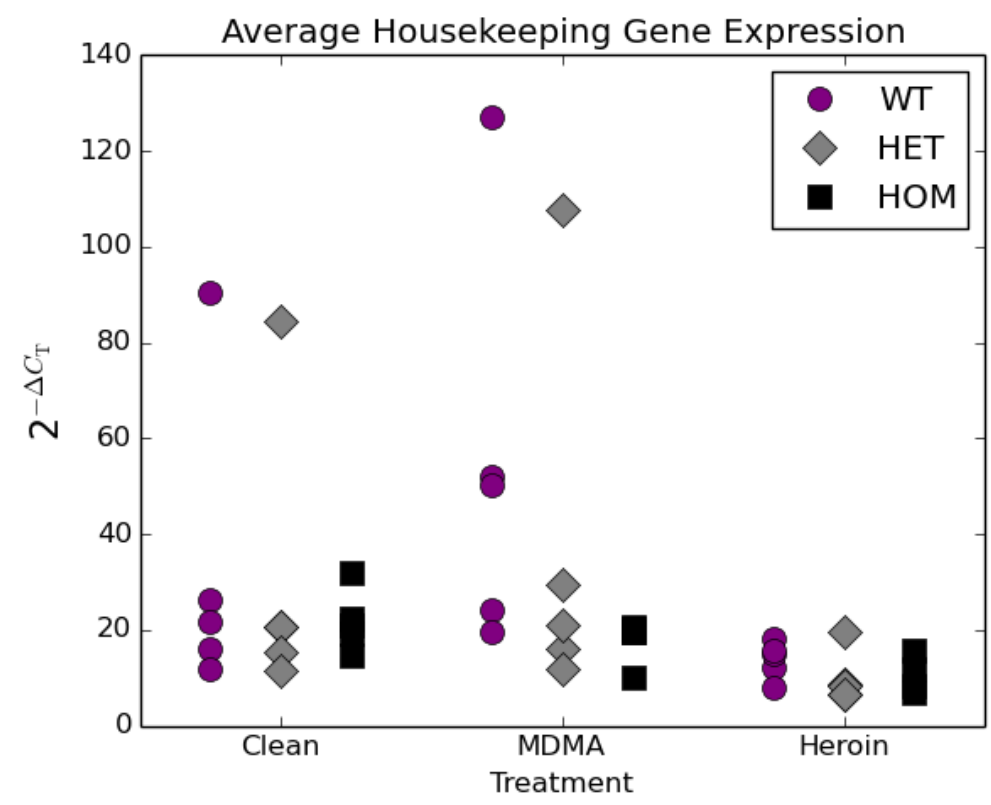

Figure 4.3: Relative housekeeping gene expression across treatment groups and genotypes.

Post hoc analysis was undertaken and it revealed a significant differ- 
ence within the HOM group between heroin treated and untreated (clean) subjects; heroin treated subjects showed lower $2^{-\Delta C_{\mathrm{T}}}$ values compared to the untreated (clean). Considering this effect was only seen in HOM subjects under one specific treatment regimen it is unlikely that this variation represents a confounding variable; that is, the effect does not nullify the selected housekeeping genes as internal references for these experiments. Therefore, the five BDNF targets listed in Table 4.2 were compared to both Cyclophilin A and HPRT.

\subsubsection{Total BDNF}

\section{Cyclophilin A}

To evaluate relative gene expression of total BDNF (mRNA) $\Delta C_{\mathrm{T}}$ values were calculated for each sample $\left(\Delta C_{T}=\left(C_{T}\right.\right.$ total BDNF $)-\left(C_{T}\right.$ Cyc $\left.\left.A\right)\right)$. Then each $\Delta C_{T}$ value was converted into the negative binary $\log \left(2^{-\Delta C_{T}}\right)$. Individual datum values are provided in the Appendix: Section 2. Data were analysed via two way ANOVA with genotype and treatment as between-subjects variables. Comparing clean and MDMA treated samples revealed a significant main effect of treatment, $F(1,24)=19.91, p<.001$, with MDMA treated samples showing higher converted $2^{-\Delta C_{\mathrm{T}}}$ values compared to the clean group. The main effect of genotype and the treatment ${ }^{*}$ genotype interaction failed to reach significance $F(2,24)=1.11, p=.35$ and $F(2,24)=$ $1.97, p=.16$, respectively. Conversely, comparing clean and heroin treated samples did not reveal a significant main effect of treatment, $F(1,23)=.15$, $p=.7$, nor genotype, $F(2,23)=.001, p=1.0$, and the treatment ${ }^{*}$ genotype interaction failed to reach significance $F(2,23)=.18 p=.83$, (see Figure 4.4).

\section{HPRT}

To evaluate relative gene expression of total BDNF (mRNA) $\Delta C_{T}$ values were calculated for each sample $\left(\Delta C_{T}=\left(C_{T}\right.\right.$ total BDNF $)-\left(C_{T}\right.$ HPRT $\left.)\right)$. Then each $\Delta C_{T}$ value was converted into the negative binary $\log \left(2^{-\Delta C_{T}}\right)$. 


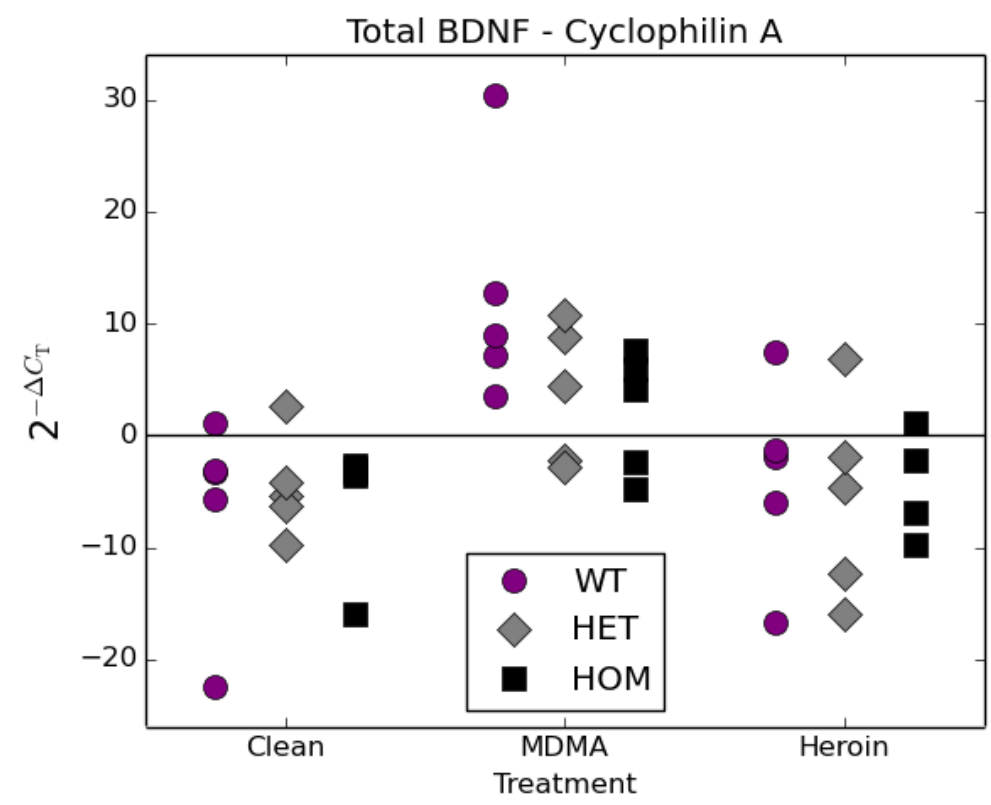

Figure 4.4: Converted $2^{-\Delta C_{\mathrm{T}}}$ values for total BDNF against Cyclophilin A.

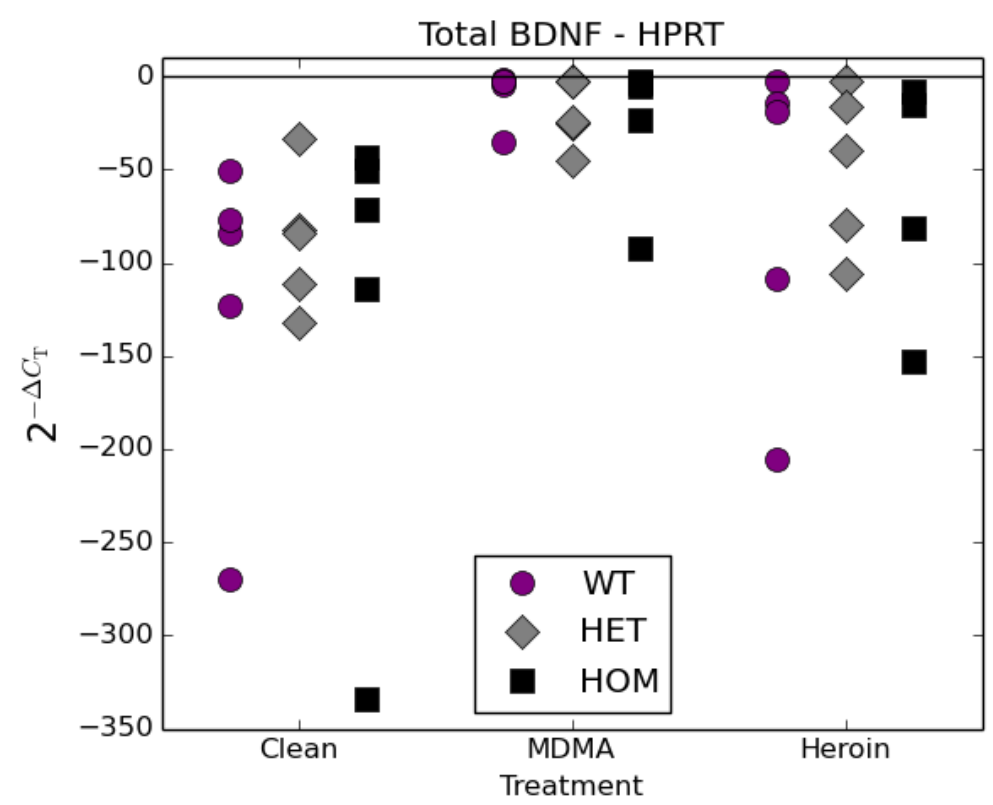

Figure 4.5: Converted $2^{-\Delta C_{\mathrm{T}}}$ values for total BDNF against HPRT. 
Individual datum values are provided in the Appendix: Section 2. Data were analysed via two way ANOVA with genotype and treatment as between-subjects variables. Comparing clean and MDMA treated samples revealed a significant main effect of treatment, $F(1,24)=14.91, p=.001$, with MDMA treated samples showing higher converted $2^{-\Delta C_{\mathrm{T}}}$ values compared to the clean group. The main effect of genotype and the treatment ${ }^{*}$ genotype interaction failed to reach significance $F(2,24)=.23, p=.8$ and $F(2,24)=$ $.28, p=.76$, respectively. Conversely, comparing clean and heroin treated samples did not reveal a significant main effect of treatment, $F(1,23)=2.79$, $p=.11$, nor genotype, $F(2,23)=.34, p=.71$, and the treatment genotype $^{*}$ interaction failed to reach significance $F(2,23)=.03, p=.97$ (see Figure 4.5).

\subsubsection{BDNF transcript III}

\section{Cyclophilin A}

To evaluate relative gene expression of BDNF transcript III (mRNA) $\Delta C_{\mathrm{T}}$ values were calculated for each sample $\left(\Delta C_{\mathrm{T}}=\left(C_{\mathrm{T}}\right.\right.$ BDNF III $)-\left(C_{\mathrm{T}}\right.$ Cyc $\mathrm{A})$ ). Then each $\Delta C_{\mathrm{T}}$ value was converted into the negative binary log $\left(2^{-\Delta C_{T}}\right)$. Individual datum values are provided in the Appendix: Section 3. Data were analysed via two way ANOVA with genotype and treatment as between-subjects variables. Comparing clean and MDMA treated samples revealed a significant main effect of treatment, $F(1,24)=6.44, p=$ .02 , with MDMA treated samples showing higher converted $2^{-\Delta C_{T}}$ values compared to the clean group. The main effect of genotype and the treatment ${ }^{*}$ genotype interaction failed to reach significance $F(2,24)=.28, p=$ .76 and $F(2,24)=.24, p=.79$, respectively. Conversely, comparing clean and heroin treated samples did not reveal a significant main effect of treatment, $F(1,23)=.78, p=.39$, nor genotype, $F(2,23)=.11, p=.9$, and the treatment ${ }^{*}$ genotype interaction failed to reach significance $F(2,23)=.29 p$ $=.75$, (see Figure 4.6). 


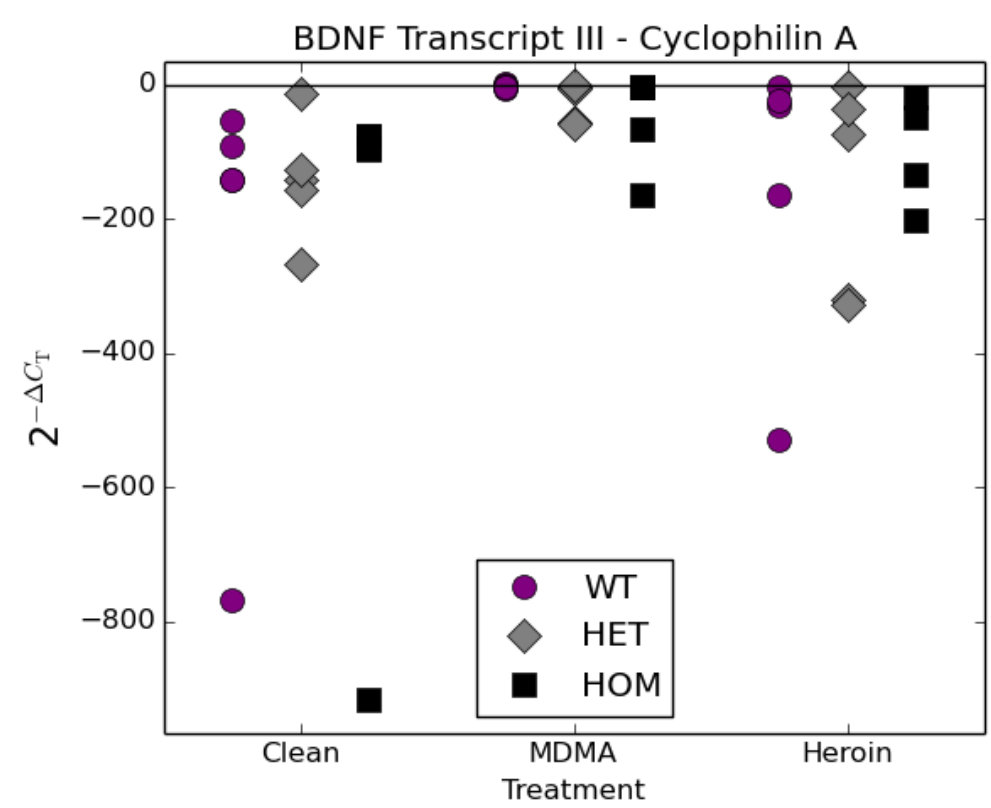

Figure 4.6: Converted $2^{-\Delta C_{\mathrm{T}}}$ values for III BDNF against Cyclophilin A.

\section{HPRT}

To evaluate relative gene expression of BDNF transcript III (mRNA) $\Delta C_{\mathrm{T}}$ values were calculated for each sample $\left(\Delta C_{\mathrm{T}}=\left(C_{\mathrm{T}}\right.\right.$ BDNF III $)-\left(C_{\mathrm{T}}\right.$ HPRT $\left.)\right)$. Then each $\Delta C_{\mathrm{T}}$ value was converted into the negative binary $\log \left(2^{-\Delta C_{\mathrm{T}}}\right)$. Individual datum values are provided in the Appendix: Section 3. Data were analysed via two way ANOVA with genotype and treatment as between-subjects variables. Comparing clean and MDMA treated samples revealed a significant main effect of treatment, $F(1,24)=8.39, p=.008$, with MDMA treated samples showing higher converted $2^{-\Delta C_{\mathrm{T}}}$ values compared to the clean group. The main effect of genotype and the treatment ${ }^{*}$ genotype interaction failed to reach significance $F(2,24)=.57, p=.57$ and $F(2,24)=$ $.43, p=.66$, respectively. Conversely, comparing clean and heroin treated samples did not reveal a significant main effect of treatment, $F(1,23)=3.63$, $p=.07$, nor genotype, $F(2,23)=.56, p=.58$, and the treatment ${ }^{*}$ genotype 
interaction failed to reach significance $F(2,23)=.30, p=.74$ (see Figure 4.7).

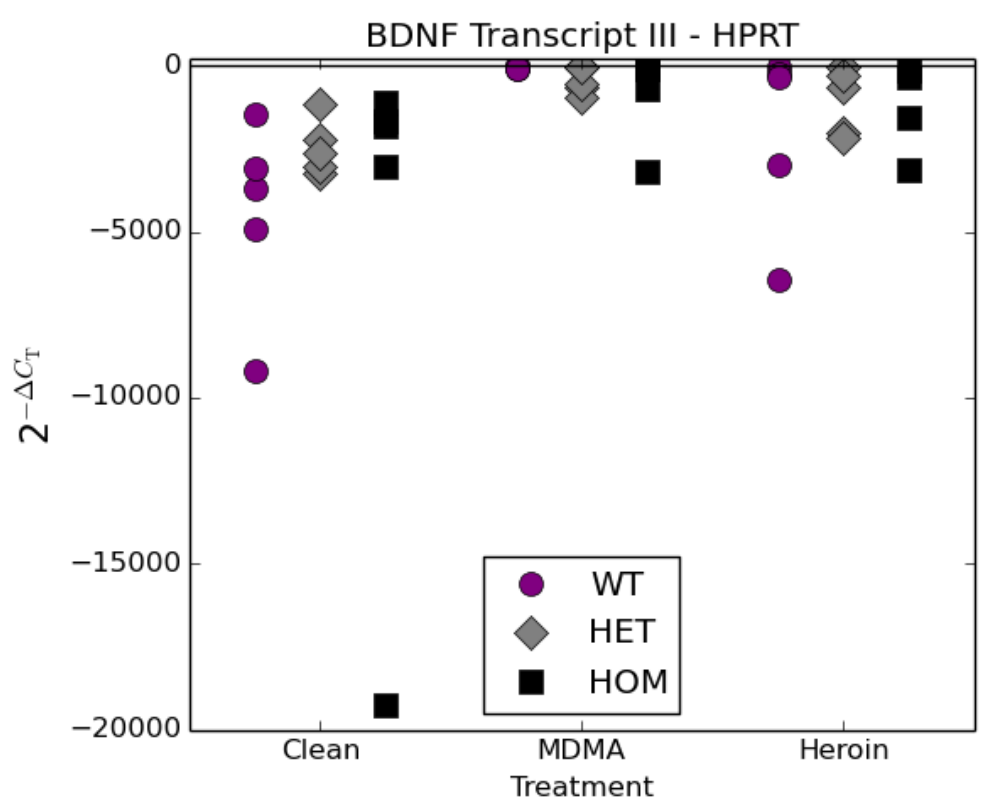

Figure 4.7: Converted $2^{-\Delta C_{\mathrm{T}}}$ values for BDNF III against HPRT.

\subsubsection{BDNF transcript IV}

\section{Cyclophilin A}

To evaluate relative gene expression of BDNF transcript IV (mRNA) $\Delta C_{T}$ values were calculated for each sample $\left(\Delta C_{T}=\left(C_{T}\right.\right.$ BDNF IV $)-\left(C_{T}\right.$ Cyc A)). Then each $\Delta C_{T}$ value was converted into the negative binary log $\left(2^{-\Delta C_{T}}\right)$. Individual datum values are provided in the Appendix: Section 4. Data were analysed via two way ANOVA with genotype and treatment as between-subjects variables. Comparing clean and MDMA treated samples revealed a significant main effect of treatment, $F(1,24)=8.93, p=$ .006 , with MDMA treated samples showing higher converted $2^{-\Delta C_{\mathrm{T}}}$ values compared to the clean group. The main effect of genotype and the treatment ${ }^{*}$ genotype interaction failed to reach significance $F(2,24)=.34, p$ 
$=.71$ and $F(2,24)=.31, p=.74$, respectively. Conversely, comparing clean and heroin treated samples did not reveal a significant main effect of treatment, $F(1,23)=.11, p=.75$, nor genotype, $F(2,23)=.11, p=.9$, and the treatment ${ }^{*}$ genotype interaction failed to reach significance $F(2,23)=.38 p$ $=.69$, (see Figure 4.8).

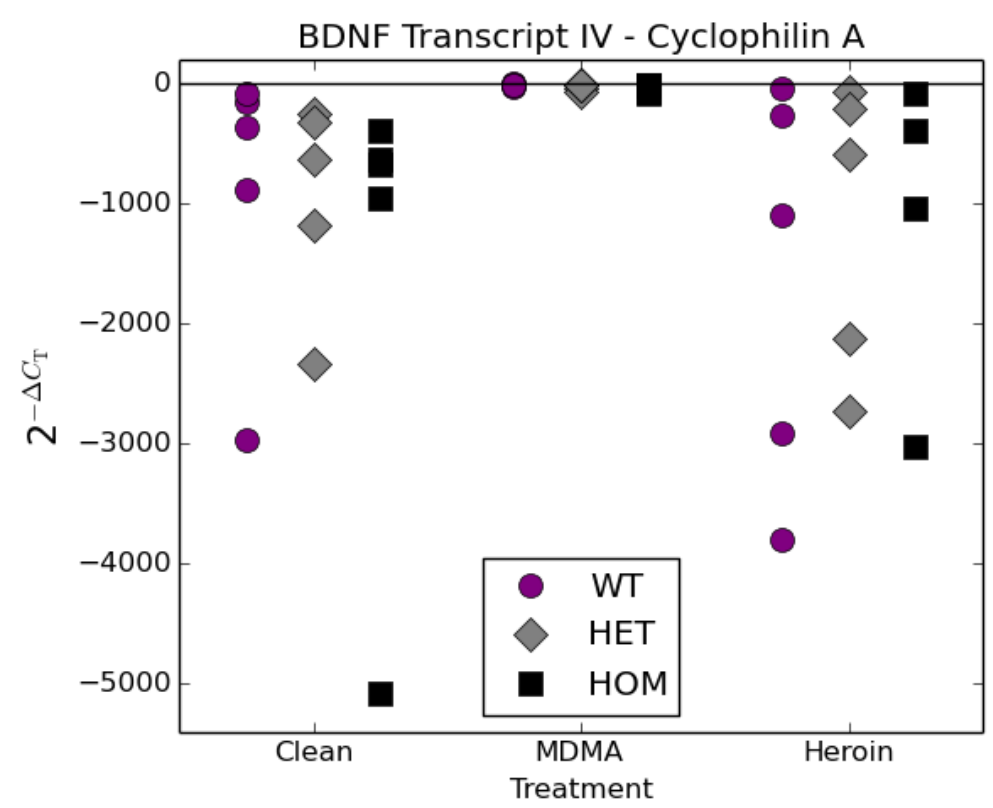

Figure 4.8: Converted $2^{-\Delta C_{\mathrm{T}}}$ values for BDNF IV against Cyclophilin A.

\section{HPRT}

To evaluate relative gene expression of BDNF transcript IV (mRNA) $\Delta C_{T}$ values were calculated for each sample $\left(\Delta C_{\mathrm{T}}=\left(C_{\mathrm{T}}\right.\right.$ BDNF IV $\left.)-\left(C_{\mathrm{T}} \mathrm{HPRT}\right)\right)$. Then each $\Delta C_{\mathrm{T}}$ value was converted into the negative binary $\log \left(2^{-\Delta C_{\mathrm{T}}}\right)$. Individual datum values are provided in the Appendix: Section 4. Data were analysed via two way ANOVA with genotype and treatment as between-subjects variables. Comparing clean and MDMA treated samples revealed a significant main effect of treatment, $F(1,24)=10.22, p=.004$, with 
MDMA treated samples showing higher converted $2^{-\Delta C_{\mathrm{T}}}$ values compared to the clean group. The main effect of genotype and the treatment ${ }^{*}$ genotype interaction failed to reach significance $F(2,24)=.57, p=.58$ and $F(2,24)=$ $.58, p=.57$, respectively. Conversely, comparing clean and heroin treated samples did not reveal a significant main effect of treatment, $F(1,23)=.84$, $p=.37$, nor genotype, $F(2,23)=.35, p=.71$, and the treatment ${ }^{*}$ genotype interaction failed to reach significance $F(2,23)=.86, p=.44$ (see Figure 4.9 ).

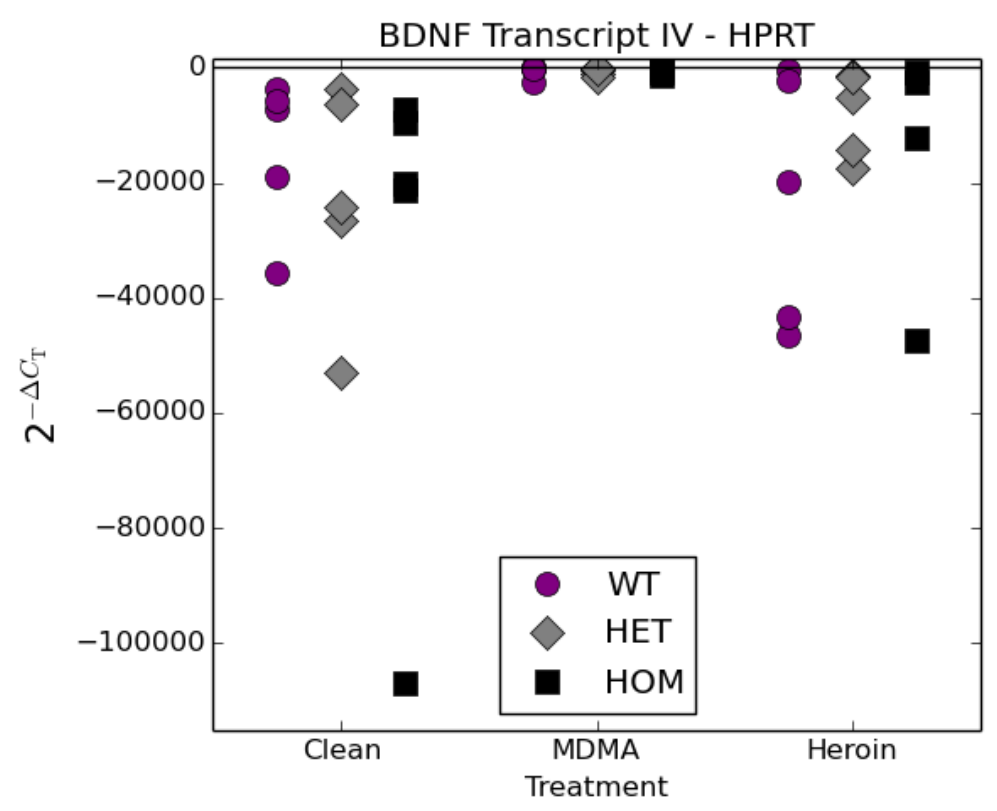

Figure 4.9: Converted $2^{-\Delta C_{\mathrm{T}}}$ values for BDNF IV against HPRT.

\subsubsection{BDNF transcript VI}

\section{Cyclophilin A}

To evaluate relative gene expression of BDNF transcript VI (mRNA) $\Delta C_{\mathrm{T}}$ values were calculated for each sample $\left(\Delta C_{T}=\left(C_{T}\right.\right.$ BDNF VI $)-\left(C_{T}\right.$ Cyc $\mathrm{A})$ ). Then each $\Delta C_{\mathrm{T}}$ value was converted into the negative binary log $\left(2^{-\Delta C_{T}}\right)$. Individual datum values are provided in the Appendix: Section 
5. Data were analysed via two way ANOVA with genotype and treatment as between-subjects variables. Comparing clean and MDMA treated samples did not reveal a significant main effect of treatment, $F(1,24)=1.41, p=$ .25 , or genotype, $F(2,24)=.38, p=.69$, and the treatment ${ }^{*}$ genotype interaction failed to reach significance $F(2,24)=.73, p=.49$. Similarly, comparing clean and heroin treated samples did not reveal a significant main effect of treatment, $F(1,23)=.001, p=.97$, or genotype, $F(2,23)=.07, p=.93$, and the treatment ${ }^{*}$ genotype interaction failed to reach significance $F(2,23)=$ $.58, p=.57$ (see Figure 4.10).

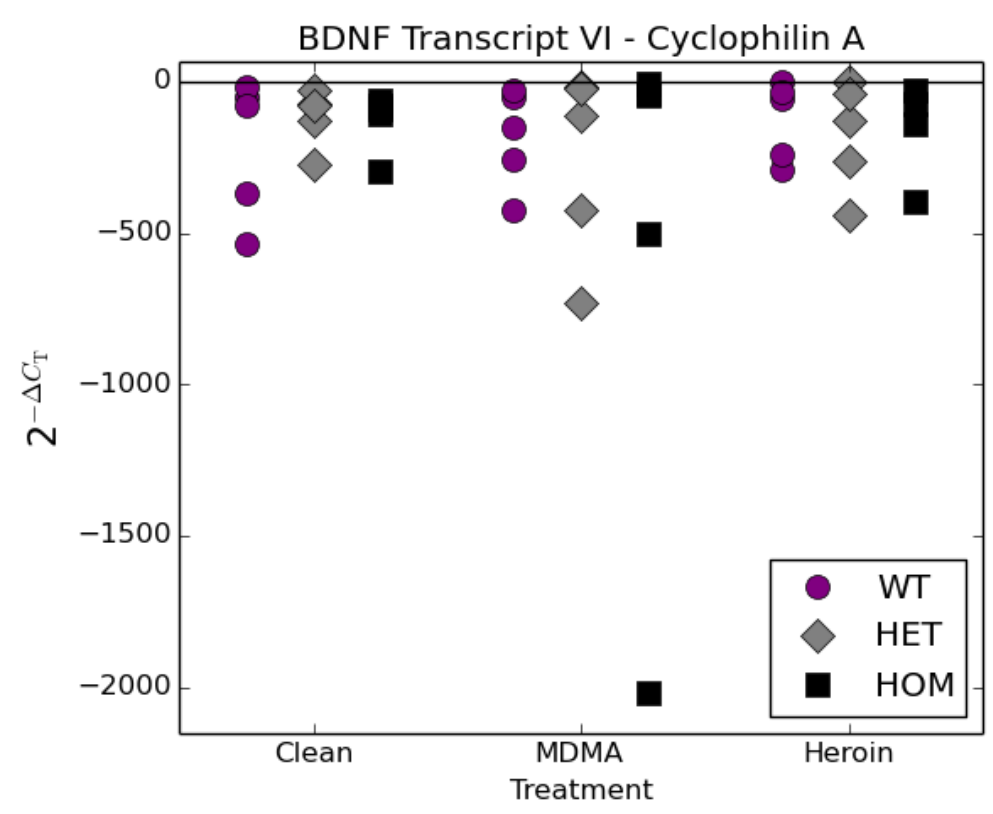

Figure 4.10: Converted $2^{-\Delta C_{\mathrm{T}}}$ values for BDNF VI against Cyclophilin A.

\section{HPRT}

To evaluate relative gene expression of BDNF transcript VI (mRNA) $\Delta C_{\mathrm{T}}$ values were calculated for each sample $\left(\Delta C_{T}=\left(C_{T}\right.\right.$ BDNF VI $)-\left(C_{T}\right.$ HPRT $\left.)\right)$. Then each $\Delta C_{\mathrm{T}}$ value was converted into the negative binary $\log \left(2^{-\Delta C_{\mathrm{T}}}\right)$. 
Individual datum values are provided in the Appendix: Section 5. Data were analysed via two way ANOVA with genotype and treatment as between-subjects variables. Comparing clean and MDMA treated samples did not reveal a significant main effect of treatment, $F(1,24)=2.85, p=.11$, or genotype, $F(2,24)=.14, p=.87$, and the treatment $t^{*}$ genotype interaction failed to reach significance $F(2,24)=.15, p=.86$. Similarly, comparing clean and heroin treated samples did not reveal a significant main effect of treatment, $F(1,23)=2.00, p=.17$, or genotype, $F(2,23)=.62, p=.55$, and the treatment ${ }^{*}$ genotype interaction failed to reach significance $F(2,23)=$ $.24, p=.79$ (see Figure 4.11).

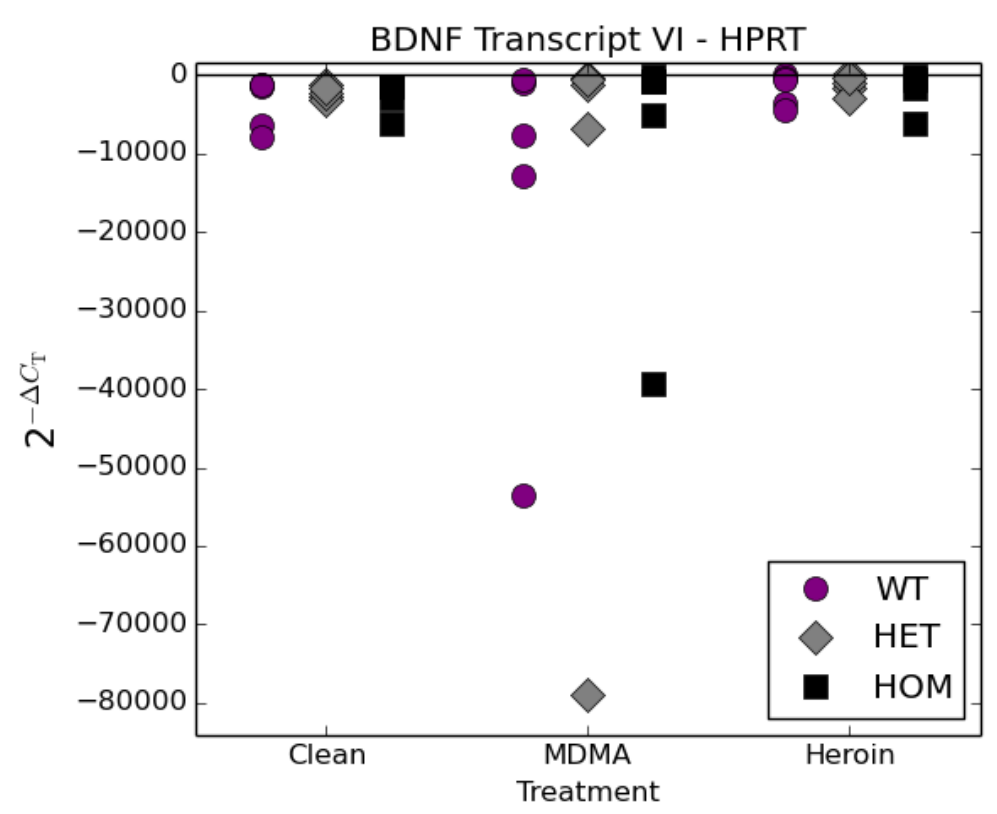

Figure 4.11: Converted $2^{-\Delta C_{\mathrm{T}}}$ values for BDNF VI against HPRT. 


\subsubsection{BDNF transcript IXa}

\section{Cyclophilin A}

To evaluate relative gene expression of BDNF transcript IXa (mRNA) $\Delta C_{T}$ values were calculated for each sample $\left(\Delta C_{T}=\left(C_{T}\right.\right.$ BDNF IXa $)-\left(C_{T}\right.$ Cyc A)). Then each $\Delta C_{T}$ value was converted into the negative binary log $\left(2^{-\Delta C_{\mathrm{T}}}\right)$. Individual datum values are provided in the Appendix: Section 6. Data were analysed via two way ANOVA with genotype and treatment as between-subjects variables. Comparing clean and MDMA treated samples did not reveal a significant main effect of treatment, $F(1,24)=2.58, p=$ .12 , or genotype, $F(2,24)=.17, p=.84$, and the treatment ${ }^{*}$ genotype interaction failed to reach significance $F(2,24)=.22, p=.80$. Similarly, comparing clean and heroin treated samples did not reveal a significant main effect of treatment, $F(1,23)=2.47, p=.13$, or genotype, $F(2,23)=.28, p=.76$, and the treatment ${ }^{*}$ genotype interaction failed to reach significance $F(2,23)=$ $.26, p=.77$ (see Figure 4.12).

\section{HPRT}

To evaluate relative gene expression of BDNF transcript IXa (mRNA) $\Delta C_{T}$ values were calculated for each sample $\left(\Delta C_{T}=\left(C_{T}\right.\right.$ BDNF IXa $)-\left(C_{T}\right.$ HPRT $\left.)\right)$. Then each $\Delta C_{\mathrm{T}}$ value was converted into the negative binary $\log \left(2^{-\Delta C_{\mathrm{T}}}\right)$. Individual datum values are provided in the Appendix: Section 6. Data were analysed via two way ANOVA with genotype and treatment as between-subjects variables. Comparing clean and MDMA treated samples did not reveal a significant main effect of treatment, $F(1,24)=1.55, p=.23$, or genotype, $F(2,24)=.44, p=.65$, and the treatment ${ }^{*}$ genotype interaction failed to reach significance $F(2,24)=.12, p=.89$. Similarly, comparing clean and heroin treated samples did not reveal a significant main effect of treatment, $F(1,23)=.1, p=.75$, or genotype, $F(2,23)=.03, p=.97$, and the treatment ${ }^{*}$ genotype interaction failed to reach significance $F(2,23)=.14, p$ $=.87$ (see Figure 4.13). 


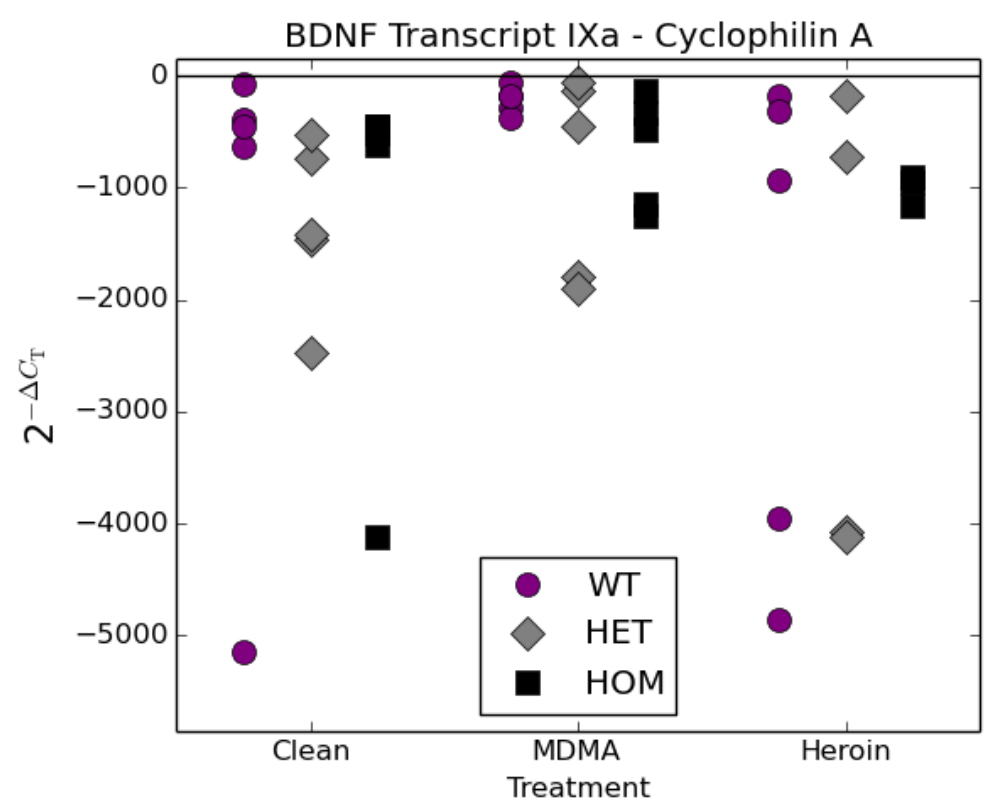

Figure 4.12: Converted $2^{-\Delta C_{\mathrm{T}}}$ values for BDNF IXa against Cyclophilin A.

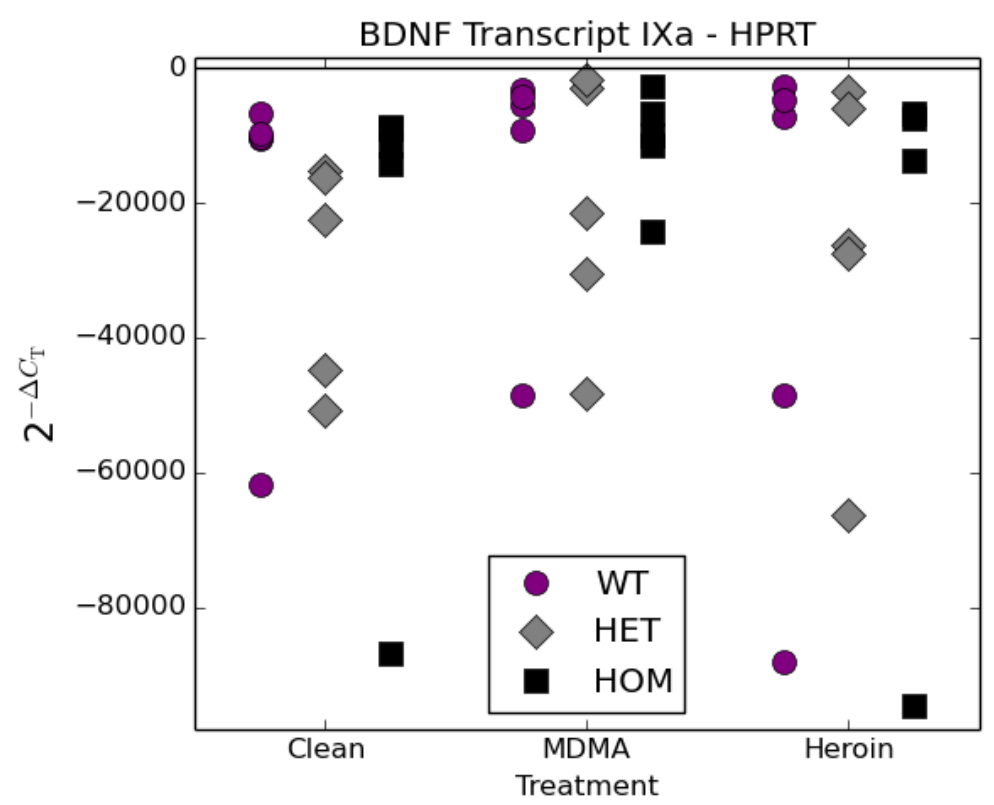

Figure 4.13: Converted $2^{-\Delta C_{\mathrm{T}}}$ values for BDNF IXa against HPRT. 


\section{Brief Summary of Results}

A significant main effect of treatment emerged for total BDNF and BDNF transcripts III and IV; that is, MDMA treatment increased relative gene expression in these targets across genotypes. Conversely, no significant differences emerged when BDNF transcripts VI and IXa were evaluated. Interestingly, heroin treatment failed to elicit a change in BDNF target expression compared to subjects that were untreated (clean).

\subsection{Discussion}

Baseline levels of Cyclophilin A and HPRT were compared to each other to evaluate relative differences in expression between the two housekeeping genes. Analysis revealed a significant main effect of treatment. This effect was driven by a significant difference within the HOM subjects that showed greater $2^{-\Delta C_{T}}$ values for the untreated (clean) animals compared to those treated with heroin. The utility of the comparative $C_{\mathrm{T}}$ method is predicated on the assumption that housekeeping gene expression is stable across treatment groups; this is essential as the housekeeping gene represents an internal control to which all target genes are compared. In this experiment a small but significant difference emerged within a single genotype (HOM) and between two of the three treatment groups (clean and heroin). This result may indicate a fundamental difference in the expression of the housekeeping between genotypes or treatment groups. However, if the genotypes were interacting with gene expression then it would be expected that all of the HOM animals, regardless of treatment, would show consistently altered gene expression. Similarly, if treatment were interacting with gene expression it would follow that all heroin treated groups would show this differential expression. Neither of these scenarios were supported by the current set of findings. Considering the total data presented in the Results section, a consistent pattern between all of the 
BDNF targets and both housekeeping genes was demonstrated. Therefore, this suggests that overall the relative expression of the two housekeeping genes is stable and that the effect seen here is likely the result of natural variation between discrete groups of animals.

When the five BDNF targets were compared to both Cyclophilin A and HPRT a differential pattern emerged between clean and MDMA treated subjects; a significant main effect of treatment was demonstrated for total BDNF, BDNF transcript III and BDNF transcript IV. That is, converted $2^{-\Delta C_{\mathrm{T}}}$ values were significantly greater in the MDMA treated groups compared to those that were untreated (clean) indicating increased BDNF target gene expression. Conversely, no differences emerged between clean and MDMA treated subjects when BDNF transcript VI and BDNF transcript IXa were evaluated. Despite an MDMA driven increase for total BDNF, BDNF transcript III and BDNF transcript IV and contrary to the hypothesis given above no genotype differences between clean and MDMA treated subjects emerged. This lack of effect was not expected considering the robust differences in MDMA self-administration behaviour exhibited by these animals. Considering the data carefully it is possible that a genotype effect may exist but is being obscured in the current experiment due to small samples sizes and a degree of variability.

Beyond the initial analysis the data were evaluated for the presence of outliers via various methods (e.g. outlier labelling rule); however, while the subsequent reanalysis trended closer to a significant effect it reduced the already small samples sizes and still failed to reveal a significant interaction or main effect of genotype for any of the BDNF targets. For this reason the analysis described in this chapter subsumes the entire data set and the raw data values are given in the Appendix for further inspection. In addition to evaluating MDMA induced differences in BDNF target gene expression analysis comparing the clean and heroin treated groups revealed no differences whatsoever for any of the five BDNF targets.

Taken together these results suggest that MDMA exposure facilitates 
gene expression for total BDNF and it does so equally for the three genotypes. This effect mirrors previously described research where the administration of duloxetine led to an increase in BDNF expression in both HOM and WT animals (Calabrese et al., 2010). Both duloxetine and MDMA target the SERT and NET and considering HOM animals have no SERT on which the drugs to act this suggests an important role of the NET in the MDMA induced changes in BDNF expression although this was not found in the striatum. The increased BDNF transcript IV expression after MDMA exposure is consistent with previous research demonstrating that cocaine facilitated increased expression of this particular BDNF isoform (Liu et al., 2006). These results are a direct contrast to the lack of effect in any of the BDNF targets for subjects treated with heroin. Previous research investigating the relationship between BDNF expression and opioid addiction has focused on changes associated with drug withdrawal and craving. For example, one group has demonstrated that total BDNF expression in the nucleus accumbens did not fluctuate after forced drug abstinence from heroin (Theberge et al., 2011, 2012); however, other research has demonstrated that total BDNF expression in the medial prefrontal cortex is increased after forced abstinence from heroin (Kuntz-Melcavage et al., 2009). It is important to note that the current data came from brain tissue that had been extracted and snap frozen without the animals experiencing drug withdrawal. That is, tissue extraction occurred after a single session of stable FR5 responding that followed the last progressive ratio dose. Therefore, it is possible that our results differ from others due to BDNF changes at varying time points during drug taking (immediately after progressive ratio versus after forced abstinence). Additionally, it should be stated that these experiments investigated BDNF expression changes in a variety of brain regions which could be differentially influenced after drug exposure. For these reasons it would be premature to conclude that heroin does not influence BDNF expression. Rather changes are likely contingent upon a variety of factors and these may not have been elucidated fully in the cur- 
rent set of experiments. Nonetheless, the current results show that there are inherent differences between the changes in striatal BDNF expression in animals that have self-administered MDMA compared to heroin.

Therefore, given the robust differences in MDMA self-administration between the genotypes the current qRT-PCR data indicate that changes in BDNF expression are not explicitly related to the development of drug addiction (as measured by drug self-administration). However, there are two important caveats that must be acknowledged. First, the research described above investigated changes in BDNF expression after drug withdrawal (Sadri-Vakili et al., 2010; H. D. Schmidt et al., 2012) whereas the current experiment did not. Therefore, it is unclear as to the exact role that BDNF plays in MDMA self-administration. Second, the current experiment only investigated changes in BDNF expression in animals that had successfully acquired MDMA self-administration (see Figure 2.2). It is important to remember that only about half of WT animals meet the acquisition criterion for MDMA self-administration. Therefore, while differences exist between the HOM and WT animals ("acquirers") across various schedules of responding the differences between the groups are not as large as those found for the acquisition of MDMA self-administration. This may explain why the current experiment demonstrated a robust effect for MDMA treated animals without revealing a difference between the genotypes. 
CHAPTER 4. QUANTITATIVE REVERSE TRANSCRIPTION 


\section{Chapter 5}

\section{General Discussion}

\subsection{Rationale}

The preceding series of behavioural and molecular experiments aimed to elucidate how a potential genetic risk factor, a reduction in SERT function, may influence the development of drug addiction. With only a small percentage of individuals who use drugs of abuse making the "transition to addiction" it behoves researchers to investigate the role that individual differences likely play in the development of drug addiction. Additionally, in order to develop and provide efficacious treatments for the clinic it is essential to understand the complex interplay between individual genetic differences and the types of drugs being consumed.

\subsection{Brief Summary}

Utilising the serotonin transporter knockout rat model HOM, HET and WT animals completed MDMA or heroin drug self-administration experiments. Interestingly, a robust genotype effect emerged for animals selfadministering MDMA; facilitation of MDMA self-administration was inversely related to reduced SERT function. HOM animals, without excep- 
tion, reached acquisition criterion significantly faster than the HET animals; HET animals showed higher acquisition rates compared to the WT animals. In contrast, there were no differences between the genotypes when animals self-administered heroin.

MDMA induced locomotor activity was reduced in HOM and HET animals compared to WT animals. However, heroin treatment did not produce differences in locomotion between the genotypes.

MDMA induced conditioned taste aversion revealed only a main effect of dose with robust conditioned taste aversion for both drug doses. However, heroin treatment failed to produce a conditioned taste aversion effect in any of the groups regardless of dose.

Lastly, when striatal brain tissue was analysed via qRT-PCR levels in several BDNF targets were significantly increased in animals that had selfadministered MDMA; this effect was true across genotypes. Comparatively, animals that had self-administered heroin did not show a difference in BDNF expression compared to untreated control animals.

\subsection{Experimental Significance}

The information gleaned from this series of behavioural and molecular experiments should be used to inform overall understanding of drug addiction in humans. Utilising the serotonin transporter knockout rat model was a very useful tool for systematically investigating how a reduction in the SERT contributes to the development of drug addiction. A major advantage of this tool is the fact that it effectively models a genetic conformation that happens naturally in humans; some individuals are born with reduced SERT function (s/s or 1/s) while others have what would be considered standard ( $1 / 1$, not reduced). To our knowledge there are no humans who completely lack the SERT like the HOM rat genotype. However, information gleaned from these animals can provide a valuable theoretical perspective compared to the other genotypes. Reduction in the 
SERT is seen in both humans (s/s) and the HET animals $50 \%$ compared to those with standard SERT function (1/1 in humans and WT animals). This change in the serotonergic system is present from conception in both humans and the serotonin transporter knockout rat or at least from the time the serotonergic system and the SERT start to develop. Consequently, it is reasonable to expect that compensatory mechanisms have occurred in response to this change.

By definition reduced SERT function does not allow for complete clearance of 5-HT from the synaptic space leaving it to continue acting on adjacent cells. Continually elevated levels of synaptic 5-HT are likely to lead to down regulation of 5-HT receptors which, in turn, would influence the entire serotonergic system (Homberg et al., 2008). At this point we do not fully understand the range, influence and complexity of these adaptations. Despite the fact that these changes complicate the understanding of the entire system they do exist in both humans and the SERT KO rat. Therefore, the results gleaned from these experiments can be carefully interpreted and inform understanding of human drug addiction. For example, the drug self-administration experiments demonstrated that animals with reduced SERT function are significantly more sensitive to the effects of MDMA compared to those with intact SERT function. This result suggests that depending on serotonergic function in humans some individuals may prove to be more sensitive to the effects of MDMA compared to others. Comparatively, regardless of genotype heroin self-administration behaviour was largely the same suggesting that a reduction in the SERT does not influence addiction to this particular opioid. However, as heroin has been well studied for many years it is known to be a powerful reinforcer and to have high abuse liability (indeed much higher than the abuse liability of MDMA). These results together suggest that a reduction in the SERT may confer increased susceptibility for developing drug addiction to certain types of drugs but perhaps not to others. That is, regardless of SERT function animals were equally as likely to administer heroin 
and become "addicted" whereas MDMA self-administration was significantly facilitated for animals with reduced SERT above levels for those with intact SERT function. However, it must be noted that the qRT-PCR analysis of the striatal brain tissue revealed that MDMA significantly increased BDNF expression in total BDNF and select transcripts but it did so equally across the three genotypes. This may be due to a variety of factors including small samples sizes, inability to detect small shifts in gene expression or that BDNF gene expression is more readily influenced by reduced SERT function in other portions of the brain (e.g. nucleus accumbens, frontal cortex, or prefrontal cortex). Conversely when the effect of heroin self-administration on BDNF expression was evaluated via qRTPCR the data were consistent with our behavioural findings; that is, no differences emerged when heroin treated subjects were compared to untreated controls. Taken together the current qRT-PCR experiments indicate that BDNF may play a role in drug addiction; however, at this time we cannot conclude that this relationship is direct and explicit.

It should also be noted that there is a great deal of literature supporting the idea that a complex relationship exists between the serotonergic system and disparate types of drugs. As described previously psychostimulants (e.g. cocaine, amphetamine) and opioids (e.g. morphine, heroin) ultimately act on a common neurological substrate (the mesolimbic dopamine pathway) which can lead to cognitive impairments in the prefrontal cortex. Interestingly, studies have shown that self-administration of cocaine or morphine does not predict self-administration of the other drug; these results reinforce the idea that psychostimulants and opioids operate by differing mechanisms (Lenoir, Guillem, Koob, \& Ahmed, 2012). More specifically, research has demonstrated that impulsivity, which is related to changes in the serotonergic system (Kirby et al., 2011) and is implicated in drug addiction, may provide insight into important differences between behavioural changes from the use of psychostimulants and opioids. For example, studies in human drug addicts revealed that compulsive am- 
phetamine use resulted in robust increases in measures of impulsivity (e.g. impairments in memory, decision making) whereas heroin use did not (Ersche, Clark, London, Robbins, \& Sahakian, 2006; Ersche et al., 2005). Similarly, animal studies demonstrated that cocaine withdrawal was accompanied by increased impulsivity (i.e. inhibitory control) whereas heroin withdrawal did not lead to changes in impulsivity (McNamara, Dalley, Robbins, Everitt, \& Belin, 2010; Winstanley et al., 2009).

Additionally, there is a body of research to support the differential role of specific serotonin receptors in the development of psychostimulant and opioid addiction (see Table 5.1). For example, one study investigated the role of $5-\mathrm{HT}_{3}$ receptors when amphetamine or morphine was selfadministered. They found that using specific $5-\mathrm{HT}_{3}$ antagonists, ICS 205930 and MDL 72222, effectively eliminated morphine induced conditioned place preference whereas they had no effect on amphetamine induced conditioned placed preference (Carboni, Acquas, Leone, \& Dichiara, 1989). Another study demonstrated that heroin induced DA release in the striatum was effectively inhibited when 5- $\mathrm{HT}_{4}$ antagonists, GR 125487 and SB 204070, were administered. Interestingly, striatal dopamine release induced by both amphetamine and cocaine were unaffected by administration of these $5-\mathrm{HT}_{4}$ antagonists (Porras, Di Matteo, De Deurwaerdere, Esposito, \& Spampinato, 2002). This same group also elegantly demonstrated that antagonism of $5-\mathrm{HT}_{2 \mathrm{~A}}$ and $5-\mathrm{HT}_{2 \mathrm{~B} / 2 \mathrm{C}}$ receptors led to drug specific changes in DA release in the nucleus accumbens and striatum. Specifically, the administration of $5-\mathrm{HT}_{2 \mathrm{~A}}$ antagonist, SR $46349 \mathrm{~B}$, inhibited amphetamine induced DA release whereas it had no effect on DA release produced by morphine. However, the $5-\mathrm{HT}_{2 \mathrm{~B} / 2 \mathrm{C}}$ antagonist, SB 206553, inhibited morphine induced DA release but failed to alter amphetamine induced DA release (Porras, Di Matteo, Fracasso, et al., 2002). These studies provide compelling evidence that disparate drugs of abuse, psychostimulants and opioids, have differential relationships with the serotonergic system. The results from the present thesis further emphasise the differ- 


\section{5-HT Receptors in Psychostimulant and Opioid Addiction}

\begin{tabular}{|l|l|}
\hline \hline Target Receptor: $5-\mathrm{HT}_{1 \mathrm{~A}}$ & Reference: Mosner et al. 1997 \\
\hline 5- $\mathrm{HT}_{1 \mathrm{~A}}$ receptor agonism increased morphine but not cocaine intake
\end{tabular}

\begin{tabular}{|c|c|}
\hline Target Receptor: $5-\mathrm{HT}_{2 \mathrm{~A} / 2 \mathrm{C}}$ & \\
\hline \multicolumn{2}{|c|}{$\begin{array}{l}5-\mathrm{HT}_{2 \mathrm{~A} / 2 \mathrm{C}} \text { receptor agonism prevented an increase in morphine but not } \\
\text { cocaine NAc DA release }\end{array}$} \\
\hline Target Receptor: $5-\mathrm{HT}_{2 \mathrm{~A}}, 5-\mathrm{HT}_{2 \mathrm{C} / 2 \mathrm{~B}}$ & Reference: Porras et al. 2002(a) \\
\hline \multicolumn{2}{|c|}{$\begin{array}{l}5-\mathrm{HT}_{2 \mathrm{~A}} \text { receptor antagonism reduced amphetamine but not morphine } \\
\mathrm{NAc} \text { DA release; } 5-\mathrm{HT}_{2 \mathrm{C} / 2 \mathrm{~B}} \text { antagonsim increased morphine but not } \\
\text { amphetamine NAc DA release }\end{array}$} \\
\hline
\end{tabular}

\begin{tabular}{l|l|}
\hline \hline Target Receptor: $5-\mathrm{HT}_{3}$ & Reference: Carboni et al. 1989 \\
\hline 5- $\mathrm{HT}_{3}$ receptor antagonism reduced morphine but not amphetamine \\
induced CPP
\end{tabular}

\begin{tabular}{|l|l|}
\hline \hline Target Receptor: $5-\mathrm{HT}_{4}$ & Reference: Porras et al. 2002 \\
\hline 5- $\mathrm{HT}_{4}$ receptor antagonism reduced morphine but not amphetamine \\
or cocaine strital DA release
\end{tabular}

\begin{tabular}{|l|l|}
\hline \hline Target Receptor: $5-\mathrm{HT}_{2 \mathrm{C}}$ & Reference: Schmidt et al. 1992 \\
\hline 5- $\mathrm{HT}_{2 \mathrm{C}}$ receptor antagonism attenuated MDMA induced increases in \\
extracellular striatal DA
\end{tabular}

\begin{tabular}{|l|l|}
\hline \hline Target Receptor: $5-\mathrm{HT}_{2 \mathrm{C}}$ & Reference: Gudelsky et al. 1994 \\
\hline 5- $\mathrm{HT}_{2 \mathrm{C}}$ receptor agonism facilitated MDMA induced increases in ex- \\
tracellular striatal DA
\end{tabular}

Table 5.1: Specific 5-HT receptor interactions with psychostimulants and opioids. 
ential role 5-HT plays in the reinforcing properties of drugs of abuse. Although the previous studies focused predominantly on morphine versus amphetamine, our experiments show that differences also exist between heroin and MDMA. While it is well known that a genetic reduction in the SERT leads to downregulation of 5-HT receptors most of this evidence has come from investigation of the $5-\mathrm{HT}_{1 \mathrm{~A}}$ receptor. Given that MDMA is significantly more reinforcing for HOM and HET animals (compared to the WT), the current results point to the crucial involvement of 5-HT and possibly specific 5-HT receptors. As discussed in previous chapters, heroin only indirectly stimulates release of DA in the mesolimbic pathway via inhibition of GABA interneurons. The data from Chapter 2 showed that there were no differences between the genotypes in relation to heroin selfadministration behaviour; this suggests that the 5-HT receptors involved in MDMA self-administration do not play an important role when heroin is self-administered. The crucial question is: Of the various serotonin receptors, which ones are predominantly involved in the reinforcing properties of MDMA? At present we cannot answer this question as the role of many of these receptors has yet to be determined. Additionally, specific 5 -HT receptor downregulation in the SERT KO model has not been fully elucidated.

Furthermore, studies to date investigating heroin addiction in humans fail to provide a consistent account of how reduced SERT influences the development of addictive behaviours. Some studies indicate that reduced SERT function does indeed represent a risk factor in the development of heroin addiction (Gerra et al., 2004; Saiz et al., 2008; Tan et al., 1999; Yang et al., 2012); however other studies have failed to find such a relationship (Kotler et al., 1999; T. Li et al., 2002; Saiz et al., 2009). The current data does not provide support for this relationship; these mixed results may reflect a variety of factors. As described in Chapter 2 there are several potential confounding variables that may explain the disparate results in the human data. Additionally, the parameters of the current heroin self- 
administration experiment ( 2 hour sessions) may have limited the scope of responding for the drug. It is possible that with longer access to the drug a genotype difference may have emerged; an investigation into responding for heroin with longer access to the drug may provide a useful comparison to the current data and elucidate a genotype effect if indeed one exists.

The BDNF gene expression analysis suggests that MDMA exposure facilitates gene expression for total BDNF and, in the current experiment, it does so equally for the three genotypes. As described in Chapter 4 this effect mirrors previously described research where the administration of duloxetine led to an increase in hippocampal and prefrontal cortex BDNF expression in both HOM and WT animals (Calabrese et al., 2010). Both duloxetine and MDMA target the SERT and NET but HOM and HET animals either completely lack or have reduced SERT therefore this may suggest an important role of the NET in the MDMA induced changes in BDNF expression. These results are a direct contrast to the lack of effect in any of the BDNF targets for subjects treated with heroin. Previous research investigating the relationship between BDNF expression and opioid addiction is less clear, focusing on changes associated with drug withdrawal and craving. At this time it would be premature to conclude that heroin does not change BDNF expression; it is possible that changes may be concentrated in other areas of the brain or that heroin exposure more readily influences BDNF expression after drug abstinence. Only additional studies will effectively elucidate the relationship between heroin and BDNF gene expression.

\subsection{Limitations}

While the research described in this thesis forms a coherent set of experiments that can inform understanding of human drug addiction there are some important limitations that must be acknowledged. Each behavioural and molecular paradigm is discussed in turn followed by a discussion of 
the challenges of translating animal research to inform outcomes for humans.

\subsubsection{Novelty Suppressed Feeding}

It is widely accepted that the mechanisms that underlie drug addiction are highly complex. Additionally, the high incidence of comorbidity between drug addiction and anxiety disorders complicates studying and understanding this phenomenon in the laboratory. Therefore, in an effort to tease apart the relationship between anxiety and self-administration behaviour subjects first completed novelty suppressed feeding to evaluate differences in anxiety between groups. This paradigm had been used previously by colleagues who found robust and reliable results; namely, that reduced SERT function corresponded to higher levels of anxiety (Olivier et al., 2008). For this reason we selected it as a measure of anxiety before subjects went on to drug self-administration. Unexpectedly, we failed to find a reliable difference in anxiety between the genotypes. As consistent methodology was employed this result may reflect a degree of natural variation between different groups of animals. Alternatively, it may suggest that small differences in experimental variables may have affected the measured behaviour in these particular experiments. Practically, it should be noted that a large number of subjects underwent novelty suppressed feeding, self-administration surgery and testing. Therefore, smaller groups were staggered across the process instead of taking the entire lot through each individual step before starting the next one. In short, when the results from the novelty suppressed feeding paradigm were completed a large number of subjects had already undergone catheter implantation and started self-administration testing. Therefore, while it would have interesting to attempt another paradigm to measure anxiety it would have been highly impractical at this stage of the experimental procedures. For these reasons a single test of anxiety was used in the current 
set of experiments.

\subsubsection{Drug Self-Administration}

As discussed previously, drug self-administration is a powerful tool for evaluating drug addiction in humans and animals and is used widely in laboratories around the world. However, it carries its own limitations. Drug addiction, as described in the General Introduction, is currently defined as a set of behaviours described in the DSM-5: namely, difficulty limiting use of the drug, increasing motivation to acquire the drug and lastly continued use despite negative consequences. Now the current drug self-administration experiments demonstrated that animals were increasingly motivated to acquire the drug (MDMA) as evidenced by increasing breakpoints on a progressive ratio schedule. However, these experiments did not model continued drug use despite negative consequences. At no point were animals punished for their drug taking behaviour (as with a foot shock or other aversive stimulus) and for this reason it should be acknowledged that these experiments did not model all aspects of drug addiction as set forth in the DSM-5. Some of these indices could be evaluated using extended access sessions (6 hours) or exposure to schedules of reinforcement where drug associated cues or stimuli are presented to the animal (Everitt et al., 2008).

Additionally, drug self-administration experiments rarely provide an animal with an alternative choice to the drug. This has prompted the investigation of choice within a drug self-administration experiment. Interestingly, results have shown that when rats are given a choice between cocaine and an alternative reward they seldom choose cocaine even when the dose is increased. However, these studies did discover a small group of rats that would forgo the alternative reward and self-administer cocaine; these particular animals may prove to be a more accurate model of the small group of individuals who make the transition to addiction 
(Ahmed, 2012). Considering that drug self-administration in the laboratory is meant to mirror drug self-administration in humans it should reflect the fact that humans often face a variety of choices when it comes to drug taking. Therefore, this aspect of drug self-administration cannot be ignored and should be incorporated into laboratory paradigms of drug self-administration.

Also, it should be noted that the current MDMA and heroin self-administration experiments in the SERT KO rat model are some of the first of their kind. While the results reported in this thesis are compelling they are far from complete. As such it will be imperative to incorporate a full set of dose response curves into future drug self-administration experiments. This will help to elucidate the specific effects of dose and how they relate to the reinforcing properties of these drugs of abuse.

Lastly, there is increasing discussion regarding the use of both male and female subjects to study a variety of psychiatric illness, including drug addiction, in the laboratory. Before the current set of experiments was undertaken a pilot study was conducted by our group to evaluate MDMA self-adminstration in HOM and WT subjects (Oakly et al., 2014). Initially, we wanted to compare our results with those from another group that has been intensively studying MDMA self-administration in male SpragueDawley rats (Schenk, 2009, 2011). To make the direct comparison we used male subjects and found that the HOM animals were significantly more sensitive to the effects of MDMA compared to the WT. Moving forward, we began the set of experiments outlined in this thesis. As a small laboratory we maintain our own breeding colony and at the time were struggling to produced a sufficient number of male subjects for each genotype. For this reason an MDMA self-administration experiment with HOM and HET subjects (both male and female) was undertaken. Surgical procedures commenced and when subjects had recovered they began MDMA self-administration. On their first day in the self-administration chamber three females rapidly administered $30+$ infusions within the first twenty 
minutes of the session. Considering these subjects had no prior experience with MDMA such a large amount administered so quickly was lethal. This effect was not seen in any of the males in this experiment. It was decided that it would be unethical to subject the remaining female subjects to a potential drug overdose and therefore the experiment was terminated. From there animals were bred until there was a sufficient number of male subjects for each genotype.

\subsubsection{Locomotor Activity}

Drug influenced locomotion is an important adjunct to drug self-administration experiments. Locomotor activity is a relatively straightforward way to quantify levels of activity after drug exposure. For example, heroin is classified as a narcotic and produces drowsiness after consumption. High levels of sedation could run counter to and prevent drug self-administration behaviour (lever pressing) and therefore it was important to evaluate levels of locomotor activity under a variety of heroin doses. In this way the heroin locomotor activity experiments demonstrated that across genotypes animals demonstrated largely stable levels of locomotor activity. Therefore, while heroin can produce sedation this was not the reason for reduced drug self-administration behaviour compared to that seen with MDMA.

MDMA induced locomotion highlighted the fact that not all drugs of abuse produce the same pattern of locomotor activity. That is, drugs of abuse lead to a common downstream effect, dopamine release and this dopaminergic activity is thought to underlie the reinforcing effects of drugs of abuse as well as locomotor activity. Interestingly, according to the current data MDMA induced locomotor activity is not driven by dopaminergic activity but is much more contingent upon 5-HT release. For this reason utilising locomotor activity as a measure of the reinforcing properties of drugs like MDMA may not be accurate. 


\subsubsection{Conditioned Taste Aversion}

Traditionally, conditioned taste aversion has been used to evaluate the negative consequences of drugs of abuse. This idea was initially posited when conditioned taste aversion was demonstrated in animals; specifically, drugs that were readily self-administered also caused conditioned taste aversion and this suggested that drugs of abuse may have both positive and negative properties (Hunt \& Amit, 1987). In the current experiments conditioned taste aversion developed after sucrose was paired with MDMA and while it would fit with some historical data suggesting that the negative properties of MDMA were driving the effect there is additional research to suggest an alternative explanation. There is growing support for the idea that conditioned taste aversion does not reflect the negative properties of drugs of abuse but rather the positive, reinforcing properties (Grigson, 1997). Proponents of this revised theory suggest that the decreased consumption of the palatable solution is due to the fact that the powerful reinforcing effects of the drug outweigh, effectively devalue, the desirability of this natural reinforcer. It is this devaluation of the palatable solution that drives decreased consumption; in contrast to the highly desirable drug effect the gustatory reward is greatly diminished.

Other researchers have argued that reduced consumption of the palatable solution merely reflects an uncertainty where an animal associates the drug effect, a change the normal physiological state, with the palatable solution. Since avoiding ingestion of potential toxins lies at the core of conditioned taste aversion it follows that a fluctuation in the normal physiological state may be interpreted as a toxin and therefore becomes associated with the palatable solution (Lin, Arthurs, \& Reilly, 2014).

If these explanations are accurate the current MDMA conditioned taste aversion results suggest that the positive, reinforcing properties of MDMA devalue the palatable solution and this is evidenced by reduced sucrose consumption. Alternatively, the MDMA drug effects may have been interpreted as a potential toxin, whether they were or not, and conditioned 
taste aversion developed in an effort to avoid this potentially harmful substance.

Considering the current heroin conditioned taste aversion data the aforementioned explanations fail to account for the lack of affect when heroin was paired with the sucrose solution. If the devaluation hypothesis was correct then the powerful reinforcing properties of heroin would be expected to unequivocally inhibit sucrose consumption. Considering there was no effect, not even a trend, for either drug dose this argues against the devaluation hypothesis. Additionally, the idea that changes in the physiological state may be enough to elicit a conditioned taste aversion does not provide a compelling explanation for the current data. If this idea were correct it would be expected that the heroin induced physiological change, that may be considerably stronger than that of MDMA, would have caused a marked decrease in sucrose consumption. Considering the current data and the ongoing debate that surrounds conditioned taste aversion it is obvious that additional research is needed to fully understand the mechanism driving this behavioural phenomenon.

\subsubsection{Quantitative Reverse Transcription Polymerase Chain Reaction}

Quantitative reverse transcription polymerase chain reaction (qRT-PCR) has become the "go-to" technique for evaluating gene expression and has significant advantages. Most notably qRT-PCR is a sensitive technique and can be used to quantify relatively small amounts of RNA in a sample. Additionally, qRT-PCR has high throughput where multiple samples can be processed simultaneously as well as the evaluation of multiple genes in a single sample. With this said qRT-PCR also has some limitations that must be addressed. One potential pitfall lies in the amplification process; that is, each cycle produces a doubling of amplification products. If a portion of these products are erroneous (e.g. genomic DNA) this will lead to mul- 
tifold amplification of errors which will add large variability to a sample and make interpretation difficult (Zimmermann \& Mannhalter, 1996).

More specifically, the reverse transcription portion of a qRT-PCR experiment has the potential to increase variability. This is due to the sensitivity of the reverse transcriptase enzyme; it can be influenced by even small amounts of salt, alcohol and/or phenol leading to varying efficiency in the reverse transcription reaction. It is for this reason that precise RNA isolation is absolutely essential; clean RNA will ensure higher efficiency when reverse transcribed into the cDNA template (Freeman, Walker, \& Vrana, 1999).

Lastly, the qRT-PCR experiments described in this thesis focus on brain tissue taken from the striatum. The entire structure was excised and processed due to a small degree of variability/imprecision in the slicing technique. As discussed in the General Introduction there are important functional differences within the various portions of the striatum; for example, habitual drug taking is accompanied by a ventral to dorsal and then a dorsomedial to dorsolateral shift. Therefore, in subsequent experiments it would be prudent to implement a different slicing technique where individual subsections could be isolated.

\subsubsection{Translating Animal Outcomes to Humans}

An inherent limitation to the whole of this thesis is the fact that all experiments were performed in animals with a goal to inform human drug addiction. It must be acknowledged that animal models, at their core, are approximations of pathophysiology that is seen in humans and for this reason interpreting these data and what they may mean for human drug addiction must be done with caution (Ahmed, 2010; McGonigle \& Ruggeri, 2014).

With that said animal models can be powerful tools for the systematic study of behavioural and neurochemical changes in the laboratory. It is 
for this reason that we chose to utilise the SERT KO rat model to ask specific questions regarding genetic risk and drug addiction. Considering the complex interplay of changes in behaviour and the brain that accompany drug addiction it would have been impossible to conduct these experiments in vitro or using non-biological methods like computer modelling. While non animal alternatives are being developed for many types of laboratory research none have been developed to date that would entirely replace the use of an animal model for the suite of experiments described in this thesis.

\subsection{Future Directions}

This thesis set out to ask and answer several questions regarding genetic risk and drug addiction. While the results as a whole will serve to inform the body of research regarding drug addiction they also beg several additional questions. Therefore, the following sections outline several ideas for future research questions and how to go about answering them.

\subsubsection{Microdialysis and In Vivo Voltammetry}

The SERT KO rat model is a relatively new tool for investigating the influence of genetic risk on the development of drug addiction. While there is ongoing research to understand the complete behavioural and neurochemical profile of this particular model there are still questions to be answered. Recent research has evaluated DA and 5-HT release in target brain structures after administration of cocaine and found that DA release was largely similar in HOM and WT animals (Verheij et al., 2014). Moving forward it would prove useful to directly measure extracellular concentrations of both 5-HT and DA after MDMA self-administration using microdialysis and high performance liquid chromatography (HPLC) (Benveniste \& Httemeier, 1990; Ungerstedt, 1991). Initially, we had hy- 
pothesised that animals with reduced SERT function may have a sensitised dopaminergic system however we were unable to definitively ascertain if this were true. Measuring DA and 5-HT release may provide insight into the complex relationship of these two neurotransmitter systems and how they interact when a drug like MDMA is administered. It should be noted that microdialysis measures changes in analytes (chemical constituents) over several minutes; therefore, this technique may not provide the resolution necessary to detect small changes that likely occur after exposure to a drug. To better investigate small changes in neurotransmitter levels a technique with more precise sampling may be useful. One such technique, in vivo voltammetry, provides a measure of neurotransmitter concentration by quantifying molecule oxidation near an inserted electrode (Greco \& Garris, 2003; Justice, Michael, \& Neill, 1985; Salvatore, Hoffman, Burmeister, \& Gerhardt, 2003). Measurements taken from in vivo voltammetry describe rapidly changing neurotransmitter levels and may provide insight into the complex, transient changes that occur when a drug of abuse is applied to the system.

\subsubsection{Ambiguous Cue Interpretation}

Considering the discussion of locomotor activity and conditioned taste aversion given above it is clear that further investigation into the positive and negative properties of drugs of abuse is needed. To this end a new behavioural paradigm, ambiguous cue interpretation, has been developed; this test provides an opportunity to investigate the positive and negative effects of drugs of abuse simultaneously. That is, rats are trained to press a lever when they hear a specific tone to receive a food reward; additionally, they are also trained to press a lever when they hear a different tone in order to avoid a foot shock. After training to a level of stable responding rats are presented with an intermediate tone; responding on one lever or the other would indicate that the rat perceives the ambiguous tone as a posi- 
tive or negative event (Enkel et al., 2010; Papciak, Popik, Fuchs, \& Rygula, 2013). It is suggested that this procedure could be utilised to evaluate the positive and negative effects of drugs of abuse; after drug treatment rats could then "self-report" by selecting the lever that most closely matches the drug effect they experience.

\subsubsection{Emulsion PCR}

As discussed above a potential limitation of qRT-PCR is the precision in quantifying amplification products. This is particularly problematic when there are very small amounts of the target gene in a selected sample; resulting $C_{\mathrm{T}}$ values will often approach the maximum number of cycles in the reaction. It is in these last few cycles where amplification of errors would be most exaggerated. To increase precision particularly when samples contain very few target sequences methods beyond qRT-PCR must be utilised. There is growing support for a technique called emulsion polymerase chain reaction (ePCR). Recently developed, ePCR allows for absolute quantification of amplification products; using PCR amplification on individual water-in-oil droplets allows for high precision in detecting whether a target sequence is present in a sample or not (Williams et al., 2006; Zhu et al., 2012). ePCR provides a useful and increasingly elegant alternative to $\mathrm{qRT}$-PCR for evaluating target gene expression.

\subsection{Final Conclusion}

The current body of work set out to address this question: Is the serotonin transporter a risk factor in the development of drug addiction? Considering the data gleaned from this series of behavioural and molecular experiments it is clear that to fully answer this question additional research must be undetaken. Limitations of the current research have been acknowledged and discussed above; additionally, ideas for future research have 
also been outlined. However, it is insufficient to conclude that the current work cannot be interpreted and would only be valid with additional experimental data sets. Therefore, a preliminary conclusion is provided for consideration and is based solely on the data presented in this thesis. The combined results point to a potentially compelling relationship between the serotonin transporter and specific drugs of abuse that target this protein. In the end, the scientific community is trying to understanding why some individuals become addicted to drugs of abuse whereas others do not. While it is readily ackowledged that this is no simple line of inquiry new data like what is presented in this thesis can provide a fundamental, essential foundation for the research that will ultimately answer this question. 


\section{Appendix A}

\section{Supplemental qRT-PCR Analysis}

Supplemental information for the qRT-PCR experiments described in Chapter 4 is given in this Appendix.

- Section 1: Housekeeping Genes

- Section 2: Total BDNF

- Section 3: BDNF transcript III

- Section 4: BDNF transcript IV

- Section 5: BDNF transcript VI

- Section 6: BDNF transcript IXa

\section{A.1 Section 1: Housekeeping Genes}

Relative expression of HPRT against Cyclophilin A is described in Chapter 4. $\Delta C_{\mathrm{T}}$ values were calculated for each sample $\left(\Delta C_{\mathrm{T}}=\left(C_{\mathrm{T}} \mathrm{HPRT}\right)-\left(C_{\mathrm{T}}\right.\right.$ Cyc A)) (see Figure A.1). Each $\Delta C_{T}$ value was converted into the negative binary $\log \left(2^{-\Delta C_{T}}\right)$ (see Figure A.2). Raw datum values are given in Tables A.1, A.2 and A.3. 


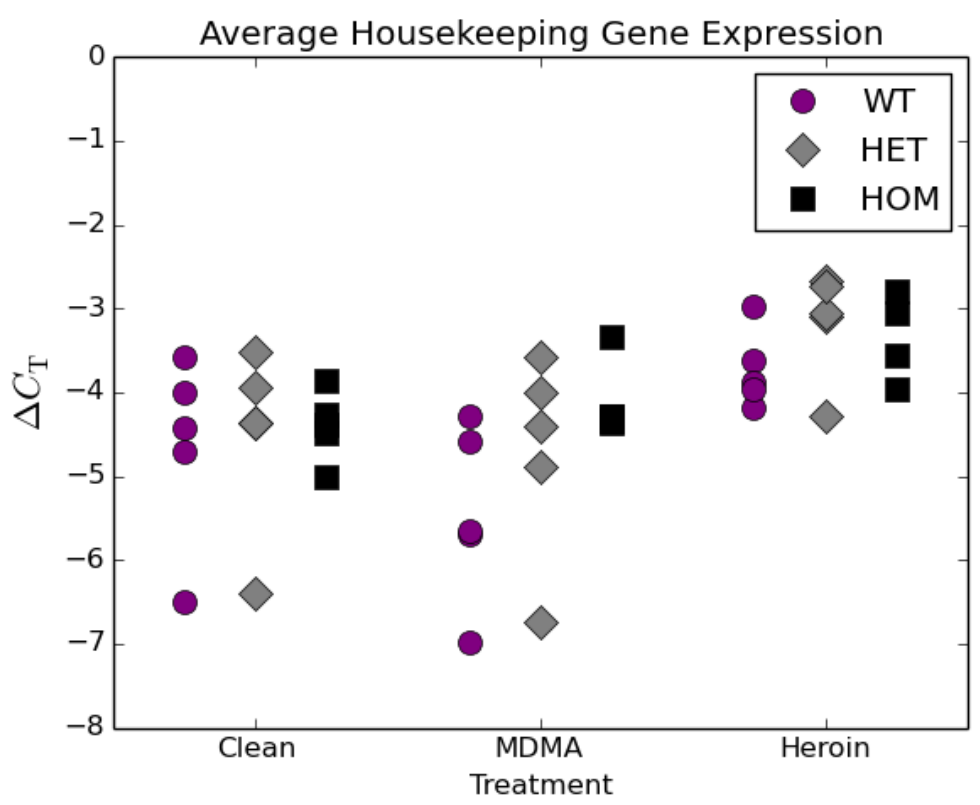

Figure A.1: $\Delta C_{\mathrm{T}}$ values for HPRT against Cyc A across treatment groups and genotypes.

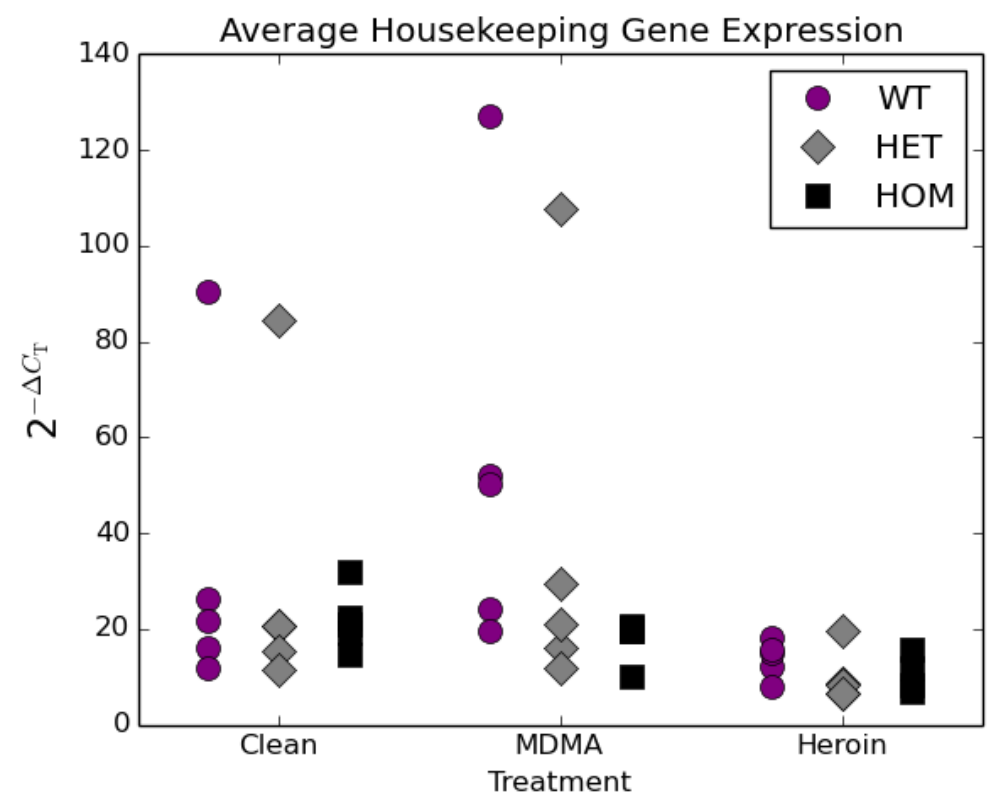

Figure A.2: $2^{-\Delta C_{\mathrm{T}}}$ values for HPRT against Cyc A across treatment groups and genotypes. 


\begin{tabular}{|c|c|c|c|c|}
\hline \multicolumn{5}{|c|}{ HOM } \\
\hline \multicolumn{5}{|l|}{ Clean } \\
\hline Animal & HPRT $C_{\mathrm{T}}$ & Сус А $C_{\mathrm{T}}$ & $\Delta C_{\mathrm{T}}$ & $2^{-\Delta C_{T}}$ \\
\hline 117 & 23.23 & 28.23 & -5 & 32 \\
\hline 119 & 23.07 & 27.46 & -4.39 & 20.97 \\
\hline 122 & 23.7 & 28.18 & -4.48 & 22.32 \\
\hline 123 & 22.72 & 26.98 & -4.26 & 19.16 \\
\hline 121 & 23.65 & 27.51 & -3.86 & 14.52 \\
\hline \multicolumn{5}{|l|}{ MDMA } \\
\hline Animal & HPRT $C_{\mathrm{T}}$ & Сус A $C_{\mathrm{T}}$ & $\Delta C_{\mathrm{T}}$ & $2^{-\Delta C_{T}}$ \\
\hline 210 & 22.43 & 25.77 & -3.34 & 10.13 \\
\hline 223REP & 22.43 & 26.78 & -4.36 & 20.46 \\
\hline 249REP & 23.14 & 27.43 & -4.29 & 19.56 \\
\hline 415 & 23.09 & 27.44 & -4.35 & 20.39 \\
\hline 423 & 21.66 & 25.94 & -4.28 & 19.43 \\
\hline \multicolumn{5}{|l|}{ Heroin } \\
\hline Animal & HPRT $C_{\mathrm{T}}$ & Сус A $C_{\mathrm{T}}$ & $\Delta C_{\mathrm{T}}$ & $2^{-\Delta C_{T}}$ \\
\hline 710 & 22.07 & 25.12 & -3.05 & 8.28 \\
\hline 719 & 22.74 & 26.3 & -3.56 & 11.79 \\
\hline 720 & 22.99 & 25.79 & -2.8 & 6.96 \\
\hline 742REP & 21.11 & 25.08 & -3.97 & 15.67 \\
\hline
\end{tabular}

Table A.1: Comparison of housekeeping gene expression: HOM subjects across treatment groups. 


\begin{tabular}{|c|c|c|c|c|}
\hline \multicolumn{5}{|c|}{ HET } \\
\hline \multicolumn{5}{|l|}{ Clean } \\
\hline Animal & ${ }_{\text {HPRT }} C_{T}$ & Сус $\mathbf{A} C_{\mathrm{T}}$ & $\Delta C_{\mathrm{T}}$ & $2^{-\Delta C_{T}}$ \\
\hline 110 & 24.34 & 30.74 & -6.4 & 84.45 \\
\hline 111 & 23.09 & 27.45 & -4.36 & 20.53 \\
\hline 112 & 21.97 & 26.33 & -4.36 & 20.55 \\
\hline 114 & 22.25 & 26.2 & -3.95 & 15.45 \\
\hline 116 & 22.76 & 26.28 & -3.52 & 11.47 \\
\hline \multicolumn{5}{|l|}{ MDMA } \\
\hline Animal & ${ }_{\text {HPRT }} C_{T}$ & Сус А $C_{\mathrm{T}}$ & $\Delta C_{\mathrm{T}}$ & $2^{-\Delta C_{\mathrm{T}}}$ \\
\hline 217 & 22.82 & 26.83 & -4.01 & 16.11 \\
\hline 231 & 21.78 & 25.36 & -3.58 & 11.96 \\
\hline 232 & 22.83 & 27.23 & -4.4 & 21.11 \\
\hline 246 & 21.62 & 26.5 & -4.88 & 29.45 \\
\hline 247 & 24.3 & 31.05 & -6.75 & 107.63 \\
\hline \multicolumn{5}{|l|}{ Heroin } \\
\hline Animal & ${ }_{\text {HPRT }} C_{T}$ & Сус А $C_{\mathrm{T}}$ & $\Delta C_{\mathrm{T}}$ & $2^{-\Delta C_{T}}$ \\
\hline 704 & 23.75 & 26.43 & -2.68 & 6.41 \\
\hline 715 & 22.69 & 25.78 & -3.09 & 8.51 \\
\hline 723 & 23.97 & 28.25 & -4.28 & 19.43 \\
\hline 732 & 21.92 & 24.97 & -3.05 & 8.28 \\
\hline 733 & 23.44 & 26.18 & -2.74 & 6.68 \\
\hline
\end{tabular}

Table A.2: Comparison of housekeeping gene expression: HET subjects across treatment groups. 


\begin{tabular}{|r|c|c|c|c|}
\hline \multicolumn{5}{|l|}{ WT } \\
\hline \multicolumn{1}{|l|}{ Clean } \\
\hline Animal & HPRT $\boldsymbol{C}_{\mathbf{T}}$ & Cyc A $\boldsymbol{C}_{\mathbf{T}}$ & $\Delta \boldsymbol{C}_{\mathbf{T}}$ & $\mathbf{2}^{-\Delta \boldsymbol{C}_{\mathrm{T}}}$ \\
\hline 102 & 22.33 & 28.83 & -6.5 & 90.51 \\
\hline 103 & 22.79 & 27.5 & -4.71 & 26.17 \\
\hline 104 & 21.59 & 25.59 & -4 & 16 \\
\hline 106 & 23.09 & 26.67 & -3.58 & 11.96 \\
\hline 108REP & 21.99 & 26.42 & -4.44 & 21.63 \\
\hline
\end{tabular}

\begin{tabular}{|r|c|c|c|c|}
\hline \hline \multicolumn{5}{|l|}{ MDMA } \\
\hline Animal & HPRT $\boldsymbol{C}_{\mathbf{T}}$ & Cyc A $\boldsymbol{C}_{\mathbf{T}}$ & $\Delta \boldsymbol{C}_{\mathrm{T}}$ & $\mathbf{2}^{-\Delta \boldsymbol{C}_{\mathrm{T}}}$ \\
\hline 203 & 23.04 & 30.03 & -6.99 & 127.12 \\
\hline 216 & 23.31 & 29.01 & -5.7 & 51.98 \\
\hline 240 & 23.44 & 29.09 & -5.65 & 50.21 \\
\hline 401 & 21.93 & 26.52 & -4.59 & 24.08 \\
\hline 417 & 23.12 & 27.41 & -4.29 & 19.56 \\
\hline \hline
\end{tabular}

\begin{tabular}{|r|c|c|c|c|}
\hline \multicolumn{5}{|l|}{ Heroin } \\
\hline Animal & HPRT $\boldsymbol{C}_{\mathbf{T}}$ & ${\text { Cyc A } \boldsymbol{C}_{\mathbf{T}}}$ & $\Delta \boldsymbol{C}_{\mathbf{T}}$ & $\mathbf{2}^{-\Delta \boldsymbol{C}_{\mathbf{T}}}$ \\
\hline 701 & 23.78 & 27.39 & -3.61 & 12.21 \\
\hline 711 & 22.29 & 25.26 & -2.97 & 7.84 \\
\hline 721 & 22.51 & 26.69 & -4.18 & 18.13 \\
\hline 730 & 22.43 & 26.32 & -3.89 & 14.83 \\
\hline 739 & 22.81 & 26.76 & -3.96 & 15.51 \\
\hline
\end{tabular}

Table A.3: Comparison of housekeeping gene expression: WT subjects across treatment groups. 


\section{A.2 Section 2: Total BDNF}

Relative expression of total BDNF (against Cyclophilin A and HPRT) was described in Chapter 4.

- $\Delta C_{\mathrm{T}}$ values were calculated for each sample against Cyclophilin $\mathrm{A}$, $\left(\Delta C_{\mathrm{T}}=\left(C_{\mathrm{T}}\right.\right.$ total BDNF) - $\left(C_{\mathrm{T}}\right.$ Cyc A $)$ ) (see Figure A.3), and HPRT $\left(\Delta C_{\mathrm{T}}=\left(C_{\mathrm{T}}\right.\right.$ total BDNF) $\left.-\left(C_{\mathrm{T}} \mathrm{HPRT}\right)\right)$ (see Figure A.4); raw datum values are given in Table A.4.

- Each $\Delta C_{\mathrm{T}}$ value was converted into the negative binary $\log \left(2^{-\Delta C_{\mathrm{T}}}\right)$ (see Figures A.5 and A.6); raw datum values are given in Table A.5.

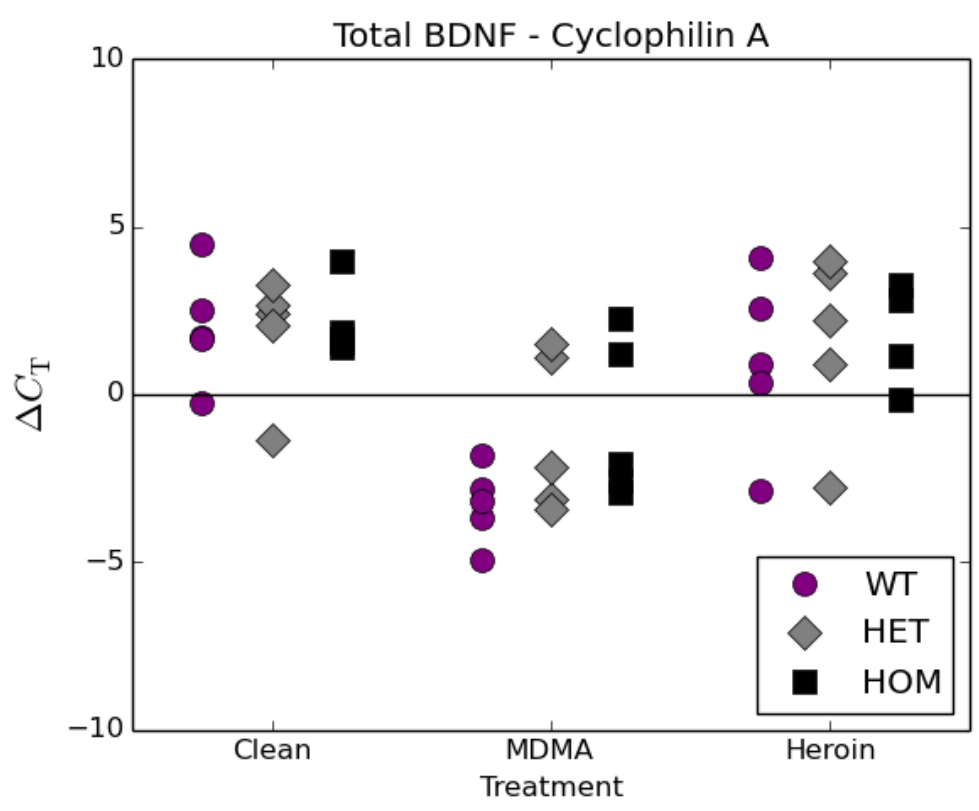

Figure A.3: $\Delta C_{\mathrm{T}}$ values for total BDNF against $\mathrm{Cyc} \mathrm{A}$. 


\begin{tabular}{|c|c|c|}
\hline \multicolumn{3}{|c|}{ Cyclophilin A } \\
\hline \hline Clean \\
\hline HOM & HET & WT \\
\hline 1.57 & 2.41 & 1.69 \\
\hline 1.84 & 2.67 & -0.24 \\
\hline 1.38 & 3.28 & 4.49 \\
\hline 3.99 & -1.36 & 1.66 \\
\hline 1.68 & 2.04 & 2.51 \\
\hline \hline MDMA \\
\hline HOM & HET & WT \\
\hline-2.59 & 1.1 & -4.93 \\
\hline-2.06 & 1.5 & -3.67 \\
\hline 1.23 & -3.15 & -2.83 \\
\hline-2.93 & -2.17 & -1.84 \\
\hline 2.24 & -3.43 & -3.18 \\
\hline \hline Heroin \\
\hline HOM & HET & WT \\
\hline 2.79 & -2.79 & -2.90 \\
\hline 3.29 & 3.63 & 4.07 \\
\hline-0.14 & 2.22 & 0.9 \\
\hline 1.15 & 4.00 & 0.34 \\
\hline- & 0.93 & 2.59 \\
\hline
\end{tabular}

(a)

\begin{tabular}{|c|c|c|}
\hline \multicolumn{3}{|c|}{ HPRT } \\
\hline \hline Clean \\
\hline HOM & HET & WT \\
\hline 5.43 & 6.36 & 6.39 \\
\hline 6.83 & 7.04 & 6.26 \\
\hline 5.65 & 6.8 & 8.08 \\
\hline 8.39 & 5.04 & 5.67 \\
\hline 6.16 & 6.4 & 6.94 \\
\hline \hline MDMA \\
\hline HOM & HET & WT \\
\hline 1.69 & 4.68 & 0.77 \\
\hline 2.30 & 5.51 & 1.99 \\
\hline 4.57 & 1.26 & 1.46 \\
\hline 1.42 & 4.59 & 5.15 \\
\hline 6.52 & 1.45 & 1.42 \\
\hline \hline Heroin \\
\hline HOM & HET & WT \\
\hline 6.35 & 1.49 & 1.06 \\
\hline 7.26 & 6.31 & 7.68 \\
\hline 2.91 & 5.32 & 3.87 \\
\hline 3.95 & 6.73 & 4.23 \\
\hline- & 3.98 & 6.77 \\
\hline
\end{tabular}

(b)

Table A.4: $\Delta C_{\mathrm{T}}$ values for total BDNF against Cyclophilin A (a) and HPRT (b). 


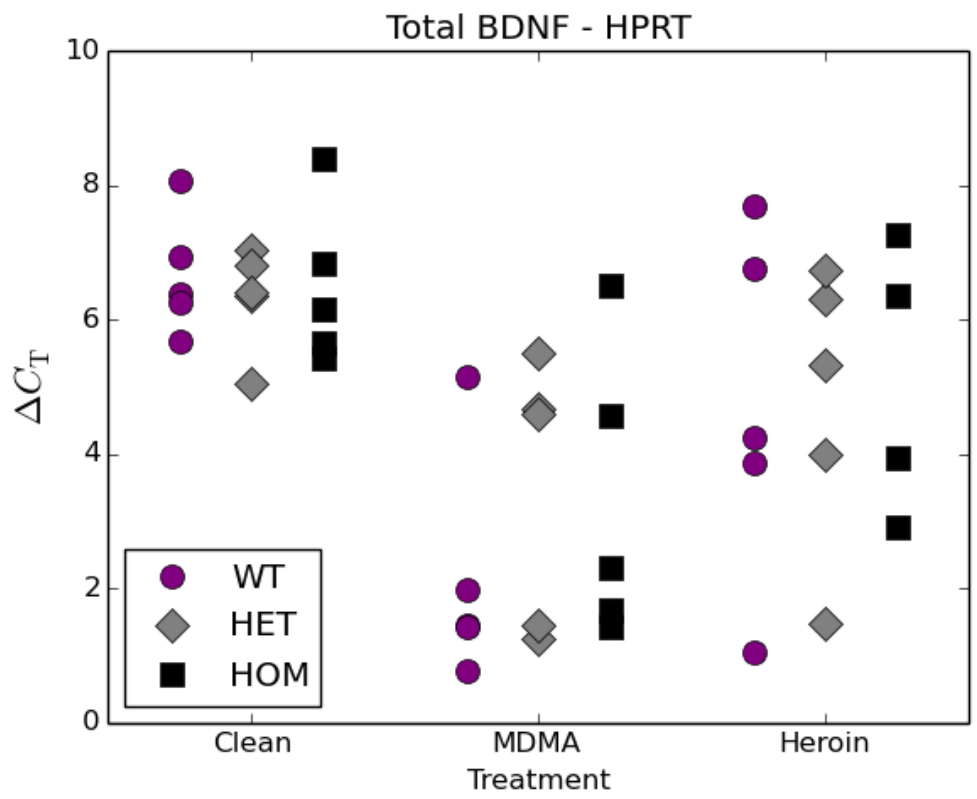

Figure A.4: $\Delta C_{\mathrm{T}}$ values for total BDNF against HPRT.

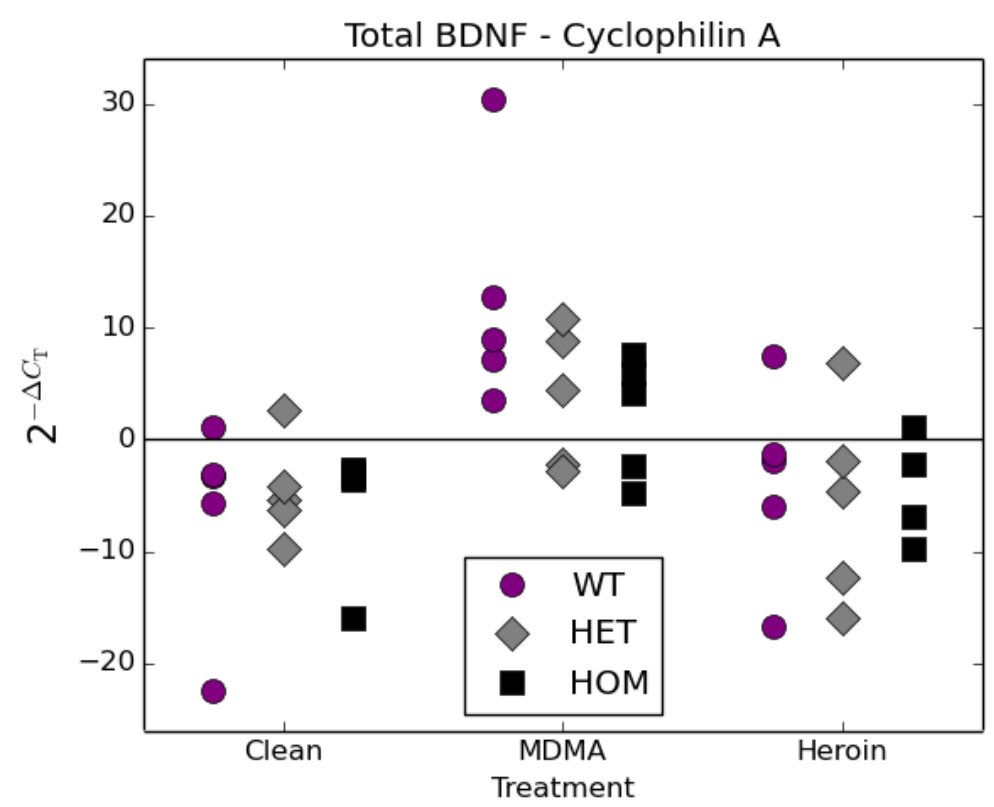

Figure A.5: $2^{-\Delta C_{\mathrm{T}}}$ values for total BDNF against Cyclophilin A. 


\begin{tabular}{|c|c|c|}
\hline \multicolumn{3}{|c|}{ Cyclophilin A } \\
\hline \hline Clean \\
\hline HOM & HET & WT \\
\hline-2.96 & -5.31 & -3.22 \\
\hline-3.57 & -6.34 & 1.18 \\
\hline-2.60 & -9.71 & -22.47 \\
\hline-15.90 & 2.57 & -3.16 \\
\hline-3.20 & -4.11 & -5.68 \\
\hline \hline MDMA \\
\hline HOM & HET & WT \\
\hline 6.02 & -2.14 & 30.48 \\
\hline 4.17 & -2.83 & 12.73 \\
\hline-2.34 & 8.88 & 7.11 \\
\hline 7.62 & 4.50 & 3.58 \\
\hline-4.71 & 10.78 & 9.03 \\
\hline \hline Heroin \\
\hline HOM & HET & WT \\
\hline-6.92 & 6.92 & 7.44 \\
\hline-9.75 & -12.38 & -16.74 \\
\hline 1.10 & -4.66 & -1.87 \\
\hline-2.22 & -15.94 & -1.27 \\
\hline- & -1.90 & -6.00 \\
\hline
\end{tabular}

(a)

\begin{tabular}{|c|c|c|}
\hline \multicolumn{3}{|c|}{ HPRT } \\
\hline \hline Clean \\
\hline HOM & HET & WT \\
\hline-42.96 & -82.14 & -83.87 \\
\hline-113.77 & -131.60 & -76.64 \\
\hline-50.04 & -111.43 & -269.66 \\
\hline-334.30 & -32.90 & -50.74 \\
\hline-71.51 & -84.45 & -122.79 \\
\hline \hline MDMA \\
\hline HOM & HET & WT \\
\hline-3.22 & -25.55 & -1.70 \\
\hline-4.91 & -45.41 & -3.96 \\
\hline-23.67 & -2.39 & -2.75 \\
\hline-2.67 & -24.00 & -35.51 \\
\hline-91.77 & -2.72 & -2.68 \\
\hline \hline Heroin & \\
\hline HOM & HET & WT \\
\hline-81.29 & -2.80 & -2.08 \\
\hline-152.75 & -79.34 & -205.07 \\
\hline-7.52 & -39.81 & -14.62 \\
\hline-15.45 & -106.15 & -18.77 \\
\hline- & -15.78 & -108.76 \\
\hline
\end{tabular}

(b)

Table A.5: $2^{-\Delta C_{\mathrm{T}}}$ values for total BDNF against Cyclophilin A (a) and $\operatorname{HPRT}(b)$. 


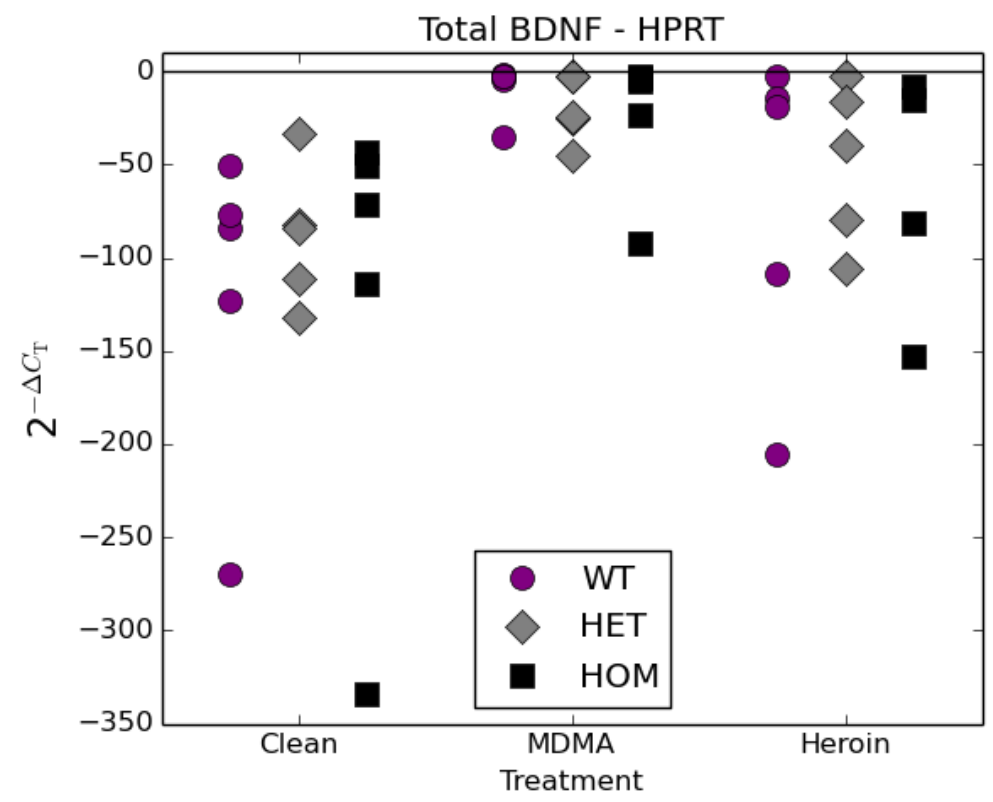

Figure A.6: $2^{-\Delta C_{\mathrm{T}}}$ values for total BDNF against HPRT.

\section{A.3 Section 3: BDNF Transcript III}

Relative expression of BDNF transcript III (against Cyclophilin A and HPRT) was described in Chapter 4.

- $\Delta C_{\mathrm{T}}$ values were calculated for each sample $\left(\Delta C_{\mathrm{T}}=\left(C_{\mathrm{T}}\right.\right.$ BDNF III $)$ $\left(C_{\mathrm{T}}\right.$ Cyc A $)$ ) (see Figure A.7), and $\left(\Delta C_{\mathrm{T}}=\left(C_{\mathrm{T}}\right.\right.$ BDNF III $)-\left(C_{\mathrm{T}}\right.$ HPRT $\left.)\right)$ (see Figure A.8); raw datum values are given in Table A.6.

- Each $\Delta C_{T}$ value was converted into the negative binary $\log \left(2^{-\Delta C_{T}}\right)$ (see Figures A.9 and A.10); raw datum values are given in Table A.7. 


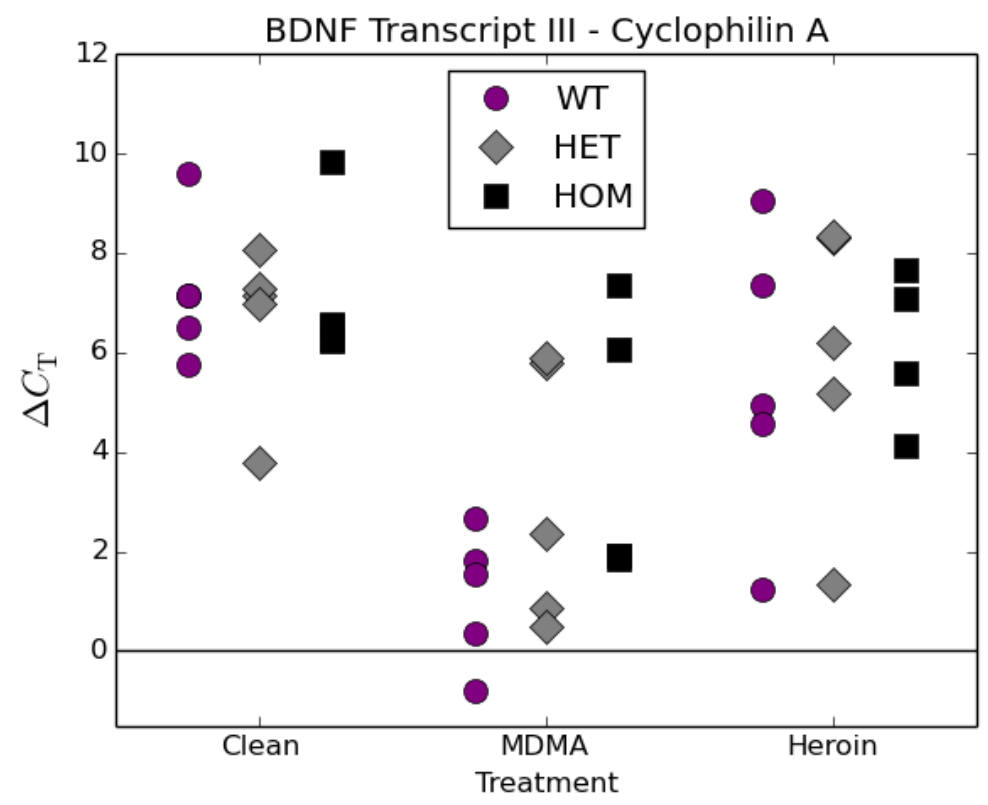

Figure A.7: $\Delta C_{\mathrm{T}}$ values for BDNF transcript III against Cyc A.

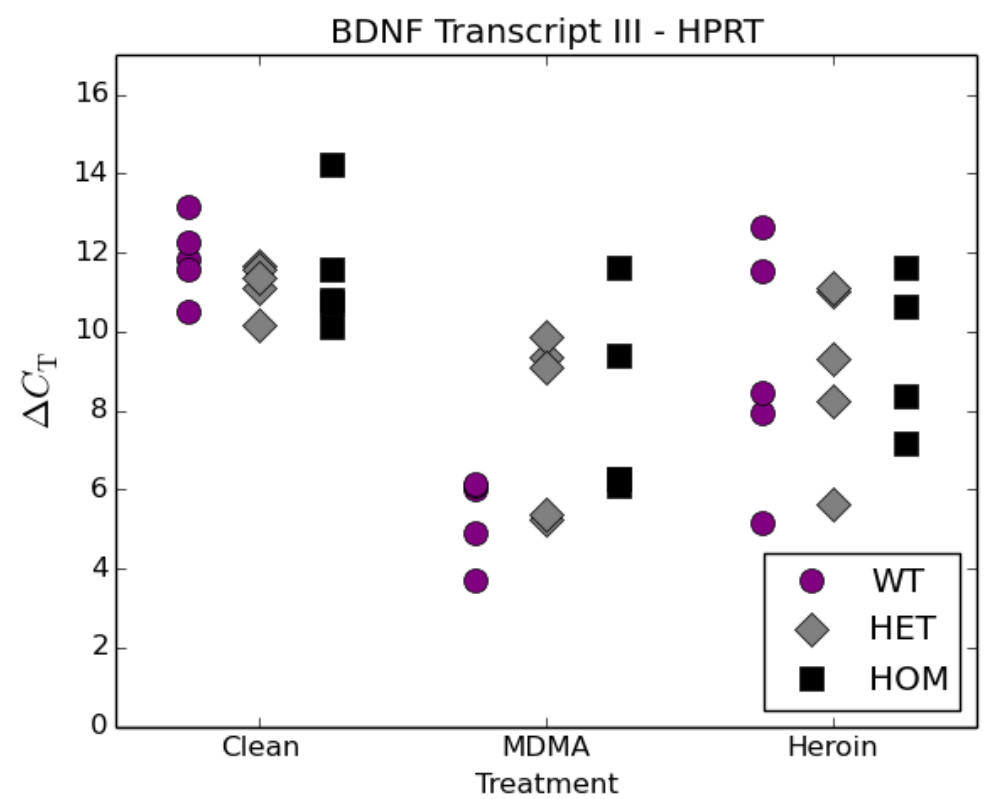

Figure A.8: $\Delta C_{\mathrm{T}}$ values for BDNF transcript III against HPRT. 


\begin{tabular}{|c|c|c|}
\hline \multicolumn{3}{|c|}{ Cyclophilin A } \\
\hline \hline Clean \\
\hline HOM & HET & WT \\
\hline 6.27 & 7.16 & 7.15 \\
\hline 6.58 & 7.29 & 5.76 \\
\hline 6.55 & 8.06 & 9.59 \\
\hline 9.84 & 3.78 & 6.50 \\
\hline 6.25 & 6.99 & 7.16 \\
\hline \hline MDMA \\
\hline HOM & HET & WT \\
\hline 1.85 & 5.78 & -0.8 \\
\hline 1.91 & 5.88 & 0.35 \\
\hline 6.07 & 0.86 & 1.83 \\
\hline 1.91 & 2.35 & 2.68 \\
\hline 7.35 & 0.50 & 1.54 \\
\hline \hline Heroin \\
\hline HOM & HET & WT \\
\hline 7.07 & 1.35 & 1.23 \\
\hline 7.66 & 8.32 & 9.05 \\
\hline 4.12 & 6.21 & 4.96 \\
\hline 5.58 & 8.36 & 4.56 \\
\hline- & 5.19 & 7.36 \\
\hline
\end{tabular}

(a)

\begin{tabular}{|c|c|c|}
\hline \multicolumn{3}{|c|}{ HPRT } \\
\hline \hline Clean \\
\hline HOM & HET & WT \\
\hline 10.13 & 11.11 & 11.85 \\
\hline 11.58 & 11.67 & 12.26 \\
\hline 10.81 & 11.58 & 13.17 \\
\hline 14.24 & 10.18 & 10.5 \\
\hline 10.73 & 11.35 & 11.59 \\
\hline \hline MDMA \\
\hline HOM & HET & WT \\
\hline 6.12 & 9.35 & 4.9 \\
\hline 6.26 & 9.88 & 6.01 \\
\hline 9.41 & 5.26 & 6.12 \\
\hline 6.26 & 9.1 & 3.71 \\
\hline 11.64 & 5.37 & 6.13 \\
\hline \hline Heroin \\
\hline HOM & HET & WT \\
\hline 10.62 & 5.62 & 5.18 \\
\hline 11.63 & 11 & 12.66 \\
\hline 7.17 & 9.31 & 7.93 \\
\hline 8.38 & 11.09 & 8.45 \\
\hline- & 8.25 & 11.54 \\
\hline
\end{tabular}

(b)

Table A.6: $\Delta C_{\mathrm{T}}$ values for BDNF transcript III against Cyclophilin A (a) and HPRT (b). 


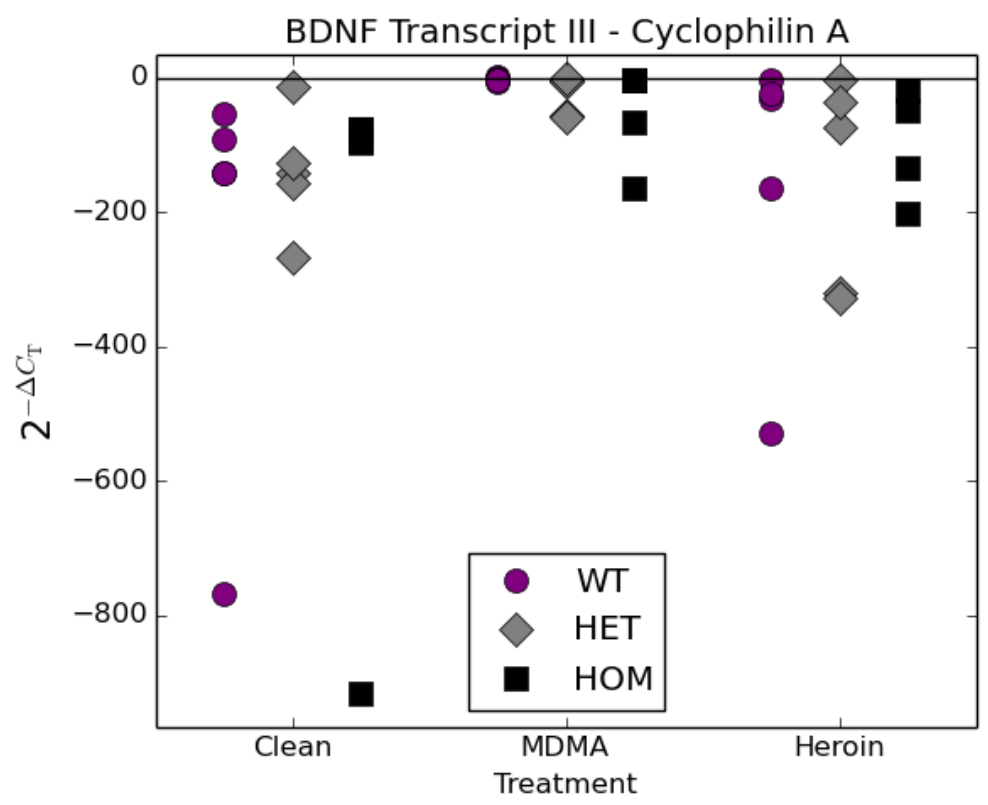

Figure A.9: $2^{-\Delta C_{\mathrm{T}}}$ values for BDNF transcript III against Cyc A.

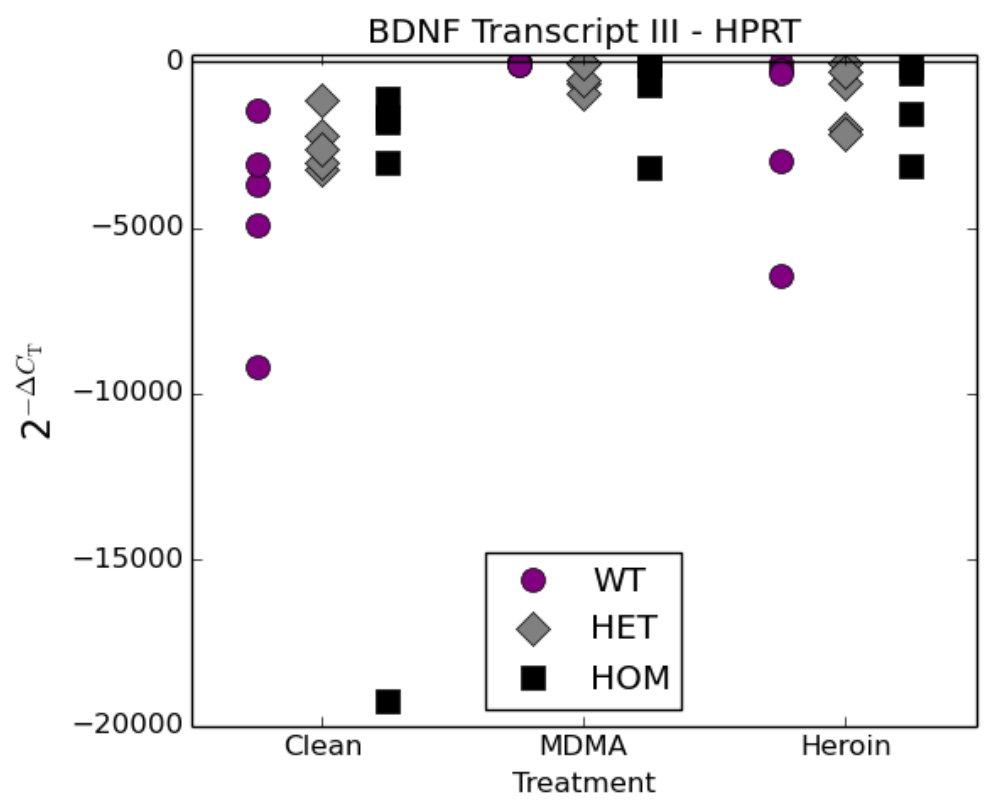

Figure A.10: $2^{-\Delta C_{\mathrm{T}}}$ values for BDNF transcript III against HPRT. 


\begin{tabular}{|c|c|c|}
\hline \multicolumn{3}{|c|}{ Cyclophilin A } \\
\hline \hline Clean \\
\hline HOM & HET & WT \\
\hline-76.90 & -142.52 & -141.53 \\
\hline-95.67 & -156.50 & -54.19 \\
\hline-93.38 & -265.95 & -768.02 \\
\hline-916.51 & -13.74 & -90.20 \\
\hline-75.85 & -127.12 & -142.52 \\
\hline \hline MDMA & \\
\hline HOM & HET & WT \\
\hline-3.59 & -54.76 & 1.74 \\
\hline-3.75 & -58.69 & -1.27 \\
\hline-66.95 & -1.81 & -3.54 \\
\hline-3.76 & -5.08 & -6.41 \\
\hline-163.14 & -1.41 & -2.90 \\
\hline \hline Heroin & \\
\hline HOM & HET & WT \\
\hline-133.90 & -2.54 & -2.34 \\
\hline-201.55 & -319.57 & -528.22 \\
\hline-17.39 & -74.03 & -31.02 \\
\hline-47.84 & -327.42 & -23.59 \\
\hline- & -36.50 & -163.71 \\
\hline
\end{tabular}

(a)

\begin{tabular}{|c|c|c|}
\hline \multicolumn{3}{|c|}{ HPRT } \\
\hline \hline Clean \\
\hline HOM & HET & WT \\
\hline-1116.68 & -2202.61 & -3691.52 \\
\hline-3050.86 & -3247.24 & -4904.87 \\
\hline-1795.29 & -3050.86 & -9216.48 \\
\hline-19282.44 & -1160.07 & -1448.15 \\
\hline-1692.57 & -2610.30 & -3082.75 \\
\hline \hline MDMA & & \\
\hline HOM & HET & WT \\
\hline-69.55 & -652.58 & -29.75 \\
\hline-76.64 & -942.27 & -64.22 \\
\hline-677.93 & -38.32 & -69.31 \\
\hline-76.37 & -548.75 & -13.04 \\
\hline-3180.42 & -41.36 & -70.03 \\
\hline \hline Heroin & & \\
\hline HOM & HET & WT \\
\hline-1573.76 & -49.18 & -36.13 \\
\hline-3158.45 & -2048 & -6472.02 \\
\hline-143.51 & -632.53 & -243.03 \\
\hline-333.14 & -2179.83 & -349.71 \\
\hline- & -303.38 & -2967.43 \\
\hline
\end{tabular}

(b)

Table A.7: $2^{-\Delta C_{\mathrm{T}}}$ values for BDNF transcript III against Cyclophilin A (a) and HPRT (b). 


\section{A.4 Section 4: BDNF Transcript IV}

Relative expression of BDNF transcript IV (against Cyclophilin A and HPRT) was described in Chapter 4.

- $\Delta C_{\mathrm{T}}$ values were calculated for each sample $\left(\Delta C_{\mathrm{T}}=\left(C_{\mathrm{T}}\right.\right.$ BDNF IV) $\left(C_{\mathrm{T}}\right.$ Cyc A $)$ ) (see Figure A.11), and $\left(\Delta C_{\mathrm{T}}=\left(C_{\mathrm{T}}\right.\right.$ BDNF IV $)-\left(C_{\mathrm{T}}\right.$ HPRT $\left.)\right)$ (see Figure A.12); raw datum values are given in Table A.8.

- Each $\Delta C_{\mathrm{T}}$ value was converted into the negative binary $\log \left(2^{-\Delta C_{\mathrm{T}}}\right)$ (see Figures A.13 and A.14); raw datum values are given in Table A.9.

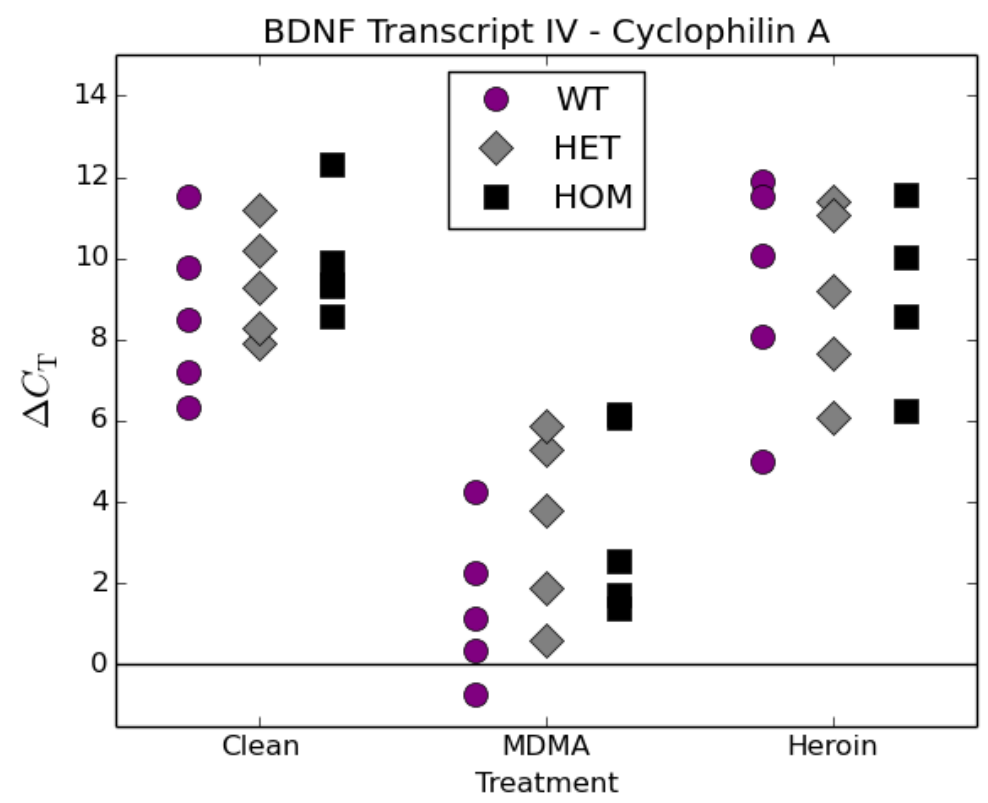

Figure A.11: $\Delta C_{\mathrm{T}}$ values for BDNF transcript IV against Cyc A. 


\begin{tabular}{|c|c|c|}
\hline \multicolumn{3}{|c|}{ Cyclophilin A } \\
\hline \hline Clean \\
\hline HOM & HET & WT \\
\hline 9.38 & 7.93 & 7.2 \\
\hline 9.31 & 8.28 & 6.34 \\
\hline 8.58 & 11.19 & 11.54 \\
\hline 12.31 & 9.3 & 8.51 \\
\hline 9.9 & 10.21 & 9.79 \\
\hline \hline MDMA \\
\hline HOM & HET & WT \\
\hline 1.4 & 5.3 & -0.72 \\
\hline 2.57 & 5.87 & 0.35 \\
\hline 6.17 & 1.88 & 2.28 \\
\hline 1.74 & 3.81 & 4.26 \\
\hline 6.07 & 0.62 & 1.14 \\
\hline \hline Heroin \\
\hline HOM & HET & WT \\
\hline 10.01 & 6.07 & 5.02 \\
\hline 11.57 & 11.42 & 11.89 \\
\hline 6.26 & 9.21 & 8.07 \\
\hline 8.58 & 11.05 & 11.51 \\
\hline- & 7.68 & 10.09 \\
\hline
\end{tabular}

(a)

\begin{tabular}{|c|c|c|}
\hline \multicolumn{3}{|c|}{ HPRT } \\
\hline \hline Clean \\
\hline HOM & HET & WT \\
\hline 13.24 & 11.88 & 11.91 \\
\hline 14.3 & 12.66 & 12.84 \\
\hline 12.84 & 14.71 & 15.12 \\
\hline 16.71 & 15.7 & 12.52 \\
\hline 14.38 & 14.57 & 14.22 \\
\hline \hline MDMA \\
\hline HOM & HET & WT \\
\hline 5.68 & 8.88 & 4.98 \\
\hline 6.92 & 9.87 & 6.01 \\
\hline 9.51 & 6.29 & 6.57 \\
\hline 6.08 & 10.57 & 11.25 \\
\hline 10.35 & 5.49 & 5.73 \\
\hline \hline Heroin \\
\hline HOM & HET & WT \\
\hline 13.57 & 10.35 & 8.97 \\
\hline 15.54 & 14.1 & 15.51 \\
\hline 9.31 & 12.3 & 11.04 \\
\hline 11.38 & 13.79 & 15.4 \\
\hline- & 10.74 & 14.27 \\
\hline
\end{tabular}

(b)

Table A.8: $\Delta C_{\mathrm{T}}$ values for BDNF transcript IV against Cyclophilin A (a) and HPRT (b). 


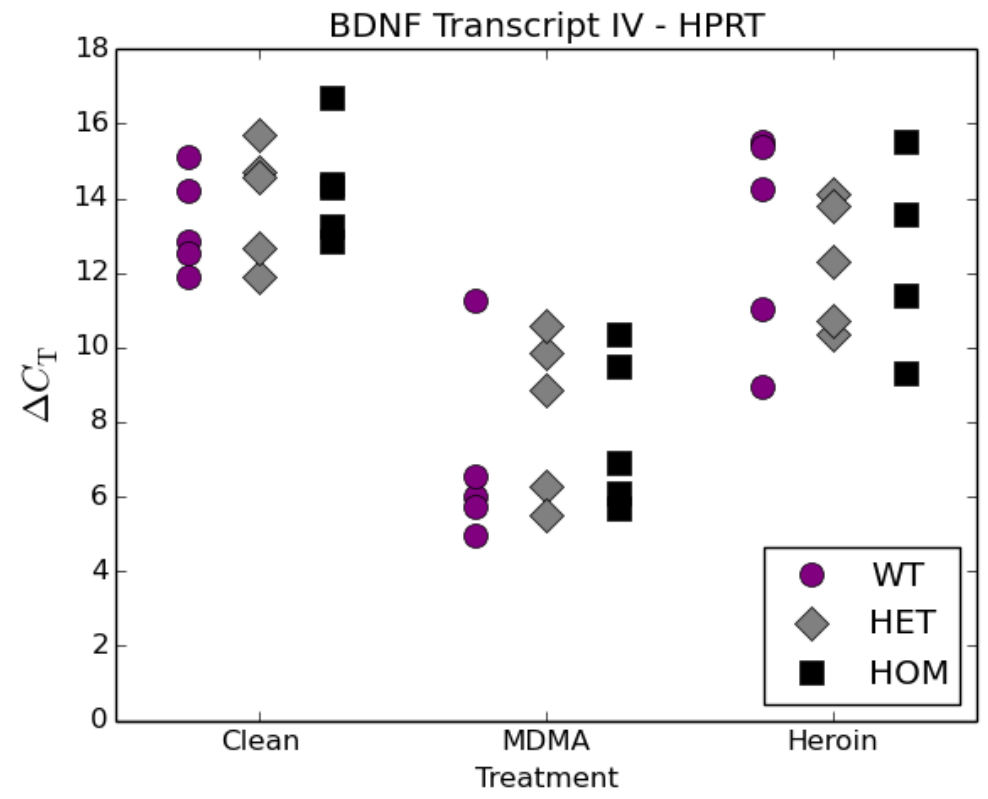

Figure A.12: $\Delta C_{\mathrm{T}}$ values for BDNF transcript IV against HPRT.

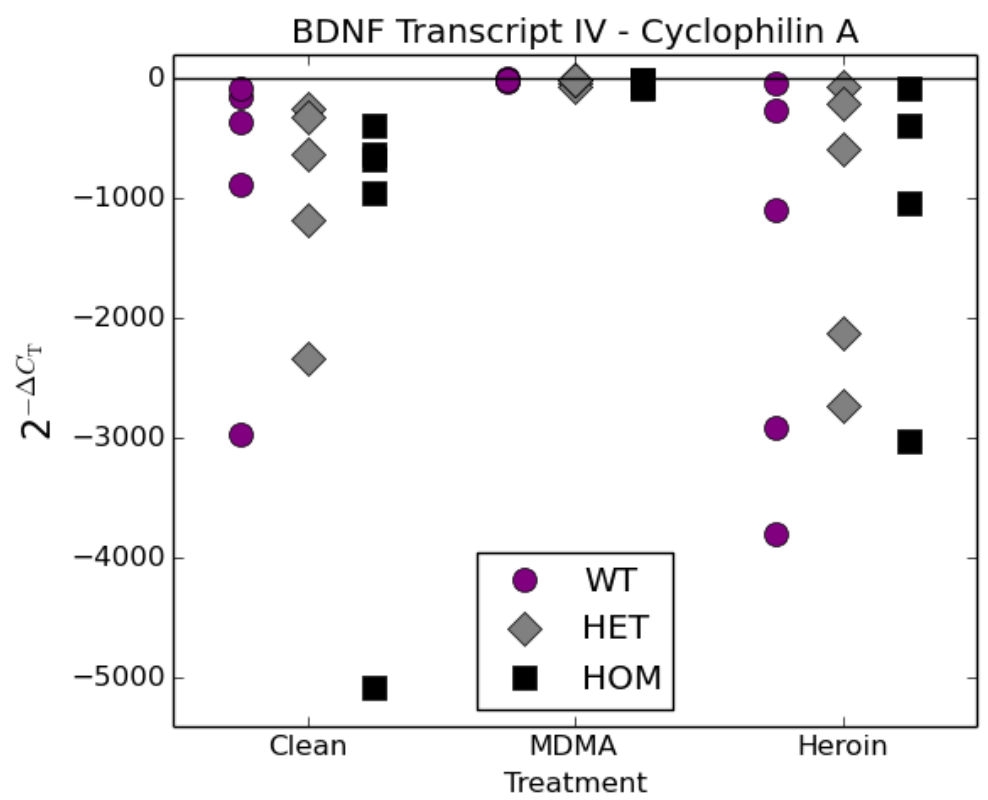

Figure A.13: $2^{-\Delta C_{\mathrm{T}}}$ values for BDNF transcript IV against Cyc A. 


\begin{tabular}{|c|c|c|}
\hline \multicolumn{3}{|c|}{ Cyclophilin A } \\
\hline \hline Clean \\
\hline HOM & HET & WT \\
\hline-666.29 & -243.03 & -147.03 \\
\hline-632.53 & -310.83 & -81.01 \\
\hline-381.36 & -2328.20 & -2967.43 \\
\hline-5077.84 & -628.17 & -364.56 \\
\hline-955.43 & -1184.45 & -882.22 \\
\hline \hline MDMA & \\
\hline HOM & HET & WT \\
\hline-2.64 & -39.4 & 1.64 \\
\hline-5.92 & -58.28 & -1.27 \\
\hline-71.75 & -3.68 & -4.86 \\
\hline-3.33 & -14.03 & -19.16 \\
\hline-66.95 & -1.53 & -2.2 \\
\hline \hline Heroin & & \\
\hline HOM & HET & WT \\
\hline-1031.12 & -67.18 & -32.45 \\
\hline-3029.79 & -2730.6 & -3795.30 \\
\hline-76.64 & -590.18 & -267.8 \\
\hline-382.68 & -2120.22 & -2916.45 \\
\hline- & -205.07 & -1086.14 \\
\hline
\end{tabular}

(a)

\begin{tabular}{|c|c|c|}
\hline \multicolumn{3}{|c|}{ HPRT } \\
\hline \multicolumn{3}{|l|}{ Clean } \\
\hline HOM & HET & WT \\
\hline-9674.69 & -3756.05 & -3834.97 \\
\hline-20171.07 & -6449.63 & -7332.05 \\
\hline-7332.05 & -26708.29 & -35610.13 \\
\hline-106833.16 & -53047.61 & -5853.16 \\
\hline-21321.18 & -24322.43 & -19083 \\
\hline \multicolumn{3}{|l|}{ MDMA } \\
\hline HOM & HET & WT \\
\hline-51.09 & -469.51 & -31.56 \\
\hline-121.1 & -935.76 & -64.22 \\
\hline-726.59 & -77.98 & -95.01 \\
\hline-67.65 & -1514.89 & -2435.5 \\
\hline-1305.15 & -44.94 & -53.08 \\
\hline \multicolumn{3}{|l|}{ Heroin } \\
\hline HOM & HET & WT \\
\hline-12119.14 & -1300.63 & -501.46 \\
\hline-47478.94 & -17499.18 & -46501.83 \\
\hline-632.53 & -5042.77 & -2098.29 \\
\hline-2665.15 & -14115.57 & -43237.64 \\
\hline- & -1704.34 & -19687.61 \\
\hline
\end{tabular}

(b)

Table A.9: $2^{-\Delta C_{\mathrm{T}}}$ values for BDNF transcript IV against Cyclophilin A (a) and HPRT (b). 


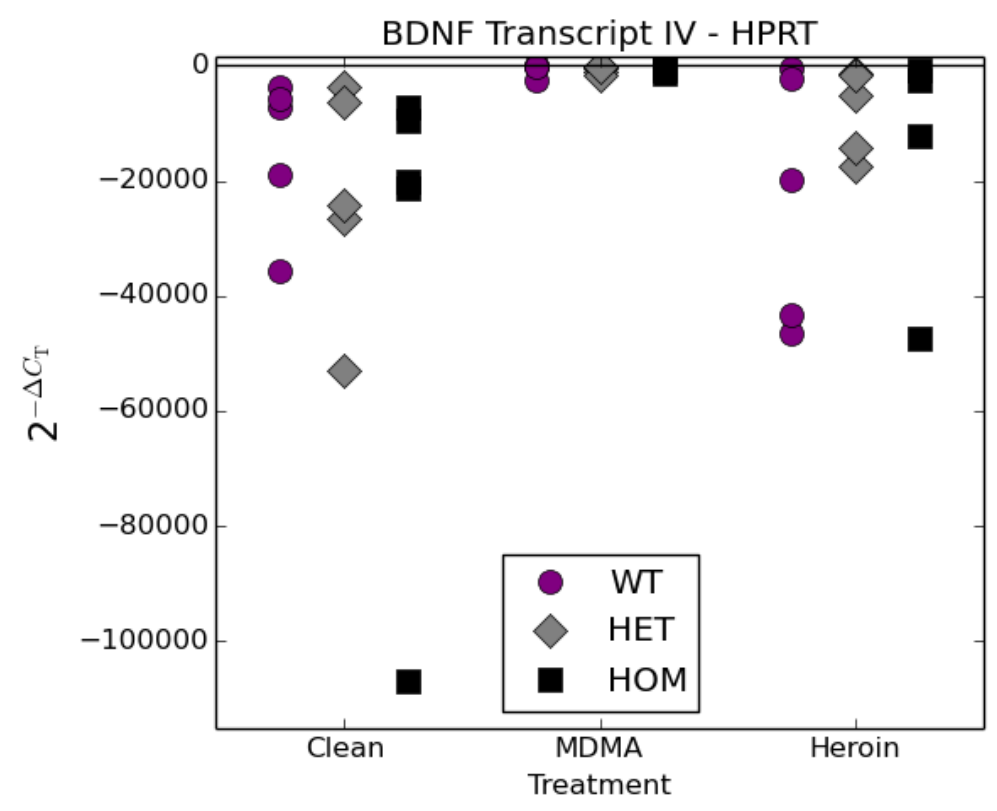

Figure A.14: $2^{-\Delta C_{\mathrm{T}}}$ values for BDNF transcript IV against HPRT.

\section{A.5 Section 5: BDNF Transcript VI}

Relative expression of BDNF transcript VI (against Cyclophilin A and HPRT) was described in Chapter 4.

- $\Delta C_{\mathrm{T}}$ values were calculated for each sample $\left(\Delta C_{\mathrm{T}}=\left(C_{\mathrm{T}}\right.\right.$ BDNF VI) $\left(C_{\mathrm{T}}\right.$ Cyc A $)$ (see Figure A.15), and $\left(\Delta C_{\mathrm{T}}=\left(C_{\mathrm{T}} \mathrm{BDNF}\right.\right.$ VI $\left.)-\left(C_{\mathrm{T}} \mathrm{HPRT}\right)\right)$ (see Figure A.16); raw datum values are given in Table A.10.

- Each $\Delta C_{\mathrm{T}}$ value was converted into the negative binary $\log \left(2^{-\Delta C_{\mathrm{T}}}\right)$ (see Figures A.17 and A.18); raw datum values are given in Table A.11. 


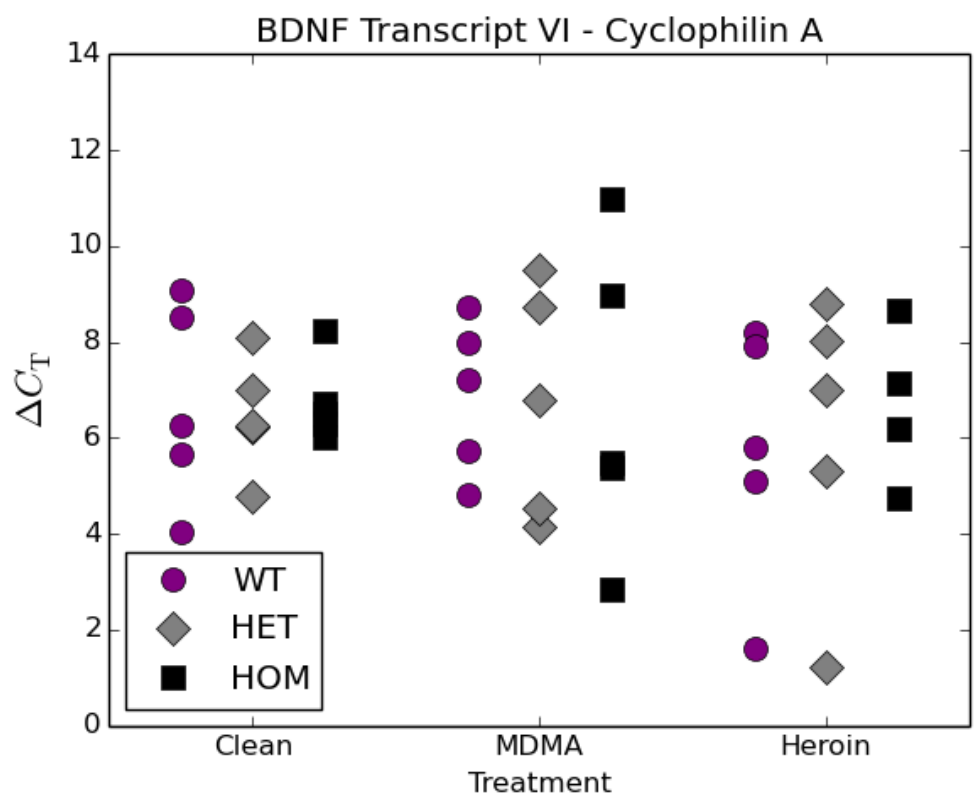

Figure A.15: $\Delta C_{\mathrm{T}}$ values for BDNF transcript VI against Cyc $\mathrm{A}$.

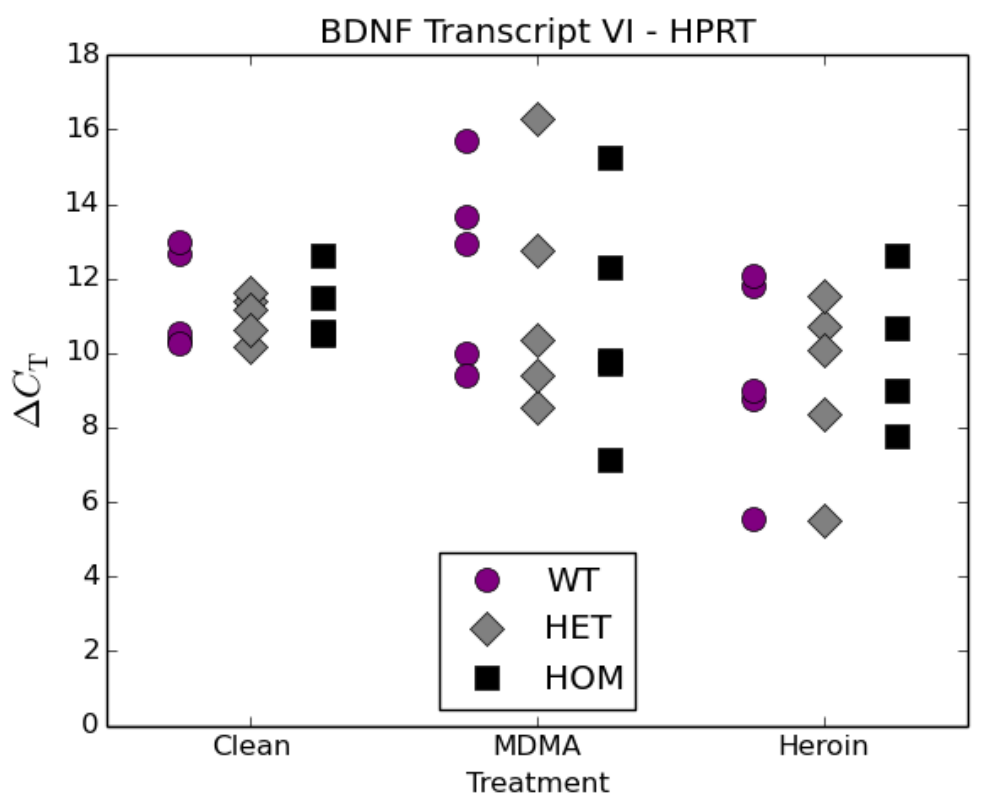

Figure A.16: $\Delta C_{\mathrm{T}}$ values for BDNF transcript VI against HPRT. 


\begin{tabular}{|c|c|c|}
\hline \multicolumn{3}{|c|}{ Cyclophilin A } \\
\hline \hline Clean \\
\hline HOM & HET & WT \\
\hline 6.71 & 6.21 & 5.65 \\
\hline 6.51 & 7.01 & 4.04 \\
\hline 6.28 & 8.1 & 9.07 \\
\hline 8.22 & 4.79 & 6.26 \\
\hline 6.01 & 6.25 & 8.53 \\
\hline \hline MDMA \\
\hline HOM & HET & WT \\
\hline 2.84 & 6.8 & 7.23 \\
\hline 5.47 & 8.74 & 7.99 \\
\hline 8.98 & 4.15 & 5.71 \\
\hline 5.37 & 9.52 & 8.72 \\
\hline 10.98 & 4.53 & 4.81 \\
\hline \hline Heroin \\
\hline HOM & HET & WT \\
\hline 7.13 & 1.23 & 1.6 \\
\hline 8.64 & 8.03 & 8.19 \\
\hline 4.74 & 7.00 & 5.8 \\
\hline 6.2 & 8.79 & 5.11 \\
\hline- & 5.3 & 7.92 \\
\hline
\end{tabular}

(a)

\begin{tabular}{|c|c|c|}
\hline \multicolumn{3}{|c|}{ HPRT } \\
\hline \hline Clean \\
\hline HOM & HET & WT \\
\hline 10.57 & 10.16 & 10.36 \\
\hline 11.51 & 11.39 & 10.54 \\
\hline 10.54 & 11.62 & 12.66 \\
\hline 12.61 & 11.19 & 10.26 \\
\hline 10.49 & 10.61 & 12.96 \\
\hline \hline MDMA \\
\hline HOM & HET & WT \\
\hline 7.12 & 10.38 & 12.92 \\
\hline 9.82 & 12.74 & 13.65 \\
\hline 12.32 & 8.56 & 10.00 \\
\hline 9.71 & 16.27 & 15.71 \\
\hline 15.27 & 9.4 & 9.4 \\
\hline \hline Heroin \\
\hline HOM & HET & WT \\
\hline 10.69 & 5.51 & 5.55 \\
\hline 12.61 & 10.71 & 11.81 \\
\hline 7.78 & 10.09 & 8.77 \\
\hline 9.00 & 11.52 & 9.00 \\
\hline- & 8.36 & 12.10 \\
\hline \multicolumn{2}{|c|}{} \\
\hline
\end{tabular}

(b)

Table A.10: $\Delta C_{\mathrm{T}}$ values for BDNF transcript VI against Cyclophilin A (a) and HPRT (b). 


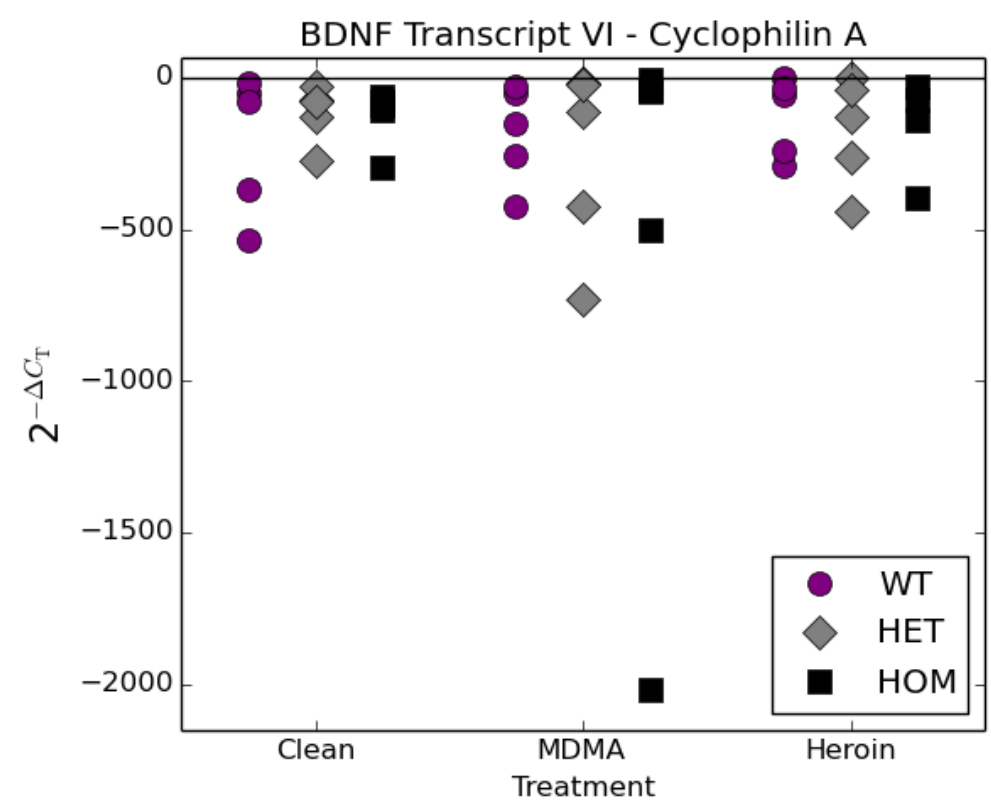

Figure A.17: $2^{-\Delta C_{\mathrm{T}}}$ values for BDNF transcript VI against Cyc A.

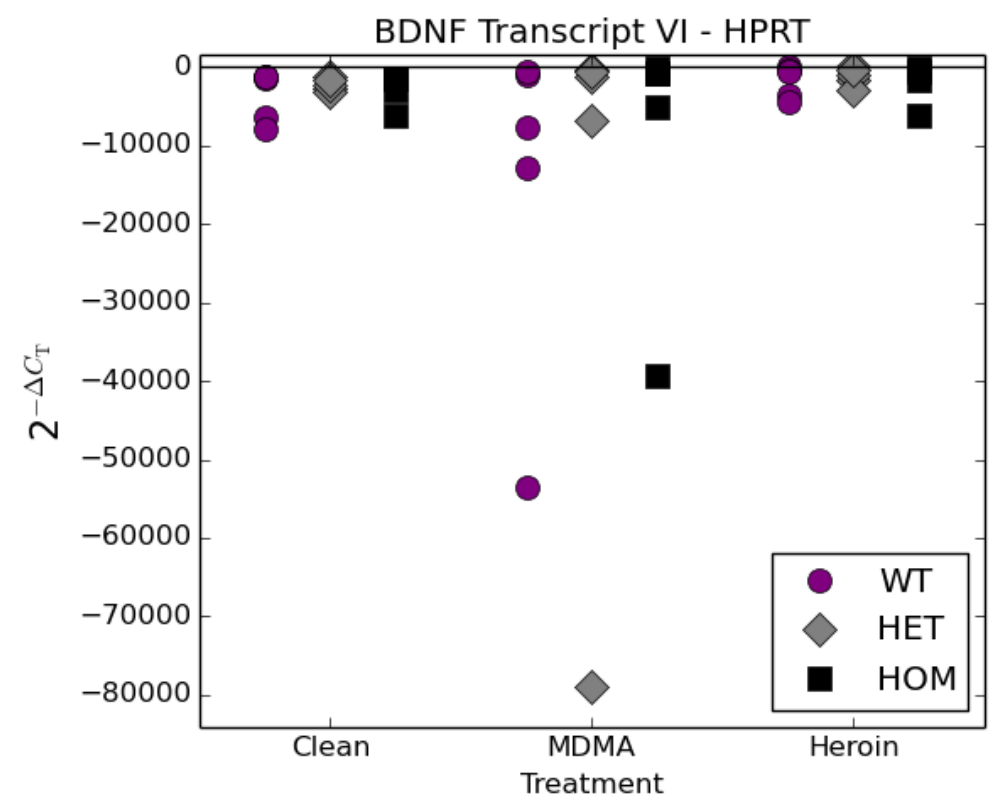

Figure A.18: $2^{-\Delta C_{\mathrm{T}}}$ values for BDNF transcript VI against HPRT. 


\begin{tabular}{|c|c|c|}
\hline \multicolumn{3}{|c|}{ Cyclophilin A } \\
\hline \hline Clean \\
\hline HOM & HET & WT \\
\hline-104.33 & -73.77 & -50.21 \\
\hline-91.14 & -128.89 & -16.39 \\
\hline-77.44 & -273.42 & -537.45 \\
\hline-297.14 & -27.57 & -76.37 \\
\hline-64.22 & -76.11 & -368.37 \\
\hline \hline MDMA \\
\hline HOM & HET & WT \\
\hline-7.16 & -111.43 & -149.60 \\
\hline-44.17 & -426.09 & -254.23 \\
\hline-503.20 & -17.75 & -52.35 \\
\hline-41.21 & -731.64 & -421.68 \\
\hline-2019.80 & -23.023 & -27.95 \\
\hline \hline Heroin & \\
\hline HOM & HET & WT \\
\hline-140.07 & -2.35 & -3.03 \\
\hline-397.55 & -261.38 & -292.04 \\
\hline-26.63 & -127.56 & -55.52 \\
\hline-73.26 & -441.11 & -34.42 \\
\hline- & -39.4 & -241.35 \\
\hline
\end{tabular}

(a)

\begin{tabular}{|c|c|c|}
\hline \multicolumn{3}{|c|}{ HPRT } \\
\hline \hline \multicolumn{1}{|l|}{ Clean } \\
\hline HOM & HET & WT \\
\hline-1514.89 & -1140.14 & -1309.68 \\
\hline-2906.36 & -2674.40 & -1483.72 \\
\hline-1488.87 & -3136.63 & -6449.63 \\
\hline-6251.56 & -2328.20 & -1226.22 \\
\hline-1433.18 & -1562.89 & -7967.99 \\
\hline \hline MDMA & & \\
\hline HOM & HET & WT \\
\hline-138.62 & -1327.96 & -7750.10 \\
\hline-903.89 & -6841.04 & -12810.15 \\
\hline-5095.47 & -376.11 & -1024 \\
\hline-837.53 & -79023.82 & -53602.03 \\
\hline-39375.21 & -675.59 & -675.59 \\
\hline \hline Heroin & & \\
\hline HOM & HET & WT \\
\hline-1646.29 & -45.41 & -46.85 \\
\hline-6229.93 & -1675.06 & -3578.15 \\
\hline-219.79 & -1089.92 & -435.04 \\
\hline-510.23 & -2936.74 & -510.23 \\
\hline- & -327.42 & -4374.8 \\
\hline
\end{tabular}

(b)

Table A.11: $2^{-\Delta C_{\mathrm{T}}}$ values for BDNF transcript VI against Cyclophilin A (a) and HPRT (b). 


\section{A.6 Section 6: BDNF Transcript IXa}

Relative expression of BDNF transcript IXa (against Cyclophilin A and HPRT) was described in Chapter 4.

- $\Delta C_{\mathrm{T}}$ values were calculated for each sample $\left(\Delta C_{\mathrm{T}}=\left(C_{\mathrm{T}}\right.\right.$ BDNF IXa) - $\left(C_{\mathrm{T}}\right.$ Cyc A $)$ ) (see Figure A.19), and $\left(\Delta C_{\mathrm{T}}=\left(C_{\mathrm{T}}\right.\right.$ BDNF IXa) - $\left(C_{\mathrm{T}}\right.$ HPRT)) (see Figure A.20); raw datum values are given in Table A.12.

- Each $\Delta C_{\mathrm{T}}$ value was converted into the negative binary $\log \left(2^{-\Delta C_{\mathrm{T}}}\right)$ (see Figures A.21 and A.22); raw datum values are given in Table A.13.

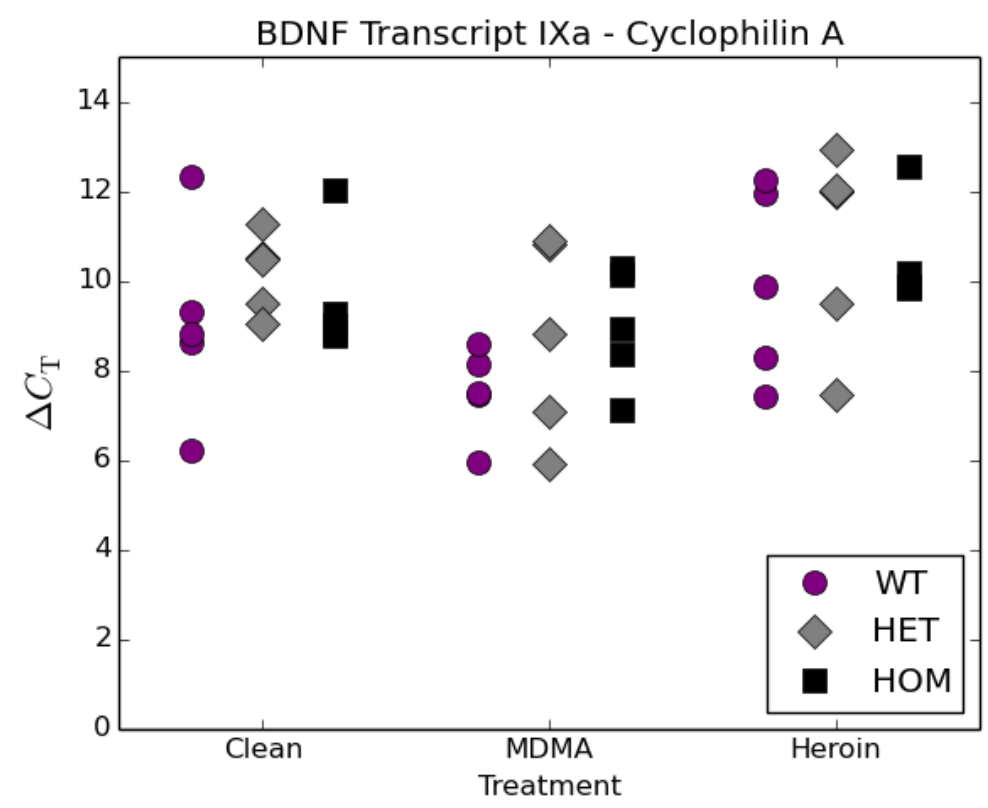

Figure A.19: $\Delta C_{\mathrm{T}}$ values for BDNF transcript IXa against Cyc A. 


\begin{tabular}{|c|c|c|}
\hline \multicolumn{3}{|c|}{ Cyclophilin A } \\
\hline \hline Clean \\
\hline HOM & HET & WT \\
\hline 9.26 & 10.51 & 8.63 \\
\hline 8.80 & 9.52 & 6.21 \\
\hline 8.84 & 10.48 & 12.33 \\
\hline 12.01 & 9.05 & 9.3 \\
\hline 9.02 & 11.27 & 8.82 \\
\hline \hline MDMA \\
\hline HOM & HET & WT \\
\hline 7.1 & 10.82 & 7.47 \\
\hline 8.92 & 10.90 & 5.94 \\
\hline 10.16 & 7.09 & 8.13 \\
\hline 8.38 & 8.81 & 8.58 \\
\hline 10.28 & 5.93 & 7.48 \\
\hline \hline Heroin \\
\hline HOM & HET & WT \\
\hline 10.18 & 7.47 & 7.42 \\
\hline 12.56 & 12.00 & 11.95 \\
\hline 9.83 & 12.92 & 9.87 \\
\hline 9.89 & 12.01 & 8.3 \\
\hline- & 9.5 & 12.25 \\
\hline
\end{tabular}

(a)

\begin{tabular}{|c|c|c|}
\hline \multicolumn{3}{|c|}{ HPRT } \\
\hline \hline Clean \\
\hline HOM & HET & WT \\
\hline 13.12 & 14.46 & 13.34 \\
\hline 13.79 & 13.89 & 12.71 \\
\hline 13.1 & 14.00 & 15.92 \\
\hline 16.41 & 15.45 & 13.31 \\
\hline 13.50 & 15.63 & 13.25 \\
\hline \hline MDMA \\
\hline HOM & HET & WT \\
\hline 11.38 & 14.39 & 13.16 \\
\hline 13.28 & 14.9 & 11.6 \\
\hline 13.5 & 11.5 & 12.42 \\
\hline 12.72 & 15.56 & 15.57 \\
\hline 14.57 & 10.8 & 12.08 \\
\hline \hline Heroin \\
\hline HOM & HET & WT \\
\hline 13.74 & 11.74 & 11.37 \\
\hline 16.53 & 14.68 & 15.57 \\
\hline 12.87 & 16.02 & 12.84 \\
\hline 12.69 & 14.75 & 12.19 \\
\hline- & 12.56 & 16.43 \\
\hline
\end{tabular}

(b)

Table A.12: $\Delta C_{\mathrm{T}}$ values for BDNF transcript IXa against Cyclophilin A (a) and HPRT (b). 


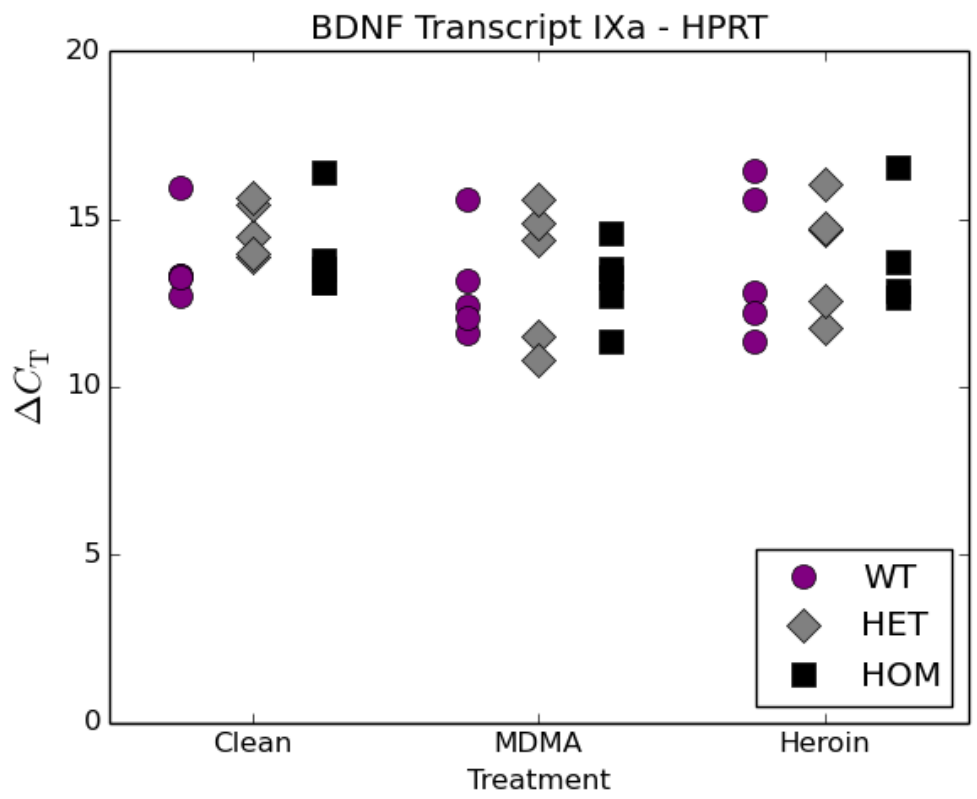

Figure A.20: $\Delta C_{\mathrm{T}}$ values for BDNF transcript IXa against HPRT.

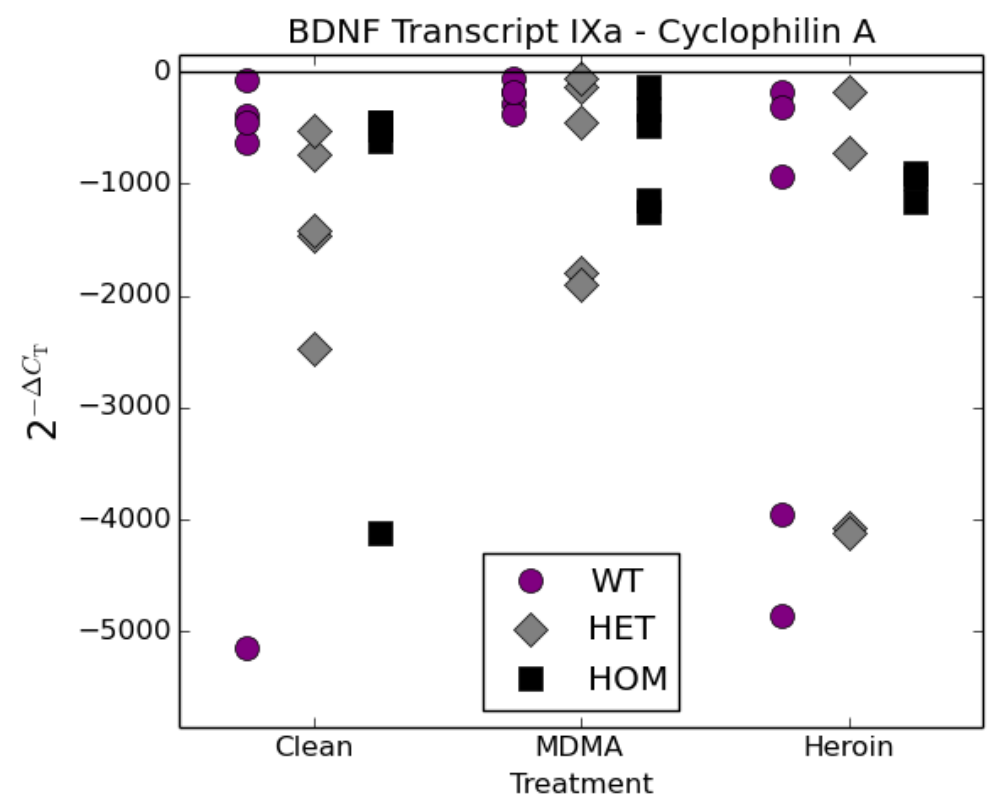

Figure A.21: $2^{-\Delta C_{\mathrm{T}}}$ values for BDNF transcript IXa against Cyc A. 


\begin{tabular}{|c|c|c|}
\hline \multicolumn{3}{|c|}{ Cyclophilin A } \\
\hline \hline Clean \\
\hline HOM & HET & WT \\
\hline-610.99 & -1458.23 & -396.18 \\
\hline-444.18 & -731.64 & -73.77 \\
\hline-456.67 & -1423.28 & -5148.73 \\
\hline-4124.49 & -530.06 & -630.35 \\
\hline-517.35 & -2469.49 & -450.38 \\
\hline \hline MDMA & \\
\hline HOM & HET & WT \\
\hline-137.19 & -1801.52 & -176.68 \\
\hline-484.38 & -1904.24 & -61.39 \\
\hline-1140.14 & -136.24 & -279.17 \\
\hline-332 & -447.27 & -381.36 \\
\hline-1243.34 & -60.76 & -178.53 \\
\hline \hline Heroin & \\
\hline HOM & HET & WT \\
\hline-1160.07 & -176.68 & -171.25 \\
\hline-6038.61 & -4081.83 & -3956.48 \\
\hline-907.03 & -7750.10 & -932.53 \\
\hline-945.54 & -4124.49 & -315.17 \\
\hline- & -724.08 & -4854.14 \\
\hline
\end{tabular}

(a)

\begin{tabular}{|c|c|c|}
\hline \multicolumn{3}{|c|}{ HPRT } \\
\hline \hline Clean \\
\hline HOM & HET & WT \\
\hline-8871.73 & -22536.88 & -10333.20 \\
\hline-14164.58 & -15181.22 & -6677.07 \\
\hline-8779.97 & -16327.32 & -61786.33 \\
\hline-86775.49 & -44762.41 & -10120.55 \\
\hline-11545.16 & -50710.61 & -9741.98 \\
\hline \hline MDMA & HET & WT \\
\hline HOM & HO \\
\hline-2655.93 & -21469.49 & -9152.82 \\
\hline-9912.27 & -30573.63 & -3093.45 \\
\hline-11545.16 & -2886.29 & -5461.19 \\
\hline-6746.86 & -48308.85 & -48476.57 \\
\hline-24238.28 & -1782.89 & -4314.57 \\
\hline \hline Heroin & & \\
\hline HOM & HET & WT \\
\hline-13634.74 & -3420.52 & -2646.74 \\
\hline-94629.34 & -26158.64 & -48476.57 \\
\hline-7486.10 & -66220.95 & -7306.68 \\
\hline-6585.15 & -27459.16 & -4672.57 \\
\hline \multicolumn{2}{|c|}{-6017.71} & -87986.83 \\
\hline
\end{tabular}

(b)

Table A.13: Converted $2^{-\Delta C_{\mathrm{T}}}$ values for BDNF transcript IXa against Cyclophilin A (a) and HPRT (b). 


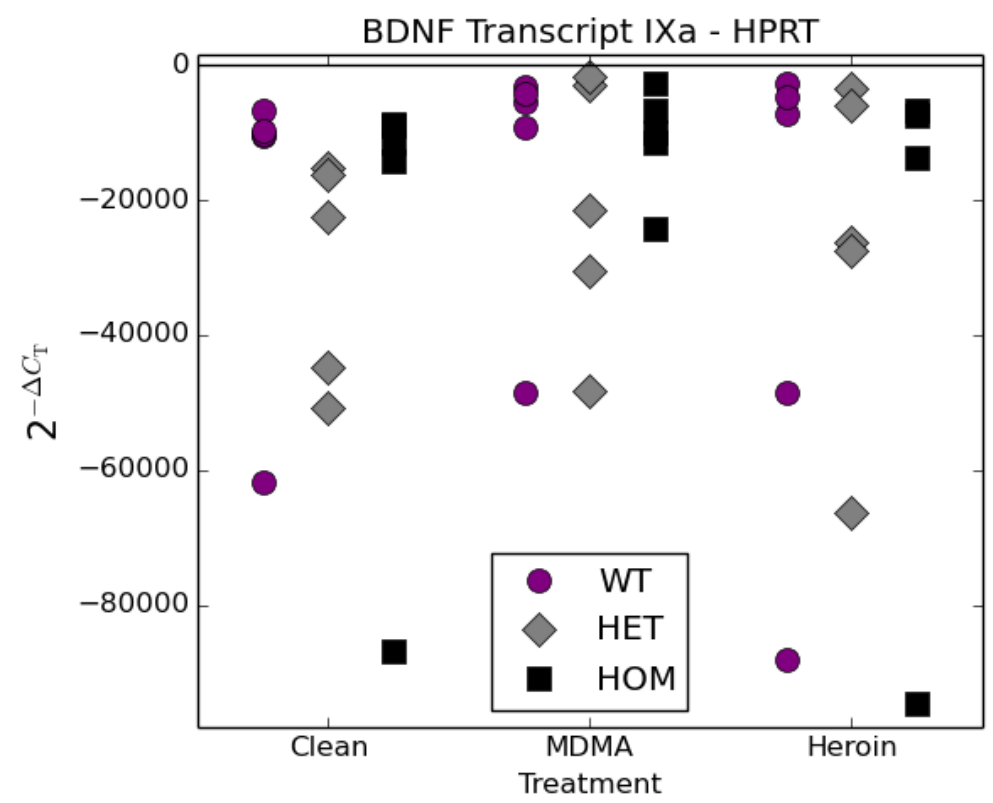

Figure A.22: $2^{-\Delta C_{\mathrm{T}}}$ values for BDNF transcript IXa against HPRT. 


\section{Bibliography}

Ahmed, S. H. (1998). Transition from moderate to excessive drug intake: Change in hedonic set point [Journal Article]. Science, 282(5387), 298300.

Ahmed, S. H. (2010). Validation crisis in animal models of drug addiction: Beyond non-disordered drug use toward drug addiction [Journal Article]. Neuroscience and Biobehavioral Reviews, 35(2), 172-184.

Ahmed, S. H. (2012). The science of making drug-addicted animals [Journal Article]. Neuroscience, 211, 107-125.

Aid, T., Kazantseva, A., Piirsoo, M., Palm, K., \& Timmusk, T. (2007). Mouse and rat BDNF gene structure and expression revisited [Journal Article]. Journal of Neuroscience Research, 85(3), 525-535.

Alex, K. D., \& Pehek, E. A. (2007). Pharmacologic mechanisms of serotonergic regulation of dopamine neurotransmission [Journal Article]. Pharmacology \& Therapeutics, 113(2), 296-320.

American Psychiatric Association. (2013). Diagnostic and statistical manual of mental disorders : Dsm-5 [Book]. (5th ed.).

Anden, N., Butcher, S., Corrodi, H., Fuxe, K., \& Ungerstedt, U. (1970). Receptor activity and turnover of dopamine and noradrenaline after neuroleptics. European Journal of Pharmacology, 11(3), 303-314.

Arthurs, J., Lin, J.-Y., Amodeo, L. R., \& Reilly, S. (2012). Reduced palatability in drug-induced taste aversion: Ii. aversive and rewarding unconditioned stimuli. Behavioral Neuroscience, 126(3), 433-444.

Badiani, A., Belin, D., Epstein, D., Calu, D., \& Shaham, Y. (2011). Opiate 
versus psychostimulant addiction: the differences do matter [Journal Article]. Nature Reviews Neuroscience, 12(11), 685-700.

Ball, K., Budreau, D., \& Rebec, G. (2003). Acute effects of 3,4methylenedioxymethamphetamine on striatal single-unit activity and behavior in freely moving rats: differential involvement of dopamine d-1 and d-2 receptors. Brain Research, 994(2), 203-215.

Baumann, M. H., Clark, R. D., Woolverton, W. L., Wee, S., Blough, B. E., \& Rothman, R. B. (2011). In vivo effects of amphetamine analogs reveal evidence for serotonergic inhibition of mesolimbic dopamine transmission in the rat [Journal Article]. Journal of Pharmacology and Experimental Therapeutics, 337(1), 218-225.

Beninger, R. J. (1983). The role of dopamine in locomotor activity and learning [Journal Article]. Brain Research Reviews, 6(2), 173-196.

Benveniste, H., \& Httemeier, P. C. (1990). Microdialysis: Theory and application. Progress in Neurobiology, 35(3), 195 - 215.

Berglind, W. J., See, R. E., Fuchs, R. A., Ghee, S. M., Whitfield, T. W., Jr., Miller, S. W., \& McGinty, J. F. (2007). A BDNF infusion into the medial prefrontal cortex suppresses cocaine seeking in rats. European Journal Of Neuroscience, 26(3), 757-766.

Binder, D. K., \& Scharfman, H. E. (2004). Brain-derived neurotrophic factor [Journal Article]. Growth Factors, 22(3), 123-131.

Bradbury, S., Bird, J., Colussi-Mas, J., Mueller, M., Ricaurte, G., \& Schenk, S. (2014). Acquisition of MDMA self-administration: pharmacokinetic factors and mdma-induced serotonin release. Addiction Biology, 19(5), 874-884.

Bradbury, S., Gittings, D., \& Schenk, S. (2012). Repeated exposure to MDMA and amphetamine: sensitization, cross-sensitization, and response to dopamine d-1- and d-2-like agonists [Journal Article]. Psychopharmacology, 223(4), 389-399.

Bradbury, S., \& Schenk, S. (2011). Serotonin depletion enhances the reinforcing effects of MDMA [Journal Article]. Behavioural Pharmacology, 


\section{2, E56-E56.}

Bromberg-Martin, E. S., Matsumoto, M., \& Hikosaka, O. (2010). Dopamine in motivational control: Rewarding, aversive, and alerting. Neuron, 68(5), 815-834.

Brown, R. M., Short, J. L., \& Lawrence, A. J. (2010). Identification of brain nuclei implicated in cocaine-primed reinstatement of conditioned place preference: A behaviour dissociable from sensitization. PLOS ONE, 5(12).

Bustin, S. A., Benes, V., Nolan, T., \& Pfaffl, M. W. (2005). Quantitative realtime RT-PCR - A Perspective [Journal Article]. Journal of Molecular Endocrinology, 34(3), 597-601.

Calabrese, F., Molteni, R., Cattaneo, A., Macchi, F., Racagni, G., Gennarelli, M., ... Riva, M. (2010). Long-term duloxetine treatment normalizes altered brain-derived neurotrophic factor expression in serotonin transporter knockout rats through the modulation of specific neurotrophin isoforms [Journal Article]. Molecular Pharmacology, 77(5), 846-853.

Callaway, C. W., \& Geyer, M. A. (1992). Tolerance and cross-tolerance to the activating effects of 3,4-methylenedioxymethamphetamine and a 5-hydroxytryptamine1b agonist [Journal Article]. Journal of Pharmacology and Experimental Therapeutics, 263(1), 318-326.

Callaway, C. W., Johnson, M. P., Gold, L. H., Nichols, D. E., \& Geyer, M. A. (1991). Amphetamine derivatives induce locomotor hyperactivity by acting as indirect serotonin agonists [Journal Article]. Psychopharmacology, 104(3), 293-301.

Callaway, C. W., Rempel, N., Peng, R. Y., \& Geyer, M. A. (1992). Serotonin 5-HT(1)-like receptors mediate hyperactivity in rats induced by 3,4-methylenedioxymethamphetamine [Journal Article]. Neuropsychopharmacology, 7(2), 113-127.

Callaway, C. W., Wing, L. L., \& Geyer, M. A. (1990). Serotonin release contributes to the locomotor stimulant effects of 3,4- 
methylenedioxymethamphetamine in rats [Journal Article]. Journal of Pharmacology and Experimental Therapeutics, 254(2), 456-464.

Cao, J., Hudziak, J. J., \& Li, D. W. (2013). Multi-cultural association of the serotonin transporter gene (SLC6A4) with substance use disorder [Journal Article]. Neuropsychopharmacology, 38(9), 1737-1747.

Cappell, H., \& LeBlanc, A. (1977). Parametric investigations of effects of prior exposure to amphetamine and morphine on conditioned gustatory aversion. Psychopharmacology, 51(3), 265-271.

Caprioli, D., Celentano, M., Dubla, A., Lucantonio, F., Nencini, P., \& Badiani, A. (2009). Ambience and drug choice: Cocaine- and herointaking as a function of environmental context in humans and rats. Biological Psychiatry, 65(10), 893-899.

Caprioli, D., Celentano, M., Paolone, G., \& Badiani, A. (2007). Modeling the role of environment in addiction. Progress In Neuropsychopharmacology \& Biological Psychiatry, 31(8), 1639-1653.

Caprioli, D., Celentano, M., Paolone, G., Lucantonio, F., Bari, A., Nencini, P., \& Badiani, A. (2008). Opposite environmental regulation of heroin and amphetamine self-administration in the rat. Psychopharmacology, 198(3), 395-404.

Carboni, E., Acquas, E., Leone, P., \& Dichiara, G. (1989). 5HT3 receptor antagonists block morphine-induced and nicotine-induced but not amphetamine-induced reward. Psychopharmacology, 97(2), 175-178.

Carelli, R., King, V., Hampson, R., \& Deadwyler, S. (1993). Firing patterns of nucleus-accumbens neurons during cocaine self-administration in rats. Brain Research, 626(1-2), 14-22.

Carlsson, A., Lindqvist, M., Magnusson, T., \& Waldeck, B. (1958). Presence of 3-hydroxytyramine in brain. Science, 127(3296), 471.

Caspi, A., \& Moffitt, T. E. (2006). Opinion - Gene-environment interactions in psychiatry: Joining forces with neuroscience [Journal Article]. Nature Reviews Neuroscience, 7(7), 583-590.

Caspi, A., Sugden, K., Moffitt, T., Taylor, A., Craig, I., Harrington, H., ... 
Poulton, R. (2003). Influence of life stress on depression: Moderation by a polymorphism in the 5-HTT gene [Journal Article]. Science, 301(5631), 386-389.

Cervilla, J., Rivera, M., Molina, E., Torres-Gonzalez, F., Bellon, J., Moreno, B., ... Gutierrez, B. (2006). The 5-HTTLPR s/s genotype at the serotonin transporter gene (SLC6A4) increases the risk for depression in a large cohort of primary care attendees: The PREDICT-gene study. American Journal of Alzheimer's Disease and Other Dementiasf Medical Genetics Part B-neuropsychiatric Genetics, 141B(8), 912-917.

Chang, J., Zhang, L., Janak, P., \& Woodward, D. (1997). Neuronal responses in prefrontal cortex and nucleus accumbens during heroin self-administration in freely moving rats. Brain Research, 754(1-2), 12-20.

Chiao, J. Y., \& Blizinsky, K. D. (2010). Culture - gene coevolution of individualism - collectivism and the serotonin transporter gene [Journal Article]. Proceedings of the Royal Society B: Biological Sciences, 277(1681), 529-537.

Chu, N.-n., Zuo, Y.-f., Meng, L., Lee, D. Y.-W., Han, J.-s., \& Cui, C.-1. (2007). Peripheral electrical stimulation reversed the cell size reduction and increased BDNF level in the ventral tegmental area in chronic morphine-treated rats. Brain Research, 1182, 90-98.

Colado, M. I., O'Shea, E., Granados, R., Murray, T. K., \& Green, A. R. (1997). In vivo evidence for free radical involvement in the degeneration of rat brain 5-HT following administration of mdma ('ecstasy') and p-chloroamphetamine but not the degeneration following fenfluramine [Journal Article]. British Journal of Pharmacology, 121(5), 889-900.

Conner, J. M., Lauterborn, J. C., Yan, Q., Gall, C. M., \& Varon, S. (1997). Distribution of brain-derived neurotrophic factor (BDNF) protein and mRNA in the normal adult rat CNS: Evidence for anterograde axonal transport [Journal Article]. The Journal of Neuroscience, 17(7), 
2295-2313.

Corwin, R., Woolverton, W., Schuster, C., \& Johanson, C. (1987). Anorectics - Effects on food-intake and self-administration in rhesusmonkeys. Alcohol And Drug Research, 7(5-6), 351-361.

Crow, T. J. (1972). Catecholamine-containing neurons and electrical selfstimulation .1. Review of some data [Journal Article]. Psychological Medicine, 2(4), 414-\&.

Dai, S., Corrigall, W. A., Coen, K. M., \& Kalant, H. (1989). Heroin selfadministration by rats - Influence of dose and physical-dependence [Journal Article]. Pharmacology Biochemistry and Behavior, 32(4), 10091015.

Daniela, E., Brennan, K., Gittings, D., Hely, L., \& Schenk, S. (2004). Effect of SCH 23390 on (+/-)-3,4-methylenedioxymethamphetamine hyperactivity and self-administration in rats. Pharmacology Biochemistry and Behavior, 77(4), 745-750.

Degenhardt, L., Whiteford, H., Ferrari, A., Baxter, A., Charlson, F., Hall, W., ... Vos, T. (2013). Global burden of disease attributable to illicit drug use and dependence: Findings from the Global Burden of Disease Study 2010. Lancet, 382(9904), 1564-1574.

Delbruck, S. J. W., Wendel, B., Grunewald, I., Sander, T., MorrisRosendahl, D., Crocq, M. A., ... Hoehe, M. R. (1997). A novel allelic variant of the human serotonin transporter gene regulatory polymorphism [Journal Article]. Cytogenetics and Cell Genetics, 79(34), 214-220.

Deroche-Gamonet, V., Belin, D., \& Piazza, P. V. (2004). Evidence for addiction-like behavior in the rat [Journal Article]. Science, 305(5686), 1014-1017.

Deroche-Gamonet, V., \& Piazza, P. (2010). Transition to addiction [Journal Article]. Encyclopedia of Behavioral Neuroscience, 422-428.

De Vries, T. J., \& Shippenberg, T. S. (2002). Neural systems underlying opiate addiction [Journal Article]. Journal of Neuroscience, 22(9), 3321- 
3325.

Dhawan, B., Cesselin, F., Raghubir, R., Reisine, T., Bradley, P., Portoghese, P., \& Hamon, M. (1996). International union of pharmacology. XII. Classification of opioid receptors [Journal Article]. Pharmacological Reviews, 48(4), 567-592.

Di Matteo, V., De Blasi, A., Di Giulio, C., \& Esposito, E. (2001). Role of $5 \mathrm{HT} 2 \mathrm{C}$ receptors in the control of central dopamine function [Journal Article]. Trends in Pharmacological Sciences, 22(5), 229-232.

Doherty, J. M., \& Frantz, K. J. (2012). Heroin self-administration and reinstatement of heroin-seeking in adolescent vs. adult male rats [Journal Article]. Psychopharmacology, 219(3), 763-773.

Doherty, J. M., \& Frantz, K. J. (2013). Attenuated effects of experimenteradministered heroin in adolescent vs. adult male rats: physical withdrawal and locomotor sensitization [Journal Article]. Psychopharmacology, 225(3), 595-604.

Dulawa, S., Grandy, D., Low, M., Paulus, M., \& Geyer, M. (1999). Dopamine D4 receptor-knock-out mice exhibit reduced exploration of novel stimuli. Journal of Neuroscience, 19(21), 9550-9556.

Duman, R., \& Monteggia, L. (2006). A neurotrophic model for stressrelated mood disorders [Journal Article]. Biological Psychiatry, 59, 1116 - 1127.

Easton, N., \& Marsden, C. A. (2006). Ecstasy: Are animal data consistent between species and can they translate to humans? [Journal Article]. Journal of Psychopharmacology, 20(2), 194-210.

Enkel, T., Gholizadeh, D., von Bohlen Und Halbach, O., Sanchis-Segura, C., Hurlemann, R., Spanagel, R., . . . Vollmayr, B. (2010). Ambiguouscue interpretation is biased under stress- and depression-like states in rats. Neuropsychopharmacology, 35, 10081015.

Ersche, K., Clark, L., London, M., Robbins, T., \& Sahakian, B. (2006). Profile of executive and memory function associated with amphetamine and opiate dependence. Neuropsychopharmacology, 31(5), 1036-1047. 
Ersche, K., Fletcher, P., Lewis, S., Clark, L., Stocks-Gee, G., London, M., ... Sahakian, B. (2005). Abnormal frontal activations related to decisionmaking in current and former amphetamine and opiate dependent individuals. Psychopharmacology, 180(4), 612-623.

Ettenberg, A. (2004). Opponent process properties of self-administered cocaine. Neuroscience And Biobehavioral Reviews, 27(8), 721-728.

Ettenberg, A., \& Geist, T. (1993). Qualitative and quantitative differences in the operant runway behavior of rats working for cocaine and heroin reinforcement. Pharmacology Biochemistry And Behavior, 44(1), 191-198.

Ettenberg, A., Raven, M., Danluck, D., \& Necessary, B. (1999). Evidence for opponent-process actions of intravenous cocaine. Pharmacology Biochemistry And Behavior, 64(3), 507-512.

Evans, K., \& Vaccarino, F. (1990). Amphetamine-induced and morphineinduced feeding - Evidence for involvement of reward mechanisms. Neuroscience And Biobehavioral Reviews, 14(1), 9-22.

Evans, S., \& Johanson, C. (1987). Amphetamine-like effects of anorectics and related-compounds in pigeons. Journal Of Pharmacology And Experimental Therapeutics, 241(3), 817-825.

Everitt, B. J. (2014). Neural and psychological mechanisms underlying compulsive drug seeking habits and drug memories - Indications for novel treatments of addiction [Journal Article]. European Journal of Neuroscience, 40(1), 2163-2182.

Everitt, B. J., Belin, D., Economidou, D., Pelloux, Y., Dalley, J. W., \& Robbins, T. W. (2008). Neural mechanisms underlying the vulnerability to develop compulsive drug-seeking habits and addiction [Journal Article]. Philosophical Transactions of the Royal Society B-Biological Sciences, 363(1507), 3125-3135.

Fabre, V., Beaufour, C., Evrard, A., Rioux, A., Hanoun, N., Lesch, K., ... Martres, M. (2000). Altered expression and functions of serotonin 5-HT1A and 5-HT1B receptors in knock-out mice lacking the 5-HT 
transporter. European Journal of Neuroscience, 12(7), 2299-2310.

Fenu, S., Cadoni, C., \& Di Chiara, G. (2010). Conditioned saccharin avoidance and sensitization to drugs of abuse [Journal Article]. Behavioural Brain Research, 214(2), 248-253.

Fischer, C., Hatzidimitriou, G., Wlos, J., Katz, J., \& Ricaurte, G. (1995). Reorganization of ascending 5-HT axon projections in animals previously exposed to the recreational drug (+/-)3,4methylenedioxymethamphetamine (MDMA, ecstasy) [Journal Article]. Journal of Neuroscience, 15(8), 5476-5485.

Fox, M. A., Andrews, A. M., Wendland, J. R., Lesch, K. P., Holmes, A., \& Murphy, D. L. (2007). A pharmacological analysis of mice with a targeted disruption of the serotonin transporter [Journal Article]. Psychopharmacology, 195(2), 147-166.

Fray, P., Sahakian, B., Robbins, T., Koob, G., \& Iversen, S. (1980). An observational method for quantifying the behavioral-effects of dopamine agonists - Contrasting effects of d-amphetamine and apomorphine. Psychopharmacology, 69(3), 253-259.

Freeman, W. M., Walker, S. J., \& Vrana, K. E. (1999). Quantitative RT-PCR: Pitfalls and potential [Journal Article]. Biotechniques, 26(1), 112-125.

Gaspar, P., \& Lillesaar, C. (2012). Probing the diversity of serotonin neurons [Journal Article]. Philosophical Transactions of the Royal Society B: Biological Sciences, 367(1601), 2382-2394.

Geist, T., \& Ettenberg, A. (1997). Concurrent positive and negative goalbox events produce runway behaviors comparable to those of cocainereinforced rats. Pharmacology Biochemistry And Behavior, 57(1-2), 145150.

Gelernter, J., Kranzler, H., \& Cubells, J. F. (1997). Serotonin transporter protein (SLC6A4) allele and haplotype frequencies and linkage disequilibria in African- and European-American and Japanese populations and in alcohol-dependent subjects [Journal Article]. Human Genetics, 101(2), 243-246. 
Gerra, G., Garofano, L., Santoro, G., Bosari, S., Pellegrini, C., Zaimovic, A., ... Donnini, C. (2004). Association between low-activity serotonin transporter genotype and heroin dependence: Behavioral and personality correlates [Journal Article]. American Journal of Medical Genetics Part B: Neuropsychiatric Genetics, 126B(1), 37-42.

Gillman, P. (1999). The serotonin syndrome and its treatment. Journal of Psychopharmacology, 13(1), 100-109.

Gorwood, P., Batel, P., Ades, J., Hamon, M., \& Boni, C. (2000). Serotonin transporter gene polymorphisms, alcoholism, and suicidal behavior [Journal Article]. Biological Psychiatry, 48(4), 259-264.

Goudie, A. J. (1979). Aversive stimulus properties of drugs [Journal Article]. Neuropharmacology, 18(12), 971-979.

Graham, D. L., Edwards, S., Bachtell, R. K., DiLeone, R. J., Rios, M., \& Self, D. W. (2007). Dynamic BDNF activity in nucleus accumbens with cocaine use increases self-administration and relapse [Journal Article]. Nat Neuroscience, 10(8), 1029-1037.

Grant, B., Stinson, F., Dawson, D., Chou, S., Dufour, M., Compton, W., ... Kaplan, K. (2004). Prevalence and co-occurrence of substance use disorders and independent mood and anxiety disorders - Results from the national epidemiologic survey on alcohol and related conditions. Archives Of General Psychiatry, 61(8), 807-816.

Greco, P., \& Garris, P. (2003). In vivo interaction of cocaine with the dopamine transporter as measured by voltammetry. European Journal Of Pharmacology, 479(1-3), 117-125.

Green, A. R., Mechan, A. O., Elliott, J. M., O'Shea, E., \& Colado, M. I. (2003). The pharmacology and clinical pharmacology of 3,4methylenedioxymethamphetamine (MDMA, "ecstasy") [Journal Article]. Pharmacological Reviews, 55(3), 463-508.

Griffiths, R., Winger, G., Brady, J., \& Snell, J. (1976). Comparison of behavior maintained by infusions of 8 phenylethylamines in baboons. Psychopharmacology, 50(3), 251-258. 
Grigson, P. S. (1997). Conditioned taste aversions and drugs of abuse: A reinterpretation [Journal Article]. Behavioral Neuroscience, 111(1), 129-136.

Grigson, P. S., Lyuboslavsky, P. N., Tanase, D., \& Wheeler, R. A. (1999). Water-deprivation prevents morphine-, but not LiCl-induced, suppression of sucrose intake [Journal Article]. Physiology \& Behavior, 67(2), 277-286.

Grimm, J., Lu, L., Hayashi, T., Hope, B., Su, T., \& Shaham, Y. (2003). Time-dependent increases in brain-derived neurotrophic factor protein levels within the mesolimbic dopamine system after withdrawal from cocaine: Implications for incubation of cocaine craving. Journal of Neuroscience, 23(3), 742-747.

Gudelsky, G., Yamamoto, B., \& Nash, J. (1994). Potentiation of 3,4methylenedioxymethamphetamine-induced dopamine release and serotonin neurotoxicity by 5-HT2 receptor agonists. European Journal Of Pharmacology, 264(3), 325-330.

Gysling, K., \& Wang, R. (1983). Morphine-induced activation of A10 dopamine neurons in the rat. Brain Research, 277(1), 119-127.

Haber, S. N., \& Knutson, B. (2010). The reward circuit: Linking primate anatomy and human imaging [Journal Article]. Neuropsychopharmacology, 35(1), 4-26.

Hagino, Y., Takamatsu, Y., Yamamoto, H., Iwamura, T., Murphy, D. L., Uhl, G. R., ... Ikeda, K. (2011). Effects of mdma on extracellular dopamine and serotonin levels in mice lacking dopamine and/or serotonin transporters [Journal Article]. Current Neuropharmacology, 9(1), 91-95.

Han, D., \& Gu, H. (2006). Comparison of the monoamine transporters from human and mouse in their sensitivities to psychostimulant drugs [Journal Article]. BMC Pharmacology, 6(1), 6.

Hariri, A. R., Mattay, V. S., Tessitore, A., Kolachana, B., Fera, F., Goldman, D., ... Weinberger, D. R. (2002). Serotonin transporter genetic 
variation and the response of the human amygdala [Journal Article]. Science, 297(5580), 400-403.

Harris, J., \& Baldessa.rj. (1973). Uptake of [3H]-catecholamines by homogenates of rat corpus striatum and cerebral-cortex - effects of amphetamine analogs. Neuropharmacology, 12(7), 669-679.

Hayes, D. J., \& Greenshaw, A. J. (2011). 5-HT receptors and reward-related behaviour: A review [Journal Article]. Neuroscience Eamp; Biobehavioral Reviews, 35(6), 1419-1449.

Heffner, T. G., Hartman, J. A., \& Seiden, L. S. (1980). A rapid method for the regional dissection of the rat brain [Journal Article]. Pharmacology Biochemistry and Behavior, 13(3), 453-456.

Heils, A., Teufel, A., Petri, S., Stober, G., Riederer, P., Bengel, D., \& Lesch, K. P. (1996). Allelic variation of human serotonin transporter gene expression [Journal Article]. Journal of Neurochemistry, 66(6), 26212624.

Heinz, A., Braus, D. F., Smolka, M. N., Wrase, J., Puls, I., Hermann, D., ... Buchel, C. (2005). Amygdala-prefrontal coupling depends on a genetic variation of the serotonin transporter [Journal Article]. Nature Neuroscience, 8(1), 20-21.

Homberg, J. R., Boer, S., Raas, H., Olivier, J. A., Verheul, M., Ronken, E., ... Cuppen, E. (2008). Adaptations in pre- and postsynaptic 5-HT1A receptor function and cocaine supersensitivity in serotonin transporter knockout rats [Journal Article]. Psychopharmacology, 200(3), 367-380.

Homberg, J. R., Olivier, J. D. A., Smits, B. M. G., Mul, J. D., Mudde, J., Verheul, M., ... Cuppen, E. (2007). Characterization of the serotonin transporter knockout rat: A selective change in the functioning of the serotonergic system [Journal Article]. Neuroscience, 146(4), 16621676.

Hornung, J.-P. (2003). The human raphe nuclei and the serotonergic system [Journal Article]. Journal of Chemical Neuroanatomy, 26(4), 331343. 
Huang, C.-C., Lin, H.-J., \& Hsu, K.-S. (2007). Repeated cocaine administration promotes long-term potentiation induction in rat medial prefrontal cortex. Cerebral Cortex, 17(8), 1877-1888.

Hughes, P. H., Barker, N. W., Crawford, G. A., \& Jaffe, J. H. (1972). The natural history of a heroin epidemic [Journal Article]. American Journal of Public Health, 62(7), 995-1001.

Hunt, T., \& Amit, Z. (1987). Conditioned taste-aversion induced by self-administered drugs - Paradox revisited [Journal Article]. Neuroscience and Biobehavioral Reviews, 11(1), 107-130.

Igncio, Z. M., Rus, G. Z., Abelaira, H. M., \& Quevedo, J. (2014). Epigenetic and epistatic interactions between serotonin transporter and brain-derived neurotrophic factor genetic polymorphism: Insights in depression [Journal Article]. Neuroscience, 275(0), 455-468.

Isaacson, R., Yongue, B., \& Mcclearn, D. (1978). Dopamine agonists - Their effect on locomotion and exploration. Behavioral Biology, 23(2), 163179.

Iversen, S. D., \& Iversen, L. L. (2007). Dopamine: 50 years in perspective [Journal Article]. Trends in Neurosciences, 30(5), 188-193.

Jacobs, E. H., Spijker, S., Verhoog, C. W., Kamprath, K., de Vries, T. J., Smit, A. B., \& Schoffelmeer, A. N. M. (2002). Active heroin administration induces specific genomic responses in the nucleus accumbens shell [Journal Article]. Faseb Journal, 16(12), 1961-1963.

Johnson, S. W., \& North, R. A. (1992). Opioids excite dopamine neurons by hyperpolarization of local interneurons [Journal Article]. Journal of Neuroscience, 12(2), 483-488.

Justice, J. B., Michael, A. C., \& Neill, D. B. (1985). In vivo voltammetry. In Amines and their metabolites (pp. 197-266). Springer.

Kalivas, P., \& Duffy, P. (1987). Sensitization to repeated morphine injection in the rat - Possible involvement of A10 dopamine neurons. Journal of Pharmacology and Experimental Therapeutics, 241(1), 204-212.

Kalueff, A. V., LaPorte, J. L., \& Murphy, D. L. (2008). Perspectives on 
genetic animal models of serotonin toxicity. Neurochemistry International, 52(4-5), 649-658.

Karg, K., Burmeister, M., Shedden, K., \& Sen, S. (2011). The serotonin transporter promoter variant (5-HTTLPR), stress, and depression meta-analysis revisited: Evidence of genetic moderation [Journal Article]. Archives of General Psychiatry, 68(5), 444-454.

Kasanetz, F., Deroche-Gamonet, V., Berson, N., Balado, E., Lafourcade, M., Manzoni, O., \& Piazza, P. V. (2010). Transition to addiction is associated with a persistent impairment in synaptic plasticity [Journal Article]. Science, 328(5986), 1709-1712.

Kendall, J. M., \& Latter, V. S. (2003). Intranasal diamorphine as an alternative to intramuscular morphine - Pharmacokinetic and pharmacodynamic aspects [Journal Article]. Clinical Pharmacokinetics, 42(6), 501-513.

Kenna, G., Roder-Hanna, N., Leggio, L., Zywiak, W., Clifford, J., Edwards, S., ... Swift, R. (2012). Association of the 5-HTT gene-linked promoter region (5-HTTLPR) polymorphism with psychiatric disorders: Review of psychopathology and pharmacotherapy [Journal Article]. Pharmacogenomics and Personalized Medicine, 5(1), 19-35.

Kilpatrick, D., Ruggiero, K., Acierno, R., Saunders, B., Resnick, H., \& Best, C. (2003). Violence and risk of PTSD, major depression, substance abuse/dependence, and comorbidity: Results from the national survey of adolescents [Article]. Journal of Consulting and Clinical Psychology, 71(4), 692-700.

Kirby, L. G., Zeeb, F. D., \& Winstanley, C. A. (2011). Contributions of serotonin in addiction vulnerability [Journal Article]. Neuropharmacology, 61(3), 421-432.

Kish, S. J., Kalasinsky, K. S., Derkach, P., Schmunk, G. A., Guttman, M., Ang, L., ... Haycock, J. W. (2001). Striatal dopaminergic and serotonergic markers in human heroin users [Journal Article]. Neuropsychopharmacology, 24(5), 561-7. 
Koob, G. F. (1992). Drugs of abuse - Anatomy, pharmacology and function of reward pathways [Journal Article]. Trends in Pharmacological Sciences, 13(5), 177-184.

Koob, G. F., Lloyd, G., \& Mason, B. (2009). Development of pharmacotherapies for drug addiction: A rosetta stone approach [Journal Article]. Nature Reviews Drug Discovery, 8(6), 500-515.

Koob, G. F., Sanna, P., \& Bloom, F. (1998). Neuroscience of addiction [Journal Article]. Neuron, 21(3), 467-476.

Koob, G. F., \& Volkow, N. D. (2010). Neurocircuitry of addiction [Journal Article]. Neuropsychopharmacology, 35(4), 1051-1051.

Kotler, M., Cohen, H., Kremer, I., Mel, H., Horowitz, R., Ohel, N., ... Ebstein, R. P. (1999). No association between the serotonin transporter promoter region (5-HTTLPR) and the dopamine $\mathrm{d} 3$ receptor (Ball D3DR) polymorphisms and heroin addiction [Journal Article]. Molecular Psychiatry, 4(4), 313.

Kozisek, M. E., Middlemas, D., \& Bylund, D. B. (2008). Brain-derived neurotrophic factor and its receptor tropomyosin-related kinase $\mathrm{b}$ in the mechanism of action of antidepressant therapies [Journal Article]. Pharmacology \& Therapeutics, 117(1), 30-51.

Kranz, G. S., Kasper, S., \& Lanzenberger, R. (2010). Reward and the serotonergic system [Journal Article]. Neuroscience, 166(4), 1023-1035.

Kreek, M. J., Levran, O., Reed, B., Schlussman, S. D., Zhou, Y., \& Butelman, E. R. (2012). Opiate addiction and cocaine addiction: Underlying molecular neurobiology and genetics [Journal Article]. Journal of Clinical Investigation, 122(10), 3387-3393.

Kruk, Z., \& Pycock, C. (1993a). 5-hydroxytryptamine. Neurotransmitters and drugs, 3rd edition. Used with permission.

Kruk, Z., \& Pycock, C. (1993b). Dopamine. Neurotransmitters and drugs, 3rd edition. Used with permission.

Kuntz-Melcavage, K. L., Brucklacher, R. M., Grigson, P. S., Freeman, W. M., \& Vrana, K. E. (2009). Gene expression changes following 
extinction testing in a heroin behavioral incubation model. BMC Neuroscience, 10.

Kunugi, H., Hattori, M., Kato, T., Tatsumi, M., Sakai, T., Sasaki, T., ... Nanko, S. (1997). Serotonin transporter gene polymorphisms: Ethnic difference and possible association with bipolar affective disorder [Journal Article]. Molecular Psychiatry, 2(6), 457-462.

Laucht, M., Treutlein, J., Schmid, B., Blomeyer, D., Becker, K., Buchmann, A. F., ... Banaschewski, T. (2009). Impact of psychosocial adversity on alcohol intake in young adults: Moderation by the 11 genotype of the serotonin transporter polymorphism [Journal Article]. Biological Psychiatry, 66(2), 102-109.

Le Foll, B., Diaz, J., \& Sokoloff, P. (2005). A single cocaine exposure increases BDNF and D-3 receptor expression: implications for drugconditioning. Neuroreport, 16(2), 175-178.

Lenoir, M., Guillem, K., Koob, G. F., \& Ahmed, S. H. (2012). Drug specificity in extended access cocaine and heroin self-administration. Addiction Biology, 17(6), 964-976.

Lesch, K. P., Bengel, D., Heils, A., Sabol, S. Z., Greenberg, B. D., Petri, S., ... Murphy, D. L. (1996). Association of anxiety-related traits with a polymorphism in the serotonin transporter gene regulatory region [Journal Article]. Science, 274(5292), 1527-1531.

Lesch, K. P., \& Gutknecht, L. (2005). Pharmacogenetics of the serotonin transporter [Journal Article]. Progress in Neuro-Psychopharmacology and Biological Psychiatry, 29(6), 1062-1073.

Li, T., Liu, X., Zhao, J., Hu, X., Ball, D., Loh, E., .. Collier, D. (2002). Allelic association analysis of the dopamine D2, D3, 5-HT2A, and GABA(A)gamma 2 receptors and serotonin transporter genes with heroin abuse in Chinese subjects. American Journal Of Medical Genetics, 114(3), 329-335.

Li, X., DeJoseph, M. R., Urban, J. H., Bahi, A., Dreyer, J.-L., Meredith, G. E., ... Wolf, M. E. (2013). Different roles of BDNF in nucleus accum- 
bens core versus shell during the incubation of cue-induced cocaine craving and its long-term maintenance. Journal of Neuroscience, 33(3), 1130-1142.

Li, X., Rubio, F. J., Zeric, T., Bossert, J. M., Kambhampati, S., Cates, H. M., ... Shaham, Y. (2015). Incubation of methamphetamine craving is associated with selective increases in expression of BDNF and Trkb, glutamate receptors, and epigenetic enzymes in cue-activated fosexpressing dorsal striatal neurons. Journal of Neuroscience, 35(21), 8232-8244.

Lin, J. Y., Arthurs, J., \& Reilly, S. (2014). Conditioned taste aversion, drugs of abuse and palatability [Journal Article]. Neuroscience and Biobehavioral Reviews, 45, 28-45.

Lira, A., Zhou, M. M., Castanon, N., Ansorge, M. S., Gordon, J. A., Francis, J. H., ... Gingrich, J. A. (2003). Altered depression-related behaviors and functional changes in the dorsal raphe nucleus of serotonin transporter-deficient mice [Journal Article]. Biological Psychiatry, 54(10), 960-971.

Liu, Q.-R., Lu, L., Zhu, X.-G., Gong, J.-P., Shaham, Y., \& Uhl, G. R. (2006). Rodent BDNF genes, novel promoters, novel splice variants, and regulation by cocaine [Journal Article]. Brain Research, 1067(1), 1-12.

Lizarraga, L. E., Phan, A. V., Cholanians, A. B., Herndon, J. M., Lau, S. S., \& Monks, T. J. (2014). Serotonin reuptake transporter deficiency modulates the acute thermoregulatory and locomotor activity response to 3,4-()-methylenedioxymethamphetamine, and attenuates depletions in serotonin levels in SERT-KO rats [Journal Article]. Toxicological Sciences.

Louria, D. B., Hensle, T., \& Rose, J. (1967). The major medical complications of heroin addiction [Journal Article]. Annals of Internal Medicine, 67(1), 1-22.

Lu, H., Cheng, P.-1., Lim, B. K., Khoshnevisrad, N., \& Poo, M.-m. (2010). Elevated BDNF after cocaine withdrawal facilitates LTP in medial 
prefrontal cortex by suppressing GABA inhibition. Neuron, 67(5), 821-833.

Lyon, M., \& Robbins, T. (1975). The action of central nervous system stimulant drugs a general theory concerning amphetamine effects (W. Essman \& L. Valzelli, Eds.). New York: Spectrum.

Malberg, J. E., \& Seiden, L. S. (1998). Small changes in ambient temperature cause large changes in 3,4-methylenedioxymethamphetamine (MDMA)-induced serotonin neurotoxicity and core body temperature in the rat [Journal Article]. Journal of Neuroscience, 18(13), 50865094.

Mann, J. J. (1999). Role of the serotonergic system in the pathogenesis of major depression and suicidal behavior [Journal Article]. Neuropsychopharmacology, 21(2), S99-S105.

Martinez-Turrillas, R., Moyano, S., Del Rio, J., \& Frechilla, D. (2006). Differential effects of 3,4-methylenedioxymethamphetamine (MDMA, "ecstasy") on BDNF mRNA expression in rat frontal cortex and hippocampus. Neuroscience Letters, 402(1-2), 126-130.

McCann, U. D., \& Ricaurte, G. A. (1993). Reinforcing subjective effects of (+/-) 3,4-methylenedioxymethamphetamine (ecstasy) may be separable from its neurotoxic actions - Clinical-evidence [Journal Article]. Journal of Clinical Psychopharmacology, 13(3), 214-217.

McCann, U. D., Szabo, Z., Scheffel, U., Dannals, R. F., \& Ricaurte, G. A. (1998). Positron emission tomographic evidence of toxic effect of mdma ("ecstasy") on brain serotonin neurons in human beings [Journal Article]. Lancet, 352(9138), 1433-1437.

McDowell, D. M., \& Kleber, H. D. (1994). MDMA - Its history and pharmacology [Journal Article]. Psychiatric Annals, 24(3), 127-130.

McFarland, K., \& Kalivas, P. (2001). The circuitry mediating cocaineinduced reinstatement of drug-seeking behavior. Journal Of Neuroscience, 21(21), 8655-8663.

McGinty, J. F., Whitfield, T. W., \& Berglind, W. J. (2010). Brain-derived 
neurotrophic factor and cocaine addiction [Journal Article]. Brain Research, 1314(0), 183-193.

McGonigle, P., \& Ruggeri, B. (2014). Animal models of human disease: Challenges in enabling translation [Journal Article]. Biochemical Pharmacology, 87(1), 162-171.

McNamara, R., Dalley, J. W., Robbins, T. W., Everitt, B. J., \& Belin, D. (2010). Trait-like impulsivity does not predict escalation of heroin self-administration in the rat. Psychopharmacology, 212(4), 453-464.

Merenakk, L., Maestu, J., Nordquist, N., Parik, J., Oreland, L., Loit, H. M., \& Harro, J. (2011). Effects of the serotonin transporter (5-HTTLPR) and alpha(2A)-adrenoceptor (C-1291G) genotypes on substance use in children and adolescents: a longitudinal study [Journal Article]. Psychopharmacology, 215(1), 13-22.

Meyer, Q. L., J.S. (2005). Psychopharmacology: Drugs, the brain and behavior [Book]. Sunderland, Massachusetts USA: Sinauer Associates, Inc.

Michaelovsky, E., Frisch, A., Rockah, R., Peleg, L., Magal, N., Shohat, M., \& Weizman, R. (1999). A novel allele in the promoter region of the human serotonin transporter gene [Journal Article]. Molecular Psychiatry, 4(1), 97-99.

Milton, A. L., \& Everitt, B. J. (2012). The persistence of maladaptive memory: Addiction, drug memories and anti-relapse treatments [Journal Article]. Neuroscience Eamp; Biobehavioral Reviews, 36(4), 1119-1139.

Molteni, R., Calabrese, F., Cattaneo, A., Mancini, M., Gennarelli, M., Racagni, G., \& Riva, M. (2009). Acute stress responsiveness of the neurotrophin BDNF in the rat hippocampus is modulated by chronic treatment with the antidepressant duloxetine [Journal Article]. Neuropsychopharmacol, 34, 1523 - 32.

Molteni, R., Cattaneo, A., Calabrese, F., Macchi, F., Olivier, J. D. A., Racagni, G., ... Riva, M. A. (2010). Reduced function of the serotonin transporter is associated with decreased expression of BDNF in rodents as well as in humans [Journal Article]. Neurobiology of Dis- 
ease, 37(3), 747-755.

Mortensen, O. V., Thomassen, M., Larsen, M. B., Whittemore, S. R., \& Wiborg, O. (1999). Functional analysis of a novel human serotonin transporter gene promoter in immortalized raphe cells [Journal Article]. Molecular Brain Research, 68(1-2), 141-148.

Mosner, A., Kuhlman, G., Roehm, C., \& Vogel, W. (1997). Serotonergic receptors modify the voluntary intake of alcohol and morphine but not of cocaine and nicotine by rats. Pharmacology, 54(4), 186-192.

Mueller, C. P., \& Homberg, J. R. (2015). The role of serotonin in drug use and addiction. Behavioural Brain Research, 277(SI), 146-192.

Murphy, D. L., Li, Q., Engel, S., Wichems, C., Andrews, A., Lesch, K.-P., \& Uhl, G. (2001). Genetic perspectives on the serotonin transporter [Journal Article]. Brain Research Bulletin, 56(5), 487-494.

Musto, D. F. (1991). Opium, cocaine and marijuana in American history [Journal Article]. Scientific American, 265(1), 40-47.

Nakamura, M., Ueno, S., Sano, A., \& Tanabe, H. (2000). The human serotonin transporter gene linked polymorphism (5-HTTLPR) shows ten novel allelic variants [Journal Article]. Molecular Psychiatry, 5(1), 32.

Nestler, E. J. (1996). Under siege: The brain on opiates [Journal Article]. Neuron, 16(5), 897-900.

Nestler, E. J. (2001). Molecular neurobiology of addiction [Journal Article]. The American Journal on Addictions, 10(3), 201-217.

Nestler, E. J. (2004). Historical review: Molecular and cellular mechanisms of opiate and cocaine addiction [Journal Article]. Trends in Pharmacological Sciences, 25(4), 210-218.

Nolan, T., Hands, R. E., \& Bustin, S. A. (2006). Quantification of mRNA using real-time RT-PCR [Journal Article]. Nature Protocols, 1(3), 15591582.

Nonkes, L. J. P., Van Bussel, I. P. G., Verheij, M. M. M., \& Homberg, J. R. (2011). The interplay between brain 5-hydroxytryptamine levels and cocaine addiction [Journal Article]. Behavioural Pharmacology, 22(8), 
723-738.

Noskova, T., Pivac, N., Nedic, G., Kazantseva, A., Gaysina, D., Faskhutdinova, G., ... Seler, D. M. (2008). Ethnic differences in the serotonin transporter polymorphism (5-httlpr) in several European populations [Journal Article]. Progress in Neuro-Psychopharmacology and Biological Psychiatry, 32(7), 1735-1739.

Oakly, A. C., Brox, B. W., Schenk, S., \& Ellenbroek, B. A. (2014). A genetic deletion of the serotonin transporter greatly enhances the reinforcing properties of MDMA in rats [Journal Article]. Molecular Psychiatry, 19(5), 534-535.

Ohearn, E., Battaglia, G., Desouza, E. B., Kuhar, M. J., \& Molliver, M. E. (1988). Methylenedioxyamphetamine (MDA) and methylenedioxymethamphetamine (MDMA) cause selective ablation of serotonergic axon terminals in forebrain - Immunocytochemical evidence for neurotoxicity [Journal Article]. Journal of Neuroscience, 8(8), 2788-2803.

Oldendor, W. H., Oldendor, S. Z., Braun, L., \& Hyman, S. (1972). Blood-brain barrier - Penetration of morphine, codeine, heroin, and methadone after carotid injection [Journal Article]. Science, 178(4064), 984-\&.

Olds, J., \& Milner, P. (1954). Positive reinforcement produced by electrical stimulation of septal area and other regions of rat brain [Journal Article]. Journal of Comparative and Physiological Psychology, 47(6), 419427.

Olivier, J. D. A., Van Der Hart, M. G. C., Van Swelm, R. P. L., Dederen, P. J., Homberg, J. R., Cremers, T., ... Ellenbroek, B. A. (2008). A study in male and female 5-HT transporter knockout rats: An animal model for anxiety and depression disorders [Journal Article]. Neuroscience, 152(3), 573-584.

Panlilio, L. V., \& Goldberg, S. R. (2007). Self-administration of drugs in animals and humans as a model and an investigative tool [Journal 
Article]. Addiction, 102(12), 1863-1870.

Papciak, J., Popik, P., Fuchs, E., \& Rygula, R. (2013). Chronic psychosocial stress makes rats more pessimistic in the ambiguous-cue interpretation paradigm. Behavioural Brain Research, 256, 305 - 310.

Pardridge, W. M. (2012). Drug transport across the blood-brain barrier [Journal Article]. Journal of Cerebral Blood Flow E Metabolism, 32(11), 1959-1972.

Parker, L. A. (1995). Rewarding drugs produce taste avoidance, but not taste-aversion [Journal Article]. Neuroscience and Biobehavioral Reviews, 19(1), 143-151.

Parrott, A. (2002). Recreational ecstasy/MDMA, the serotonin syndrome, and serotonergic neurotoxicity [Journal Article]. Pharmacology Biochemistry and Behavior, 71(4), 837-844.

Pelloux, Y., Dilleen, R., Economidou, D., Theobald, D., \& Everitt, B. J. (2012). Reduced forebrain serotonin transmission is causally involved in the development of compulsive cocaine seeking in rats [Journal Article]. Neuropsychopharmacology, 37(11), 2505-2514.

Pettit, H. O., \& Justice, J. B. (1989). Dopamine in the nucleus accumbens during cocaine self-administration as studied by invivo microdialysis [Journal Article]. Pharmacology Biochemistry and Behavior, 34(4), 899-904.

Pickens, R., \& Harris, W. (1968). Self-administration of d-amphetamine by rats. Psychopharmacologia, 12(2), 158-\&.

Pijnenburg, A. J. J., \& van Rossum, J. M. (1973). Stimulation of locomotor activity following injection of dopamine into the nucleus accumbens [Journal Article]. Journal of Pharmacy and Pharmacology, 25(12), 10031005.

Porras, G., Di Matteo, V., De Deurwaerdere, P., Esposito, E., \& Spampinato, U. (2002). Central serotonin(4) receptors selectively regulate the impulse-dependent exocytosis of dopamine in the rat striatum: in vivo studies with morphine, amphetamine and cocaine. Neurophar- 
macology, 43(7), 1099-1109.

Porras, G., Di Matteo, V., Fracasso, C., Lucas, G., De Deurwaerdere, P., Caccia, S., ... Spampinato, U. (2002). 5-HT2A and 5-HT2C/2B receptor subtypes modulate dopamine release induced in vivo by amphetamine and morphine in both the rat nucleus accumbens and striatum. Neuropsychopharmacology, 26(3), 311-324.

Ranaldi, R., Egan, J., Kest, K., Fein, M., \& Delamater, A. R. (2009). Repeated heroin in rats produces locomotor sensitization and enhances appetitive pavlovian and instrumental learning involving food reward [Journal Article]. Pharmacology Biochemistry and Behavior, 91(3), 351-357.

Rattray, M. (1991). Ecstasy - Towards an understanding of the biochemical basis of the actions of MDMA [Journal Article]. Essays in Biochemistry, 26, 77-87.

Regier, D., Farmer, M., Rae, D., Locke, B., Keith, S., Judd, L., \& Goodwin, F. (1990). Comorbidity of mental disorders with alcohol and other drug abuse: Results from the epidemiologic catchment area (eca) study. Journal of the Americal Medical Association, 264(19), 2511-2518.

Reinhold, J. A., Mandos, L. A., Rickels, K., \& Lohoff, F. W. (2011). Pharmacological treatment of generalized anxiety disorder [Journal Article]. Expert Opinion on Pharmacotherapy, 12(16), 2457-2467.

Ricaurte, G. A., Delanney, L. E., Irwin, I., \& Langston, J. W. (1988). Toxic effects of MDMA on central serotonergic neurons in the primate Importance of route and frequency of drug administration [Journal Article]. Brain Research, 446(1), 165-168.

Ricaurte, G. A., Finnegan, K. T., Irwin, I., \& Langston, J. W. (1990). Aminergic metabolites in cerebrospinal-fluid of humans previously exposed to MDMA - Preliminary observations [Journal Article]. Annals of the New York Academy of Sciences, 600, 699-710.

Ricaurte, G. A., Yuan, J., \& McCann, U. D. (2000). (+/-)3,4methylenedioxymethamphetamine ('ecstasy')-induced serotonin 
neurotoxicity: Studies in animals [Journal Article]. Neuropsychobiology, 42(1), 5-10.

Richardson, N. R., \& Roberts, D. C. S. (1996). Progressive ratio schedules in drug self-administration studies in rats: A method to evaluate reinforcing efficacy [Journal Article]. Journal of Neuroscience Methods, 66(1), 1-11.

Robbins, T. W., \& Everitt, B. J. (1996). Neurobehavioural mechanisms of reward and motivation [Journal Article]. Current Opinion in Neurobiology, 6(2), 228-236.

Robinson, T., \& Berridge, K. (1993). The neural basis of drug craving: An incentive-sensitization theory of addiction [Journal Article]. Brain Research Reviews, 18(3), 247-291.

Robinson, T., \& Berridge, K. (2001). Incentive-sensitization and addiction [Journal Article]. Addiction, 96(1), 103-114.

Robinson, T., \& Kolb, B. (1997). Persistent structural modifications in nucleus accumbens and prefrontal cortex neurons produced by previous experience with amphetamine. Journal Of Neuroscience, 17(21), 8491-8497.

Robinson, T., \& Kolb, B. (2004). Structural plasticity associated with exposure to drugs of abuse. Neuropharmacology, 47(S), 33-46.

Rogers, J. L., Ghee, S., \& See, R. E. (2008). The neural circuitry underlying reinstatement of heroin-seeking behavior in an animal model of relapse. Neuroscience, 151(2), 579-588.

Rook, E. J., Huitema, A. D. R., van den Brink, W., van Ree, J. M., \& Beijnen, J. H. (2006). Population pharmacokinetics of heroin and its major metabolites [Journal Article]. Clinical Pharmacokinetics, 45(4), 401-417.

Rothman, R. B., \& Baumann, M. H. (2003). Monoamine transporters and psychostimulant drugs [Journal Article]. European Journal of Pharmacology, 479(1-3), 23-40.

Rothman, R. B., \& Baumann, M. H. (2006). Balance between dopamine 
and serotonin release modulates behavioral effects of amphetaminetype drugs [Book Section]. In S. F. Ali \& F. Fornai (Eds.), Cellular and molecular mechanisms of drugs of abuse and neurotoxicity: Cocaine, ghb, and substituted amphetamines (Vol. 1074, p. 245-260).

Sadri-Vakili, G., Kumaresan, V., Schmidt, H. D., Famous, K. R., Chawla, P., Vassoler, F. M., ... Cha, J. H. J. (2010). Cocaine-induced chromatin remodeling increases brain-derived neurotrophic factor transcription in the rat medial prefrontal cortex, which alters the reinforcing efficacy of cocaine [Journal Article]. Journal of Neuroscience, 30(35), 11735-11744.

Saiz, P. A., Garcia-Portilla, M. P., Arango, C., Morales, B., MartinezBarrondo, S., Alvarez, C., ... Bobes, J. (2008). Association between heroin dependence and 5-HT2A receptor gene polymorphisms. European Addiction Research, 14(1), 47-52.

Saiz, P. A., Garcia-Portilla, M. P., Florez, G., Arango, C., Corcoran, P., Morales, B., ... Bobes, J. (2009). Differential role of serotonergic polymorphisms in alcohol and heroin dependence. Progress In Neuropsychopharmacology \& Biological Psychiatry, 33(4), 695-700.

Salvatore, M. F., Hoffman, A. F., Burmeister, J. J., \& Gerhardt, G. A. (2003). In vivo voltammetry in drug abuse research (B. Waterhouse, Ed.). CRC Press, Taylor \& Francis Group.

Sanger, D., \& McCarthy, P. (1980). Differential-effects of morphine on food and water-intake in food deprived and freely-feeding rats. Psychopharmacology, 72(1), 103-106.

Sargeant, T. J. (2008). The effect of opiates on the developing cerebral cortex (Unpublished doctoral dissertation). School of Biological Sciences, Victoria University of Wellington.

Schenk, S. (2009). MDMA self-administration in laboratory animals: A summary of the literature and proposal for future research [Journal Article]. Neuropsychobiology, 60(3-4), 130-6.

Schenk, S. (2011). MDMA ("ecstasy") abuse as an example of dopa- 
mine neuroplasticity [Journal Article]. Neuroscience $\mathcal{E}$ Biobehavioral Reviews, 35(5), 1203-1218.

Schenk, S., Colussi-Mas, J., Do, J., \& Bird, J. (2012). Profile of MDMA self-administration from a large cohort of rats: MDMA develops a profile of dependence with extended testing [Journal Article]. Journal of Drug and Alcohol Research, 1, 1-6.

Schmidt, C., Fadayel, G., Sullivan, C., \& Taylor, V. (1992). 5-HT(2)receptors exert a state-dependent regulation of dopaminergic function - studies with MDL-100,907 and the amphetamine analog, 3,4methylenedioxymethamphetamine. European Journal Of Pharmacology, 223(1), 65-74.

Schmidt, H. D., Sangrey, G. R., Darnell, S. B., Schassburger, R. L., Cha, J.H. J., Pierce, R. C., \& Sadri-Vakili, G. (2012). Increased brain-derived neurotrophic factor (BDNF) expression in the ventral tegmental area during cocaine abstinence is associated with increased histone acetylation at BDNF exon I-containing promoters [Journal Article]. Journal of Neurochemistry, 120(2), 202-209.

Schmittgen, T. D., \& Livak, K. J. (2008). Analyzing real-time PCR data by the comparative C-T method [Journal Article]. Nature Protocols, 3(6), 1101-1108.

Semple, D. M., Ebmeier, K. P., Glabus, M. F., O'Carroll, R. E., \& Johnstone, E. C. (1999). Reduced in vivo binding to the serotonin transporter in the cerebral cortex of mdma ('ecstasy') users [Journal Article]. British Journal of Psychiatry, 175, 63-69.

Shanahan, N. A., Pierz, K. A. H., Masten, V. L., Waeber, C., Ansorge, M., Gingrich, J. A., ... Dulawa, S. C. (2009). Chronic reductions in serotonin transporter function prevent 5-HT1B-induced behavioral effects in mice. Biological Psychiatry, 65(5), 401-408.

Sharkey, J., McBean, D. E., \& Kelly, P. A. T. (1991). Alterations in hippocampal function following repeated exposure to the amphetamine derivative methylenedioxymethamphetamine (ecstasy) [Journal Ar- 
ticle]. Psychopharmacology, 105(1), 113-118.

Sklair-Tavron, L., Shi, W., Lane, S., Harris, H., Bunney, B., \& Nestler, E. (1996). Chronic morphine induces visible changes in the morphology of mesolimbic dopamine neurons. Proceedings Of The National Academy Of Sciences Of The United States Of America, 93(20), 1120211207.

Slack, A., Nana, G., Webster, M., Stokes, F., \& Wu, J. (2009). Costs of harmful alcohol and other drug abuse [Journal Article]. Wellington, New Zealand: Business and Economic Research Limited.

Smith, R. J., \& Aston-Jones, G. (2012). OrexinŁ/Łhypocretin 1 receptor antagonist reduces heroin self-administration and cue-induced heroin seeking [Journal Article]. European Journal of Neuroscience, 35(5), 798-804.

Smits, B. M. G., Mudde, J. B., van de Belt, J., Verheul, M., Olivier, J., Homberg, J., ... Cuppen, E. (2006). Generation of gene knockouts and mutant models in the laboratory rat by ENU-driven targetseletted mutagenesis [Journal Article]. Pharmacogenetics and Genomics, 16(3), 159-169.

Sora, I., Hall, F. S., Andrews, A. M., Itokawa, M., Li, X. F., Wei, H. B., ... Uhl, G. R. (2001). Molecular mechanisms of cocaine reward: Combined dopamine and serotonin transporter knockouts eliminate cocaine place preference [Journal Article]. Proceedings of the National Academy of Sciences of the United States of America, 98(9), 5300-5305.

Sora, I., Wichems, C., Takahashi, N., Li, X. F., Zeng, Z. Z., Revay, R., ... Uhl, G. R. (1998). Cocaine reward models: Conditioned place preference can be established in dopamine- and in serotonin-transporter knockout mice [Journal Article]. Proceedings of the National Academy of Sciences of the United States of America, 95(13), 7699-7704.

Spagnolo, P. A., Celentano, M., Dubla, A., \& Badiani, A. (2011). Setting preferences for heroin versus cocaine taking in human co-abusers: Role of environmental variables in drug use and relapse. Behavioural 
Pharmacology, 22(S), E21.

Strang, J., Griffiths, P., \& Gossop, M. (1997). Heroin smoking by 'chasing the dragon': Origins and history [Journal Article]. Addiction, 92(6), 673-684.

Su, S., Zhao, J., Bremner, J. D., Miller, A. H., Tang, W., Bouzyk, M., ... Vaccarino, V. (2009). Serotonin transporter gene, depressive symperspectiveInterleukin-6 / Clinical Perspective [Journal Article]. Circulation: Cardiovascular Genetics, 2(6), 614-620.

Tan, E., Yeo, B., Ho, B., Tay, A., \& Tan, C. (1999). Evidence for an association between heroin dependence and a VNTR polymorphism at the serotonin transporter locus. Molecular Psychiatry, 4(3), 215-217.

Taurines, R., Gerlach, M., Warnke, A., Thome, J., \& Wewetzer, C. (2011). Pharmacotherapy in depressed children and adolescents [Journal Article]. World Journal of Biological Psychiatry, 12, 11-15.

Theberge, F. R., Liu, Q. R., Fanous, S., Pickens, C. L., Goldart, E., Hope, B., \& Shaham, Y. (2011). Incubation of heroin craving is not associated with alterations in BDNF, TrkB, or MeCP2 expression in nucleus accumbens, dorsal striatum, or medial prefrontal cortex (Vol. 41).

Theberge, F. R., Pickens, C. L., Goldart, E., Fanous, S., Hope, B. T., Liu, Q., \& Shaham, Y. (2012). Association of time-dependent changes in $\mathrm{mu}$ opioid receptor mRNA, but not BDNF, TrkB, or MeCP2 mRNA and protein expression in the rat nucleus accumbens with incubation of heroin craving. Psychopharmacology, 224(4), 559-571.

Thomsen, M., Hall, F. S., Uhl, G. R., \& Caine, S. B. (2009). Dramatically decreased cocaine self-administration in dopamine but not serotonin transporter knock-out mice. Journal of Neuroscience, 29(4), 1087-1092.

Tork, I. (1990). Anatomy of the serotonergic systema [Journal Article]. Annals of the New York Academy of Sciences, 600(1), 9-34.

Trigo, J. M., Renoir, T., Lanfumey, L., Hamon, M., Lesch, K. P., Robledo, P., \& Maldonado, R. (2007). 3,4-methylenedioxymethamphetamine selfadministration is abolished in serotonin transporter knockout mice 
[Journal Article]. Biological Psychiatry, 62(6), 669-679.

Ungerstedt, U. (1979). Central dopamine mechanisms and behaviour. (A. Horn, J. Korf, \& B. Westerink, Eds.). Academic Press.

Ungerstedt, U. (1991). Microdialysis - Principles and applications for studies in animals and man. Journal of Internal Medicine, 230(4), 365-373.

United Nations Office on Drugs and Crime. (2014). World Drug Report 2014 [Booklet].

Van den Oever, M. C., Goriounova, N. A., Li, K. W., Van der Schors, R. C., Binnekade, R., Schoffelmeer, A. N. M., ... De Vries, T. J. (2008). Prefrontal cortex AMPA receptor plasticity is crucial for cue-induced relapse to heroin-seeking. Nature Neuroscience, 11(9), 1053-1058.

Vanderschuren, L., \& Kalivas, P. (2000). Alterations in dopaminergic and glutamatergic transmission in the induction and expression of behavioral sensitization: A critical review of preclinical studies. Psychopharmacology, 151(2-3), 99-120.

VanGuilder, H. D., Vrana, K. E., \& Freeman, W. M. (2008). Twenty-five years of quantitative PCR for gene expression analysis [Journal Article]. Biotechniques, 44(5), 619-626.

van Ijzendoorn, M. H., Belsky, J., \& Bakermans-Kranenburg, M. J. (2012). Serotonin transporter genotype 5HTTLPR as a marker of differential susceptibility: A meta-analysis of child and adolescent gene-byenvironment studies [Journal Article]. Transl Psychiatry, 2, e147.

Vargas-Perez, H., Kee, R. T.-A., Walton, C. H., Hansen, D. M., Razavi, R., Clarke, L., ... van der Kooy, D. (2009). Ventral tegmental area bdnf induces an opiate-dependent-like reward state in naive rats. Science, 324(5935), 1732-1734.

Verheij, M. M. M., Karel, P., Cools, A. R., \& Homberg, J. R. (2014). Reduced cocaine-induced serotonin, but not dopamine and noradrenaline, release in rats with a genetic deletion of serotonin transporters [Journal Article]. European Neuropsychopharmacology, 24(11), 1850-1854.

Walsh, R. N., \& Cummins, R. A. (1976). The open-field test: A critical 
review [Journal Article]. Psychological Bulletin, 83(3), 482-504.

Watanabe, M., Nunes, S., Amarante, M., Guembarovski, R., Oda, J., De Lima, K., \& Fungaro, M. (2011). Genetic polymorphism of serotonin transporter 5-HTTLPR: Involvement in smoking behaviour [Journal Article]. Journal of Genetics, 90(1), 179-185.

Weeks, J. R., \& Collins, R. J. (1968). Patterns of intravenous self-injection by morphine-addicted rats. Association for Research in Nervous and Mental Disease, 46, 288-298.

West, M., R. \& Gossop. (1994). Overview: A comparison of withdrawal symptoms from different drug classes [Journal Article]. Addiction, 89(11), 1483-1489.

Williams, R., Peisajovich, S., Miller, O., Magdassi, S., Tawfik, D., \& Griffiths, A. (2006). Amplification of complex gene libraries by emulsion PCR. Nature Methods, 3(7), 545-550.

Willins, D., \& Meltzer, H. (1998). Serotonin 5-HT2C agonists selectively inhibit morphine-induced dopamine efflux in the nucleus accumbens. Brain Research, 781(1-2), 291-299.

Winstanley, C. A., Bachtell, R. K., Theobald, D. E. H., Laali, S., Green, T. A., Kumar, A., ... Nestler, E. J. (2009). Increased impulsivity during withdrawal from cocaine self-administration: Role for delta FosB in the orbitofrontal cortex. Cerebral Cortex, 19(2), 435-444.

Wood, D., \& Emmettoglesby, M. (1988). Substitution and cross-tolerance profiles of anorectic drugs in rats trained to detect the discriminative stimulus properties of cocaine. Psychopharmacology, 95(3), 364-368.

Yang, M., Kavi, V., Wang, W., Wu, Z., \& Hao, W. (2012). The association of 5-HTR2A-1438A/G, COMTVal158Met, MAOA-LPR, DATVNTR and 5-HTTVNTR gene polymorphisms and antisocial personality disorder in male heroin-dependent Chinese subjects. Progress In Neuro-psychopharmacology E Biological Psychiatry, 36(2), 282-289.

Zhu, Z., Zhang, W., Leng, X., Zhang, M., Guan, Z., Lu, J., \& Yang, C. J. (2012). Highly sensitive and quantitative detection of rare pathogens 
through agarose droplet microfluidic emulsion PCR at the single-cell level. Lab on a Chip, 12(20), 3907-3913.

Zimmermann, K., \& Mannhalter, J. W. (1996). Technical aspects of quantitative competitive PCR [Journal Article]. Biotechniques, 21(2), 268279. 